\title{
Efectos entrópicos y de largo alcance en fluidos cargados complejos
}

Tesis que presenta el

$\checkmark$ M. C. Felipe Jiménez Ángeles

\author{
Para obtener el grado de
}

Dcctor en Ciencias

\section{UNIVERSIDAD AIITÓNOMA METROPOLITANA UN'D.TE IZTAPALAPA ${ }^{\top} C B I$}

$\checkmark$ Direc;o: D . Mrreelo Lozada y Cassou $30 \mathrm{~d}$ : septiombre de 2002 
Dedico este trabajo a

Bertha Alicia 


\section{Créditos y agradesimientos}

Agradezco el apoyo económico y facilidades recibidas para la realización de esta tesis a:

- El Consejo Nacional de Ciencia y Tecnología

- La División de Ciencias 'Básicas e Ingeniería y al Departamento de Física de la Universidad Autónoma Metropolitana, unidad Iztapalapa

- El Programa de Ingeniería Molecular del Instituto Mexicano del Petróleo

- Industrias NEGROMEX

Deseo agradecer al Dr. Marcelo Lozada y Cassou el haber dirigido este trabajo, así como sus valiosas enseñanzas.

También agradezco la revisión crítica así como los comentarios constructivos de los miembros del jurado:

- Dr. Fernando del Río Haza (Presidente)

- Dr. Jiang Yu Mei (Secretario)

- Dr. Magdaleno Medina Noyola

- Dr. Mariano López de Haro

Agradezco las colaboración de los Drs. René . Messina. Markus Deserno y Christian Holm; (jueienes calcularon y proporcionaron los datos de simulación molecular presentados en tste tarabajo.

Agratezco a la planta de profesores de la Lniversidad Autónoma Metropolitana, unidad Iztiapalapa. 


\section{Agradecimientos personales}

Agradezco a:

Dios

Agradezco el amor, apoyo y comprensión de mis padres, Irma Ángeles y Felipe Jiménez, así como de mis hermanos Alejandro, Elsa, Luis, Gerardo y Erik y mi abuela Juanita Gutiérrez.

A mi novia Berthà Alicia, agradezco su amor, apoyo y comprensión.

A mis amigos y compañeros. 


\section{Índice General}

iii

Créditos y agradecimientos $\quad$ v

Agradecimientos personales vii

Resumen $\quad$ xi

Introducción general $\quad \dot{x i i i}$

1 Teoría 1

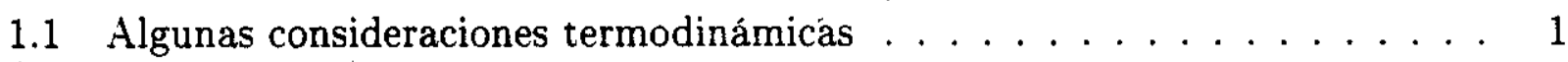

1.2 Funciones de distribución . . . . . . . . . . . . . . . 4

1.2 .1 Ensamble gran canónico . . . . . . . . . . . . . 7

1.3 La ecuación de Ornstein Zernike . . . . . . . . . . . . . . . . 8

1.4 El modelo primitivo . . . . . . . . . . . . . . . . . 9

i.5 Fluidos inhomogéneos: el método directo . . . . . . . . . . 10

1.6 La ecuación de Poisson-Boltzmann . . . . . . . . . . . . . . . 12

1.7 Simulación molecular . . . . . . . . . . . . . . . . . 14

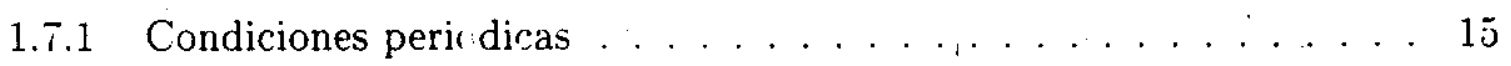

2 Sobrecargado de la molec le é de ADN $\quad 19$

2.1 Introducción . . . . . . . . . . . . . . . . . . . . . . . . 19

2.2 Aplicación de $\mathrm{HNC} / \mathrm{S} \mathrm{A}$ i u u polielectrolito en solución . . . . . . . 22

2.3 Detalles de los calculớs yo dinámca molecular estocástica (DME) . . . . 26

2.3 .1 Potenciales de in ser ac ión . . . . . . . . . . . 26

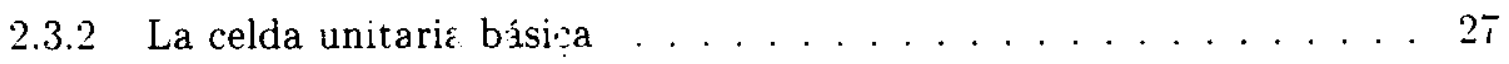

2.3.3 Mapeo de los pa ámetro; a una molécula de ADN . . . . . . 28

2.4 Resultados . . . . . . . . . . . . . . . . . . . . . . . . . 28

2.5 Conclusiones de capítul. $\ldots \ldots \ldots \ldots \ldots \ldots \ldots \ldots$ 
3 Un criı.riu universal para el sobrecargado 39

$3.2 \quad$ La doble capa eléctrica . . . . . . . . . . . . . . . . . . 39

3.2 .1 La ecuación HNC/MSA para una superfi ie plana . . . . . . . . 39

3.2.2 Algunos resultados típicos de HNC/ ISA y Poisson-Boltzmann . . . 43

3.3 Parámetros adimensionales de la ecuación H.NC/.MSA . . . . . . . . 48

3.4 Resultados . . . . . . . . . . . . . . . . . 51

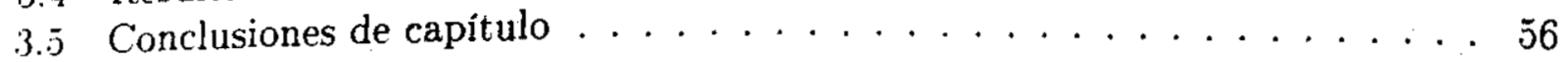

4 Adsorción de macroiones $\quad \mathbf{5 9}$

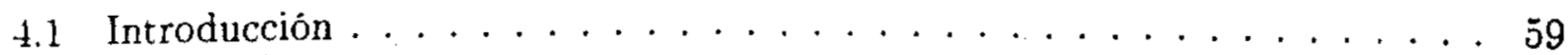

4.2 Modelo . . . . . . . . . . . . . . . . . . . 60

4.3 La Ecuación HNC/MSA . . . . . . . . . . . . . 61

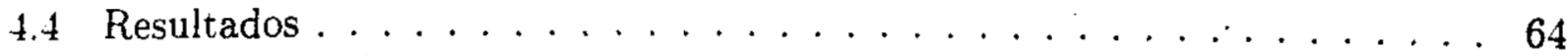

4.5 Conclusiones de capítulo . . . . . . . . . . . . . 69

5 La función de correlación de tres partículas para un electrolito $\quad \mathbf{7 1}$

5.1 Introducción . . . . . . . . . . . . . . 71

5.2 Extensión a tres puntos de las ecuaciones integrales . . . . . . . 72

5.3 La ecuación BGY como una ley de fuerzas . . . . . . . . . . 73

5.4 Aplicación al modelo primitivo restringido $\ldots \ldots \ldots \ldots \ldots$

5.5 Algunos detalles de la dinámica molecular . . . . . . . . . . . . . 79

5.6 Resultados . . . . . . . . . . . . . . . . . . . 80

5.6.1 Electrolito monovalente . . . . . . . . . . . . . . . 80

5.6 .2 Electrolito divalente $\ldots \ldots \ldots \ldots \ldots \ldots$

$5 . \tau$ Conclusiones de capítulo $\ldots \ldots \ldots \ldots \ldots \ldots \ldots$

6 Conclusiones generales y perspectivas $\quad 97$

$\begin{array}{ll}\text { A Diagramas de Mayer } & 101\end{array}$

B Expresiones analiticas para los kérneles 107

$\begin{array}{ll}\text { C La aproximación esférica media } & 109\end{array}$

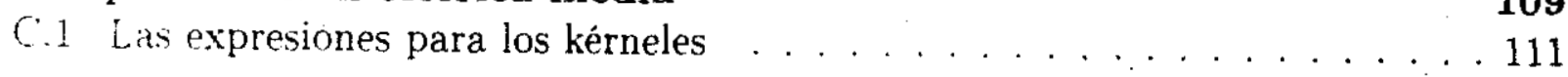

D El método numérico 113

D.1 El método de elemento finito . . . . . . . . . . . . 113

D.2 Las coordenadas de área . . . . . . . . . . . . . . . 116 


\section{Resumen}

En este trabajo estudiamos varios fenómenos que se presentan en los fluidos cargados, homogéneos e inhomogéneos, por medio de diversos métodos de la mecánica estadística de equilibrio: ecuaciones inttegrales, la ecuación de Poisson-Boltzmann y los métodos de simulación molecular, en particular el método de dinámica molecular estocástica. En el primer capítulo presentamos la teoría general de las ecuaciones integrales [desarrolladas sobre la ecuación de Orstein-Zernike (OZ)] así como la teớía de Poisson-Boltzmann. Los métodos de simulación molecular (Monte Carlo (MC) y dinámica molecular estocástica (DME)] son brevemente discutidos en este capitulo asi como la imposición de condiciones periódicas para simular un sistema macroscópico. En este capítulo también discutimos el modelo primitivo, que es con el cual modelamos los sistemas que estudiamos.

En el segundo capítulo se presenta un modelo para una molécula rígida de polielectrolito, inmersa en una solución electrolítica. Por medio de la ecuación integral (HNC/MSA) y DME estudiamos los perfiles de concentración reducida, el potencial electrostático promedio, los perfiles de distribución de carga y el potencial $\zeta$. Como un caso particular, los parámetros del polielectrolito son mapeados a los parámetros de la molécula de ADN. En este estudio se encuentra que ambos, HNC/MSA y DME predicen sobrecargado. Más aun. encontramos un acuerdo cualitativo, y en algunos casos cuantitativo excelente entre HNC/MSA y DME. Las oscilaciones de los perfiles de concentración, la distribución de carga y el potencial electrostático promedio son discutidos en términos del sobrecargado. Los resultados principales en este capítulo son: (i) la predicción por medio de HNC/MSA del comportamiento no monotónico del potencial $\zeta$ el cual es confirmado por DME y (ii) la posibilidad de producir sobrecarga por iones monovalentes la cual es encontrada por ambos, HNC/MSA y DME. Ambos efectos no habian sido reconocidos anteriormente.

En el tercer capítulo, se estudia la sobrecárg: de una superficie plana e infinita por un electrolito tipo modelo primitivo restringide (MPR) simétrico. En primer lugar, mostramos algunos resultados típicos ce ias teor as de Poisson-Boltzmann y HNC/MSA para la doble capa eléctrica. Las apo tacicnes orincipales en este capítulo son: (i) La posibilidad de describir a la doble cape elécrrica ( $a$ ií como al sobrecargado) en términos de tres parámetros adimensionales. Es os parametros están relacionados físicamente con el volumen excluido y las energías de interaccicin :on-ion e ion-superficie. (ii) Presentamos un diagrama de sobrecargado en térmiros de etos tres parámetros, por medio del cual se

\footnotetext{
${ }^{1}$ hypernetted chain / mean spherical approximation
} 
obtiene un criterio $u$ n $i$ vers a l para el umbral de sobrecargado. En este diagrama podemos localizar las condiciones en las que los iones monovalentes producen sobrecargado $y$ tambien aquellas para los iones polivalentes. (iii) Finalmente, en este estudio se presenta al sobrecargado como un estado ordenado que se alcanza al disminuir el número de configuraciones accesibles del sistema, i. e., su entropía.

En el cuarto capítulo analizamos las consecuencias del sobrecargado en la adsorción de macroiones sobre una superficie plana e infinita, cargada uniformemente. En este estudio encontramos útil hacer una distinción entre los términos sobrecargado. iriversión y revertimiento de la carga; los cuales son usados indistintamente en la literatura. A.quí mostramos efectos novedosos de los que podemos destacar los siguientes: (i) La adsorción de macroiones en una superficie (igualmente cargada, con carga opuesta y descargada). (ii) La formación de capas (layering). (iii) Las oscilaciones de los perfiles de concentración de iones puntuales. Nuestra discusión relaciona estos efectos con el sobrecargado, la inversión y el revertimiento de la carga; además de que enfatiza el papel determinante que desempeña la entropía en tales efectos.

En el capítulo 5 presentamos el método denominado extensión a tres puntos (ETP) de las ecuaciones integrales con el cual se incluyen algunas de las correlaciones faltantes en las aproximaciones de la ecuación de $\mathrm{OZ}$, dada la imposibilidad técnica para incluir todas las correlaciones de manera exacta. En este capítulo, la ETP de la ecuación HNC/MSA es aplicada al MPR simétrico. Una de las ventajas que incorpora ETP es la obtención de la función de distribución de tres partículas, $g_{3 \gamma i}^{[3]}\left(\mathbf{r}_{1}, \mathbf{r}_{2}, \mathbf{r}_{3}\right)$. Por otra parte, la fuerza efectiva entre dos partículas del fluido $\mathrm{F}_{\beta \gamma}{ }^{2}$ es también un resultado de ETP. En este capitulo presentamos un estudio mediante ETP y DME de las funciones $g_{\beta \gamma i}^{[3]}\left(\mathbf{r}_{1}, \mathbf{r}_{2}, \mathbf{r}_{3}\right)$ y $\mathrm{F}_{3 \gamma}$ para varios electrolitos. En los regímenes donde las teorías convencionales funcionan correctamente, encontramos que la descripción por medio de ETP es igualmente buena. Más aun, podemos destacar los siguientes resultados: (i) La obtención de la estructura del fluido a nivel de la función de correlación de tres partículas ${ }^{3}$, además de que se obtiene un excelente acuerdo al comparar con los datos de D.IE. (ii) El cambio abrupto en la pendiente de la fuerza entre dos iones igualmente cargados predicho tanto en el régimen diluido como en el régimen concentrado del electrolito. (iii) En el régimen diluido del electrolito. la descripción de ETP mejora la de cualquier otra teoria existente, lo cual es un resultado notable.

\footnotetext{
${ }^{2} \mathrm{~F}_{3 \gamma}$ guarda una relación directa con la función de correlación por pares, $g_{3 \gamma}^{(2)}$, de donde se desprende la comparación con las teorias de líquidos convencionales.

${ }^{3}$ Hasta donde sabemos es la primera vez que esto se hace para el MPR.
} 


\section{Introducción general}

Un coloide es una molécula o un agregado de moléculas, el cual, en al menos una de sus dimensiones mide entre $1 \mathrm{~nm}$ y $1 \mu \mathrm{m}[1,2]$. Esto es, un coloide es una partícula que no es tan pequeña en dimensiones como un átomo, pero no es tan grande como para despreciar las fluctuaciones en densidad y en temperatura del medio en el que se encuentra inmersa. Los polielectrolitos son polímeros portadores de grupos ionizables, los cuales en solución se disocian en macromoléculas cargadas más sus contraiones. En los fluidos cargados, la combinación de las interacciones de largo alcance, así como del efecto colectivo de muchos cuerpos resultan una amplia variedad de fenómenos.

Recientemente, la atracción efectiva entre pares de partículas igualmente cargadas (dispersas en un medio fluido) es uno de los fenómenos que más ha llamado la atención. El efecto se remonta a los trabajos de Norio Ise et al. quienes investigaron la estructura de suspensiones coloidales $[3,4]$. En sus observaciones a lo largo de varios años, estos autores han recolectado una gran variedad de fenómenos aparentemente inconsistentes con la teoría de Derjaguin-Landau-Vewey-Oberveek (DLVO) $[5,6,7,8]$. La teoría DLVO predice que pares de partículas igualmente cargadas, en solución, se repelen. Ise et al., encuentran efectos que van desde la formación anómala de cristales hasta la formación de huecos estables en fluidos coloidales. Estos autores han considerado sus resultados como evidencia de la existencia de atracción de largo alcance entre partículas coloidales igualmente cargadas. El desarrollo de las técnicas experimentales ha permitido la medición de la fuerza efectiva entre pares individuales de partículas. Estas mediciones empiezan a dar coherencia a las observaciones de Norio Ise et al.. De estas mediciones se ha concluido lo siguiente: pares de partículas aisladas se repelen como lo predice la teoría DLVO. Sin embargo, pares de partículas, igualmente cargadas, confinadas por paredes cargadas (de vidrio, por ejemplo) o por concentración de partículas, muestran atracción de largo alcance, lo cual es inconsistente con la teoría DLVO $[9,10,11,12]$. Estas atracciones son favorecidas entre partículas altamente cargadas en soluciones muy diluidas de sal. En estas condiciones se espera que la teoría DLVO falle. En la Fig. 1 se muestra el potencial de interacción medido entre un par de parrículas de poliestireno. Se observa que cuando las partículas no están confinadas se comportan como lo predice la teoría DLVO. Por otra parte, cuando las partículas están confinadas las mediciones muestran una clara atracción entre ellas.

Existe también evidencia de la atraccićn entre segmentos de una misma cadena lineal de polielectrolito, lo cual llega a producir que macromoléculas en solución (que normal- 


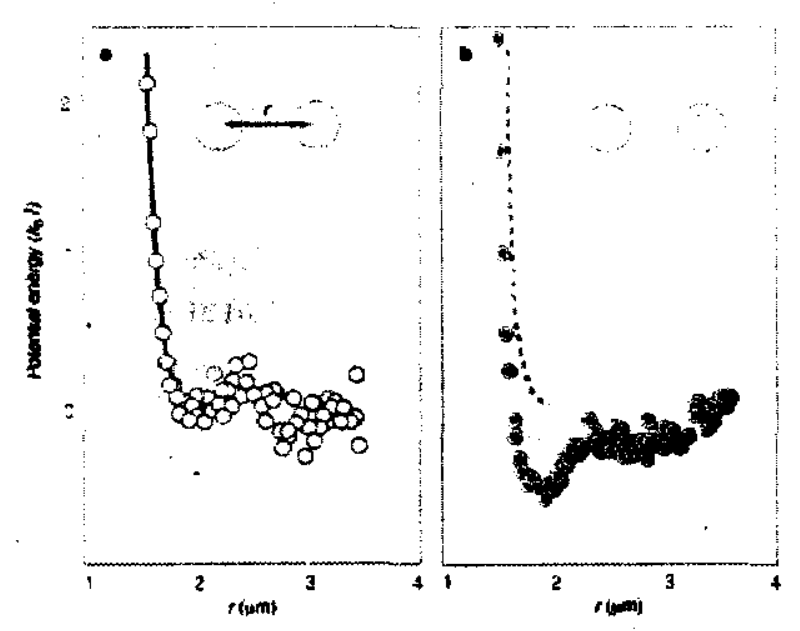

Figura 1: Atracción en confinamiento. El potencial de interacción entre pares de esferas sin confinar es puramente repulsivo (a). Las mediciones de los círculos verdes fueron hechas con esferas de poliestireno de $0.482 \mu \mathrm{m}$ de radio, y muestran acuerde con la teoría DLVO. Sin embargo cuando el mismo par de esferas está confinado for paredes paralelas de vidrio, separadas por una distancia de $\mathbf{3 . 5}$ $\mu \mathrm{m}$, ocurre un mínimo atractivo en el potencial (b). Las partículas se encuentran en solución y adicionalmente están sus contraiones.

mente son rígidas) se colapsen en estructuras compactas cuando se encuentran en presencia de contraiones polivalentes [13, 14]. Estos efectos han volcado a muchos investigadores a estudiar el efecto de la atracción entre pares de partículas igualmente cargadas, en. los marcos teóricos $\{15,16,17,18,19,20,21]$ y de simulación molecular $[22,23,24,25]$.

Un efecto que está íntimamente relacionado con la atracción entre partículas o segmentos de cadena igualmente cargados es, sin duda, el sobrecargado. Dicho efecto, se produce cuando los contraiones de una superficie cargada se acomodan de tal forma que no solamente apantallan la carga de la superficie sino que la sobrecompensan, por lo que dan la apariencia de que la superficie está cargada del signo contrario al que en realidad está. Aunque la doble capa eléctrica ha sido estudiada desde los trabajos clásicos de Gouy y Chapman [26, 27], el sobr cargado fue encontrado hasta los años 80 , en estudios teóricos por ecuaciones integrales $[\because 8,29]$ y de simulación molecular $[30,31]$. Recientemente se ha reconocido la importincia de dicho efecto en la formación de complejos autoensamblados de ADN [32, 33, 34] asi como en experimentos de electroforesis [35]. Esto ha atraído la atención de much us científicos y ha motivado su estudio por medio de teorías semifenomenológicas $[36,37]$ y de ecuaciones integrales $[38,39,40]$, así como de simulación molecular $[41,42,43]$.

La formación de complejos do $A D N$ son biomédicamente importantes debido a que han mostrado ser portadores efecivos de ADN hacia el interior de las células. Cuando se prepara una solucion que contiene $\nprec \mathrm{DN}$ y moléculas hidrocarbonadas catiónicas (lípidos catiónicos) las moléculas de ADN (rarga las negativamente) se asocian espontáneamente con las moléculas lípidas $[32,33,44,45]$. ? a membrana celular normalmente es neutra o se 
encuentra cargada negativamente por lo que tiende a repeler las moléculas de ADN (que son las que nos gustaría introducir dentro de las células por razones de terapia génica). Con ayuda de la formación de complejos de ADN, la barrera electrostática para el ADN sẹpuede disminuir y la entrega genética se facilita. Los complejos de ADN son partículas coloidales que miden alrededor de $0.1 \mu \mathrm{m}$. La carga efectiva de estos coloides depende de la relación de mezclado con las moléculas lípidas. De los experimentos de electroforesis se sabe que si la carga total del ADN excede la carga total cationica, entonces los complejos se encuentran cargados negativamente y coexisten con el exceso de ADN en solución, mientras que en el caso contrario, se encuentran cargados positivamente con exceso de material lípido (sobrecargados). El material catiónico lípido puede sobrecompensar la carga del ADN y aun así los complejos permanecen estables. En la Fig. 2 se muestran varias estructuras internas de los compiejos de ADN con lípidos catiónicos. En lugar de encontrar micelas de lípidos adsorbidas sobre las cadenas de ADN, vemos varias estructuras tipo cristal líquido, reminisentes de los estados laminares y hexagonales formados en lípidos puros o soluciones puras de ADN a alta concentración. En el caso mostrado en la Fig. 2a, los lípidos se encuentran acomodados en un apilamiento laminar de bicapas planas. Las cadenas de ADN son paralelas una con otra en cada capa. La distancia intramolecular entre las moléculas de $\mathrm{ADN}, d_{D N A}$, depende de la relación de mezclado entre las moléculas de ADN y los lípidos catiónicos. En la Fig. 2b, se muestra un ejemplo de una estructura hexagonal invertida, la cual se desempeña mejor que la fase laminar para propósitos de terapia génica. La geometría hexagonal se da en solución de moléculas de ADN más lípidos catiónicos y neutros, a los cuales se les adiciona una especie lípida la cual prefiere curvatura negativa en la interfase con el agua. El radio de la bicapa que se adhiere a las moléculas de ADN es impuesto por la densidad superficial de carga del ADN.

Las interacciones de tipo electrostático en sistemas de muchos cuerpos son importantes no sólo en fluidos complejos y/o sistemas coloidales, sino también en electrolitos simples. En dichos sistemas (por ejemplo, soluciones diluidas de iones polivalentes) existe aún discrepancia en cuanto a su descripción, la cual es difícil debido a que tanto las correlaciones de largo alcance así como las de corto alcance son relevantes. Las ecuaciones integrales convencionales $[46,47,48]$ no los describen completamente bien, debido a que las aproximaciones hechas ignoran ciertas correlaciones. En este trabajo presentamos un método alternativo, denominado extensión a tres puntos de las ecuaciones integrales, con el cual es posible incluír sistemáticamente muchảs de las correlaciones ignoradas en las teorías convencionales de ecuaciones integrales. Así mismo aplicamos este método a un electrolito, con lo cual esperamos una mejor descripción de estos sistemas.

Los fluidos cargados simples y cargados complejos representan un reto con una serie de problemas en donde convergen varias disciplinas científicas como la fisicoqímica y la biofísica asi como la física estadística. El interés en el entendimiento de los problemas mencionados ha crecido rápidamente debido a las aplicaciones tecnológicas que involucran. En este trabajo abordamos varios aspectos de los efectos y fenómenos anteriormente mencionados. Para ello, nos hemos basado en modelos sencillos que toman en cuenta 

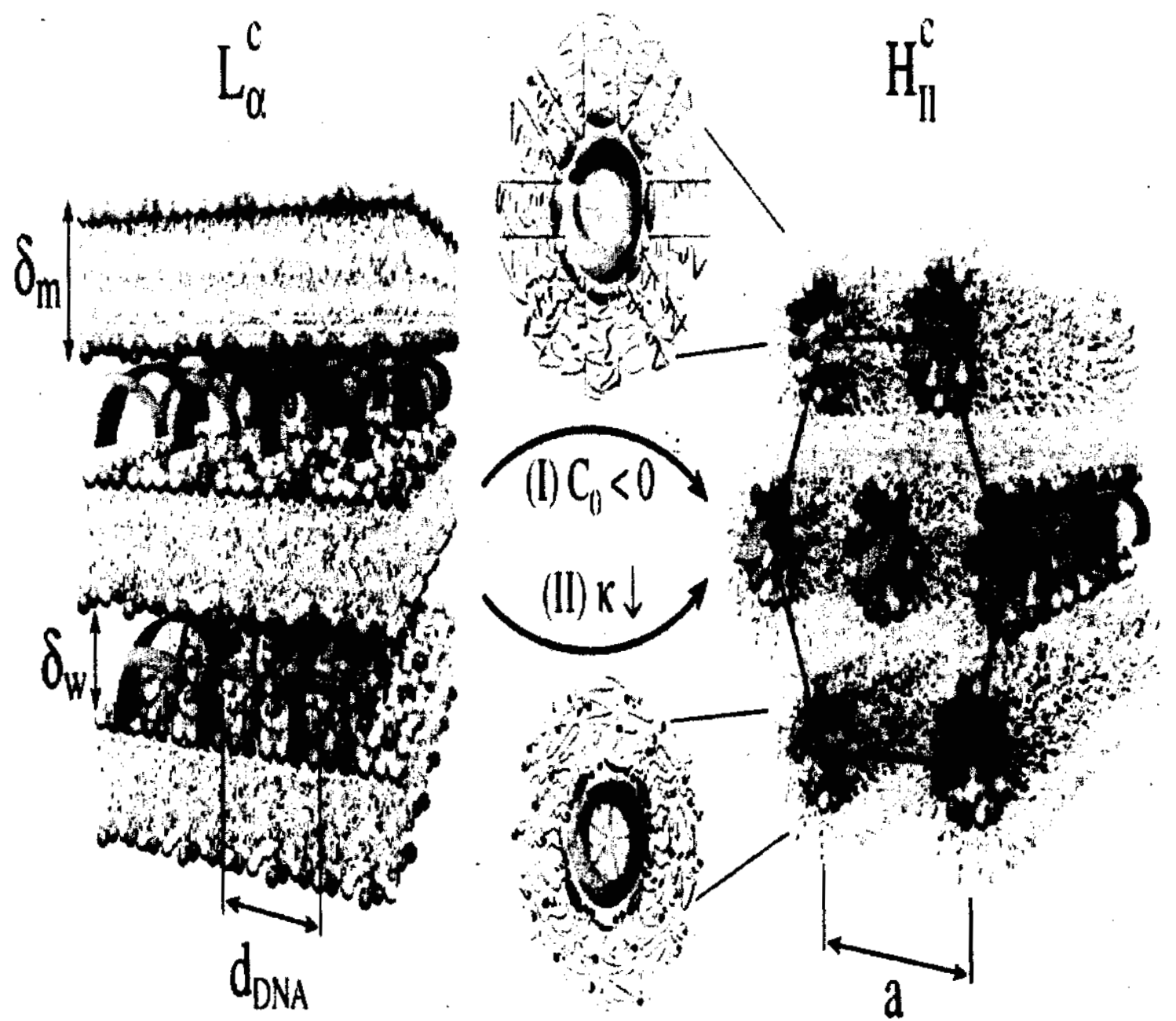

Figura 2: Complejos formados con lípidos catiónicos. (a) Un modelo para el arreglo de los complejos en una fase laminar donde se observa la alternancia entre las bicapas de lípidos y las monocapas de ADN. (b)Modelo para el arreglo local ADN y lípidos catiónicos en una fase hexagonal invertida. Los cilindros consisten de ADN cubierto con una monocapa lípida y arreglados en una fase hexagonal. Las moléculas catiónicas están señaladas con bolas verdes y las blancas denotan moléculas neutras. Las colas hidrocarbonadas se denotan en amarillo. 
algunos de los aspectos más relevantes de los sistemas. En la mayor parte de este trabajo se estudia el sobrecargado en diferentes sistemas. En el capítulo 2, realizamos un estudio de un modelo de una molécula de polielectrolito inmersa en una solución electrolítica. Este estudio se realiza por medio de ecuaciones integrales $y$ los resultados se comparan con datos de simulación molecular. En este estudio observamos que podemos sobrecargar a la molécula por diferentes caminos: incrementando concentración, carga y/o tamaño de las partículas que forman al fluido. Por otro lado, la aparición de una curvatura en la gráfica del potencial $\zeta$ queda confirmada con los datos de simulación moiccular. Erı el capítulo 3, se presenta una parametrización de las ecuaciones de la doble capa aléctrica, encontrándose que depende únicamente de tres parámetros, los cuales son una medide de las energías de interacción y del volumen excluido. De esta forma la sobrecarga es discutida en términos del acoplamiento electrostático y de las relaciones de corto alcance. La importancia de este capítulo radica en que se da un criterio $u n i v$ er $s$ a $l$ para el umbral de la sobreacarga.

En el capítulo 4, se provee de un modelo simple para investigar los procesos involucrados en el autoensamblaje de macroiones en solución. En este capítulo presentamos un modelo de macroiones esféricos en solución más un electrolito, en la vecindad de una superficie plana cargadá. Además presentaremos algunas estructuras autoensambladas predichas por nuestro modelo y analizamos tales estructuras y su relación con el sobrecargado. En el capítulo う presentamos el formalismo de extensión a tres puntos, como ún método para incluir algunas de las correlaciones faltantes en una teoría dada de ecuaciones integralès. En este capítulo aplicacamos ETP a un electrolito. Después de analizar los resultados bajo diversas condiciones, particularmente aquellas que constituyen pruebas fuertes, concluiremos que con ETP se obtiene una mejor descripción del sistema. Al final se incluyen apéndices en donde se presentan de manera detallada los métodos numéricos y los desarrollos algebráicos involucrados a lo largo del trabajo. 


\section{Capítulo 1}

\section{Teoría}

\subsection{Algunas consideraciones termodinámicas}

Dado que el título de este trabajo lo indica, estudiaremos los efectos entrópicos en fluidos cargados complejos, por lo que en esta sección discutiremos nuestro entendimiento de efectos entrópicos. Esta sección es de fundamental importancia debido a que las discusiones aquí presentes son la base de los argumentos con los cuales explicamos nuestros resultados.

En un sistema formado por $\mathrm{N}$ partículas en una caja de volumen $\mathrm{V}$, con una energía total $\mathrm{E}$ y $\Gamma$ estados posibles del sistema; la entropía, $S$, por definición es $[49,50]$

$$
S=k_{B} \log \Gamma
$$

donde $k_{B}$ es la constante de Boltzmann. En equilibrio, $\Gamma$ es conocida si conocemos $\mathrm{N}, \mathrm{V}$ y E. Por lo tanto, $S$ puede pensarse como una función de $E, N$, y $V$

$$
S=S(E, N, V)
$$

Dado que $S$ es una medida del número de configuraciones posibles de un sistema, ésta se incrementará siempre que aumente $E$, manteniendo $N$ yV constantes. Esto es, si aumentamos la energía del sistema habrá más formes de dividirla, lo que implica más configuraciones posibles, por lo tanto, mayor entropía. Si la función $S$ es monotónica. puede ser invertida

$$
E=E(S . N . V)
$$

Si hay cambios en $E$

$$
\mathrm{d} E=\left(\frac{\partial E}{\partial S}\right)_{N . V} \mathrm{~d} S+\left(\frac{\partial E}{\partial \Gamma}\right)_{S . N} \mathrm{dI}+\left(\frac{\partial E}{\partial N}\right)_{S, V} \mathrm{~d} N .
$$

Los coeficientes que multiplican a $\mathrm{d} S, \mathrm{dI}^{-} y \mathrm{~d} . \mathrm{Y}$ en la Ec. (1.1) desempeñan un papel especial en termodinámica. Estos son respectivamente, la temperatura

$$
T=\left(\frac{\partial E}{\partial S}\right)_{x, i}
$$


el negativo de la presión

$$
-P=\left(\frac{\partial E}{\partial V}\right)_{N, S}
$$

y el potencial químico

$$
\mu=\left(\frac{\partial E}{\partial N}\right)_{S, V}
$$

Así, la Ec. (1.1) puede ser escrita como

$$
\mathrm{d} E=T \mathrm{~d} S-P \mathrm{~d} I+\mu \mathrm{d} N .
$$

El enunciado de la primera ley de la termodinám está dado de tal forma que se distinguen en él dos clases de energía (calor y trabajo;. De esta forma, un cambio en $E$ está dado por

$$
\mathrm{d} E=\delta Q+\delta W
$$

donde $Q$ es el calor y $W$ es el trabajo. Los cambios en estas variables son denotados por $\delta Q \circ \delta W$ ya que dependen del tipo de proceso. Sin embargo, los cambios en la energía interna del sistema no dependen del proceso por lo que se denotan como $\mathrm{d} E$. De acuerdo con la Ecs. (1.2) y (1:3), podemos identificar al trabajo como

$$
\delta W=-P \mathrm{~d} V+\mu \mathrm{d} N
$$

donde podemos distinguir dos clases de trabajo: trabajo mecánico $(-P \mathrm{~d} V)$ y trabajo químico $(\mu \mathrm{d} N)$. Por otro lado, en los procesos que se llevan a cabo cuasiestáticamente y de manera reversible podemos identificar.

$$
\delta Q=T \mathrm{~d} S
$$

Un. sistema que incrementa su energia interna. no necesariamente incrementa su entropia (proceso isoetrópico), i. e.. $\mathrm{d} E \neq 0$, mientras que $\mathrm{d} S=0$. Asi por ejemplo, un sistema que incrementa su energía interna cuando se realiza trabajo mecánico sobre él. puede no incrementar su número de estados disponibles (entropia) debido a la compensación por la disminución del volumen. Es decir. el número de estados disponibles que purto haber ganado el sistema al iscrementar su energía interna se ve contrarrestado por lat reducción del volumen. de tal forma que se cumple $\mathrm{d} S=0$.

Si la energía interna de un sistema se incrementa sin realizar trabajo sobre éste. i. e.. $\delta H^{\circ}=0$ : de manera cuasiestática $y$ reversible

$$
\left.\mathrm{d} E\right|_{\mathfrak{W}=0}=\delta Q=T \mathrm{~d} S
$$

Es decir. toda la energía que gana ei sistema se emplea en incrementar el numero de estados posibles del sistema. Lna posibilidad más se da cuando incrementamos la entropía del sistema sin incrementar su energía interna. Teóricamente, esto puede suceder de dos 
formas: Si realizamos trabajo químico sobre el sistema. al agregar partículas a éste y manteniendo $\mathrm{d} E=P \mathrm{~d} V=0$, tenemos entonces

$$
T \mathrm{~d} S+\mu \mathrm{d} N=0 \Rightarrow d S=-\frac{\mu}{T} \mathrm{~d} N .
$$

Esto es, un incremento en el número de partículas corresponde a una disminución de la entropía del sistema, al mantener $E$ y $V$ constantes ${ }^{i}$. Por otro lado, podemos realizar trabajo mecánico manteniendo en este caso $E$ y $N$ constantes, lo cual implica

$$
T \mathrm{~d} S-P \mathrm{~d} V=0 \Rightarrow \mathrm{d} S=\frac{P}{T} \mathrm{~d} I .
$$

En estas condiciones, obtenemos una disminuci" te la entropía al disminuir el volumen del sistema. En ambos casos, i. e., en las ecuacións (1.4) y (1.5) la disminución (aumento) de la entropía tiene que ver con el aumento (disminución) de la concentración, $\rho \equiv \frac{N}{V}$. Algunas propiedades de la entropía pueden verse de las relaciones de Maxwell, por ejemplo en la relación [51]

$$
\left(\frac{\partial S}{\partial P}\right)_{T}=-\left(\frac{\partial V}{\partial T}\right)_{P}
$$

podemos ver que los cambios en la entropía del sistema tienen relación directa con los cambios en volumen del mismo. Vamos a tomar esta ecuación como antecedente de las características de la entropía que discutiremos más adelante. Todos los resultados que hemos discutido hasta aquí son derivados de la termodinámica y por lo tanto, tienen un carácter general. Sin embargo, al considerarse las características microscópicas del sistema estos resultados encuentran diferentes matices.

Como ya mencionamos al principio de esta sección, la entropia es una medida del número de estados posibles de un.sistema, $\Gamma$. Un estado del sistema está definido por los valores que toman las coordenadas y los momentos de las $\mathrm{N}$ partículas que lo forman, $\left\{\mathbf{r}_{1}, \ldots, \mathbf{r}_{N} ; \mathbf{p}_{1}, \ldots, \mathbf{p}_{N}\right\}$. Desde luego que los estados posibles del sistema están determinados por la dinámica de las partículas que lo forman. A su vez, la dinámica de las partículas está determinada por la interacción entre ellas. esto es. depende del tipo de partículas ${ }^{2}$ que forman al sistema.

Cn ejemplo de dos sistemas idénticos, pero con distinto numero de estados accesibles lo constituyen los sistemas como los que se muestran en la Fig. 1.1, en donde se muestran dos sistemas formados por esferas duras. Podemos construirlos de tal forma que ambos tienen la misma energía interna así como el mismo número de partículas, pero las partículas tienen distinto tamaño. El sistema que está formado por partículas más grandes está restringido a un menor número de configuraciones. Por ejemiplo, la configuración en la que la partícula 1 está entre la 2 y la 3 esțá prohibido para el sistema 2 pero no para el sistema 1. Por lo que concluimos que $S_{1}>S_{2}$ siempre que $R_{\mathrm{i}}<R_{2}$. Como vimos

\footnotetext{
${ }^{1}$ Este decremento en $S$ corresponde a $\mu>0$.

${ }^{2}$ El tipo de particula viene dado por el potencial de interacción entre ellas.
} 
anteriormente, el hecho de incrementar la concentración de partículas, $\rho$, trae consigo un decremento de la entropía también. En sistemas formados por partículas duras la entropía está relacionado con la fracción de volumen accesible ${ }^{3}$ en el sistema, por lo que podemos decir que a una mayor fracción de volumen accesible mayor entropia del sistema.
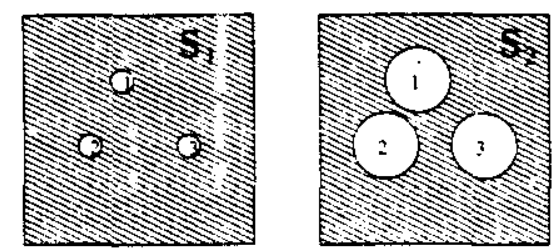

Figura 1.1: Dos sistemas idénticos formados con igual número de partículas $\mathrm{N}$ y con la misma energía interna $\mathrm{E}$. El sistema 1 esta formado por partículas más pequeñas, i. e., $R_{1}<R_{2}$. La fracción de volumen accesible está representada por la región sombreada.

Cuando las partículas que forman al sistema interaccionan vía un potencial repulsivo, las configuraciones en las que las partículas están más cerca entre sí, están más restringidas a medida que el potencial repulsivo es de mayor intensidad y/o de mavor alcance. Esto es, para dos sistemas con igual número de partículas $N$, volumen $V$ y energía interna $E$; la entropía es menor para el sistema que está formado por partículas que interaccionan vía un potencial repulsivo de mayor intensidad $\mathrm{y} / \mathrm{o}$ de mayor alcance. De esta forma, para alcanzar las configuraciones en las que las partículas pueden estar más cercanas entre sí, la energía interna del sistema debe incrementarse. De hecho, podemos establecer que: $a$ mayor intensidad y/o alcance del potencial (repulsivo en este caso), la entropia del sistema disminuye, por lo que el caso discutido en la Fig. 1.1 es sólo un caso particular.

\subsection{Funciones de distribución}

Los estados microscópicos (caracterizado por el conjunto de posiciones y momentos de las partículas $\left.\left\{\mathbf{r}_{1} \ldots \mathbf{r}_{N}, \mathbf{p}_{1} \ldots \mathbf{p}_{N}\right\}\right)$. de un sistema determinan, en gran parte, sus propiedades termodinámicas. Sin embargo. las cantidades macroscópicas medidas en el laboratorio son ślamente cantidades promedio de los estados microscópicos del sistema. Con los métodos de la mecánica estadística de equilibrio podemos calcular la estructura ${ }^{4}$ de un fluido, que a su vez. nos sirve para calcular sus propiedades termodinámicas. A continuación vamos a. resumir algunos resultados de la mecánica estadística de equilibrio(MEE), útiles para él estudio de los fluidos $[52,53,54]$.

En el estudio de fluidos por medio de la .IEE. el concepto de función de distribución es de utilidad. Esta función mide la probabilidad de encontrar a las partículas del fluido

\footnotetext{
"La fracción de volumen accesible es la razón entre el volumen no ocupado y el volumen total del sistema.

${ }^{\dagger}$ Este concepto será aclarado más adelante.
} 
en cierta configuración. En un sistema de partículas que interactúan vía un potencial dado, a $\mathrm{T}, \mathrm{V}$ y $\mathrm{N}$ constantes (en el ensamble canónico), la probabilidad de encontrar a la partícula 1 en un elemento de volumen $\mathrm{d} \mathbf{r}_{1}$ en $\mathbf{r}_{1}$, a la partícula 2 en un elemento de volumen $\mathrm{dr}_{2}$ en $\mathbf{r}_{2}$, etc., está dada por

$$
P\left(\mathbf{r}_{1} \ldots \mathbf{r}_{N}\right) d \mathbf{r}_{1} \ldots d \mathbf{r}_{N}=\frac{\exp \left\{-\beta U_{N}\left(\mathbf{r}_{1} \ldots \mathbf{r}_{N}\right)\right\}}{Z_{N}} \mathrm{~d} \mathbf{r}_{1} \ldots \mathrm{d} \mathbf{r}_{N}
$$

donde $Z(N, V, T)=\int \ldots \int \exp \left\{-\beta U_{N}\left(\mathbf{r}_{1} \ldots \mathbf{r}_{N}\right)\right\} \mathrm{d} \mathbf{r}_{1} \ldots \mathrm{d} \mathbf{r}_{N}$ es la función de particiún con-figuracional en el ensamble canónico y $U_{N}\left(\mathbf{r}^{N}\right)$ es la energía configuracional de las $N$ partículas. La densidad de probabilidad de encontrar a la partícula 1 en $\mathbf{r}_{1}, \ldots$, y a la partícula $\mathrm{n}$ en $\mathbf{r}_{n}$, independientemente de donde se encuentran las restantes, se encuentra integrando sobre las coordenadas de las partículas, desde $n+1$ hasta $N$

$$
P^{(n)}\left(\mathbf{r}_{1} \ldots \mathbf{r}_{n}\right)=\frac{\int \ldots \int \exp \left\{-\beta U_{N}\left(\mathbf{r}_{1} \ldots \mathbf{r}_{N}\right)\right\} \mathrm{d} \mathbf{r}_{n+1} \ldots \mathrm{d} \mathbf{r}_{N}}{Z_{N}}
$$

Por otro lado, la densidad de probabilidad de encontrar a cualquier partícula en $\mathbf{r}_{1}, \ldots, \mathbf{y}$ a cualquier partícula en $\mathbf{r}_{n}$, independientemente del resto de las partículas está dada por

$$
\rho^{(n)}\left(\mathbf{r}_{1} \ldots \mathbf{r}_{n}\right)=\frac{N !}{(N-n) !} P^{(n)}\left(\mathbf{r}_{1} \ldots \mathbf{r}_{n}\right)
$$

La función $g^{(n)}\left(\boldsymbol{r}^{(n)}\right)$, definida como

$$
g^{(n)}\left(\mathbf{r}_{1} \ldots \mathbf{r}_{n}\right)=\frac{\rho^{(n)}\left(\mathbf{r}_{1} \ldots \mathbf{r}_{N}\right)}{\rho^{n}}
$$

es conocida como la función de correlación entre $n$ partículas y determina el grado de independencia, i. e., la correlación entre las partículas. Si las partículas de un sistema fueran independientes $g^{(n)}\left(\mathbf{r}^{(n)}\right)$ sería simplemente uno. Usando las Ecs. (1.6), (1.7) y (1.8). $g^{(n)}\left(\mathbf{r}^{(n)}\right)$ se escribe explícitamente como

$$
\begin{aligned}
g^{(n)}\left(\mathbf{r}_{1} \ldots \mathbf{r}_{n}\right) & =\frac{I^{n} V !}{N^{n}(N-n) !} \frac{\int \ldots \int \exp \left\{-3 L_{N}\left(\mathbf{r}_{1} \ldots \mathbf{r}_{N}\right)\right\} \mathrm{d} \mathbf{r}_{n+1} \ldots \mathrm{d} \mathbf{r}_{N}}{Z_{N}} \\
& =V^{n}\left(1+O\left(N^{-1}\right)\right) \frac{\int \ldots \int \exp \left\{-3 L_{N}\left(\mathbf{r}_{1} \ldots \mathbf{r}_{N}\right)\right\} \mathrm{d} \mathbf{r}_{n+1} \ldots \mathrm{d} \mathbf{r}_{N}}{Z_{N}}
\end{aligned}
$$

La función de correlación entre pares, $g^{(2)}\left(\mathbf{r}_{1}, \mathbf{r}_{2}\right)$, mide la densidad de probabilidad de encontrar a una partícula en $\mathbf{r}_{1}$ y otra en $\mathbf{r}_{2}$, por lo que esta función proporciona la estructura promedio del sistema. Para el caso de partículas que interactúan vía potenciales esféricos, $g^{(2)}\left(\mathbf{r}_{1}, \mathbf{r}_{2}\right)$ depende sólo de la distancia entre las partículas, $r=\left|\mathbf{r}_{2}-\mathbf{r}_{1}\right|$, por lo 
que suele denotarse como $g(r)$. La cantidad $\rho g(r)$ mide la concentración local de partículas medida desde una prtícula central, por lo que satistace

$$
\int_{0}^{\infty} \rho g(r) 4 \pi r^{2} \mathrm{~d} r=N-1 \approx N
$$

Suponiendo que las partículas del sistema interacciona i vía un potencial central aditivo por pares, $u(r)$, se pueden escribir las cantidades termo linámicas en función de $g(r)$. Por ejemplo, la energía interna se escribe de la siguiente m.nera

$$
E=\frac{3}{2} N k_{B} T+\frac{N}{2} \int_{0}^{\infty} \rho u(r) g(r) 4 \pi r^{2} \mathrm{~d} r
$$

De la misma forma puede escribirse una ecuación análoga para la presión

$$
p=\rho k T-\frac{\rho^{2}}{6} \int_{0}^{\infty} r \frac{d u(r)}{d r} g(r) 4 \pi r^{2} \mathrm{~d} r
$$

La función $g(r)$ es de particular importancia desde el punto de vista experimental ya que el factor de estructura, $S(k)$, se puede medir mediante experimentos de dispersión de luz o dispersión de partículas. La relación entre $S(k)$ y $g(r)$ está dada a través de la transformada de Fourier, i. e.,

$$
S(k)=\int g(r) e^{-i \mathbf{k} \cdot \mathbf{r}} \mathrm{d} \mathbf{r}
$$

De esta forma. conociendo $S(k)$ podemos determinar $g(r)$ mediante la transformada inversa de Fourier.

Una forma conveniente de escribir $g^{(n)}\left(\mathbf{r}^{(n)}\right)$ es como

$$
g^{(n)}\left(\mathbf{r}_{1} \ldots \mathbf{r}_{n}\right)=\exp \left\{-3 u^{(n)}\left(\mathbf{r}_{1} \ldots \mathbf{r}_{n}\right)\right\}
$$

donde la cantidad $u^{(n)}\left(\mathbf{r}^{(n)}\right)$ queda definida al sustituir ésta en la definición de $g^{(n)}$, Ec. (1.9). tomar el logaritmo de ambos lados. y tom r el gradiente respecto a las coordenadis de una de las $n$ particulas, lo cual nos da como resultado

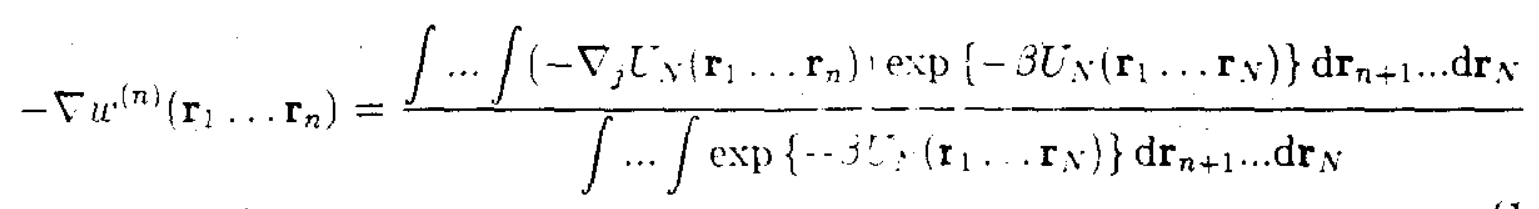

Como sabemos $-\nabla_{j} U_{N}\left(\mathbf{r}_{1} \ldots \mathbf{r}_{N}\right)$ es la fuerze que actúa sobre la partícula $j$ (con $1 \leq$

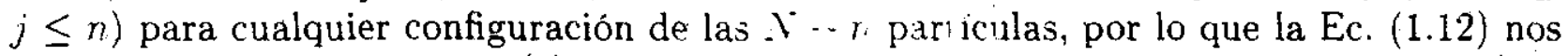
representa li fuerza media $f_{j}^{(n)}$ sobre la particuia $j$. promediada sobre la configuración de todas las $n+1 \ldots N$ partículas. Es decir $u^{(n)}$ es el potencial de la fuerza promedio actuando sobre la partícula $j$. 


\subsubsection{Ensamble gran canónico}

La teoria de funciones de distribución es rás poderosa si se desarrolla usando el ensamble gran canónico. A continuación presentamo; un resumen de las propiedades de las funciones de distribución de un sisterna clásico, abi rto y de una sola especie. Dado que vamos a generalizar las ideas de la sección anterior ‘ sistemas abiertos, las funciones de distribución [Ecs. (1.7) y (1.8)] se denotan como $\rho_{N}^{(n)}$ y $g_{N}^{(n)}$. Entonces, la probabilidad de encontrar $n$ partículas en los elementos de volumen $\mathrm{d} \mathbf{r}_{1}, \ldots, \mathrm{d} \mathbf{r}_{n}$ en $\mathbf{r}_{1}, \ldots, \mathbf{r}_{n}$, independientemente de $N$, es

$$
\rho^{(n)}=\sum_{N \geq n} \rho_{N}^{(n)} P_{N}
$$

donde $P_{N}$ es la probabilidad de que un sistema contenga a $N$ particulas, es decir

$$
P_{N}=\frac{e^{N \mu / k_{B} T} Q(N, V, T)}{\Xi(\mu, V, T)} \doteq \frac{z^{N} Z_{N}}{N ! \Xi}
$$

donde

$$
\begin{aligned}
Z_{N} & =Z(N, V ; T)=\int \ldots \int \exp \left\{-\beta U_{N}\left(\mathbf{r}^{N}\right)\right\} \mathrm{d} \mathbf{r}_{1} \ldots \mathrm{d} \mathbf{r}_{N} \\
z & =\frac{e^{\mu / k_{B} T}}{\Lambda^{3}} \\
\Xi & =\sum_{N \geq 0} \frac{z^{N} Z_{N}}{N !}
\end{aligned}
$$

con $A=\left(\frac{2 \pi m k_{B} T}{h^{2}}\right)^{-1 / 2}$. De esta forma, usando las Ecs. (1.7) y (1.8) en la Ec. (1.13), se obtiene

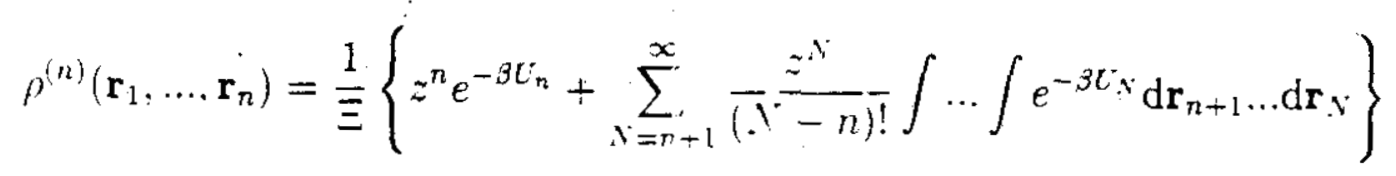

donde $L_{j}$ denota la energía config rácirni l le un sistema de $j$ partículas. La definición dada en la Ec. (1.14) es el equivalerite de la $E:(1 . T)$ para un sistema abierto. De la misma manera, la definición de la función cie cor re aciór entre $n$ particulas para un sistema abierto es igual a la de la Ec. (1.9). Por lo que és:a se escribe simplemente como

$$
g^{(n)}\left(\mathbf{r}_{1}, \ldots, \mathbf{r}_{n}\right)=\frac{V^{n}}{\bar{N}^{n}} \equiv\left\{z^{n} e^{-3 t}+\sum_{\Lambda=n+1}^{\infty} \frac{z^{N}}{\left(\lambda-\frac{z^{\prime}}{n}\right.} \int \ldots \int e^{-\beta U_{N}} \mathrm{~d} \mathbf{r}_{n+1} \ldots \mathrm{d} \mathbf{r}_{N}\right\}
$$


Las funciones de distribución en los ensambles canónico y gran canónico son las mismas para sistemas macroscópicos [55], por lo que las Ecs. (1.10) y (1.11) se siguen cumpliendo, a menos que se involucren las fluctuaciones ${ }^{5}$.

Dada la definición de $g(r)$ en el ensamble gran canónico [Ec. (1.15)] se puede hacer un desarrollo en serie de ésta en potencias de $\rho$. Los primeros términos de este desarrollo son

$$
\begin{aligned}
g\left(\mathbf{r}_{1}, \mathbf{r}_{2}\right) & =e^{-\beta u\left(\mathbf{r}_{1}, \mathbf{r}_{2}\right)}\left\{1+\rho \int f_{13} f_{23} \mathrm{~d} \mathbf{r}_{3}+\rho^{2} \int\left(f_{13} f_{34} f_{42}+2 f_{13} f_{34} f_{42} f_{32}+\right.\right. \\
& \left.\left.+\frac{1}{2} f_{13} f_{41} f_{32} f_{42}+\frac{1}{2} f_{13} f_{41} f_{34} f_{32} f_{41}\right) \mathrm{~d} \mathbf{r}_{3} \mathrm{~d} \mathbf{r}_{4}+\rho^{3} \int\left(f_{13} f_{34} f_{45} f_{52}+\ldots\right) \mathrm{d} \mathbf{r}_{3} \mathrm{~d} \mathbf{r}_{4} \mathrm{~d} \mathbf{r}_{5}+\ldots\right\}
\end{aligned}
$$

donde

$$
f_{i j} \equiv e^{-\beta u\left(\mathbf{r}_{i}, \mathbf{r}_{j}\right)}-1
$$

es conocida como la función de Mayer. El $n$-ésimo coeficiente es una suma de integrales de productos de la función de Mayer. Una integral de productos de las funciones de Mayer tiene una representación gráfica conocida como un diagrama de Mayer [52, 53, 56]. En el apéndice $\mathrm{A}$ daremos un breve resumen sobre la construcción y clasificación de los diagramas de Mayer a los cuales haremos referencia posteriormente.

\subsection{La ecuación de Ornstein Zernike}

La función de correlación total se define como $h\left(\mathbf{r}_{1}, \mathbf{r}_{2}\right) \equiv g\left(\mathbf{r}_{1}, \mathbf{r}_{2}\right)-1$, para un fluido de una sola especie. En 1914 Ornstein [5T] y Zernike [58] proponen dividir $h\left(\mathbf{r}_{1}, \mathbf{r}_{2}\right)$ en dos partes, una parte directa y una parte indirecta. La parte directa, dada por la función $c\left(\mathbf{r}_{1}, \mathbf{r}_{2}\right)$, llamada la función de correlación directa. La parte indirecta es la influencia propagada directamente de la partícula 1 a la 3 , la cual actúa sobre la partícula 2. directamente e indirectamente a través de otras partículas. Este efecto es pesado por la concentración y promediado sobre todas las posiciones de la partícula 3. Con esta descomposición de $h\left(\mathbf{r}_{1}, \mathbf{r}_{2}\right)$, se escribe la ecuación de Ornstein-Zernike.

$$
h\left(\mathbf{r}_{21}\right)=c\left(\mathbf{r}_{21}\right)+\rho \int h\left(\mathbf{r}_{23}\right) c\left(\dot{\mathbf{r}}_{13}\right) \mathrm{d} \mathbf{r}_{3} .
$$

donde hemos supuesto que el potencial de interacción entre las particulas depende únicamencte de su posición relativa, $\mathbf{r}_{21}=\mathbf{r}_{2}-\mathbf{r}_{1}$. por lo que $h\left(\mathbf{r}_{1}, \mathbf{r}_{2}\right)=h\left(\mathbf{r}_{21}\right)$.

De la Ec. (1.12). la función $g\left(\mathbf{r}_{21}\right)$ está relacionada con el potencial de la fuerza media entre dos partículas, $u\left(\mathbf{r}_{21}\right)$, por

$$
g\left(\mathbf{r}_{21}\right)=\exp \left\{-3 x^{\prime}\left(\mathbf{r}_{21}\right)\right\}
$$

En un fluido multicomponenie, la función de correlación total $\left(h_{i j}\left(\mathbf{r}_{21}\right) \equiv g_{i j}\left(\boldsymbol{r}_{21}\right)-1\right)$ entre dos particulas de las especies $i$ y $j$ en $\mathbf{r}_{1}: \mathbf{r}_{2}$. respectivamente, están relacionadas

\footnotetext{
${ }^{5}$ Ver ref. [55]
} 
con la función de correlación directa $c_{i j}\left(\mathbf{r}_{21}\right)$ por la tcuación de Ornstein-Zernike multicomponente, la cual, para un sistema de $k$ componentes está dada por

$$
h_{i j}\left(\mathbf{r}_{21}\right)=c_{i j}\left(\mathbf{r}_{21}\right)+\sum_{m=1}^{k} \rho_{m} \int h_{i m}\left(\mathbf{r}_{23}\right) c_{m j}\left(\mathbf{r}_{13}\right) \mathrm{d} \mathbf{r}_{3},
$$

donde $\rho_{m}$ es la concentración de la especie $m$ y $\mathbf{r}_{21} \equiv \mathbf{r}_{1}-\mathbf{r}_{2}$ es la posición relativa entre las partículas 1 y 2. La ecuación de Ornstein-Zernike define la función de correlación directa. En esta ecuación tenemos dos funciones y ambas son desconocidas. Para poder resolver esta ecuación tenemos que emplear una teoría que nos de una relación entre la función de correlación directa y la función de correlación total. Se han propuesto varias aproximaciones para la función de correlación directa fundamentadas en métodos de la teoría de líquidos. Entre estas aproximaciones tenemos las siguientes [53, 54]

$$
\begin{gathered}
c_{i j}\left(\mathbf{r}_{21}\right)=-\dot{\beta} u_{i j}\left(\mathbf{r}_{21}\right)+h_{i j}\left(\mathbf{r}_{21}\right)-\ln g_{i j}\left(\mathbf{r}_{21}\right), \\
c_{i j}\left(\mathbf{r}_{21}\right)=-\beta u_{i j}\left(\mathbf{r}_{21}\right), \\
c_{i j}\left(\mathbf{r}_{21}\right)=f_{i j}\left(\mathbf{r}_{21}\right) g_{i j}\left(\mathbf{r}_{21}\right) \exp \left\{\beta u_{i j}\left(\mathbf{r}_{21}\right)\right\}
\end{gathered}
$$

Las Ecs. (1.22), (1.23) y (1.24) se conocen como la aproximación de cadena hipertejida (HNC), la aproximación esférica media (MSA), y la aproximación de Percus-Yevick (PY), respectivamente. Siendo $u_{i j}\left(\mathbf{r}_{21}\right)$ el potencial de interacción directa entre un par de partículas de las especies $i$ y $j$ y $f_{i j}\left(\mathbf{r}_{21}\right) \equiv \exp \left\{-\beta u_{i j}\left(\mathbf{r}_{21}\right)\right\}-1$, es la función de Mayer ${ }^{6}$. No es posible calcular $g\left(\mathbf{r}_{21}\right)$ de forma exacta ya que todas las teorías para calcularla ignoran varios tipos de diagramas. Por ejemplo, en la teoría HNC los diagramas puente son ignorados completamente, mientras que en la teoría de Percus-Yevick los diagramas puente y los diagramas producto no son considerados (ver apéndice A). La representación gráfica del los diagramas de primero y segundo orden están dados en la Fig. 1.3. En la columna de la izquierda se encuentra la representación exacta de la función de distribución mientras que en el centro y el lado derecho está la representación diagramáticas de las teorías HNC y PY, respectivamente.

\subsection{El modelo primitivo}

El modelo más simple que contiene los principales rasgos de un electrolito es el modelo primitivo (MP) [59]. Este modelo consiste en aproximar el solvente por un medio continuo en el cual los iones se encuentran inmersus ${ }^{7}$. La interacción entre los iones se lleva a cabo

\footnotetext{
${ }^{6}$ Esta definición difiere de la dada en la Ec. (1.18) donde $i$ y $j$ denotan posiciones, mientras que en este caso denotan especies.

${ }^{7}$ Este medio continuo tiene una constante dieléctrica $\varepsilon$, diferente de la constante dieléctrica del vacio.
} 


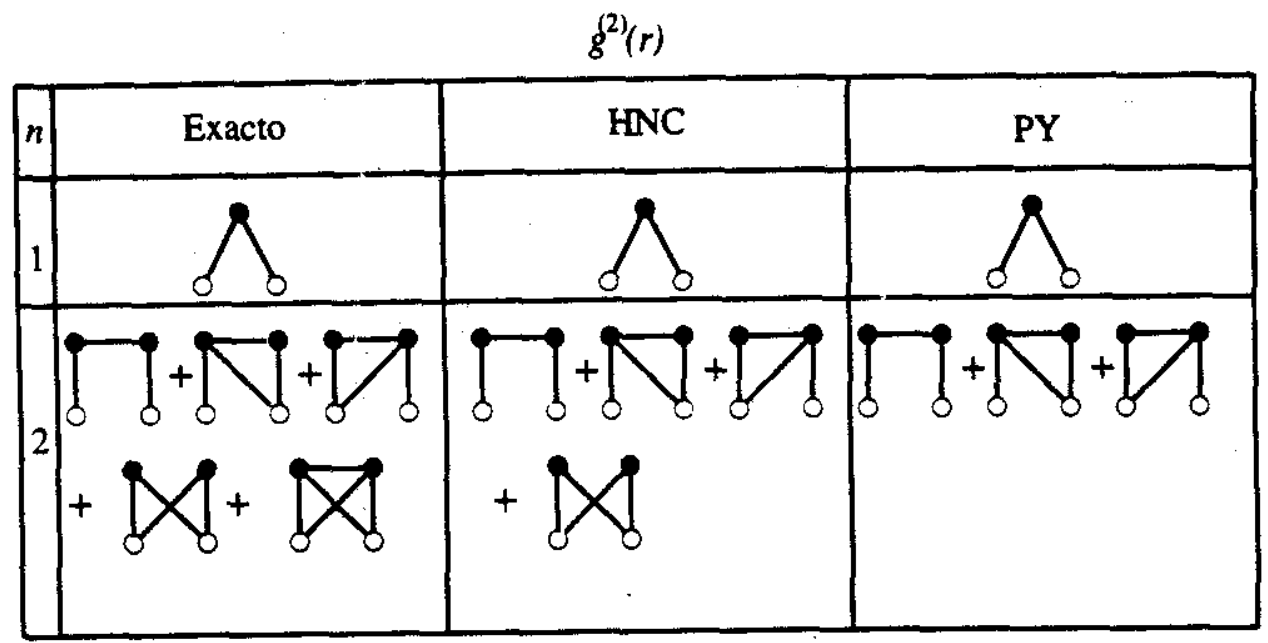

Figura 1.2: Diagramas de Mayer de primero y segundo orden de la función de correlación por pares en su desarrollo exacto (izquierda), la aproximación HNC (centro) y la aproximación de Percus-Yevick (derecha).

vía un potencial, el cual, para dos iones con carga $q_{i}$ y $q_{j}$ separados por una distancia $r$, está dado por

$$
u_{i j}(r)=\left\{\begin{array}{ccc}
\infty & \text { si } & r<\frac{a_{i}+a_{j}}{2} \\
\frac{q_{i} q_{j}}{\varepsilon r} & \text { si } & r \geq \frac{a_{i}+a_{j}}{2}
\end{array}\right.
$$

Donde $q_{i}=z_{i} e$ y $a_{i}$ son la carga y el diámetro del ion de la especie $i$, respectivamente; $z_{i}$ es el número de valencia y $e$ es la carga protónica. Es decir, estamos tratando a los iones como esferas duras cargadas. En el modelo primitivo restringido (MPR) todas las especies que forman al electrolito tienen el mismo tamaño. Para alcanzar el equilibrio termodinámico el fluido debe satisfacer la condición de electroneutralidad la cual se escribe de la siguiente forma

$$
\sum_{i=1}^{2} z_{i} \rho_{i}=0,
$$

donde $\rho_{i}$ es la concentración de bulto de la especie $i$.

\subsection{Fluidos inhomogéneos: el método directo}

Un fluido influenciado por un campo externo o un fluido que se encuentra en la vecindad de la superficie de una partícula coloidal. se acomodan de manera inhomogénea. En esta sección vamos a extender los métodos de la sección 1.3 al estudio de fluidos inhomogéneos. 
Las ecuaciones integrales ya han sido aplicados por Henderson et al. al estudio de interfases [60,61]. Además de la propuesta de Kjellander et al. [62] donde también obtiene la función de distribución inhomogénea. Sin embargo, estos métodos solamente pueden ser empleados para fluidos en contacto con superficies planas.

Un método más general y con el cual pueden derivarse teorías para fluidos inhomogéneos fue propuesto por Lozada-Cassou [56,63], este método es conocido como el Método Directo (MD). Este método es más general que el método de Henderson, ya que puede emplearse para cualquier geometría. Por otro lado, con este método puede obtenerse como un caso particular el caso de Henderson. El método directo esta basado en el hecho fundamental de la equivalencia entre partículas y campos. Es decir, el hecho de que las partículas y campos se definen a través de su interacción con otras particulas o campos. Ya que en las teorías presentadas en la sección 1.3 no existe restricción en cuanto al tipo de partícula, el número de especies en el fluido y la concentración de cada especie; una fuente de campo externo puede ser considerada como una especie más del fluido, la cual se encuentra a dilución infinita. De esta forma, si llamamos $\alpha$ a la especie que representa a la fuente de campo externo, partiendo de la Ec. (1.21) para $n+1$ especies y tomando el caso límite de $\rho_{n+1}=\rho_{\alpha} \rightarrow 0$, la función de correlación entre la especie $\alpha$ y la especie $j$ está dada por:

$$
h_{\alpha j}\left(\mathbf{r}_{21}\right)=c_{\alpha j}\left(\mathbf{r}_{21}\right)+\sum_{m=1}^{n} \rho_{m} \int h_{\alpha m}\left(\mathbf{r}_{23}\right) c_{m j}\left(\mathbf{r}_{13}\right) d \mathbf{r}_{3},
$$

nótese que la suma no considera la especie $n+1$. Análogamente a la Ec. (1.20), la relación entre la función de distribución, $g_{\alpha i}\left(\mathbf{r}_{21}\right)$, y el potencial de la fuerza promedio entre la partícula $\alpha$ y una partícula de la especie $i$ en $\mathbf{r}_{21}$ viene dada por

$$
g_{\alpha i}\left(\mathbf{r}_{21}\right)=\exp \left\{-\beta w_{\alpha i}\left(\mathbf{r}_{21}\right)\right\} .
$$

Para poder resolver la Ec. (1.27) es necesario conocer $c_{m j}\left(\mathbf{r}_{13}\right)$ y $c_{\alpha j}\left(\mathbf{r}_{21}\right)$. La primera $\left[c_{m j}\left(\mathbf{r}_{13}\right)\right]$ se obtiene de la solución de la ecuación de Orstein-Zernike para un fluido homogéneo de $n$ especies, como la Ec. (1.21). La segunda función $\left[c_{\alpha j}\left(\mathbf{r}_{21}\right)\right]$ puede aproximarse por cualquiera de las cerraduras PY, MSA ó HNC, las cuales tienen la siguiente forma funcional

$$
\begin{gathered}
c_{\alpha i}\left(\mathbf{r}_{21}\right)=f_{\alpha i}\left(\mathbf{r}_{21}\right) g_{\alpha i}\left(\mathbf{r}_{21}\right) \exp \left\{3 u_{\alpha i}\left(\mathbf{r}_{21}\right)\right\}, \\
c_{\alpha i}\left(\mathbf{r}_{21}\right)=-3 u_{\alpha i}\left(\mathbf{r}_{21}\right), \\
c_{\alpha i}\left(\mathbf{r}_{21}\right)=h_{\alpha i}\left(\mathbf{r}_{21}\right)-\ln g_{\alpha i}\left(\mathbf{r}_{: 1}\right)-\beta u_{\alpha i}\left(\mathbf{r}_{21}\right),
\end{gathered}
$$

Pueden construirse distintas teorias de líquidos dependiendo de las aproximaciones que se usen para $c_{\alpha i}$ y $c_{i j}$. Aunque formalmente no existe justificación para mezclar diferentes aproximaciones, se ha encontrado que las fallas de algunas teorías son parcialmente 
enmendadas cuando se usa una teoría hibrida. In ejemplo ampliamente usado lo es la mezcla entre la teorias HNC y MSA, la cual ha mostrado que funciona relativamente bien para sistemas cargados bajo una ampl a gê ma de condiciones y que además, subsana parcialmente los defectos que ambas tería;, H VC y MSA, presentan por separado. La notación que se usa para denotar dichas teorias es la siguiente:

Siglas que denotan a $c_{\alpha i} / \mathrm{Si}$ jlas que denotan a $c_{i j}$.

Ejemplo de las teorias que podemos emplear son: $\mathrm{HNC}_{/} \mathrm{HNC}, \mathrm{PY} / \mathrm{HNC}, \mathrm{MSA} / \mathrm{HAC}$, etc. Este método puede emplearse en general para cualquier tipo de fluido, sin cmbargo, ha sido aplicado principalmente para el caso de fluidos cargados inhomogéneos, las cuales han resultado exitosas al compararse con los métodos de simulación molecular $[40,64,65]$.

\subsection{La ecuación de Poissơn-Boltzmann}

Cuando un electrolito se encuentra influenciado por un campo eléctrico el cual es producido por una superficie o por una partícula coloidal con carga, los contraiones (particulas cargadas del signo opuesto) son atraídos hacia la superficie, mientras que los coiones (partículas cargadas del mismo signo) tienden a ser repelidos. Debido a que el sistema se encuentra a $T>0$, i. e., las partículas del fluido tienen energía cinética, los contraiones no condensan totalmente sobre la superficie. Por otro lado, los coiones no son totalmente repelidos de la superficie debido a que no pueden disponer de todo el espacio accesible. Los contraiones y coiones se arreglan de tal forma que eventualmente el campo eléctrico es cancelado a cierta distancia de la superficie. Esta estructura es conocida como la doble capa eléctrica(DCE).

El tratamiento más simple para fluidos cargados se lleva mediante la ecuación clásica de Poisson-Boltzmann. En esta teoría, además de que solamente se consideran las interacciones de tipo electrostático. se emplea una aproximación sobre el potencial de la fuerza promedio de dos partículas, $w^{(2)}\left(\mathbf{r}_{12}\right)[$ Ec. (1.28)]. Consideremos una macropartícula inmersa en un electrolito en equilibrio. a la cual llamaremos la partícula $\alpha$. De la electrostática sabemos que el potencial eléctricu depende de la distribución de carga como lo indica la ecuación de Poisson $[66]$

$$
\nabla^{2} \varphi(\mathbf{r})=-\frac{4}{\vdots} p_{e l}(\mathbf{r})
$$

donde $\rho_{e l}(\mathbf{r})$ es la densidad local de carsá en $\mathbf{r}$ ia rual está definida por

$$
\left.\rho_{e l}, \mathbf{r}\right)=\sum_{i=1}^{n^{*}} \sum_{i} e e_{i}(\mathbf{r})
$$

siendo $\rho_{i}(\mathbf{r})$ la concentración local de la especie $i$. Si calculamos el promedio de ensamble de ambos lados de la Ec. (1.32) tene'nos cor:10 resuliario

$$
\nabla^{2}\langle\varphi(\mathbf{r})\rangle=-\frac{4 \pi}{\Xi}\left\langle\rho_{e^{\prime}}(\mathbf{l})\right\rangle=-\frac{2 \pi}{\varepsilon} \sum_{=1}^{n} z_{i} e\left\langle\rho_{i}(\mathbf{r})\right\rangle
$$


donde los promedios de ensamble se indican con las cantidades entre corchetes. El promedio de la concentración local de la especie $i$ está relacionada con la función de distribución por

$$
\left\langle\rho_{i}(\mathbf{r})\right\rangle=\rho_{i} g_{\alpha i}(\mathbf{r}),
$$

donde $g_{\alpha i}(\mathbf{r})$, es la función de distribución de la especie $i$ alrededor de la especie $\alpha$ y $\rho_{i}$ es la concentración de bulto de la especie $i$. Usando la Ec. (1.35), la Ec. (1.34) se puede escribir como

$$
\nabla^{2}\langle\varphi(\mathbf{r})\rangle=-\frac{4 \pi}{\varepsilon} \sum_{i=1}^{n} z_{i} e \rho_{i} g_{\alpha i}(\mathbf{r}) .
$$

De la Ec. (1.28) sabemos que la función de distribución de la especie $i$ alrededor de $\alpha$ está relacionado con el potencial de la fuerza promedio sobre la partícula $i$ en $r$. La teoría de Debye-Hückel supone que $[53,67]$

$$
w_{\alpha i}(\mathbf{r})=z_{i} e\langle\varphi(\mathbf{r})\rangle
$$

por lo que

$$
g_{\alpha i}=\exp \left\{z_{i} e\langle\varphi(\mathbf{r})\rangle\right\} .
$$

Si denotamos $\langle\varphi(\mathbf{r})\rangle=\dot{\psi}(\mathbf{r})$, la Ec. (1.36) se escribe como

$$
\nabla^{2} \psi(\mathbf{r})=-\frac{4 \pi}{\varepsilon} \sum_{i=1}^{n} z_{i} e \rho_{i} \exp \left\{-\beta z_{i} e \psi(\mathbf{r})\right\}
$$

Esta ecuación diferencial es conocida como ecuación de Poisson-Boltzmann. De la electrostática sabemos que lạ solución de la ecuación de Poisson, Ec. (1.32), puede escribirse como

$$
\psi(\mathbf{r})=\psi_{\alpha}(\mathbf{r})+\frac{1}{\varepsilon} \int \frac{\rho_{e l}\left(\mathbf{r}_{1}\right) d \mathbf{r}_{1}}{\left|\mathbf{r}-\mathbf{r}_{1}\right|},
$$

donde $\psi_{\alpha}(\mathbf{r})$ es la contribución al potencial debida a la partícula $\alpha$, y el segundo término es la contribución al potencial de siaa a la distribución de carga del fluido. Haciendo uso de la Ecs. (1.3T) y (1.40), obtenetncs

$$
\begin{aligned}
g_{\alpha i}(\mathbf{r}) & =\exp \left\{-\beta_{2 i}^{e l}(\mathbf{r})-\frac{z_{i} e 3}{\Sigma} \int \frac{\rho_{e l}\left(\mathbf{r}_{1}\right) d \mathbf{r}_{1}}{\left|\mathbf{r}-\mathbf{r}_{1}\right|}\right\}, \\
& =\exp \left\{-\beta_{i}^{e l} e_{\alpha i}^{e l}(\mathbf{r})-\frac{z_{i} \epsilon^{2} 3}{\Xi} \sum_{j=1}^{n} z_{j} \rho_{j} \int \frac{g_{\alpha j}\left(\mathbf{r}_{1}\right) d \mathbf{r}_{1}}{\left|\mathbf{r}-\mathbf{r}_{1}\right|}\right\},
\end{aligned}
$$

en donde $u_{\alpha i}^{e l}(\mathbf{r})=z_{i} e \psi_{\alpha}(\mathbf{r})$. L' cua' es otra forma de escribir la ecuación de PoissonBoltzmann, o mejor dicho, este es la versión integral de la ecuación de Poisson-Boltzmann. La ecuación de Poisson-Boltzrna!n ha sido la base de numerosos trabajos, p. ej., los trabajos de Gouy [26] y Chapman [27], en: donde estudiaron una solución electrolítica en 
presencia de un campo externo producido por una superficie plana cargada uniformemente. Las aproximaciones de las que hace uso la ecuación de Poisson-Boltzmann fueron examinadas rigurosamente por Kirkwood [68], quien demostró que la aproximación consiste en despreciar los términos de volumen excluido y de fluctuaciones. Aunque el análisis de Kirkwood fue hecho para electrolitos en bulto, éste puede ser extendido de manera directa para la doble capa eléctrica. La comparación entre los resultados de la ecuación de Poisson-Boltzmann con los de simulación molecular, muestra que la teoría falla cuando el volumen excluido no es despreciable o cuando las interacciones electrostáticas; so.n nauy fuertes [69].

\subsection{Simulación molecular}

Una técnica importante de carácter casi experimental es la simulación por computadora. Existen dos métodos que han sido ampliamente utilizados: El método de Monte Carlo (MC) [70] inventado por Metropolis et al. y el método de dinámica molecular (DM) introducido por Alder y Wainwright [71]. Posteriormente, la técnica de dinámica molecular estocástica (DME) fue propuesta por Ermak $[i 2]^{8}$. Este tipo de cálculos proveen lo que puede considerarse como un resultado exacto, dada la fuerza de interacción molecular. La utilidad de estos métodos reside en el hecho de que un modelo que contiene un número de partículas relativamente pequeño, típicamente algunos cientos, en la mavoria de los casos es suficiente para simular el comportamiento de un sistema macroscópico. En la técnica de dinámica molecular, las ecuaciones clásicas de movimiento de un sistema de partículas que interactúan se resuelven y las propiedades de equilibrio son determinadas por promedios temporales tomados sobre un intervalo de tiempo suficientemente largo (típicamente $\sim 10^{-10}-10^{-11}$ s en tiempo real). El método de Monte Carlo involucra la generación de una serie de configuraciones de las partículas de un modelo en una forma que se asegure que las configuraciones estén distribuidas en el espacio fase de acuerdo con algunas densidades de probabilidad prescritas. El valor medio de cualquier propiedad configuracional determinada a partir de un número suficientemente grande de configuraciones $\left(\sim 10^{6}\right)$ provee una estimación del promedio de ensamble de la dicha cantidad. siendo el carácter de promedio de ensamble dependiente del procedimiento de muestreo que sea usado. La mayor ventaja del método de dinámica molecular es que permite el estudio de procesos dependientes del tiempo. Sin embargo. el metodo de $\mathrm{MC}$ ofrece rentajas sobre D.M para estudiar procesos independientes del tiempo.

Los resultados de simulación que mostraremos en este trabajo fueron obtenidos mediante DIE. por lo que a continuación discutiremos brevemente esta técnica. La técnica de DME consiste en resolver las ecuacion 's de morimiento de las partículas del sistema que se está modelando ${ }^{9}$. En DME se planttan las ecuaciones de inovimiento de un conjunto de

\footnotetext{
${ }^{8}$ Debe resaltarse que el cálculo de las propiedades estáticas de un fluido (por ejemplo la función de distribución radial, $g(r)$ ) es independiente dt la técnica de simulación usada (73).

${ }^{9}$ Cabe aclarar que los datos de DNE que mostraremos aqui fueron calculados por René Messina y
} 
partículas acopladas a un baño térmico que actúa vía una fuerza estocástica $\mathbf{W}(t)$, cuyo valor medio es cero. De esta forma la ecuación de movimiento para la $i$-ésima partícula está dada por

$$
m \frac{d^{2} \mathbf{r}_{i}}{d t^{2}}=-\nabla_{i} U-m \Gamma \frac{d \mathbf{r}_{i}}{d t}+\mathbf{W}(t)
$$

en donde $m$ es la masa de la partícula, $\Gamma$ es el coeficiente de fricción y $U$ es el potencial. Al suponer aditividad por pares, $U$ se escribe como

$$
U=\sum_{i<j}^{N} u_{i j}+\sum_{i=1}^{N} v_{i},
$$

donde $u_{i j}$ es el potencial de interacción entre cualquier par de iones y $v_{i}$ es el potencial de interacción entre la $i$-ésima partícula y el campo externo. En nuestros modelos, $u_{i j}$ tiene dos contribuciones: (i) las interacciones por volumen excluido y (ii) las interacciones de tipo Coulomb. Dada la imposibilidad técnica para utilizar esferas duras en la simulación, la interacción por volumen excluido entre cualquier par de partículas es modelada vía un potencial tipo Lennard-Jones repulsivo, el cual está dado por

$$
u_{i j}^{L J}(r)= \begin{cases}4 \epsilon\left[\left(\frac{a}{r}\right)^{12}-\left(\frac{a}{r}\right)^{6}\right]+\epsilon & \text { si } r<2^{1 / 6} a \\ 0 & \text { si } r \geq 2^{1 / 6} a .\end{cases}
$$

La interacción electrostática entre cualquier par de iones $i$ y $j$ está dada por

$$
u_{i j}^{\text {coul }}(r)=\frac{q_{i} q_{j}}{\varepsilon r}=k_{B} T \ell_{B} \frac{z_{i} z_{j}}{r}
$$

donde $\ell_{B} \equiv \frac{e^{2}}{\varepsilon k_{\mathrm{B}} T}$. Para llevar a cabo la comparación entre el resultado de HNC/MSA y los de simulación molecular usamos $T=298 \mathrm{~K}, a=4.25 \AA \mathrm{y} \varepsilon=78.5$, que dan como resultado $\ell_{B}=7.14 \AA$. Si el electrolito está confinado en una caja cúbica de longitud $\mathrm{L}$. La concentración de sal está dada por $\frac{N}{L^{3}}$, donde $Y$ es el número de iones positivos (o negativos).

\subsubsection{Condiciones periódicas}

Al estudiar un sistema microscópico por medio de las técnicas de simulación molecular, se encueatran varios inconvenientes. Lno de estos inconvenientes es el de que no se puede tener un sistema con un número de partículas tan grande como un sistema macroscópico real. Por el contrario, debido a las dificultades de almacenamiento y al tiempo de cálculo, se suelen usar sistemas con menos de $10^{4}$ partículas, que desde luego está muy lejos

Markus Deserno, siguiendo los métodos descritos en las referencias $[23,24.25,74,75,76]$ 
del número de partículas en un sistema macroscópico. Si estamos interesados en una microgota o un microcristal, las fuerzas de cohesión pueden ser suficientes para mantener a las $\mathrm{N}$ moléculas en agregación. Otra forma de modelar el sistema, puede ser la de confinarlo por un potencial que simule una caja y de esta forma prevenir que las moléculas se separen. Sin embargo, estos arreglos pueden no ser suficientes para estudiar sistemas en bulto, debido a que el efecto de las paredes es significantemente diferente que el efecto que le produce, a una muestra de fluido, el fluido que la rodea en el bulto.

El problema de los efectos de superficie puede ser superado con un artificio propuesto por Born y von Karman [77, 78]. En esta técnica, se forman réplicas de la muestra de fluido que se está simulando, la cual normalmente es cúbica, acomodándolas en una red periódica infinita. En el curso de la simulación, cuando una molécula se mueve en la caja original, su imagen en cualquiera de las cajas vecinas se mueve de la misma forma. De esta manera, cuando una molécula sale de la caja central, una de sus imágenes entra inmediatamente por la cara opuesta. De esta forma, no, hay superficies que rodeen a la muestra eliminándose así sus efectos. Una versión bidimensional de un sistema periódico es mostrado en la Fig. (1.3).

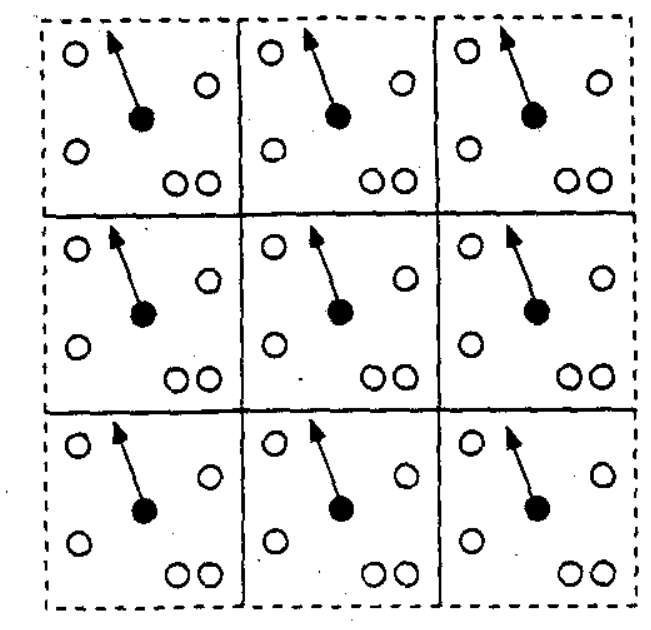

Figura 1.3: Ejemplo de un sistema periódico bidimensional. Las moléculas pueden entrar y salir a través de cualquiera de las cuatro fronteras. En un sistema tridimensional hay 6 caras por donde las moléculas pueden entrar y salir.

Cuando las moléculas que forman al sistema interaccionan vía un potencial de corto alclance (por ejemplo como el de la Ec. (1.44)], el cálculo de la energía configuracional de las partículas dentro de la celda principal. i. e.. Ec. (1.43), considera únicamente las interacciones con los vecinos de las celdas contiguas. En el caso de particulas que interaccionan via un potencial de largo alcance (como el potencial de Coulomb de la Ec. (1.45) | esta aproximación no puede mantenerse. En el caso de potenciales de largo alcance debe considerarse la interacción de una partícula de la celda principal con las partículas de las celdas contiguas más todas aquellas que se mantienen dentro del alcance 
del potencial. Esta tarea se vuelve más complicada a medida que el potencial se vuelve de mayor alcance. Las sumas de Ewald pueden emplearse para llevar a cabo la tarea de calcular la energía de interacción en un sistema periódico, de manera eficiente [79]. Vamos a mostrar como funciona la suma de Ewald en el cálculo de la energía electrostática de interacción de los iones de la celda principal. Pera ello vamos a considerar un sistema de $N$ partículas con cargas $q_{i}$ en las posiciones $\mathbf{r}_{i}$ en una celda cúbica de longitua $L \mathbf{y}$ volumen $V_{b}=L^{3}$. En un sistema periódico, la energía electrostática total de la cája está dada por

$$
U=\frac{1}{2} \sum_{i, j=1}^{N} \sum_{\mathbf{n} \in \mathbf{Z}^{3}}^{1} \frac{q_{j} q_{i}}{\mathbf{r}_{i j}+\mathbf{n} L}
$$

La suma sobre todas las $\mathbf{n}$ toma en cuenta las imagenes periódicas de las cargas $\mathbf{y}$ la prima indica que en el caso de $i=j$ el término $\mathbf{n}=0$ debe omitirse y se ha definido $\mathbf{r}_{i j} \equiv \mathbf{r}_{i}-\mathbf{r}_{j}$. Del tratamiento de Ewald resulta la fórmula de Ewald para la energia electrostática de la caja:

$$
U=U^{(r)}+U^{(k)}+U^{(s)}+U^{(d)}
$$

donde la contribución en el espacio real $U^{(r)}$, la contribución del espacio recíproco $U^{(k)}$, la energía de autointeracción $U^{(s)}$ y la contribución dipolar $U^{(d)}$ están dádas por

$$
\begin{aligned}
U^{(r)} & =\frac{1}{2} \sum_{i, j=1}^{N} \sum_{\mathbf{n} \in \mathbf{Z}^{3}} q_{j} q_{i} \frac{\operatorname{erfc}\left(\alpha\left|\mathbf{r}_{i j}+\mathbf{m} L\right|\right)}{\left|\mathbf{r}_{i j}+\mathbf{m} L\right|}, \\
U^{(k)} & =\frac{1}{2 L^{3}} \sum_{\mathbf{k} \neq 0} \frac{4 \pi}{k^{2}} e^{-k^{2} / 4 \alpha^{2}}|\rho(\mathbf{k})|^{2}, \\
U^{(s)} & =-\frac{\alpha}{\sqrt{\pi}} \sum_{i} q_{i}^{2}, \\
U^{(d)} & =\frac{2 \pi}{\left(1+2 \epsilon^{\prime}\right) L^{3}}\left(\sum_{i} q_{i} \mathbf{r}_{i}\right)^{2},
\end{aligned}
$$

donde la funcion de error está definida por $\operatorname{erfc}(r)=2 \pi^{-1 / 2} \int_{r}^{\infty} \mathrm{d} t \exp \left\{t^{2}\right\}$ y la transformada de Fourier de la densidad de carga $\rho(\mathbf{k})$ está dada por

$$
\rho(\mathbf{k})=\int_{V_{b}^{\prime}} \mathrm{d}^{3} r \rho(\mathbf{r}) e^{-i \mathbf{k} \cdot \mathbf{r}}=\sum_{j=1}^{Y} q_{j} e^{-i \mathbf{k} \cdot \mathbf{r}_{j}}
$$

La longitud inversa $\alpha$, conocida como parámetro de Ewald, asigna los pesos relativos a las contribuciones del espacio real $y$ el espacio recíproco, pero el resultado final es independiente de $\alpha$. Los vectores $\mathbf{k}$ forman el conjunto discreto $\{2 \pi \mathbf{n} / L: \mathbf{n} \in \mathbf{Z}\}$. De esta forma, pueden emplearse técnicas eficientes para el el cálculo de la energía de la celda principal. La eficiencia en dicho cálculo dependerá, básicamente, de las técnicas empleadas en el cálculo de la transformada de Fourier. 


\section{Capítulo 2}

\section{Sobrecargado de la molécula de ADN}

\subsection{Introducción}

En la ref. [76] la distribución iónica ha sido estudiada por medio de la función de distribución de carga, $P(r)$, la cual es calculada mediante dinámica molecular estocástica. En el presente capítulo realizamos un estudio del sobrecargado por medio de ecuaciones integrales $[29,80]$ así como un estudio más detallado de simulación molecular. Presentamos además cálculos para $P(r)$, los perfiles de concentración $g_{i}(r)$; el potencial electrostático promedio $\psi(r)$, y el potencial $\zeta$. Así mismo presentamos una comparación entre los resultados de la teoría con los obtenidos de dinámica molecular.

Debido a su inobjetable estatus como molécula de la vida, la molécula del ácido desoxirribonucléico ( $\mathrm{ADN}$ ) ha sido sujeta un número abrumador de pruebas estructurales, cinéticas $y$ termodinámicas, más que cualquier otra molécula. Con una carga efectiva de una carga elemental (negativa) por cada $0.17 \mathrm{~nm}$ a lo largo de su longitud, la molécula de $A D N$, tiene casi tanta carga como un polímero lineal. La estructura de doble hélice de la molécula de $A D N$ es bien conocida, así como el hecho de que la información genética es llevada en su secuencia de pares de bases. Sin embargo, en este trabajo, su estructura de doble hélice es significante solamente por el hecho de que le da gran rigidez a la molécula: La molécula de ADN mantiene esencialmente su misma dirección sobre su relativamente alta longitud de persistencia. 'Jtra propiedad estructural importante de la molécula de AD. es el hecho de que la molécula es altamente cargada en condiciones fisiológicas estándar. eso significa, condiciones de $\mathrm{pH}=7.4$. El diámetro de una molécula de $\mathrm{ADN}$ es de aproximadamente $2 \mathrm{~nm}$, mientras que sus longitudes típicas varían desde algunas micras para virus simples hasta centímetros en humanos [81].

En busca del entendimiento de las propiedades del ADN se han encontrado comportamientos inesperados en la conformación de dicha molécula [13]: En condiciones fisiológicas (0.1 molar de solución de $\mathrm{NaCl})$ una molécula de $\mathrm{ADN}$ toma la forma de una madeja enrollada en forma desordenada con un radio de giro de varias micras; si dos segmentos cualesquiera de la molécula se arercan a una distancia menor a $1 \mathrm{~nm}$ éstos se repelen fuertemente. Sin embargo, bajo condiciones diferentes, por ejemplo en una solución muy 


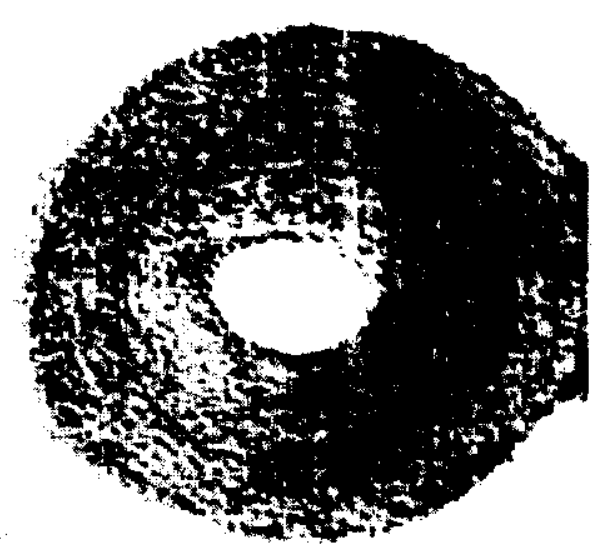

Figura 2.1: Condensación de la molécula de ADN

diluida con una concentración pequeña de cationes polivalentes, la misma molécula de ADN se condensa en un rollo de forma toroidal altamente empaquetado. En la Fig. 2.1 se muestra la configuración toroidal de la molécula de ADN. Su radio promedio es de unos $\mathbf{5 0}$ $\mathrm{nm}$ y la distancia promedio entre dos segmentos contiguos paralelos es ligeramente mavor que su diámetro. Un toro esencialmente con las mismas características y dimensiones se forma con otro tipo de contraiones, otras secuencias genéticas así como con piezas de $\mathrm{ADN}$ mucho más pequeñas. Un toro mucho más grande se forma cuando el $\mathrm{ADN}$ es expulsado desde un virus hacia una solución que contenga iones polivalentes. Claramente, los contraiones polivalentes median la atracción efectiva entre las moléculas de ADN cargadas negativamente, esto en una.contradicción aparente del hecho fundamental de que los objetos con carga del mismo signo se repelen.

Este hecho vuelve interesante a este sistema desde un punto de vista fundamental así como desde un punto de vista tecnológico. Las interacciones entre los segmentos mismos de la molécula așí como con la memurana celular (que en parte se deben al acoplamiento entre la distribución iónica y lạ cinformación de la cadena están aún muy lejos de ser completamente entendidas. "En in primera aproximación al estudio de un polielectrolito en solución, se puede fijar la crintornación de la cadena. de esta forma, se puede uno enfocar en la descripción detaliada de la distribución iónica. Usualmente los polielectrolitos se encuentran estirados debido a li repulsión electrostática de sus grupos cargados. Más aun, muchos polielectro itris ieneiı una rigidez intrínseca, por lo que un modelo de varilla rígida para estas molécul is puede: ser una primera elección. El problema restante de una varilla rígida cargada innıersa en una solución es mucho más fácil de tratar, pero aún lejos de ser completo.

En el tratamiento del problema, uná aproximacion connúnmente usada supone que la investigación de un pequeño subvolumen ( l cual cortiene solamente una varilla cargada, 
Figura 2.2: Representación esquemática del efecto de sobrecargado. Las bolas sombreadas y blancas representan a los contraiones y coiones, respectivamente. En la parte inferior está indicada la dirección y magnitud que debería tener el campo eléctrico

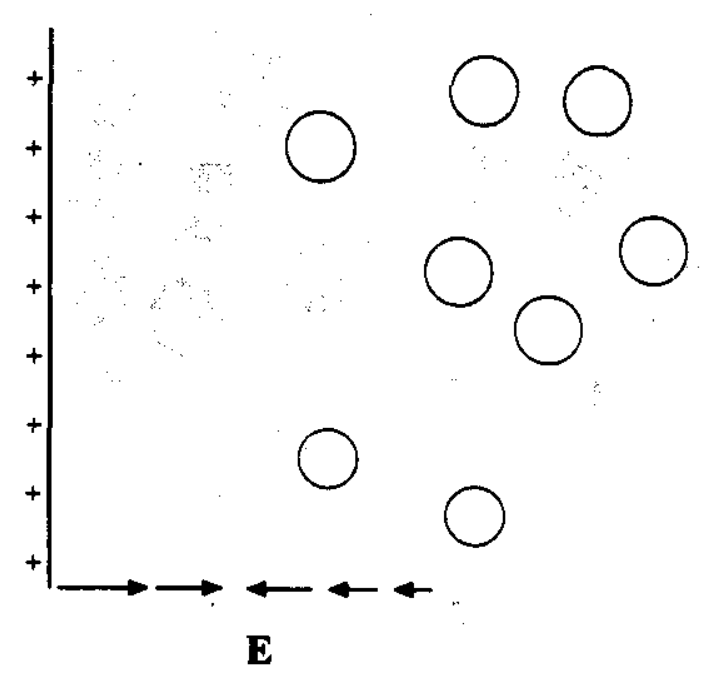

sus contraiones y el electrolito) es suficiente para describir mucha de la física. La principal justificación para el uso de esta aproximación es que el subvolumen tiene carga neta igual a cero por lo que los contraiones apantallan eficientemente los multipolos de orden superior y por lo tanto, la interacción entre dos subvolúmenes es prácticamente nula. Esta aproximación es conocida como el modelo de la celda cilíndrica y éste provee el marco para los cálculos de simulación molecular aquí presentados.

El sobrecargado ocurre cuando la distribución del fluido es tal que para una distancia dada a la superficie, los contraiones no solamente cancelan la carga de la superficie, sino que además se arreglan de tal forma que la sobrecompensan. Esto implica una inversión en la dirección del campo eléctrico. En la Fig. 2.2 se encuentra representado el tipo de arreglo que debe existir en el fluido para que exista sobrecargado. Cerca de la superficie los contraiones sobrecompensan la carga de la superficie, sin embargo, el sistema en total permanece neutro.

La doble capa eléctrica obtenida por medio de las ecuaciones integrales predicen sobrecargado $[29,80]$. Anteriormente el sobrecargado ha sido observado en cálculos de simulación por Monte Carlo (MC) para geometría plana [82], cilíndrica [83] y esférica $[84,85]$. Además, hace algunos años, Gonzalez-Tovar et al. [29] predijeron que para una carga dada del macroión y concentración del electrolito, el sobrecargado produciría un revertimiento de la movilidad electroforética: Este revertimiento de la movilidad electroforética ha sido confirmado experimentalment $|36.87,88,89|$. Al considerar este efecto en una nueva teoría para la electroforesis, se ha encontrado un acuerdo [35] cualitativo excelente con resultados experimentales $[87,91]$.

La teoria de Poisson-Boltzmenti muestra sus limitaciones en las situaciones donde las correlaciones de corto alcanc: ent:e los iones se vuelven importantes [69, 91], además de que no predice las fuerzas at ractivas observadas experimentalmente en soluciones de ADN $[13,92,93]$, a pesar de qlie sto ha sido predicho incorrectamente por algunos autores [21]. El sobrecargado ha sido pr dicho en estudios hechos mediante simulación molecular $[94,95,96]$ y ecuaciones integral s $[16.97,98]$. En este capitulo presentamos estudios para 
una varilla cargada, inmersa en una solución salina, bajo situaciones que no son descritas correctamente por la teoría de Poisson-Boltzmann, es decir, exploramos la posibilidad de sobrecargado de una sola varilla.

\subsection{Aplicación de $\mathrm{HNC} / \mathrm{MSA}$ a un polielectrolito en solución}

Una ecuación particularmente exitosa para fluidos inhomogéneos cargados, es a denominada $\mathrm{HNC} / \mathrm{MSA}[84,99,100,101]$. La ecuación HNC/MSA para un modelo de un polielectrolito cargado en solución, se puede deducir con los métodos descritos en el capítulo 1. Dicha ecuación ya ha sido obtenida anteriorn e $[29,80]$ considerando lo siguiente: El polielectrolito es modelado como una varilla tigida infinitamente larga, de radio $r_{0}$, con una densidad lineal de carga $\lambda$. La varillả está inmersa en un electrolito tipo modelo primitivo restringido de dos componentes. El solvente es considerado como un medio dieléctrico continuo de una constante dieléctrica $\varepsilon$. Por simplicidad suponemos que la varilla y el solvente tienen la misma constante dieléctrica, para evitar complicaciones con las cargas imagen (ver Fig. 2.3). La condición de electroneutralidad del fluido viene dada

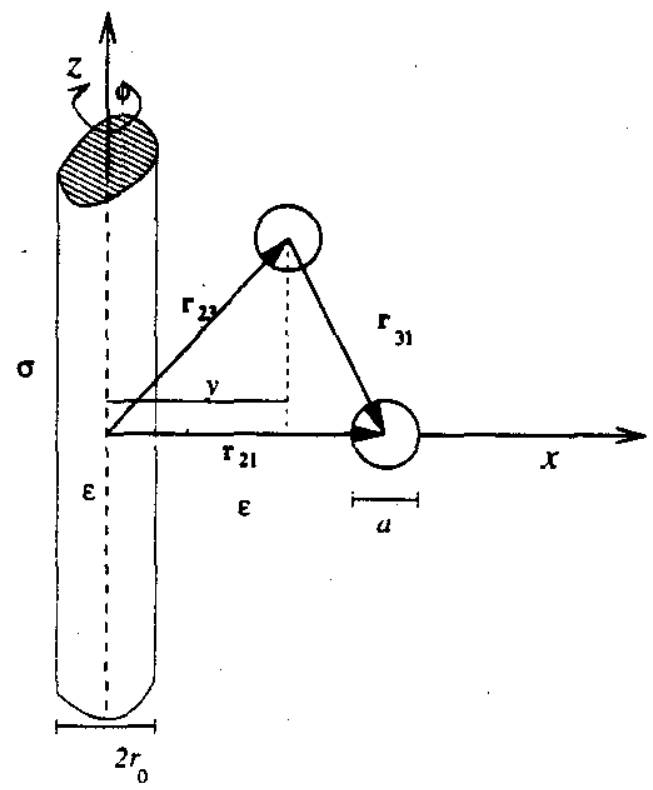

Figura 2.3: Representación esquemática el modelo usado en la deducción de la ecuación HNC/MSA, para una varilla de geometría cilíndrica inmersa en un electrolito tipo modelo primitivo restringido.

por

$$
\sum_{m=1}^{2} z_{m} \rho_{m}=0,
$$


donde $\rho_{m}$ y $z_{m}$ son la concentración y la valencia de la especie $m$. Para satisfacer la condición de campo eléctrico igual a cero en infinito, ia cual es necesaria para alcanzar equilibrio termodinámico, la carga sobre la varilla debe ser apantallada por un exceso de carga en el fluido, de tal forma que se debe satisfacer que:

$$
\lambda^{\prime} \equiv 2 \pi \int_{r_{0}+a / 2}^{\infty} \rho_{e l}(r) r \mathrm{~d} r=-\lambda, \quad \text { donde } \quad \rho_{e l}(r) \equiv e \sum_{m=1}^{2} z_{m} \rho_{m}(r)
$$

La concentración local de la especie $m$ se denota como $\rho_{m}(r)$. Como la carga de la varilla está uniformemente distribuida sobre su superficie, ésta tiene una densidad superficial de carga equivalente, $\sigma$, igual a $\sigma=\frac{\lambda}{2}$. . La ecuación de Ornstein-Zernike para fluidos inhomogéneos está dada por

$$
h_{\alpha j}\left(\mathbf{r}_{21}\right)=c_{\alpha j}\left(\mathbf{r}_{21}\right)+\sum_{m=1}^{2} \rho_{m} \int h_{\alpha m}\left(\mathbf{r}_{23}\right) c_{m j}\left(\mathbf{r}_{13}\right) \mathrm{d} v_{3}
$$

donde $h_{\alpha j}\left(\mathbf{r}_{21}\right)$ y $c_{\alpha j}\left(\mathbf{r}_{21}\right)$ son las funciones de correlación total y de correlación directa, entre las partícula 2 localizada en $\mathbf{r}_{2}$ y la partícula 1 en $\mathbf{r}_{1}$ de las especies $\alpha$ y $j$, respectivamente; $\mathbf{r}_{21} \equiv \mathbf{r}_{2}-\mathbf{r}_{1}$. En el esquema del método directo (ver capítulo 1), si la aproximación $\mathrm{HNC}$ es usada para la función de correlación directa entre la varilla y la especie $j$, la Ec. (2.3) se convierte en

$$
g_{\alpha j}\left(\mathbf{r}_{21}\right)=\exp \left\{-\beta u_{\alpha j}\left(\mathbf{r}_{21}\right)+\sum_{m=1}^{2} \rho_{m} \int h_{\alpha m}\left(\mathbf{r}_{23}\right) c_{m j}\left(\mathbf{r}_{13}\right) \mathrm{d} v_{3}\right\}
$$

Bajo este esquema, las funciones de correlación $h_{\alpha j}\left(\mathbf{r}_{21}\right)$ y $c_{\alpha j}\left(\mathbf{r}_{21}\right)$ corresponden a las funciones de correlación inhomogéneas de una partícula, $h_{j}(\mathbf{r})$ y $c_{j}(\mathbf{r})$, para las especies $j$ de un fluido bajo la influencia del campo externo producido por la varilla cilíndrica. La concentración local para la especie $j$ viene dada por $\rho_{j}(\mathbf{r})=\rho_{j} g_{j}(\mathbf{r})$, donde $g_{j}(\mathbf{r})$ es llamado el perfil reducido de concentración. Por lo que el perfil de concentración de carga está dado por

$$
\dot{\rho}_{\mathrm{el}}(r)=\sum_{m=1}^{2} q_{m} \rho_{m} g_{m}(r) \text { donde } q_{n}=z_{m} e .
$$

Para este modelo el potencial de interacción directe entre la varilla y la especie $j$ del fluido, $u_{\alpha j}(r)$, se puede separar en dos partes: el trrririro le interacción dura ion-varilla, $u_{\alpha j}^{*}(r), y$ el término de interacción electrostática, $u_{\alpha, i}^{\text {el }}(r)$. El primero toma en cuenta el hecho de que los iones no pueden deformar o pen trar la varilla cilíndrica, el cual viene dado por

$$
u_{\alpha j}^{*}(r)= \begin{cases}\infty & : \quad 0<r_{0}+a / 2 \\ 0 & : \quad \dot{r}>0+a /\end{cases}
$$


El segundo puede ser encontrado a partir de la ley de Gauss,

$$
-\beta u_{\alpha j}^{\mathrm{el}}(r)=\frac{4 r ; \beta}{\varepsilon} q_{i} \sigma r_{0} \ln (r) \quad\left(r>r_{0}\right) .
$$

con $\beta=1 / k_{B} T$. En la aproximac ón MSA la función de correlación directa para un electrolito tipo MPR tiene expresiones analíticas. Esta función puede ser escrita como

$$
c_{m j}(s)=-\beta u_{m j}^{\mathrm{el}}(s)+z_{m} z_{j} c_{\mathrm{d}}^{\mathrm{sr}}(s)+c_{\mathrm{s}}^{\mathrm{hs}}(s) .
$$

La expresión para esta función está dada en el apéndice B. Cuando usamos la cerradura MSA en la integral de la Ec. (2.4), se obtiene la ecuación HNC/MSA para un electrolito en presencia de una varilla cargada. Aprovechando la geometría cilíndrica del sistema y el hecho de que la función de correlación directa entre los iones depende únicamente de su distancia relativa $s \equiv\left|\mathbf{r}_{1}-\mathbf{r}_{3}\right|$, la Ec. (2.4) puede ser escrita como $[29,80]$

$$
\begin{aligned}
g_{j}(r)= & \exp \left\{\frac{4 \pi \beta}{\varepsilon} q_{i} \sigma r_{0} \ln (r)+\frac{2 \pi \beta q_{i}}{\varepsilon} \int_{r_{0}+a / 2}^{\infty} \rho_{\mathrm{el}}(y) \ln \left[\left(r^{2}+y^{2}+\left|r^{2}-y^{2}\right|\right) / 2\right] y \mathrm{~d} y\right. \\
& \left.+\int c^{\mathrm{hs}}(s) \rho_{\mathrm{cs}}(y) \mathrm{d} v_{3}+z_{i} \int c^{\mathrm{sr}}(s) \rho_{\mathrm{cd}}(y) \mathrm{d} v_{3}\right\}
\end{aligned}
$$

donde se ha definido

$$
\begin{aligned}
\rho_{T} & =\sum_{m=1}^{2} \rho_{m}, \\
\rho_{c s}(y) & =\sum_{m=1}^{2} \rho_{m} h_{m}(y), \\
\rho_{\text {cd }}(y) & =\sum_{m=1}^{2} z_{m} \rho_{m} h_{m}(y) .
\end{aligned}
$$

En coordenadas cilíndricas $\mathrm{d} v_{3}=r_{3} \mathrm{~d} r_{3} \mathrm{~d} z_{3} \mathrm{~d} \phi_{3} \mathrm{y}$

$$
s^{2}=i^{2}+r^{2}+y^{2}-2 r y \cos \phi,
$$

donde hemos llamado $y=r_{3}, z=z_{3} \quad \phi=\phi_{3}$. Integrando en coordenadas cilíndricas se obtiene

$$
\begin{aligned}
g_{j}(r)= & \exp \left\{\frac{4 \pi \beta}{\varepsilon} q_{i} \sigma r_{0} \ln (r)+\frac{2}{\varepsilon}-\frac{3}{\varepsilon}-q_{r_{0}+a / 2}^{\infty} \rho_{\mathrm{el}}(y) \ln \left[\left(r^{2}+y^{2}+\left|r^{2}-y^{2}\right|\right) / 2\right] y \mathrm{~d} y\right. \\
& \left.+\int_{r_{0}+a / 2}^{\infty} K(r, y) \rho_{\mathrm{cs}}(y) \mathrm{d} y+z_{i} \int_{r_{0}+a / 2}^{\infty} L(r, y) \rho_{\mathrm{cd}}(y) \mathrm{d} y+\rho_{T} A(r)\right\},
\end{aligned}
$$

donde

$$
K(r, y)=-4 y \int_{J_{1}}^{\phi_{0}}\left[c_{1} J_{0}+6 \eta c_{2} J_{1}+\frac{1}{2} \eta c_{3}\right] \mathrm{d} \phi
$$


para $|r-y| \leq a$ y $K(r, y)=0$ para $|r-y|>a$. Las constantes $\eta$ y $c_{i}$ están dadas en el apéndice $B$

$$
L(r, y)=\frac{4 e^{2} \beta y}{\varepsilon} \int_{0}^{\phi_{0}}\left[J_{2}+\frac{2 \Gamma J_{0}}{1+\Gamma a}+\frac{\Gamma^{2} J_{1}}{(1+\Gamma a)^{2}}\right] \mathrm{d} \phi
$$

para $|r-y| \leq a$ y $L(r, y)=0$ para $|r-y|>a . A(r)$ ha sido definida como

$$
A(r)=-\int_{0}^{r_{0}} K(r, y) \mathrm{d} y
$$

para $r \leq r_{0}+3 / 2 a$. En las ecuaciones anteriores

$$
\begin{aligned}
\phi_{0} & \equiv \cos ^{-1}\left[\frac{r^{2}+y^{2}-a^{2}}{2 r y}\right], \\
J_{0} & =\left(a^{2}-r^{2}-y^{2}+2 r y \cos \phi\right)^{2}, \\
J_{1} & =\frac{1}{2} a J_{0}+\frac{1}{2} S^{2} J_{2}, \\
J_{2} & =\ln \left(\frac{a+J_{0}}{S}\right), \\
J_{3} & =\frac{1}{4} a\left[a^{2}+\frac{3}{2} S^{2} J_{0}+\frac{3}{8} S^{4} J_{2}\right] \mathrm{y} \\
S & =\left(r^{2}+y^{2}-2 r y \cos \phi\right) .
\end{aligned}
$$

En la Ec. (2.10) podemos identificar el potencial electrostático promedio, de tal forma que puede reescribirse como

$$
g_{j}(r)=\exp \left\{-q_{j} \beta \psi(r)+\int_{r_{0}+a / 2}^{\infty} K(r, y) \rho_{\mathrm{cs}}(y) \mathrm{d} y+z_{i} \int_{r_{0}+a / 2}^{\infty} L(r, y) \rho_{\mathrm{cd}}(y) \mathrm{d} y+\rho_{T} A(r)\right\},
$$

donde $\psi(r)$ es el potencial electrostático promedio dado por

$$
\psi(r)=-\frac{4 \pi}{\varepsilon} \sigma r_{0} \ln (r)-\frac{\dot{2 \pi}}{\varepsilon} \int_{r_{0}+a / 2}^{\infty} \rho_{\mathrm{el}}(y) \ln \left[\left(r^{2}+y^{2}+\left|r^{2}-y^{2}\right|\right) / 2\right] y \mathrm{~d} y .
$$

En el límite de iones puntuales, i. e., $a \rightarrow 0, c_{\mathrm{d}}^{\mathrm{sr}}(s)$ y $c_{\mathrm{s}}^{\mathrm{hs}}(s) \rightarrow 0$ y la Ec. (2.9) se escribe como

$$
g_{m}(r)=\exp \left\{-\beta z_{m} e \psi(r)\right\}
$$

donde $\psi(r)$ está dado por la Ec. (2.14), la cual es solución de la bien conocida ecuación diferencial de Poisson-Boltzmann para iones puntuales alrededor de un electrodo cilíndrico cargado. i.e.,

$$
\frac{1}{r} \frac{\mathrm{d} v(r)}{\mathrm{d} r}+\frac{\mathrm{d}^{2} v(r)}{\mathrm{d} r^{2}}=-\frac{e}{\varepsilon} \sum_{m=1}^{2} z_{m} \rho_{m} \exp \left\{-\beta z_{m} e_{0} \psi(r)\right\}
$$

Por lo que la versión integral de la ecuación de Poisscn-Boltzmann es [80]

$$
g_{j}(r)=\exp \left\{\frac{4 \pi \beta}{\varepsilon} q_{j} \sigma r_{0} \ln (r)+\frac{2 \varepsilon_{j} \pi \beta}{\varepsilon}-\int_{r_{0}+a / 2}^{\infty} \rho_{\mathrm{el}}(y) \ln \left[\left(r^{2}+y^{2}+\left|r^{2}-y^{2}\right|\right) / 2\right] y \mathrm{~d} y\right\},
$$


que es la ecuación HNC/MSA en el límite de iones puntuales. La principal diferencia entre estas dos teorías se debe básicamente a las correlaciones por tamaño de los iones las cuales sí son tomadas, aunque sólo sea parcialmente, en la teoría HNC/MSA mientras que en la teoría de Poisson-Boltzmann son completamente ignoradas. Es bien sabido que las correlaciones por tamaño se vuelven importantes bajo condiciones de un fuerte acoplamiento entre los iones y el campo externo, iones polivalentes y una concentración alta del electrolito [29].

Las ecuaciones HNC/MSA y de Poisson-Boltzmann son resueltas numéricamente con técnicas eficientes de elemento finito [16, 102]. La solución de la Ec. (2.9) puede tomar un minuto en un procesador R1200 de una máquina SGI. En nuestros cálculos té́ricos usamos los mismos parámetros de entrada que en los cálculos de dinámica mo'ecular. Como un ejemplo, la concentración de sal es obtenida por

$$
\rho_{\mathrm{s}}=N_{\mathrm{s}} / L_{\mathrm{b}}^{3}
$$

donde $N_{\mathrm{s}}$ es el número de moléculas de sal usadas en la simulación. Sin embargo, debemos de remarcar que existen diferencias en los modelos usados en cada cálculo p. ej.: el potencial de interacción en ambos modelos. Mientras que en $H N C / M S A$ usamos particulas completamente duras, en los cálculos de dinámica molecular se usa un potencial con cierta suavidad. Ésto puede resultar en un volumen excluido diferente respecto al usado en los cálculos teóricos. Las diferencias que se introducen por este hecho serán discutidas en la presentación de los resultados.

\subsection{Detalles de los cálculos por dinámica molecular es- tocástica (DME)}

\subsubsection{Potenciales de interacción}

Para llevar a cabo la descripción del sistema es necesario especificar los potenciales de interacción del modelo, el cual toma en cuenta dos contribuciones: (i) el potencial de la interacción por volumen excluido, el cual previene a dos particulas de ocupar la misma posición y (ii) el término de interacción coulómbico de largo alcance.

Para el potencial de interacción por volumen excluido entre los iones se usa el siguiente potencial:

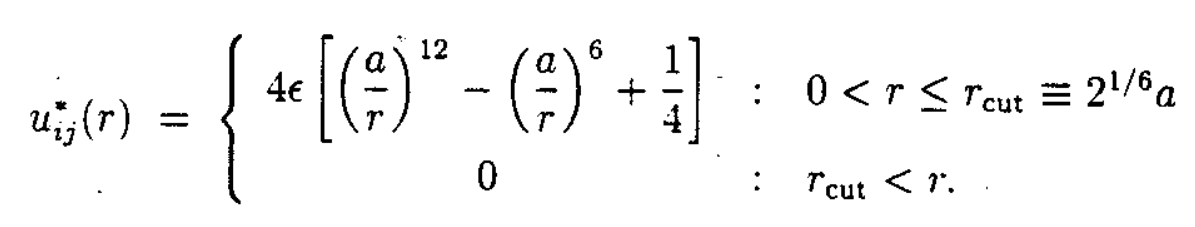

Sin eì corte. este potencial sería un potercial común tipo Lenard-Jones describiendo partículas de diámetro a $y$ un mínimo de potencial de profundidad $\epsilon$. Para aicanzar la 
repulsión, se corta el potencial en el mínimo y lo subimos de tal forma que vaya suavemente a cero. Dado que dos iones a una distancia de $a$ tiene una energía repulsiva de interacción $\epsilon$, hacemos la elección de $\epsilon=k_{\mathrm{B}} T$.

Para dar un radio mayor a la varilla que el de los iones, empleamos un potencial ionvarilla similar al de la Ec. (2.19), en el cual $r$ es reemplazado con $r-r_{\mathrm{s}}$. Este cambia la distancia de interacción rígida $r_{\mathrm{s}}$ hacia un radio mayor, el cual da una distancia de máximo acercamiento de $r_{\mathrm{s}}+a$. Dado que los iones tienen un diámetro de $a$ esto corresponde a un radio de la varilla, $r_{0}$, de $r_{0}=r_{\mathrm{s}}+a / 2$. En este caso $r$ es la coordenada cilíndrica.

La energía de interacción electrostática entre dos iones con carga $q_{i}$ y $q_{j}$, viene dada por

$$
u_{i j}^{\mathrm{el}}(r)=z_{i} z_{j} k_{\mathrm{B}} T \frac{\ell_{\mathrm{B}}}{r} \quad \text { donde } \quad \ell_{\mathrm{B}}=\frac{e^{2}}{s k_{\mathrm{B}} T},
$$

donde $z_{i}$ es la valencia de la especie $i$ y $e$ es la carga protónica elemental. Por definición la longitud de Bjerrum, $\ell_{\mathrm{B}}$, es la distancia a la cual dos cargas elementales tienen una energía electrostática de interacción de $k_{\mathrm{B}} T$. Con el valor de la constante dieléctrica del agua, $\varepsilon=78.5$, a temperatura ambiente, $T=298 \mathrm{~K}$, se obtiene un valor para la longitud de Bjerrum de aproximadamente $\ell_{\mathrm{B}}=7.14 \AA$. La densidad lineal de carga, $\lambda$, de la varilla es modelada poniendo unidades de carga a lo largo del eje de la varilla a una distancia $b=e / \lambda$. El número de cargas a lo largo de la varilla por unidad de longitud de Bjerrum es una medida adimensional importante de la densidad lineal de carga y a menudo es referida como el parámetro de Manning:

$$
\xi_{\mathrm{M}}=\frac{\lambda \ell_{\mathrm{B}}}{e}=\frac{\ell_{\mathrm{B}}}{b} .
$$

Su importancia radica en el hecho de que para $\xi_{\mathrm{M}}>1$ el fenómeno de condensación de los contraiones es observado [103] en sistemas que contienen únicamente contraiones, i. e., sin electrolito.

\subsubsection{La celda unitaria básica}

Con la finalidad de tener un sistema que puede considerarse como un sistema macroscópi$c o$, se genera una réplica de varias celdas unitarias, como ya ha sido mencionado en la sección 1.7.1. En las simulaciones que presentamos en este trabajo, se ha considerado una geometria que es computacionalmete simple. Se ha tomado una caja cúbica de longitud $L_{\mathrm{b}}$ en donde se ha colocado la molécula de ADN paralela a uno de los lados (ver la Fig. 2.4). La principal ventaja de esta aproximación es que tal sistema puede ser tratado con el método simple de sumas de Ewald o uno de sus variantes $[74,75,104,105]$. Esto permite una forma eficiente de calcular las interacciones electrostáticas de largo alcance. Con las condiciones periódicas empleadas en las simulaciones, la presencia de interacciones de largo alcance presenta complicaciones tanto matemáticas como técnicas. En las simulaciones se han empleado métodos eficientes de sumas aceleradas de Ewald, con métodos de la transformada rápida de Fourier (FFT), el algoritmo $\mathrm{P}^{3} \mathrm{M}$, el cual escala casi linealmente con el número de cargas $[74,75,105]$. 


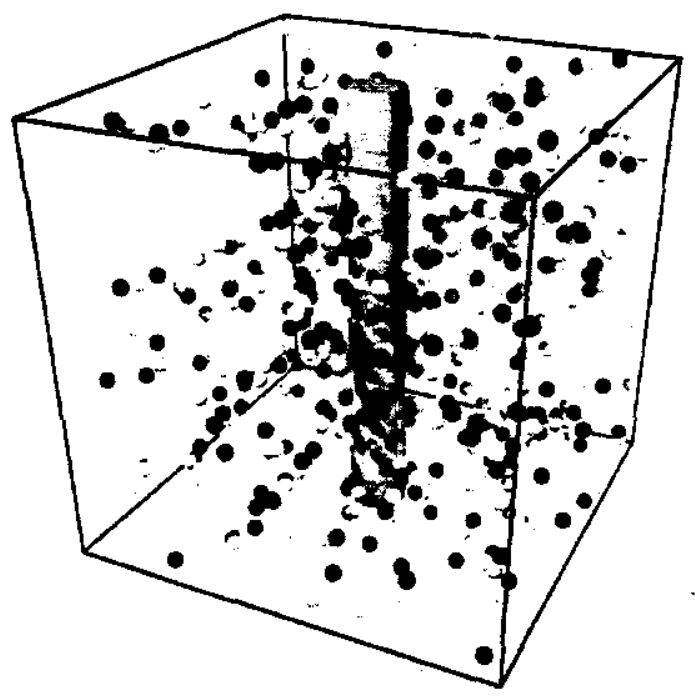

Figura 2.4: Una varilla de longitud $L_{\mathrm{b}}$ colocada de forma paralela a uno de los lados de la celda cúbica de longitud $L_{\mathrm{b}}$ nos da un arreglo cúbico infinito de varillas infinitamente largas bajo una replicación periódica de la caja cúbica. Las partículas oscuras representan los contraiones ( 0 iones positivos), mientras que las partículas claras son los iones negativos. Este dibujo en particular, muestra un sistema que contiene 36 contraiones divalentes, 220 iones positivos y 220 iones negativos de sal.

\subsubsection{Mapeo de los parámetros a una molécula de ADN}

Como siguiente paso, hemos mapeado explícitamente los parámetros a un sistema de moléculas de ADN en solución acuosa. Esto incluve el diámetro iónico $a$, el radio de la varilla cilíndrica $r_{0}$, la longitud característica de Bjerrum $\ell_{B}$ y la densidad lineal de carga $\lambda$. Nuestra elección es presentada en la Tabla 2.1. La simulación molecular del sistema, se ha realizado usando un termostato tipo Langevin para llevar al sistema a un estado canónico, i. e, de energía constante [106\}. La parte real e imaginaria del espacio de Fourier de la energía electrostática fue usada para verificar la condición de equilibrio. Los observables que aquí presentamos fueron originados al promediar aproximadamente sobre unas 1000 configuraciones independientes.

\subsection{Resultados}

La discusión de resultados la llevaremos a cabo en términos del potencial electrostático promedio $w(r)$. los perfiles reducidos de concentración, $g_{m}(r)$, y la función de distribución de carga. la cual queda definida por

$$
P(r)=\frac{1}{|\lambda|} \int_{r_{0}}^{r} \mathrm{~d} \bar{r} 2 \pi \bar{r} \rho_{e l}(\bar{r}) .
$$




\begin{tabular}{lccc}
\hline \hline parámetro & símbolo & valor & valor en unidades LJ \\
\hline \hline diámetro iónico & $a$ & $4.25 \AA$ & $a$ \\
valencia iónica & $z$ & 2 & 2 \\
radio de la varilla & $r_{0}$ & $7.86 \AA$ & $1.85 a$ \\
densidad de carga lineal (ADN) & $\lambda$ & $-e_{0} / 1.7$ & $-2.5 e_{0} / a$ \\
longitud de Bjerrum (en agua & $\ell_{\mathrm{B}}$ & $7.14 \AA$ & $1.68 a$ \\
parámetro de Manning & $\xi_{\mathrm{M}}$ & 4.2 & 4.2 \\
tamaño de la caja & $L_{\mathrm{b}}$ & $122.4 \AA$ & $28.8 a$ \\
radio de una celda correspondiente & $R$ & $69.1 \AA$ & $16.2 a$ \\
temperatura & $T$ & $298 \mathrm{~K}$ & $\epsilon / k_{\mathrm{B}}$ \\
\hline
\end{tabular}

Tabla 2.1: Parámetros del sistema usados en la simulación de la molécula de ADN en solución

Esta función, que es fundamental en nuestra discusión del sobrecargado, mide la cantidad de carga acumulada entre la superficie de la varilla y una superficie cilíndrica imaginaria de radio $r$, medido desde el centro de la varilla. En gran parte de nuestra discusión de resultados se presentan comparaciones entre los resultados teóricos y los de simulación, con la finalidad de verificar la validez de los resultados de HNC/MSA. Por otra parte, algunos de los mecanismos físicos que conducen al sobrecargado son evidenciados y discutidos.

La Fig. 2.5 muestra los resultados de HNC/MSA y DME para la función $P(r)$ y $\psi(r)$, para cinco densidades de carga superficial diferentes, esto quiere decir, cinco valores diferentes del parámetro de Manning el cual se ha incrementado sucesivamente. De los resultados.que se presentan, uno de ellos tiene un valor del parámetro de Manning menor que el de la molécula de $\operatorname{ADN}$ (el cual es de $\xi_{M}=4.2$ ) y para tres de ellos es mayor. La función $P(r)$ presenta sobrecargado, i.e., a cierta distancia, la carga de la varilla no solamente es compensada sino que es sobrecompensada. Este hecho se manifiesta en la presencia de máximos en la función $P(r)$, los cuales incrementan su intensidad y se localizan más cercanos a la superficie del cilindro a medida que se incrementa la densidad de carga de la varilla. Como ha sido puntualizado con anterioridad $[29,82]$, el efecto de sobrecargado implica un cambio de dirección del campo eléctrico local. Nuestros resultarios de DME y HNC/MSA son consistentes con tal hecho.

En el lado derecho de la Fig. 2.5 se muestran las gráficas del potencial electrostático promedio, $\psi(r)$. Podemos observar que $\langle\cdot(x)$ presenta valores negativos y además se muestra la presencia de un mínimo, lo que indica un cambio de pendiente en $\psi(x)$. El minimo se localiza más cerca de la superficie de la varilla e incrementa su intensidad, a medida que se incrementa la densidad de carga. El cambio de pendiente representa un cambio en la dirección del campo eléctrico local. Es decir, después del mínimo de potencial el campo eléctrico cambia de direcciór de positivo a negativo. En la región donde el campo eléctrico invierte su dirección es donde ocurre sobrecargado. Es sabido que HNC/MSA da resultados más fiables que la teor a de Poisson-Boltzmann en sistemas que contienen una alta concentración de electrolito, iones polivalintes $\mathrm{y} / \mathrm{o}$ para fluidos bajo la influencia 

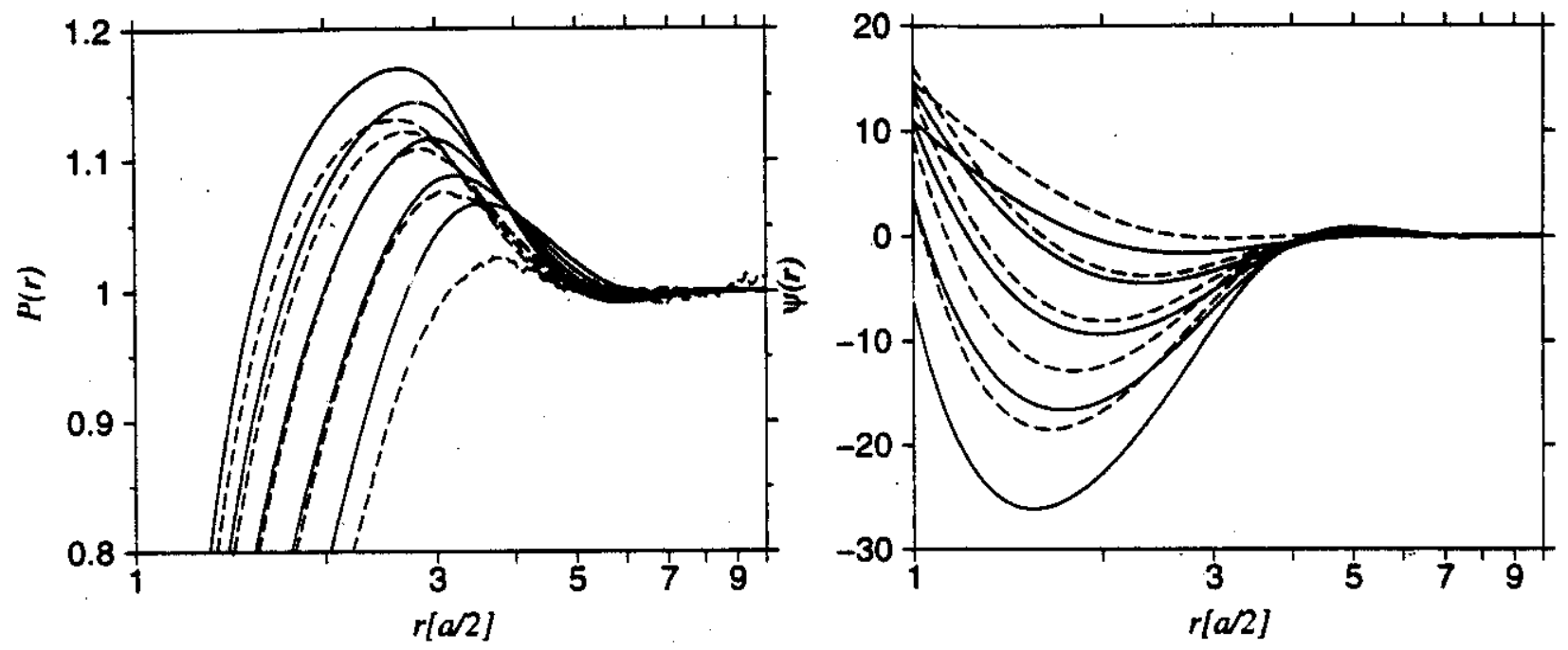

Figura 2.5: (a)Función de distribución de carga $P(r)$ y (b) potencial electrostático promedio $\psi(r)$ para los parámetros de la tabla 2.1. El electrolito es una sal divalente a una concentración de $0.49 \mathrm{M}$. La distancia $r$ está medida respecto a la superficie del cilindro y está dada en unidades de radios iónicos. Las líneas sólidas e intermitentes corresponden a los cálculos de HNC/MSA y de DME, respectivamente. Las diferentes curvas corresponden a valores distintos del parámetro de Manning, $\xi_{\mathrm{M}}=2.1,4.2,6.3,8.4,10.5$, el cual corresponde a las densidades superficiales de carga de $\sigma=0.095,0.190,0.286,0.381,0.476 \mathrm{C} / \mathrm{m}^{2}$, respectivamente. En las gráficas de $P(r)$, la densidad superficial de carga se incrementa de abajo hacia arriba, mientras que en el potencial electrostático promedio se incrementa de arriba hacia abajo. El valor de $\xi_{\mathrm{M}}=4.2\left(\sigma=0.190 \mathrm{C} / \mathrm{m}^{2}\right)$ corresponde a la molécula de ADN.

de campos externos muy fuertes $[69,84,91]$. Esto se debe a que HNC/MSA, aunque de manera parcial, toma en cuenta las correlaciones debidas al tamaño de los iones las, cuales son completamente ignorados en la teoría de PB. Por lo que con la teoría de PB no se obtiene el efecto de sobrecargado. Se puede ver que la teoría $\mathrm{HNC} / \mathrm{MSA}$ en verdad predice sobrecargado, aunque el efecto se ve sobreestimado respecto a los resultados obtenidos por medio de D.ME. El acuerdo cualitativo (ver Fig. 2.5) entre los resultados de la teoría H.NC MSA y los resultados de DME es excelente. El acuerdo cuantitativo es bueno y es particularmente bueno para los cálculos correspondientes a la molécula de ADN. Más adelante vamos a discutir las causas del desacuerdo cuantitativo entre los resultados de H.YC/MSA y DME.

De acuerdo con la definición de la densidad superficial de carga, $\sigma$, se pueden tener distintos valores de $\sigma$ para el mismo valor de $\lambda$. De esta forma, para un mismo valor dado del parámetro de Manning, $\xi_{M}$, dos polielectrolitos cilírdricos con diámetros diferentes producirán diferentes estructuras de la doble capa eléctrica. En la Fig. 2.6 se presentan los resultados de HNC/MSA para distintas varillas cor: el misıno valor del parámetro de Manning, pero con distintos radios $r_{0}$. Estos cálculos muestran diferentes perfiles 


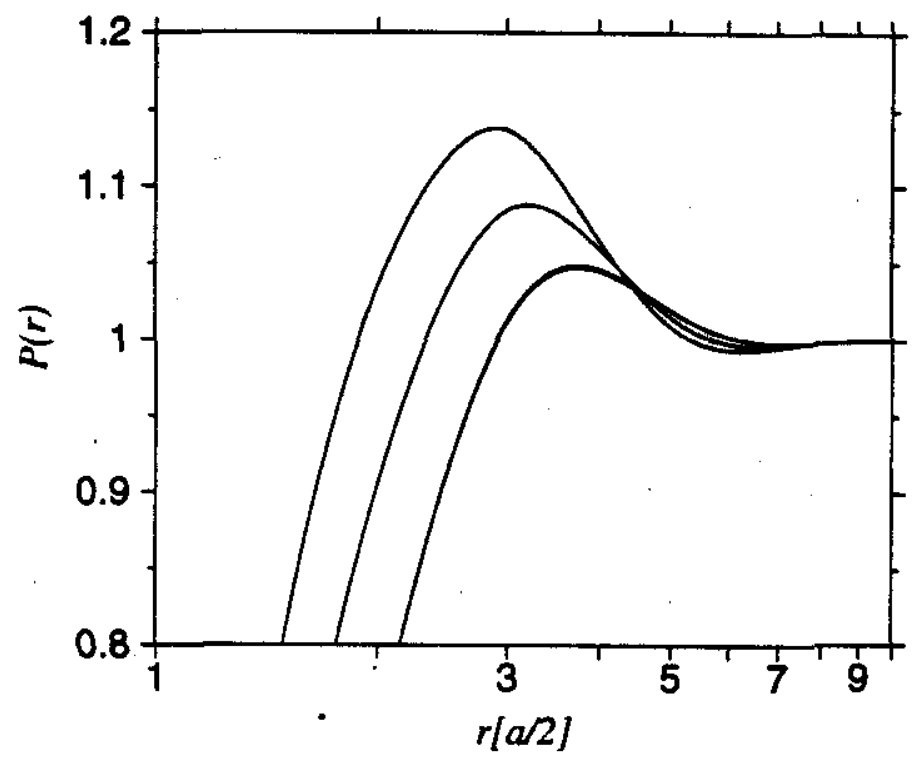

Figura 2.6: Función de distribución de carga obtenida de HNC/MSA, para un electrolito tipo MPR en contacto con una varilla cilíndrica cargada. La distancia $r$ es medida con respecto a la superficie del cilindro en unidades de radios iónicos. El electrolito es una sal divalente, a una concentración de $0.49 \mathrm{M}$. Los resultados corresponden a diferentés. pero manteniendo constante el valor del parámetro de Manning en un valor de $\xi_{M}=4.2$. De arriba hacia abajo los radios del cilindro son de $r_{0}=3.86 \AA, 7.86 \AA, 110 \AA y=1200 \AA$, los cuales corresponden a unas densidades superficiales de carga de $\sigma=0.388 \mathrm{C} / \mathrm{m}^{2}, 0.190,10^{-2} \mathrm{C} / \mathrm{m}^{2}$, $10^{-3} \mathrm{C} / \mathrm{m}^{2}$, respectivamente. Las curvas para $r_{0}=110 \AA$ y $r_{0}=1200 \AA$ son prácticamente indistinguibles.

de la función de distribución de carga $P(r)$. Esto ilustra el hecho de que el parámetro de Manning, por sí solo, no es suficiente para especificar la estructura iónica alrededor de macroiones cilindricos. Dado que la estructura iónica depende directamente de la magnitud del campo, es más adecuado usar la densidad de carga superficial del cilindro, $\sigma$, la cual es una medida directa del campo eléctrico que penetra dentro de la solución. Sin embargo, $\xi_{\mathrm{M}}$ y $\sigma$ están relacionados simplemente por $\xi_{\mathrm{M}}=2 \pi r_{0} \sigma \ell_{\mathrm{B}} / e$ [ver Ec. (2.21)]. Este hecho no está reconocido en la literatura.

En la Fig. 2.7 mostramos los resultados de HNC/MSA y DME para los perfiles reducidos de concentración, para $\sigma=0.190 \mathrm{C} / \mathrm{m}^{2}$ y $\sigma=0.49 \mathrm{C} / \mathrm{m}^{2}\left(\xi_{\mathrm{M}}=4.2\right.$ y $\xi_{\mathrm{M}}=10.5$, respectivamente), manteniendo el mismo valor de los demás parámetros que en la Fig. 2.5. Las oscilaciones en los perfiles de concentración aparecen como una consecuencia de las correlaciones inducidas por $\mathrm{t}$ l tamaño de los iones y son una manifestación del sobrecargado. El primer máximo, ot servado en el perfil de concentración reducido de los coiones, implica una concentración de éstos por arriba de su concentración de bulto e indica que la interacción efectiva ion-pared es atractiva a los coiones. Podemos notar que 

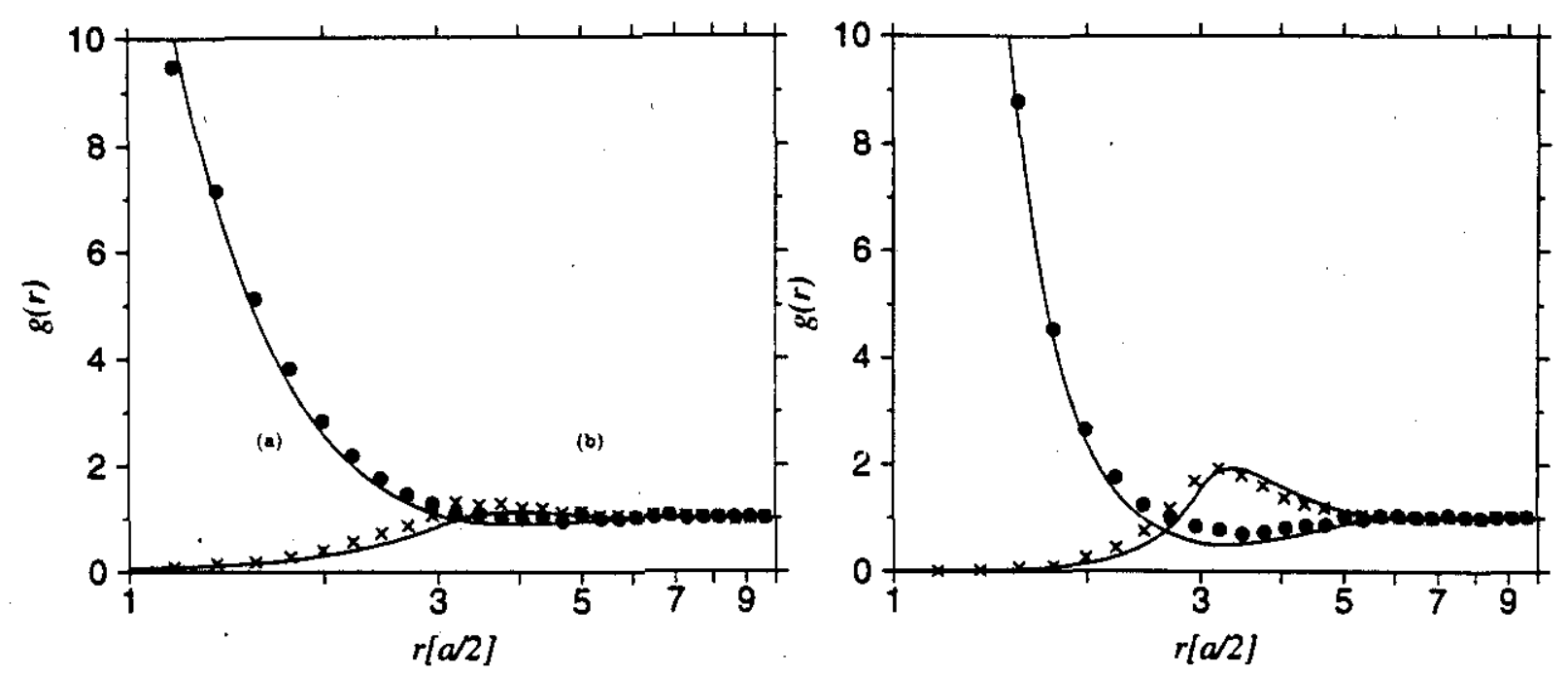

Figura 2.7: Perfil reducido de concentración, $g_{i}(r)$, para un electrolito en contacto con unavarilla cilíndrica cargada. El electrolito es una sal 2:2, a una concentración de $0.49 \mathrm{M}$. La distancia, $r$, está medida con respecto a la superficie del cilindro en unidades de radios iónicos. Los parámetros del sistema se encuentran dados en la Tabla 2.1. La Fig. 2.7a muestra los resultados correspondientes a $\xi_{\mathrm{M}}=4.2\left(\sigma=0.190 \mathrm{C} / \mathrm{m}^{2}\right)$, mientras que la Fig. $2.7 \mathrm{~b}$ muestra los resultados para $\xi_{\mathrm{M}}=10.5\left(\sigma=0.49 \mathrm{C} / \mathrm{m}^{2}\right)$ : Los puntos y cruces son las funciones de distribución obtenidas por medio de DME para los contraiones y coiones, respectivamente. La línea sólida son los resultados de HNC/MSA.

la magnitud de las oscilaciones es más fuerte a medida que se incrementa la intensidad del campo eléctrico así como el hecho de que se localizan cada vez más cercanas a la superficie del cilindro. Se puede apreciar que los resultados de la teoría HNC/MSA sobreestiman el valor de contacto de los perfiles de concentración respecto a las predicciones de dinámica molecular. Las diferencias entre las predicciones de HNC/MSA y DME pueden ser asociadas con dos hechos: (i) la diferencia del potencial repulsivo usada en HNC/MSA y DME y (ii) el hecho de que HNC/MSA es una teoría aproximada, por lo que no considera propiamente todas las correlaciones.

Otro fenómeno importante es obser vado en el comportamiento del potencial electrostático promedio, en el lado derecho de la Fig. 2.5. En lo que concierne al potencial en el punto en que los iones se encliestrín a la distancia de máximo acercamiento con la varilla

$$
\zeta=\psi^{\prime}\left(r_{0}+a / 2\right)
$$

el cual es identificado como el potencial- $\zeta$ usado en los cálculos de electroforesis, y que es de gran importancia en las teorías pare e' celculc de las movilidades electroforéticas $[35,107]$. En la teoria de Poisson-Boltzmann paré una varilla inmersa en una solución iónica, y su 
Figura 2.8: Gráfica del potencial $\zeta \equiv$ $\psi\left(r_{0}+a / 2\right)$ como una función de la densidad de carga superficial $\sigma$, para un cilindro inmerso en un electrolito $2: 2$ a una concentración de $0.49 \mathrm{M}$. Los parámetros del modelo están dados en la Tab. 2.1. La línea discomtinua es la predicción de la teoría de PB, los puntos riegros son los resultados de DME y la linea sólida son los resultados de la teoría HNC/MSA.

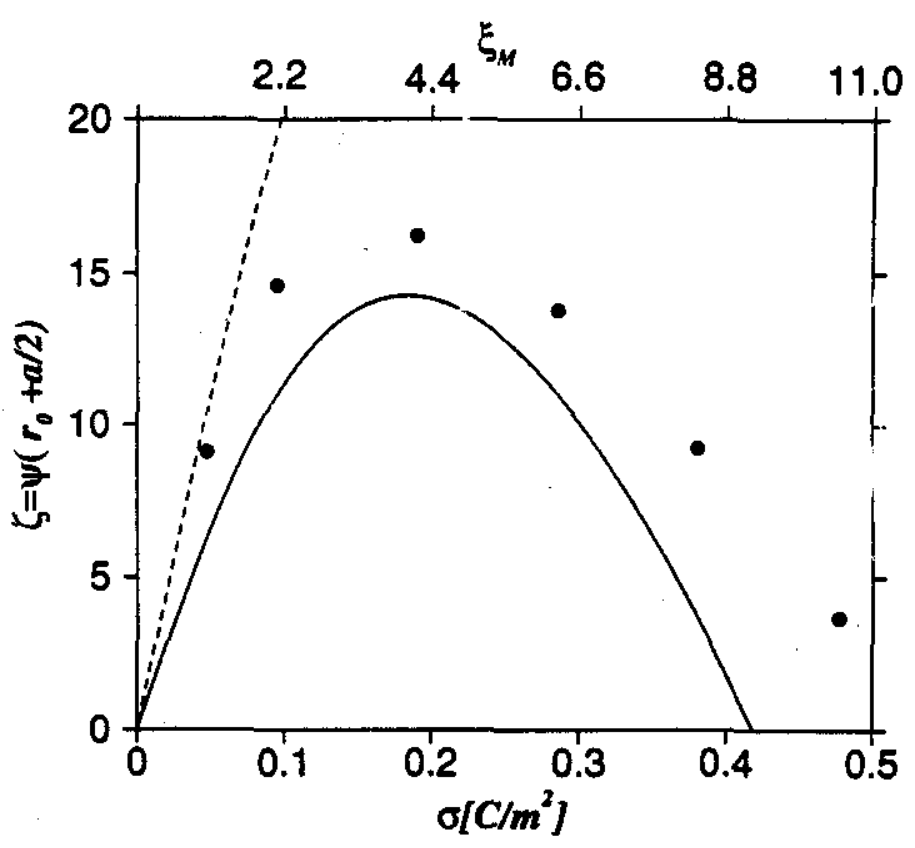

versión lineal, el potencial- $\zeta$ depende monotónicamente de $\xi_{\mathrm{M}}[29]$. Sin embargo, en la Fig. 2.5 se muestrà que al incluir las correlaciones por tamaño, $\psi(r)$, puede no ser monotónico e inclusive puede volverse negativo. Esto cambia la relación típica que se tenía entre $\zeta$ y $\xi_{\text {M. }}$. La tabla 2.2 contiene varias predicciones para el potencial $\zeta$ como una función del parámetro $\xi_{\mathrm{M}}$, así como algunos resultados obtenidos de los cálculos de simulación por DME. Una ilustración gráfica de estos datos se presenta en la Fig. 2.8.

En efecto, se encuentra que $\zeta$ primero se incrementa con $\xi_{\mathrm{M}}$, pero para cierto valor de $\xi_{M}, \zeta$ empieza a decrecer lo que finalmente lo vuelve negativo. Mientras que las teorias no lineal y lineal de Poisson-Boltzmann coinciden con los datos de simulación para valores pequeños de $\sigma$ (lo cual es un hecho fortuito), éstas fallan completamente al predecir el comportamiento no monotónico y el máximo de la función, el cual aparece para valores de $\xi_{\mathrm{M}}$ relativamente pequeños. La teoría $\mathrm{HNC} / \mathrm{MSA}$ captura este efecto, pero sobreestima el valor del potencial $\zeta$. Existen comparaciones que se han realizado anteriormente, para el potencial $\zeta$ como una función cie $\sigma$, entre HNC/MSA y datos de Monte Carlo para una superficie plana cargada y macroiones esféricos cargados, inmersos en un electrolito $[69,84,101]$. En estas geometrías, para electrolitos $1: 1$ y 2:2, a una concentración de $0.5 \mathrm{M}$, como en la Fig. 2.8, el acuerdo entre HNC/MSA y MC es excelente. De esta forma, podemos argumentar que el desacuerdo en la Fig. 2.8 puede asociarse en parte con el potencial repulsivo usado en hunstros cálculos de DME, los cuales no tienen un límite de contacto claramente definido encre los iones y el cilindro. Este hecho es importante dado que el potencial $\zeta$ es evaluado exactamente ahí.

Para macroiones planares y esféricos, los resultados de PB para las curvas de $\zeta$ vs. $\sigma$, se encuertran siempre por encima de las curvas de HNC/MSA y MC, lo cual es consistente con nuestra presente observaciion. Para una carga dada de la varilla y diámetro, para 


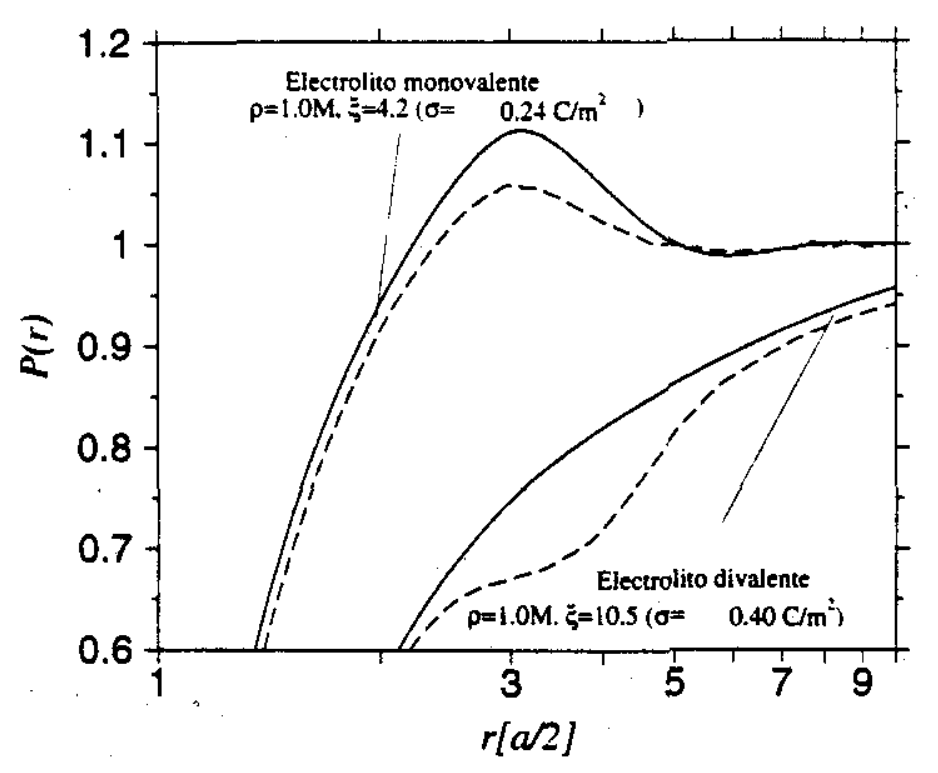

Figura 2.9: Función de distribución de carga obtenida de HNC/MSA (línea sólida) y DME (linea punteada), para un electrolito tipo MPR en presencia de una varilla cilindrica cargada, con la cual los iones tienen una distancia de máximo

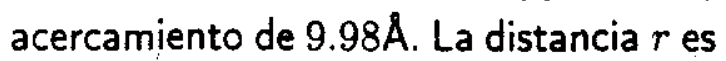
medida con respecto a la superficie del cilindro en unidades de radios iónicos. En esta gráfica mostramos una comparación entre dos sistemas uno de los cuales presenta un alto volumen excluido mientras que el ocro presenta un bajo volumen excluido. El sistema donde ocurre sobrecargado corresponde a un electrolito monovalente con $\rho=1.0 \mathrm{M}$, $\xi_{\mathrm{M}}=4.2\left(\sigma=0.240 \mathrm{C} / \mathrm{m}^{2}\right)$, y iones de diámetro $(a=7.4 \AA)$; mientras que el sistema donde no se observa sobrecargado corresponde a un electrolito divalente con una concentración de $\rho=0.5 \mathrm{M}$, $\xi_{\mathrm{M}}=10 . \tilde{s}\left(\sigma=0.40 \mathrm{C} / \mathrm{m}^{2}\right)$, y iones pequeños de diámetro $a=1.0 \AA)$.

cualquier conjunto de parámetros del electrolito, un valor más alto del potencial $\zeta$ siempre implica una menor adsorción de contraiones [29]. De esta forma, un potencial repulsivo suave. como en nuestros cálculos de D.ME. permite a los contraiones acercarse más a la varilla. Esto produce un valor más alto del potencial $\zeta$ en nuestros cálculos de DME al compararlos con los resultados de HNC MSA. En el caso de la teoría de PoissonBoltzmann. el no tomar en cuenta las correlaciones por tamaño, no permite una mavor concentración de contraiones sobre la varilla para producir sobrecargado.

En todos los casos el acuerdo cualitativo es muy bueno. El éxito de la teoría HNC/MSA indica que las correlaciones por tamaño son responsables sobrecargado y la curvatura del potencial $\zeta$. De esta forma, podemos ver que el sobrecargado está relacionado con el efecto de volumen excluido. Si estos efectos no son considerados (como en el caso de la teoría de Poisson-Boltzmann) o despreciables. el sobrecargado no se presenta, aun para iones polivalentes. Por otro lado. el sobrecargado puede ocurrir en un sistema con un alto volumen excluido incluso para iones monovalentes. Para ilustrar este hecho. hemos simulado dos sistemas para los cuales la máxima distancia de acercamiento a la superficie de la varilla cargada es de $9.98 . \dot{A}$. Para el primer caso (electrolito monovalente) se trata de una varilla con un parámetro de carga de $\xi_{M}=4.2\left(\sigma=0.240 \mathrm{C} / \mathrm{m}^{2}\right)$ y está inmersa en un electrolito monovalente a una concentración de $1.0 \mathrm{M}$ con iones de 
diámetro $a=7.4 \AA$. El segundo sistema (electrolito divalente) tiene un parámetro de carga de $\xi_{\mathrm{M}}=10.5\left(\sigma=0.40 \mathrm{C} / \mathrm{m}^{2}\right)$ y está inmerso en un electrolito divalente a una concentración de $0.5 \mathrm{M}$, con iones de diámetro $a=1.0 \AA$. Los resultados se muestran en la Fig. 2.9, donde graficamos la función de distribución de carga. El sistema con iones grandes y monovalentes presenta sobrecargado con ambos cálculos, HNC/MSA y DME. La localización de los picos coincide, mientras que observamos pequeñas diferencias en su altura. Curiosamente el sistema con iones divalentes pequeños no presenta signo alguno de sobrecargado, aunque el parámetro de carga para el sistema es aún mayor. El hombro en la función de distribución de carga obtenido de DME, indica que la segunda capa iónica es casi neutral. Una explicación tentativa es la siguiente: Los iones pequeños tienen una energía de atracción de casi $30 k_{\mathrm{B}} T$, por lo tanto, el electrolito está en forma de pequeños cúmulos neutros. Esta explicación es oportada por inspección visual de una configuración de DME. La mayoría de estos ci mulos neutros son polarizables y por lo tanto tienden a acumularse en la dirección del campo eléctrico que rodea la varilla. De esta forma, los contraiones libres, i. e., aquellos què: neutralizan la carga sobre la varilla de ninguna forma pueden producir sobrecargado. De manera consistente los cálculos de HNC/MSA tampoco presentan sobrecargado.
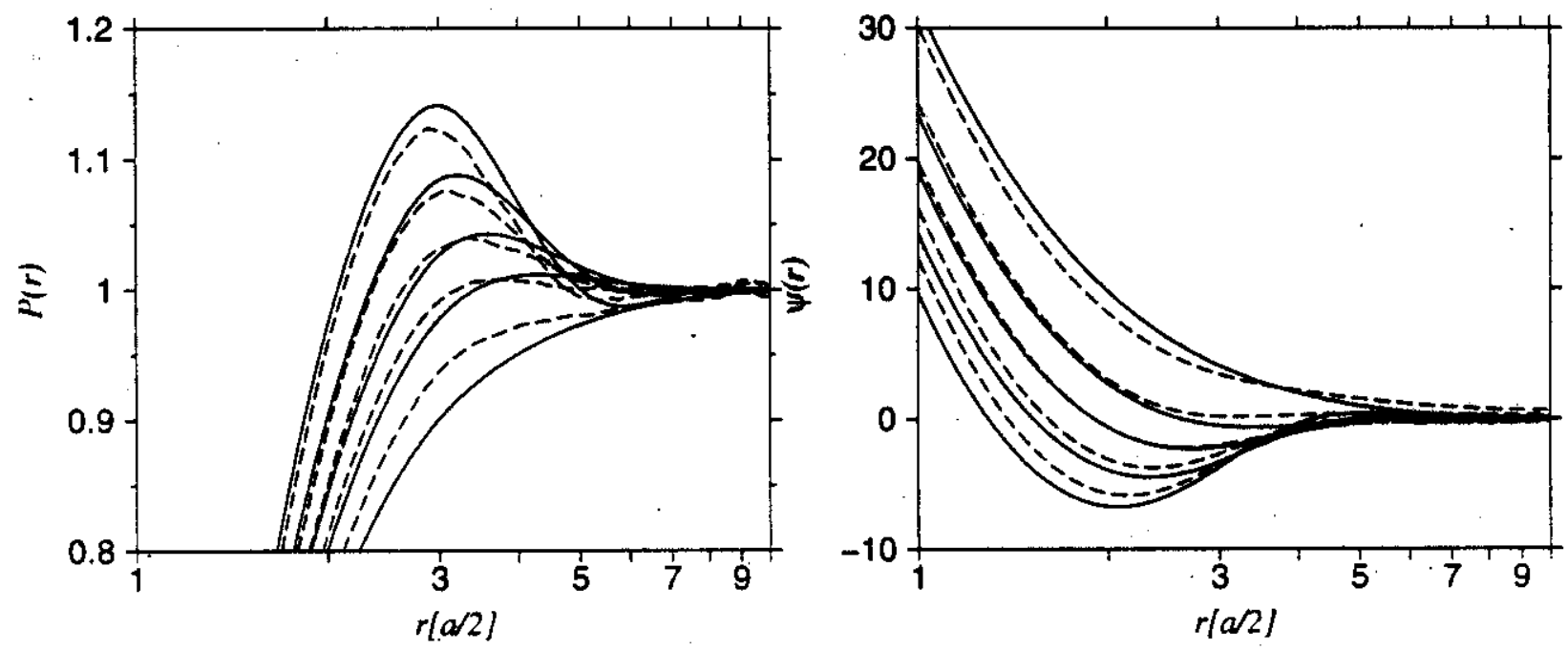

Figura 2.10: Resultados para la función de distribución de carga $P(r)$ (izquierda) y potencial electrostático promedio $\psi(r)$ (derecha), para los sistemas de la tabla 2.1 con valor del parámetro de Manning de $\xi_{\mathrm{M}}=4.2$. La distancia $r$ es medida con respecto a la superficie del cilindro en unidades de radios iónicos. El significado de las líneas es el mismo que en la Fig. 2.5. Las curvas corresponden a diferentes concentraciones del electrolito, $\rho_{\mathrm{s}}=0.12,0.24,0.34,0.49,0.68 \mathrm{M}$. En las funciones de distribución de carga la concentración del electrolito se incrementa de abajo hacia arriba, en el potencial electrostátiço promedio ésta se incrementa de arriba hacia abajo. 


\begin{tabular}{lllll}
\hline \hline$\xi_{\mathrm{M}}$ & $\zeta_{\mathrm{LPB}}[\mathrm{mV}]$ & $\zeta_{\mathrm{PB}}[\mathrm{mV}]$ & $\zeta_{\mathrm{HNC}}[\mathrm{mV}]$ & $\zeta_{\mathrm{MD}}[\mathrm{mV}]$ \\
\hline 1.05 & 10.52 & 10.28 & 6.262 & 9.116 \\
2.1 & 21.04 & 19.48 & 10.99 & 14.62 \\
4.2 & 42.09 & 33.32 & 14.27 & 16.25 \\
6.3 & 63.13 & 42.96 & 11.08 & 13.80 \\
8.4 & 84.17 & 50.16 & 3.771 & 9.263 \\
10.5 & 105.2 & 56.03 & -6.219 & 3.675 \\
\hline
\end{tabular}

Tabla 2.2: Potencial $\zeta$ para una varilla tipo ADN (ver Tab. 2.1), inmersa en un electrolito 2:2 a una concentración de $0.49 \cdot \mathrm{M}$, como una función del parámetro de Manning $\xi_{\mathrm{M}}$. Las cuatro predicciones son: la teoría lineal de $\mathrm{PB}$, la teoría de PB, HNC/MSA y simulación por dinámica molecular. El error de los datos de simulación es estimado del orden del $2 \%$. La Fig. 2.8 muestra la gráfica de los datos.

Finalmente, uno puede preguntarse cómo es que el efecto de sobrecargado depende de la concentración de sal. Obviamente en ausencia de sal no puede haber sobrecargado, ya que aun una condensación completa de los contraiones únicamente neutralizaría la carga de la varilla. Sin embargo, no es claro aún, cómo es que solamente por adición de sal aparece el sobrecargado. La Fig. 2.10 muestra los resultados de HNC/MSA' y DME para la función de distribución de carga, $P(r)$, y el potencial electrostático promedio, $\psi(r)$, para una varilla cargada, con los parámetros de la molécula de ADN, inmersa en un electrolito divalente en diferentes concentraciones de electrolito. El sobrecargado se observa solamente a una concentración de sal lo suficientemente alta. Esto puede ilustrarse definiendo $r_{1}$, como la distancia a la cual el sobrecargado comienza, i.e.,

$$
r_{1}=\min \{r: P(r)=1\} .
$$

La Fig. 2.11 ilustra el valor de $r_{1}$ predicho por DME así como la predicción de HNC/MSA, como una función de la concentración del electrolito. $\rho_{\mathrm{s}}$. Claramente, $r_{1}$ debe aumentar con la disminución de $\rho_{\mathrm{s}}$, dado que el ancho de la DCE se incrementa y consecuentemente se reduce el sobrecargado. En efecto, HNC/MSA predice que $r_{1}\left(\rho_{\mathrm{s}}\right)$ diverge a una concentración finita de $\rho_{\mathrm{s}}^{\infty} \approx 0.18 \mathrm{M}$ lo que corresponde a una longitud de Debve de $3.59 \AA$, o aproximadamente 200 moléculas de sal en la caja de simulación.

Para concentraciones menores, los valores de $r_{1}$ obtenidos por simulación caen ligeramente por debajo de la predicción de HNC MSA. pero esto no es una característica general. En la Fig. 2.10 puede verse que a mavor sobrecargado, la teoria HNC/MSA predice un valor de $r$, ligeramente menor que en la simulación. Sin embargo al disminuir la concentración, el tamaño finito de la celda de simuiación se vuelve relevante ya los iones alcanzan el valor de $r_{1}$ en el borde de la celcla y no en infinito. Una reducción en la cantidad de sal a un tamaño fijo de la selda debe necesariamente conducir a valores. más pequeños de $r_{1}$ que en el caso de la teoría HNC . US.A, ya que $r_{1}$ no puede divergiren una celda de tamaño finito. Por otro lado, por razones técnicas, en los cálculos de 
Figura 2.11: Radio $r_{1}$ de la Ec. (2.24) a ia cual el sobrecargado comienza, como una función de la concentración de electroli :o $\rho_{s}$; para una varilla con los parámetros de la molécula de ADN $\left(r_{0}=7.86\right.$ y $\sigma=$ $\left.0.190 \mathrm{C} / \mathrm{m}^{2}\right)$. La distancia $r_{1}$ es medida con respecto a la superficie del cilindro en unidades de radios iónicos. Los puntos negros corresponden a los resultados de DME, mientras que la línea sólida es la predicción de HNC/MSA.

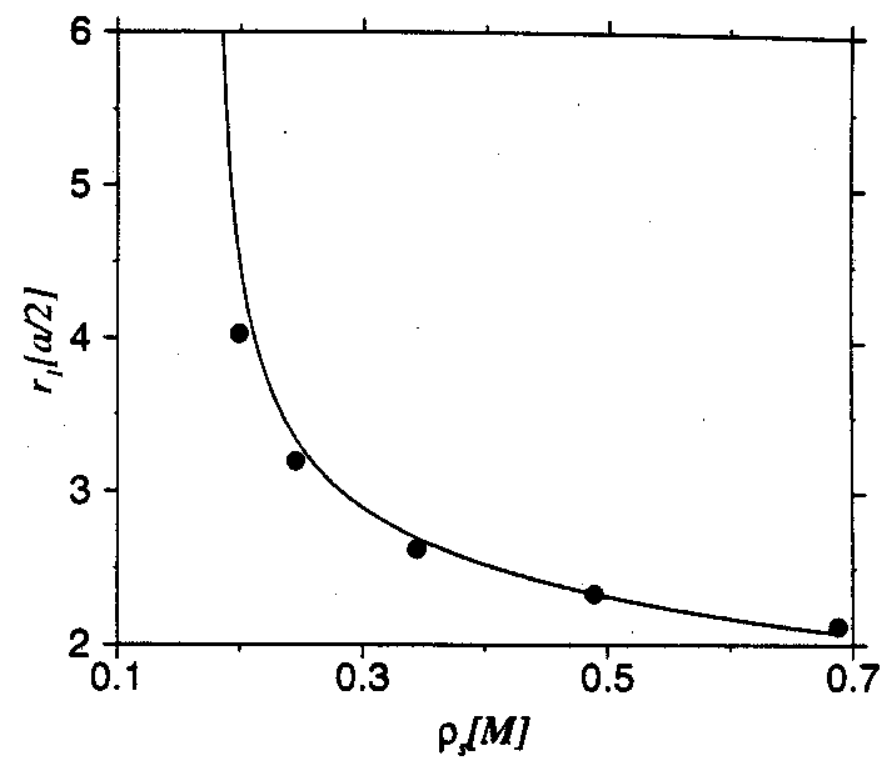

DME el tamaño de la celda no puede incrementarse indefinidamente. De cualquier forma, nuestros resultados de DME no han detectado sobrecarga para concentraciones menores de $\rho_{\mathrm{s}} \leq 0.2 \mathrm{M}$, lo que es un excelente acuerdo con la predicción de HNC/MSA, para una cantidad mínima de sal requerida para el sobrecargado. Este resuldado, así como el discutido en la Fig. 2.9 sientan el antecedente de la importancia de la concentración así como del tamaño iónico en el sobrecargado.

\subsection{Conclusiones de capitulo}

En este capítulo hemos presentado estudios teóricos y de simulación para una molécula rígida de polielectrolito, inmerso en un electrolito tipo MPR. Hemos encontrado un acuerdo cualitativo excelente entre la teoría HNC/MSA y los datos de DME. Particularmente para el caso en que los parámetros de la molécula de polielectrolito fueron mapeados a los parámetros de la molécula de $\mathrm{ADN}$, encontramos además un acuerdo cuantitativo excelente. El mejor acuerdo cuantitativo en las geometrías plana y esférica, con los datos de IIC reportados en las Refs. $[69,84,101]$, se debe a que las interacciones de corto alcance usadas en $\mathrm{MC}$ son iguales a las del modelo teórico y no solamente similares como en este estudio.

Annque la teoría HNC/MSA ya había sido aplicada al modelo de un polielectrolito inmerso en un electrolito tipo $\operatorname{MPR}[29,80]$, nuestro trabajo está centrado en el estudio del sobrecargado por lo que debemos puntualizar nuestras contribuciones:

- La confirmación del comportamiento no monotónico del potencial $\zeta$ como función de la densidad superficial de carła sobre la varilla.

- El hecho de que se produce sobrecarga en un electrolito al incrementar el tamaño o 
la concentración de los iones y, en principio. sin importar la valencia.

- En un electrolito formado por iones divalestes el sobrecargado desaparece cuando la concentración o el tamaño de los iones disminuye.

El comportamiento no monotónico del potencial $\zeta$ tiene implicaciones en los cálculos de electroforesis. La teoría estándar para la eler troforesis $[108,109]$ está basada en lass predicciones de Poisson-Boltzmann para el fotencial $\zeta$ y por lo tanto, deben encontrarse diferencias importantes si se aplica la teoría HNC/MSA al problema de la electroforesis. En la teoría de Poisson-Boltzmann, el potencial $\zeta$ como una función de la densidac̀ superficial de carga de la varilla es una función monotónica, mientras que en la teoría HNC/MSA no es así. En [29] se sugiere por primera vez que este efecto puede producir un cambio en la dirección de la movilidad electroforética. Esto se debe básicamente al comportamiento no monotónico del potencial $\zeta$. Recientemente, Lozada-Cassou et al. [35] extendieron la teoría de la electroforesis para incluir efectos de tamaño iónico, a través de la teoría HNC/MSA, la cual fue aplicada para macroiones esféricos. Un revertimiento en la dirección de la movilidad fue encontrado además de un comportamiento altamente no lineal de la movilidad, como una función del potencial $\zeta$. Se encontró que estas predicciones están de acuerdo con los resultados experimentales, contrario con la teoría estándar de la electroforesis, en donde la movilidad es universal.

Finalmente, la posibilidad de producir sobrecargado por iones monovalentes mientras que los iones divalentes no siempre lo producen (aún en condiciones de alta concentración) está en contraposición con la creencia general, además de que pone en evidencia la importancia de considerar propiamente los efectos entrópicos (volumen excluido) en la descripción de dicho efecto. Este resultado es particularmente relevante ya que pone en evidencia un mecanismo que no es propiamente tomado en cuenta en las teorías semifenomenológicas que tratan de describir al sobrecargado. Este hecho será fuertemente discutido en el siguiente capitulo. 


\section{Capítulo 3}

\section{Un criterio universal para el sobrecargado}

\subsection{Introducción}

En el capítulo anterior hemos estudiado la sobrecarga de una molécula de ADN. Así mis-. mo, verificamos la existencia de sobrecarga por medio de dinámica molecular. En dicho capítulo evidenciamos que las correlaciones de corto alcance, y las correlaciones por carga juegan un papel importante en el sobrecargado. Así por ejemplo, se ha mostrado que la concentración y el tamaño iónico desempeñan un papel fundamental en dicho efecto. Con el propósito de esclarecer los mecanismos que conducen al sobrecargado, en este capítulo estudiamos la doble capa eléctrica de una superficie plana y cargada, en contacto con un electrolito tipo modelo primitivo restringido simétrico. De esta forma, mostraremos que el sobrecargado puede describirse únicamente en términos de tres parámetros adimensionales, los cuales están relacionados con el volumen excluido de los iones y las energías de interacción ion-ion e ion-pared. Hemos construido un diagrama de sobrecargado en términos de estos parámetros. Bajo este esquema, damos un criterio para el umbral de sobrecargado. Finalmente concluiremos que el sobrecargado es un estado ordenado que se puede producir ya sea, al incrementar el volumen excluido o la energía electrostática de interacción, pero siempre como una combinación de ambos.

\subsection{La doble capa eléctrica $\cdot$}

\subsubsection{La ecuación HNC/MSA para una superficie plana}

De acuerdo con el método propuesto en al capitulo 1, se pueden deducir las ecuaciones. para un fluido en presencia de un campo externo de una manera directa. Las ecuaciones HNC/MSA para un electrolito del tipo modelo primitivo restringido, de $N$ especies, en contacto con una superficie cargada, están dadas por $[63,69]$ 


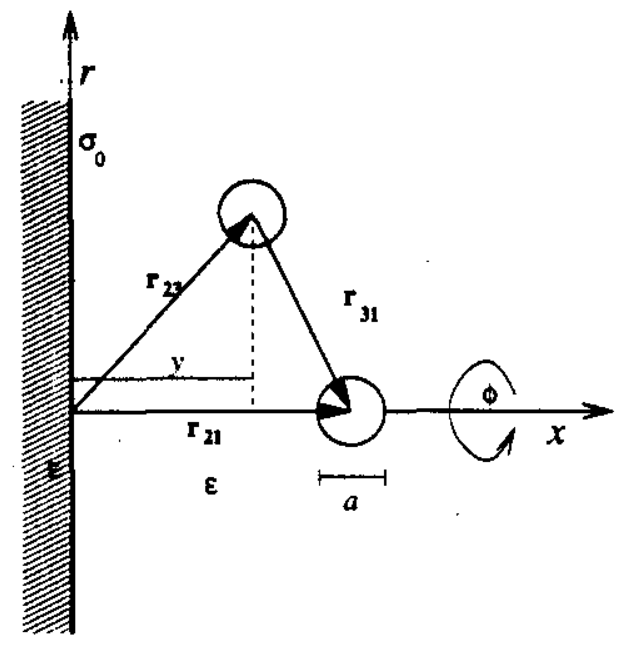

Figura 3.1: Representación esquemática del modelo usado en la derivación de la ecuación HNC/MSA para un electrolito tipo modelo primitivo restringido en contacto con una superficie cargada, plana e infinita.

$$
g_{\alpha i}\left(\mathbf{r}_{21}\right)=\exp \left\{-\beta u_{\alpha i}\left(\mathbf{r}_{21}\right)+\sum_{m=1}^{N} \rho_{m} \int c_{i m}\left(\mathbf{r}_{13}\right) h_{\alpha m}\left(\mathbf{r}_{23}\right) d v_{3}\right\}
$$

donde $u_{\alpha i}\left(\mathbf{r}_{21}\right)$ es el potencial de interacción directa entre las partículas 2 y 1 de la especie $\alpha$ e $i$, respectivamente. Suponiendo en la Ec.(3.1) que, la especie $\alpha$ corresponde a una superficie infinitamente extendida cuya concentración, $\rho_{\alpha}$, tiende a cero, y las otras especies corresponden a un electrolito tipo modelo primitivo restringido con partículas de diámetro $a$, el cual es descrito por la aproximación esférica media. Integrando en coordenadas cilíndricas, la Ec.(3.1) se escribe como

$$
\begin{aligned}
g_{\alpha i}(x)= & \exp \left\{-\beta u_{\alpha i}(x)+2 \pi \int_{-\infty}^{\infty} h_{\alpha s}(y) d y \int_{0}^{\infty} c_{s}(s) r d r\right. \\
& \left.+2 \pi z_{i} \int_{-\infty}^{\infty} h_{\alpha d}(y) d y \int_{0}^{\infty} c_{d}^{s r}(s) r d r-\frac{2 \pi \beta e^{2} z_{i}}{\varepsilon} \int_{-\infty}^{\infty} h_{\alpha d}(y) d y \int_{0}^{\infty} \frac{r d r}{r}\right\},
\end{aligned}
$$

donde $i=1.2, \ldots, N: x y$ y son las distancias perpendiculares a la superficie de los iones 1 y 3 , respectivamente. $s$ es la distancia entre los iones 1 y 3 (ver Fig. 3.1);

$$
\begin{array}{r}
h_{\alpha s}(y)=\sum_{m=1}^{n} \rho_{m} h_{\alpha m}(y) ; \\
h_{\alpha d}(y)=\sum_{m=1}^{n} z_{m} \rho_{m} h_{\alpha m}(y) ; \\
c_{i m}(s)=c_{s}(s)+z_{i} z_{m} c_{d}^{s r}(s)-\beta e^{2} z_{i} z_{m} / \varepsilon s .
\end{array}
$$


Debido al uso de las coordenadas cilíndricas $r \mathrm{~d} r=s \mathrm{~d} s$, de esta forma la Ec.(3.2) se convierte en

$$
\begin{aligned}
g_{\alpha i}(x)= & \exp \left\{-\beta u_{\alpha i}(x)+2 \pi \int_{a / 2}^{\infty} K(x, y) h_{\alpha s}(y) d y+2 \pi \int_{-\infty}^{a / 2} K(x, y) h_{\alpha s}(y) d y\right. \\
& \left.+2 \pi z_{i} \int_{a / 2}^{\infty} L(x, y) h_{\alpha d}(y) d y+\frac{2 \pi \beta e^{2} z_{i}}{\varepsilon} \int_{a / 2}^{\infty}|x \cdots y| h_{\alpha d}(y) d y\right\}
\end{aligned}
$$

en donde hemos definido

$$
\begin{gathered}
K(x, y) \equiv \int_{|x-y|}^{\infty} c_{s}(s) s d s \\
L(x, y) \because \int_{|x-y|}^{\infty} c_{d .}^{s r}(s) s d s
\end{gathered}
$$

El potencial de interacción entre la molécula $\alpha$ y el ion $i$, se puede dividir en un término de esfera dura-pared rígida, $u_{\alpha i}^{*}(x)$, más la contribución electrostática $u_{\alpha i}^{e l}(x)$. La contribución de $u_{\alpha i}^{*}(x)$ es considerada en los límites de integración. De la ley de Gauss, $u_{\alpha i}^{e l}(x)$ está dado por

$$
-\beta u_{\alpha i}^{e l}(x)=\frac{2 \pi \beta e z_{i}}{\varepsilon} \sigma_{0} x
$$

donde $\sigma_{0}$ es la densidad superficial de carga sobre la superficie. Dado que $h_{\alpha i}=-1$ para $x \leq a / 2$, la Ec.(3.3) se puede escribir como

$$
\begin{aligned}
g_{\alpha i}(x)= & \exp \left\{-\beta u_{\alpha i}(x)+2 \pi \int_{a / 2}^{\infty} K(x, y) h_{\alpha s}(y) d y+J(x)\right. \\
& \left.+2 \pi z_{i} \int_{a / 2}^{\infty} L(x, y) h_{\alpha d}(y) d y+\frac{2 \pi \beta e^{2} z_{i}}{\varepsilon} \int_{a / 2}^{\infty}|x-y| h_{\alpha d}(y) d y\right\}
\end{aligned}
$$

donde

$$
J(x)=-2 \pi \rho_{T} \int_{-\infty}^{a / 2} K(x, y) d y
$$

Las expresiones para los kérneles se encue tran dadas en el Apéndice B. La ecuación H.NC MSA. i. e., Ec. (3.5), puede ser escrita de manera conveniente como

$$
g_{i}(x)=\exp \left\{-\beta w_{i}(x)\right\}=\exp \left\{-3 q_{i} \iota^{\prime}(x)+w_{i}^{\mathrm{sr}}(x)\right\} .
$$

Podemos ver que el potencial de la fuerza media ent e a suj,erficie y un ion de la especie $i$ tiene dos contribuciones principales: (i) la contribucín elect rostática dada por el potencial electrostático medio, $\psi(x)$, la cual, para geometría slena e: expresada como

$$
\psi(x)=-\frac{2 \pi}{\varepsilon} \sigma_{0} x-\frac{1}{\varepsilon} \int \frac{\rho_{\mathrm{el}}(y)}{s} d v_{3}=-\frac{2 \pi}{\xi} c_{0} x-\frac{2 \tau}{\xi} \cdot \int_{a / 2}^{\infty} \rho_{\mathrm{el}}(y)|x-y| d y
$$


donde hemos definido $\rho_{\mathrm{el}}(x) \equiv \sum_{i=1}^{n} q_{i} \rho_{i} g_{i}(x)$. (ii) El potencial, $w_{i}^{\mathrm{gr}}$, debido a las correlaciones de corto alcance

$$
w_{i}^{\mathrm{sr}}(x)=2 \pi \int_{a / 2}^{\infty} K(x, y) h_{\alpha s}(y) d y+J(x)+2 \pi z_{i} \int_{a / 2}^{\infty} L(x, y) h_{\alpha d}(y) d y
$$

En el caso de $q_{i}=0, \psi(x)=0 \mathrm{y}$ el fluido es un fluicio de esferas duras en donde solamente los términos de corto alcance son incluidos en la Ec.(3.6). Por otro lado, en el iimite de iones puntuales la Ec.(3.6) se escribe como

$$
g_{i}(x)=\exp \left\{-\beta q_{i} \psi(x)\right\}=\exp \left\{\frac{2 \pi}{\varepsilon} \sigma_{0} \beta q_{i} x+\frac{2 \pi}{\varepsilon} \beta q_{i} \int_{a / 2}^{\infty} \rho_{\mathrm{el}}(y)|x-y| d y\right\}
$$

la cual es la versión integral de la ecuación de Poisson-Boltzmann, dado que $w_{i}^{\mathrm{sr}}(x)=0$ para $a=0$. De esta forma, podemos ver que la teoría de Poisson-Boltzmann debe fallar en sus predicciones cuando las correlaciones de corto alcance se vuelven importantes, esto es: Bajo condiciones de un alto volumen excluido, bajo condiciones de un fuerte acoplamiento ion-ion y/o ion-campo eléctrico. En la teoría de Poisson-Boltzmann la mayor parte del campo eléctrico es apantallado a una distancia igual a la longitud de Debye $\lambda_{\mathrm{D}}=1 / \kappa$, donde $\kappa$ ha sido definida en la Ec.(B.4). El hecho de que algunas correlaciones de largo alcance más la entropía del gas ideal sean consideradas, se ve reflejado en el ancho de la doble capa eléctrica (DCE) predicho por la teoría de Poisson-Boltzmann. La teoria de Poisson-Boltzmann no predice sobrecargado debido a que las correlaciones de corto alcance no han sido incluidas de ninguna forma. Nuestro propósito es mostrar que la sobrecarga ocurre como una combinación de ambos, acoplamiento electrostático y volumen excluido. El sobrecargado puede identificarse al observar los perfiles reducidos de concentración(PRC), $g_{\alpha i}(x)$, el potencial electrostático promedio, $\psi(x)$ o el campo eléctrico local, $E(x)$. El potencial electrostático promedio, dado en la Ec.(3.7), es desde luego la solución de la ecuación de Poisson

$$
\nabla^{2} \psi(x)=\frac{4 \pi e}{\Xi} \rho_{e l}(x) .
$$

Las condiciones de frontera que tienen que satisfacerse para el potencial electrostático medio. $\varphi(x)$. y el campo eléctrico, $E(x)=-\frac{d v \cdot(x)}{d x}$, son

$$
\lim _{x \rightarrow \infty} \psi(x)=0 . \quad \lim _{x \rightarrow \infty} \frac{d \psi(x)}{d x}=0 .
$$

El campo eléctrico total a una distancia dada de la superficie está dado por

$$
E(x)=\frac{2 \pi}{\Xi} \sigma(x) \quad \text { con } \quad \sigma(x)=\sigma_{0}+\sigma^{\prime}(x),
$$


donde $\sigma(x)$ y $\sigma^{\prime}(x)$ son los perfiles de distribución de carga, total e inducida, a una distancia $x$ de la superficie, respectivamente. El perfil de distribución de carga inducida está dado por

$$
\sigma^{\prime}(x)=\int_{a / 2}^{x} \rho_{\mathrm{el}}(x) d x
$$

El cual debe cumplir que $\lim _{x \rightarrow \infty} \sigma^{\prime}(x)=-\sigma_{0}$, para satisfacer la condición de electroneutralidad. Una cantidad útil para identificar la región de sobrecargado puede ser el perfil de carga total, $\sigma(x)$, ya que esta cantidad es la que indica los cambios de signo del carn. po eléctrico. Con el propósito de evaluar la sobrecarga definimos el perfil reclucido de distribución de carga total (PRCT), $P(x)$, de la siguiente manera

$$
P(x) \equiv \frac{\sigma(x)}{\sigma_{0}}=1+\frac{\sigma^{\prime}(x)}{\sigma_{0}},
$$

el cual será mayor que cero en las regiones de sobrecargado.

\subsubsection{Algunos resultados típicos de HNC/MSA y Poisson-Boltzmann}

La Ec.(3.5) puede resolverse con técnicas avanzadas de elemento finito. De la solución de dicha ecuación sè obtienen de manera directa los PRC. En la Fig. (3.2) se muestran dos soluciones típicas de la Ec.(3.5) para un electrolito 1:1, a dos concentraciones diferentes de $\rho=1 \mathrm{M}$ y $\rho=0.1 \mathrm{M}$, en presencia de un campo externo producido por una densidad superficial de carga de $0.1 \mathrm{C} / \mathrm{m}^{2}$. Como es de esperarse, la concentración de los contraiones va disminuyendo a medida que la distancia a la superficie se va incrementando hasta que llega a un valor constante. Por el contrario, la concentración de los coiones cerca de la superficie es muy baja y se va incrementando conforme la distancia a la placa se incrementa, hasta que alcanza un valor constante. Los contraiones son atraídos hacia la superficie, sin embargo, no condensan para cancelar el campo ya que cada uno de ellos posee energía cinética. Conforme se va acumulando carga en la superficie la intensidad del campo va disminuyendo de manera que la contribución de la energía cinética le gana a la energía electrostática de atracción hacia la superficie.

Al disminuir la concentración, el ancho de la DCE tiende a incrementarse. de manera que para $\rho=1.0 \mathrm{M}$ los PRC tienden a un valor constante más cerca de la superficie que para $\rho=0.1 \mathrm{M}$. Para $\rho=1.0 \mathrm{M}$ los PRC alcanzan un valor constante para $x \approx 5.2 a / 2$, mientras que para $\rho=0.1 \mathrm{M}$ éste se alcanza en $x \approx 15.0 a / 2$. El hecho de que la DCE se compacte al incrementar la concentración tiere que ver con que existe menos volumen accesible por cada partícula ${ }^{1}$, lo que hace que las partículas en el bulto tiendan a compactarla. Vamos a referirnos a la región de bulto como aquella donde el fluido es homogéneo, i. e. donde el perfil de concentración es constante. En la Fig. 3.3a se muestra el potencial electrostático promedio, $\psi(x)$, para un electrolito monovalente a una concentración de 1.0.M. Los parámetros son los mismos que en la Fig. 3.2. El comportamiento

\footnotetext{
${ }^{1}$ Como discutimos en el capitulo 1 , esto corresponde a una disminución de la entropía del sistema.
} 


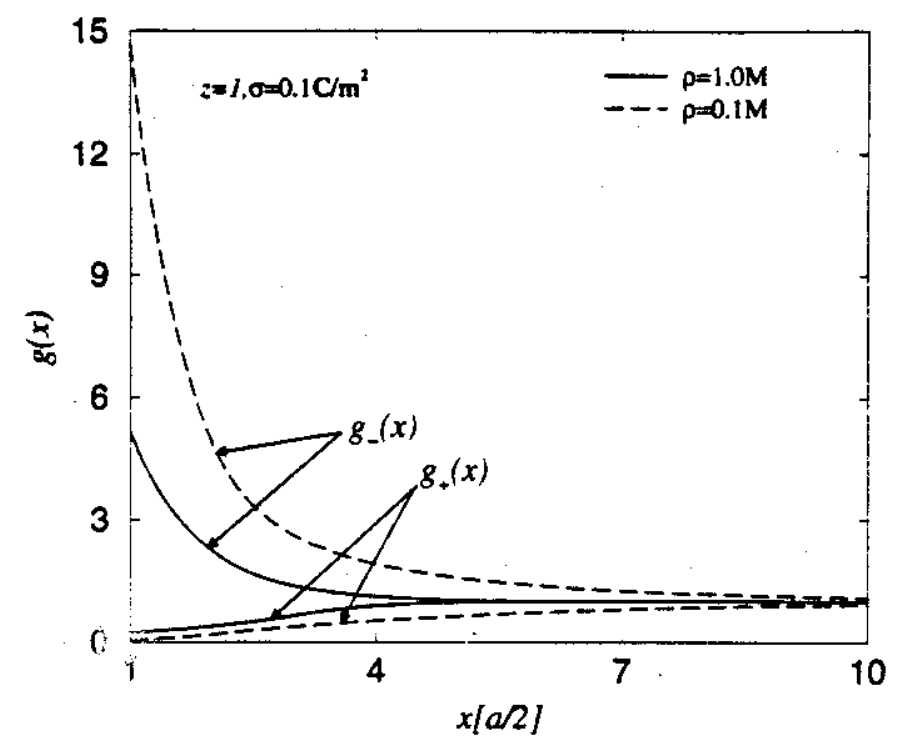

Figura 3.2: PCR obtenidos de HNC/MSA, para un electrolito tipo MPR y monovalente en presencia de un campo eléctrico producido por una densidad de carga superficial de $\sigma_{0}=$ $0.1 \mathrm{C} / \mathrm{m}^{2}$. Los cálculos fueron hechos para $T=298 \mathrm{~K}, \varepsilon=78.5$ y $a=4.25 \AA$. La línea continua representa los PCR para $\rho=1.0 \mathrm{M}$, mientras que la línea discontinua representa los PCR para $\rho=0.1 \mathrm{M}$.
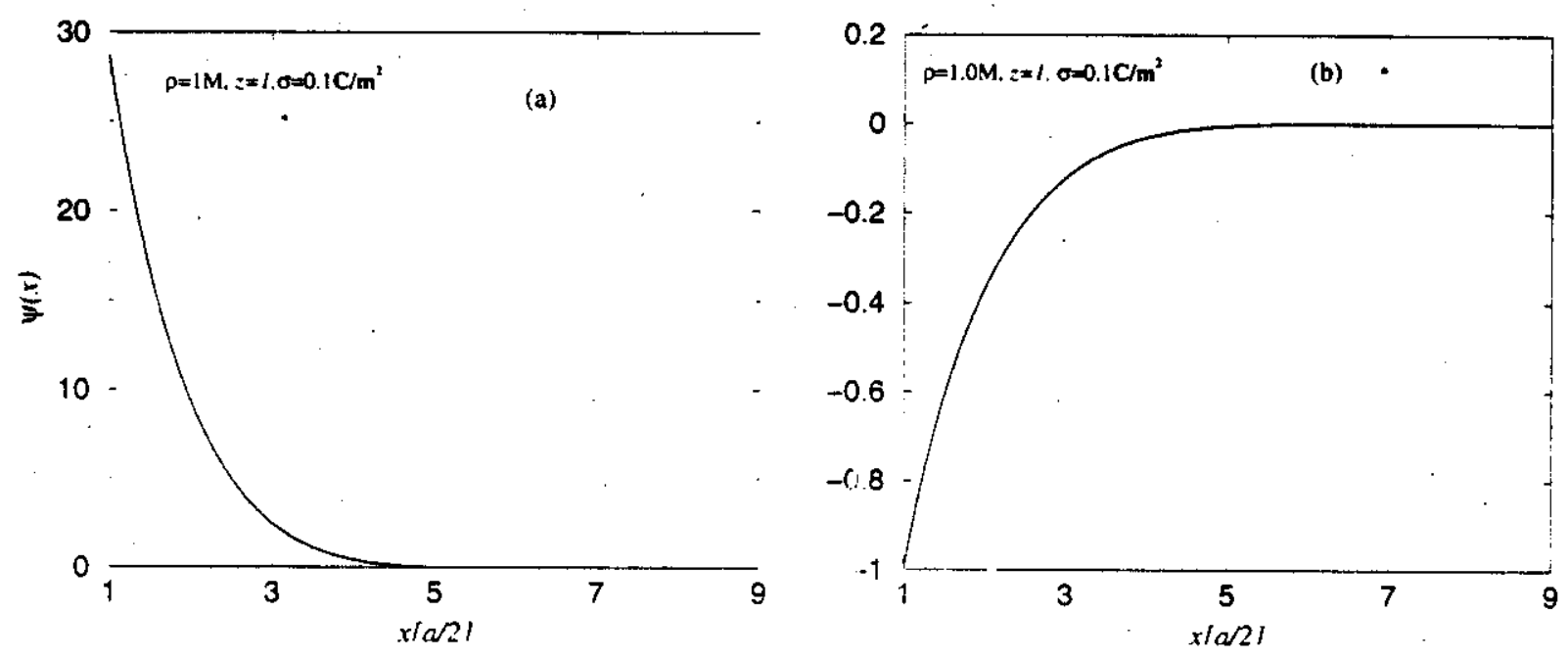

Figura 3.3: (a)Potencial electrostático promedio, $\omega(x)$, y (b) perfil reducido de carga, $P(x)$, para el electrolito de concentración $\rho=1$.JM ce la Fig: 3.2 .

de $\psi(x)$ es monotónico decreciente has a que alcanza el valor constante de $\psi(x)=0$, conforme $x \rightarrow \infty$. En la Fig. 3.3b se muestra ol perfil reducido de carga para el mismo electrolito. Observamos que $P(x)$ tiene un comportaniento monotónicamente creciente 
Figura 3.4: PRC para un electrolitc divalente en presencia de un campo producido por una densidad superficial de carga de $\sigma_{0}=0.1 \mathrm{C} / \mathrm{m}^{2}$. Los cálculos fueron hechos para $T=298 \mathrm{~K}$, $\varepsilon=78.5$ y $a=4.25 \AA$. Las línea:i continua y discontinua representan resultados de HNC/MSA para los PCR de los contraiones y coiones, respectivamente. La línea punteada representa los PCR obtenidos de la solución de la ecuación de Poisson-Boltzmann.

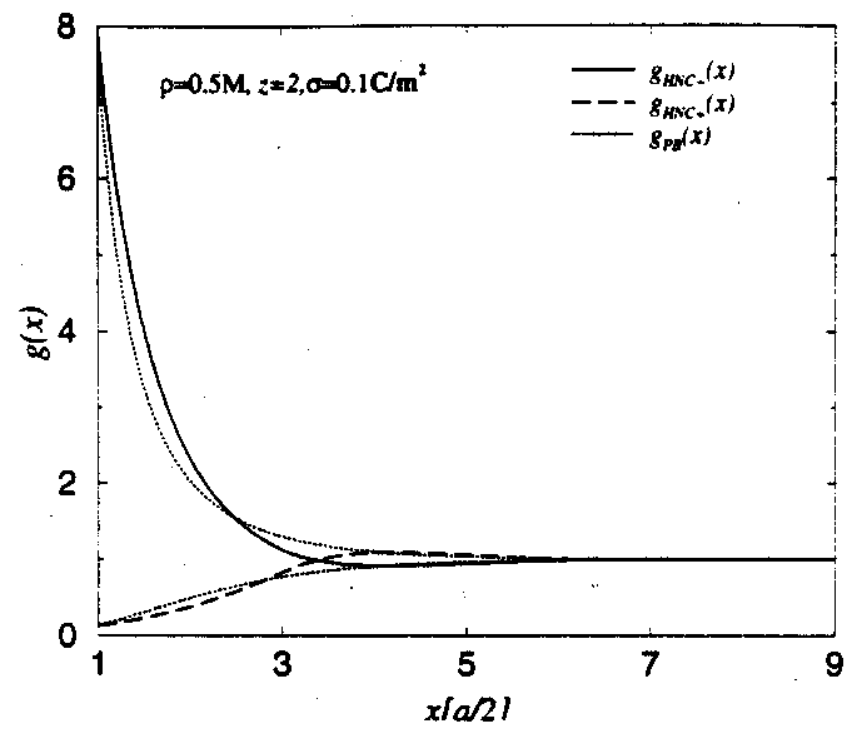

hasta que alcanza el valor de $P(x)=0$. Este comportamiento de las dos funciones, $\psi(x)$ y $P(x)$, es típico en la ausencia de sobrecargado.
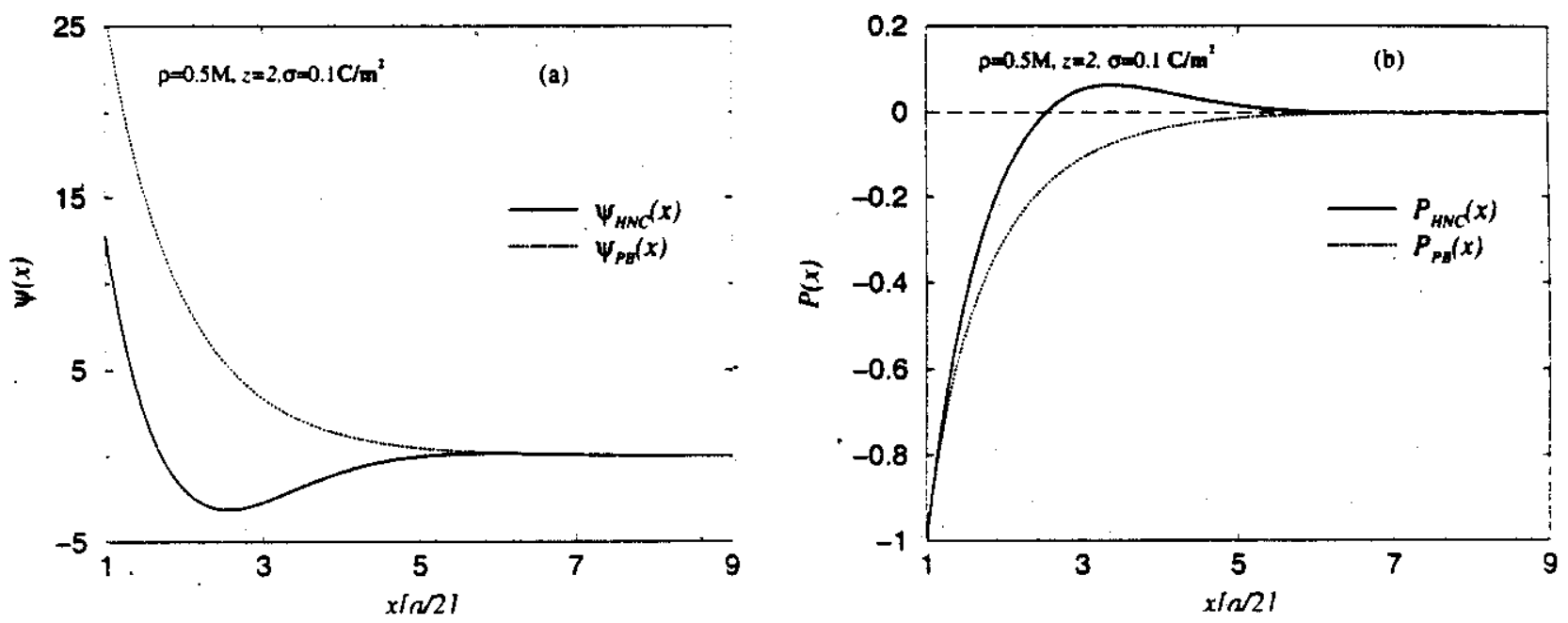

Figura 3.5: (a)Potencial electrostácicico promedio, $\psi(x)$, y (b) perfil reducido de carga, $P(x)$, para un electrolito divalente de conct:ntración $\rho=0.5 \mathrm{M}$. Los parámetros son los mismos que los de la Fig. 3.4. La línea contini a epresenta los resultados de HNC/MSA, mientras que la línea punteada representa los resuitados obtenidos de Poisson-Boltzmann.

En la Fig. (3.4) se muestri n lo PRC para un electrolito 2:2, a una concentración de $\rho=0.5 \mathrm{M}$, en presencia de un c๕moo externo producido por una densidad superficial de carga de $0.1 \mathrm{C} / \mathrm{m}^{2}$. Así como pera el electrolito monovalente, se observa que la concentración de los contraiones va di minı yendo a medida que la distanciś a la superficie se va incrementando, por el contrario, lii concentración de los coiones cerca de la superficie está por debajo de la concentıación d bulto y se va incrementando conforme la distancia 
a la placa aumenta. A diferencia del caso monovalente de la Fig. 3.2, los PRC no muestran un comportamiento monotónico (decreciente para los contraiones o creciente para los coiones), sino que para valores de $x$ entre $x \approx 3.3 a / 2$ y $x \approx 6.3 a / 2$, la concentración de los contraiones muestra valores per debajo de la concentración de bulto, mientras que la concentración de los coiones por encima, i. e., los PRC presentan oscilaciones. Esto indica la formación de una primer t capa cerca de la superficie, predominantemente de contraiones, y posteriormente una segunda capa formada predominantemente por coiones. El comportamiento oscilatorio de los PRC es típico en presencia de sobrecargado. Por el contrario, los PRC obtenidos por medio de la ecuación de Poisson-Boltzmann no muestran este comportamiento sino que mantienen un comportamiento monotónico. Lo que indica que esta teoría no predice sobrecargado.

En las Figs. 3.ja y 3.5b se muestran el potencial electrostático promedio, $\psi(x)$, y el perfil reducido de carga, respectivamente, para un electrolito divalente a una concentración de $0.5 \mathrm{M}$. Los parámetros son los mismos de la Fig. 3.4. En este caso, $\psi(x)$ presenta valores negativos y además se muestra la aparición de un mínimo, lo que indica un cambio de pendiente en $\psi(x)$, por lo tanto un cambio en la dirección del campo eléctrico. Es decir, después de la ocurrencia del mínimo de potencial el campo eléctrico cambia de dirección. En la región donde el campo eléctrico invierte su dirección es donde se ocurre la sobrecarga. El perfil reducido de carga (Fig. 3.5b) presenta un máximo, lo que indica la presencia de sobrecargado. En la región de sobrecargado $P(x)>1$. Esto indica que el fluido se acomoda de forma que la carga sobre la superficie no solamente es cancelada sino que en esta región además es sobrecompensada. La localización, $x_{0}$, del mínimo de potencial, $\psi_{\text {min }}$, corresponde exactamente al punto donde la gráfica de la función $P(x)$ corta por primera vez el punto de $P(x)=1$. En este caso $\psi_{\min }$ está localizado en $x_{0}=2.5 a / 2$ y la función $P(x=2 . \bar{a} a / 2)=1$. La función $P(x)$ sigue siendo creciente para $x_{0}<x \leq x_{1}$, siendo $x_{1}$ la localización del máximo de la función $P(x), P_{\max }$. Esto implica que los contraiones se siguen atrayendo hacia la superficie, a pesar de que el campo eléctrico ha sido cancelado e incluso ha cambiado de dirección. En esta región el término dominante de la interacción contraión-superficie es el término $w_{i}^{\mathrm{sr}}(x)$, de la Ec.(3.6); el cual es atractivo. El punto $x_{1}$ corresponde exactamenie a el punto donde se cumple que $g_{+}=g_{-}$, es decir el punto donde las oscilaciones de los PPC empiezan. Los resultados para $P(x)$ y $\psi(x)$, obtenidos de la ecuación de Poisson-Buitzmann preservar un comportamiento monotónico como era de esperarse, ya que esta teo ía no predice sobrecargado, de manera consistente, dado que en esta teoría no se incluye d término $u_{i}^{\mathrm{sr}}(x)$.

En la Fig. 3.6 se muestra las, sclu ic nes de las ecuaciones HNC/MSA y de PoissonBoltzmann, Ecs. (3.5) y (3.9), respectivar nente, para un electrolito $1: 1$ a una concentración de $\rho=1 \mathrm{M}$, en presencia de un carnpo cléctrico producido por una densidad superficial de carga de $0.1 \mathrm{C} / \mathrm{m}^{2}$. En este casc lis sálcuios fueron hechos para $a=7.4 \hat{A}$. Observamos que el hecho de aumentar el tamaño de los iones se ve reflejado en la aparición de oscilaciones en los PRC predichos por la teoria HNC/MSA. Por otro lado, se favorece la adsorción de ambas especies en la suf erfici s. respecto a las curvas correspondientes a los 


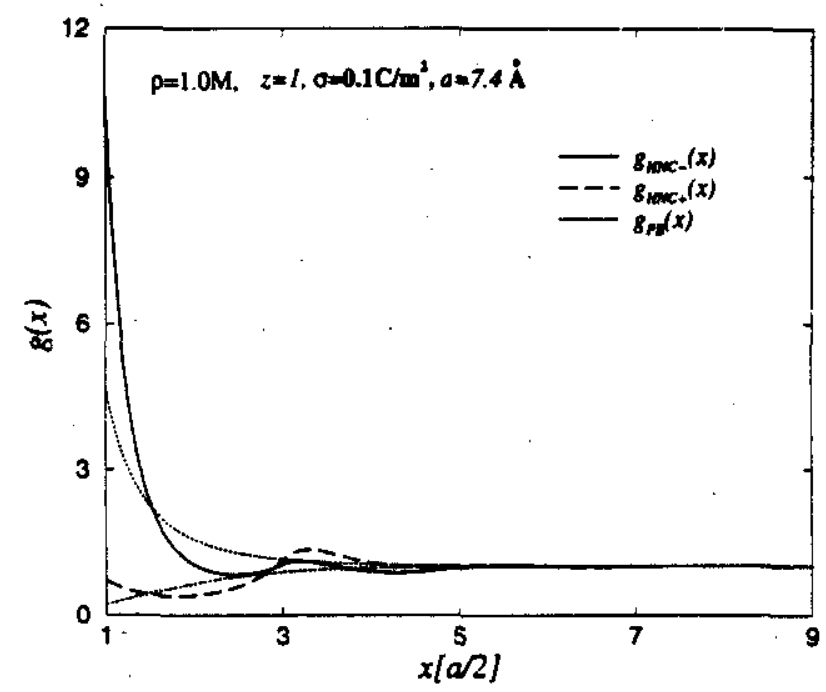

Figura 3.6: PRC para un electrolito monovalente en presencia de un campo producido por una densidad de carga de $\sigma_{0}=0.1 \mathrm{C} / \mathrm{m}^{2}$ sobre la superficie. Los cálculos fueron hechos para $T=298 \mathrm{~K}, \varepsilon=78.5$ y $a=7.4 \AA$. Las lineas continua y discontinua representan resultados de HNC/MSA para los PRC de los contraiones y coiones, respectivamente. La línea punteada representa los resultados de Poisson-Boltzmann para los PCR.

mismos parámetros de la Fig. $3.2^{2}$. Este hecho puede notarse en el incremento de los valores de contacto de los PRC, i. e., $g_{i}(x=a / 2)$. La aparición de las oscilaciones en los PRC tiene que ver directamente con el sobrecargado, como ya se mencionó anteriormente. Sin embargo, a diferencia de los PRC del electrolito divalente, donde también ocurre sobrecargado, en este caso la localización del máximo del PRC para los coiones no coincide con el mínimo del PRC para los contraiones, sino que los máximos de ambas funciones están muy próximos entre si, lo cual quiere decir que la aparición de éstos se debe, principalmente, a los efectos de tamaño y en un grado menor a las correlaciones electrostáticas. Los PRC obtenidos de la ecuación de Poisson-Boltzmann son cualitativamente similares a los mostrados para el caso concentrado de la Fig. 3.2. Este resultado no debe sorprendernos ya que en los resultados de la Fig. 3.2 las correlaciones por volumen excluido nos son muy importantes, mientras que en este caso no son consideradas.

La verificación del sobrecargado se lleva a cabo observando la gráfica de las funciones $\psi(x)$ y $P(x)$, las cuales se presentan el las Figs. 3.7. Los parámetros son los mismos de la Fig. 3.6. Así como en la Fig. 3.5, el potencial electrostático promedio, $\psi(x)$, presenta valores negativos además de la presencia de un mínimo y un máximo, lo que indica dos cambios de signo en la pendiente de $\psi(x)$, y por lo tanto dos cambios en la dirección del campo eléctrico. En este caso $\psi_{\min }$ está localizado en $x_{0}=2.5 \mathrm{a} / 2 \mathrm{y}$ la función

\footnotetext{
${ }^{2}$ Nótesè que el valor de contacto para $g_{i}(x=a / 2)$ de los coiones se incrementa y es diferente de cero tanto para PB como para HNC/MSA. Esto refieja el hecho de que la repulsión entre la placa y los iones disminuye simplemente al incrementar el tamaño. Sin embargo, el valor de $g_{i}(x=a / 2)$ es ligeramente mayor para HNC/MSA que para PB, lo cual se debe a la inclusión de las correlaciones por tamaño.
} 

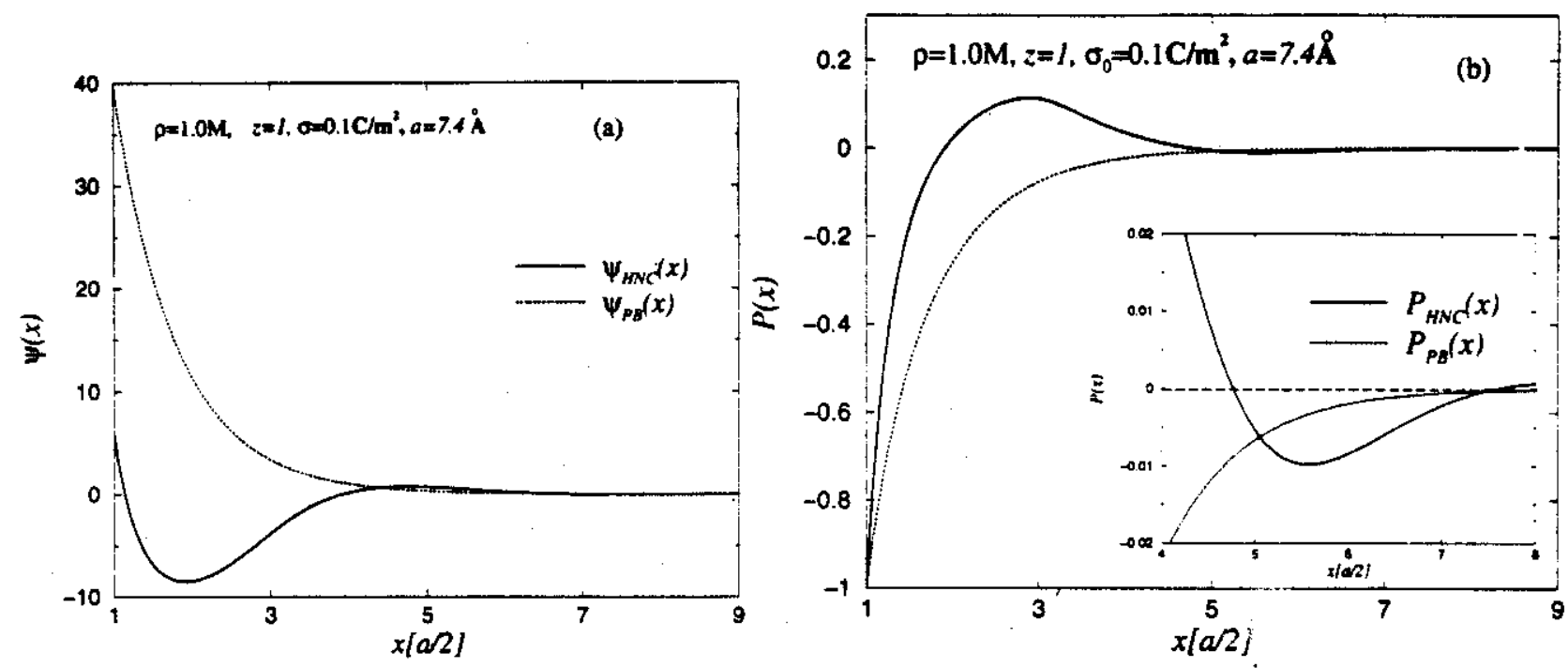

Figura 3.7: (a)Potencial electrostático promedio, $\psi(x)$, y (b) perfil reducido de carga, $P(x)$, para los mismos parámetros de la Fig. 3.6. Las líneas continuas representan los resultados de HNC/MSA, mientras que la línea punteada representa los datos de Poisson-Boltzmann.

$P(x=2.5 a / 2)=1$. L La presencia de un máximo en el perfil reducido de carga indica un nuevo cambio de dirección en el sentido del campo eléctrico. Este hecho se refleja en el mínimo que presenta la función $P(x)$, el cual se muéstra en el recuadro de la Fig. 3.7b. Los resultados obtenidos de la ecuación de Poisson-Boltzmann no muestran ningún rasgo característico novedoso.

\subsection{Parámetros adimensionales de la ecuación HNC/MSA}

En la sección anterior hemos presentado algunas soluciones típicas de la ecuación HNC/MSA para un electrolito tipo MPR en presencia de un campo eléctrico producido por una superficie plana y cargada uniformemente, en donde, en algunos de los casos encontramos sobrecarga. De esta forma, mostramos que la sobrecarga puede ocurrir para iones monovalentes. Hasta ahora, no se han precisado los mecanismos que conducen a la aparición de dicho efecto, en donde es claro que concentración y tamaño iónico juegan un papel importante y similar, pero aun no establecido. En la siguiente sección vamos a discutir algunos de estos mecanismos en donde se aclara de que forma se desempeñan, ambos parámetros. concentración y tamaño.

Partiendo de la Ec.(3.5), podemos definir los siguientes parámetros en dicha ecuación:

$$
\xi \equiv \frac{2 x}{a}, \quad \zeta \equiv \frac{q^{2} \beta}{a \varepsilon}, \quad \gamma_{i} \equiv \frac{2 \pi 3}{\varepsilon} q_{i} \sigma_{\ni} a, \quad y \quad \eta \equiv \frac{\pi}{6} \rho_{T} a^{3} ;
$$

donde $q_{i}=z_{i}$ e y $q=\left|z_{i}\right| e$. El parámetro $\xi$ mide la distancia perpendicular a la superficie $\mathrm{en}$ 
unidades de radios iónicos, el parámetro $\zeta$ es una medida de la interacción entre dos iones del fluido, el parámetro $\gamma$ mide la interacción ion-superficie y por último, el parámetro $\eta$ mide el volumen excluido por los iones. Partiendo de estas definiciones el potencial electrostático de interacción superficie-ion de la Ec.(3.4) puede reescribirse como

$$
-\beta u_{\alpha i}(\xi) \equiv \frac{\gamma_{i}}{2} \xi
$$

Tomando en cuenta que la función de correlación directa entre dos partículas del fluido depende de su distancia relativa y los parámetros $\zeta$ y $\eta$, i. e., $c_{m j}(s)=c_{m j}(s ; \zeta, \eta)$, encontramos que la ecuación para los PRC puede escribirse como

$$
g_{i}\left(\xi ; \zeta, \gamma_{i}, \eta\right)=\exp \left\{\gamma_{i} \frac{\xi}{2}+\sum_{j=1}^{n} \eta_{j} \int h_{j}\left(\xi^{\prime}(s ; \zeta, \eta) d \mu_{3}\right\},\right.
$$

donde $d \mu_{3}=\frac{6 d v_{3}}{\pi a^{3}}$ y $\eta_{j}=\frac{\pi}{6} \rho_{j} a^{3}$. De esta forma la Ec.(3.5) puede expresarse como

$$
\begin{aligned}
g_{i}\left(\xi ; \zeta, \gamma_{i}, \eta\right)= & \exp \left\{\gamma_{i} \frac{\xi}{2}+\int_{1}^{\infty} \hat{h}_{\alpha d}\left(\xi^{\prime}\right) \hat{K}\left(\xi, \xi^{\prime}\right) d \xi^{\prime}+\hat{J}(\xi)\right. \\
& \left.\hat{z}_{i} \zeta \int_{1}^{\infty} \hat{h}_{\alpha s}\left(\xi^{\prime}\right) \hat{L}\left(\xi, \xi^{\prime}\right) d \xi^{\prime}+3 \hat{z}_{i} \zeta \int_{1}^{\infty} \hat{h}_{\alpha s}\left(\xi^{\prime}\right)\left|\xi-\xi^{\prime}\right| d \xi^{\prime}\right\}
\end{aligned}
$$

en donde $\hat{z}_{j} \equiv q_{j} / q$ y hemos definido

$$
\begin{gathered}
\hat{h}_{\alpha s}\left(\xi^{\prime}\right)=\sum_{m=1 \cdot}^{n} \eta_{m} h_{\alpha m}\left(\xi^{\prime}\right) \\
\hat{h}_{\alpha d}\left(\xi^{\prime}\right)=\sum_{m=1}^{n} \hat{z}_{m} \eta_{m} h_{\alpha m}\left(\xi^{\prime}\right) .
\end{gathered}
$$

Las expresiones para $\hat{K}\left(\xi, \xi^{\prime}\right), \hat{L}\left(\xi, \xi^{\prime}\right)$ y $\hat{J}(\xi)$ están dadas a continuación. Para $\xi-2 \leq$ $\xi^{\prime} \leq \xi+2$,

$$
\hat{K}\left(\xi, \xi^{\prime}\right)=\frac{3}{4} \gamma_{1}\left[\left(\xi-\xi^{\prime}\right)^{2}-4\right]+\frac{3}{2} \eta \gamma_{2}\left[\left|\xi-\xi^{\prime}\right|^{3}-8\right]+\frac{3}{160} \eta \gamma_{1}\left[\left|\xi-\xi^{\prime}\right|^{5}-32\right]
$$

y

$$
\hat{L}\left(\xi, \xi^{\prime}\right)=3\left[2-\left|\xi-\xi^{\prime}\right|\right]-\frac{3}{2} \frac{\Gamma a}{(1+\Gamma a)}\left[4-\left(\xi-\xi^{\prime}\right)^{2}\right]+\frac{1}{4}\left(\frac{\Gamma a}{1+\Gamma a}\right)^{2}\left[8-\left(\xi-\xi^{\prime}\right)^{3}\right]
$$

La expresión para $J(x)$ se puede obtener analíticamente

$$
\hat{J}(\xi)=\frac{1}{4} \eta \gamma_{1}\left[\hat{p}^{3}-12 \hat{p}+16\right]+\frac{3}{8} \eta^{2} \gamma_{2}\left[\hat{p}^{4}-32 \hat{p}+48\right]+\frac{1}{320} \eta^{2} \gamma_{1}\left[\hat{p}^{6}-192 \hat{p}+320\right]
$$


para $1 \leq \xi \leq 3$ y cero de cualquier otra forma, hemos definido $\hat{p} \equiv \xi-1$. De esta forma podemos ver que podemos estudiar los perfiles de concentración en términos de tres parámetros adimensionales, dado que la función $g_{i}(\xi)$ depende exclusivamente de $\zeta$, $\gamma, \eta$ y $\xi$; independientemente de la combinación de los valores de $q, \sigma_{0}, T, \varepsilon, \rho$ y $a$. 


\subsection{Resultados}

El sobrecargado ocurre en la región en $x$ donde $\sigma^{\prime}(x)$ sobrecompensa a $\sigma_{0}$, i. e., el campo eléctrico invierte su dirección. Con el propósito de evaluar la sobrecarga, definimos la condición de sobrecargado como $\chi \equiv \frac{\sigma_{\max }^{\prime}}{\sigma_{0}}>1$, siendo $\sigma_{\max }^{\prime}$ el valor máximo de $\sigma^{\prime}(x)$ en el intervalo $x \in[a / 2, \infty)$. Lado que estamos tratando con electrolitos simétricos $\rho \equiv \rho_{1}=\rho_{2}$. Como ya mostramos anteriormente, las funciones de distribución dependen únicamente de $\eta, \zeta$ y $\gamma$.

La descripción en términos de estos parámetros es de utilidad ya que elimina un nûmero considerable de variables $\left(q, \sigma_{0}, \varepsilon, T, \rho\right.$ y $\left.a\right)$. Por otro lado, da una cuantifiçación de las diferentes contribuciones físicas del sistema. En nuestros resultados vamos a dar únicamente los valores de los parámetros adimensionales.

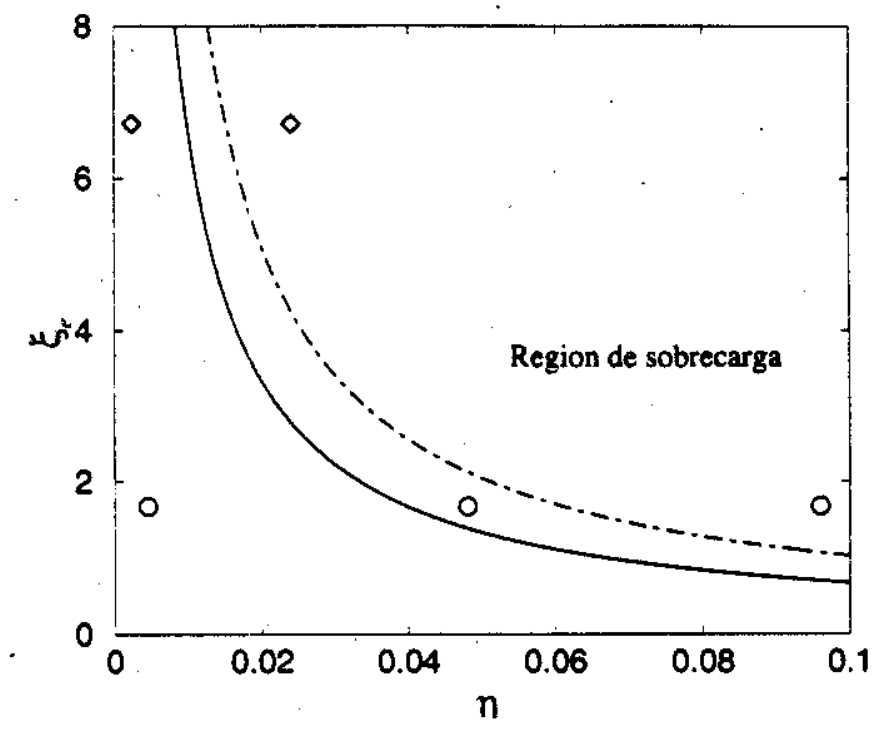

Figura 3.8: Diagrama de sobrecargado en el plano $\zeta-\eta$. Por encima de la línea continua se cumple $\chi>1.000001$, mientras que la línea discontinua es para $\chi>1.01$. Los puntos marcados con diamantes abiertos son para un electrolito $2: 2$ a concentración $0.5 \mathrm{M}$ (izquierda) y $0.5 \mathrm{M}$ (derecha). Los círculos en blanco son para un electrolito $1: 1$, a concentración de $0.1 \mathrm{M}$ (izquierda), $1 \mathrm{M}$ (centro) y $2.0 \mathrm{M}$ (derecha). Para estos puntos $a=4.25 \AA, T=298 \mathrm{~K}, \varepsilon=78.5$.

Hemos resuelto numéricamente la Ec.(3.15) y mapeado, en un plano $\xi_{e}-\eta$, la línea límite sobre la cual se satisface la condición de sobrecarga. Por encima de la línea continua la pared está sobrecargada mientras que, por debajo el sobrecargado no ocurre. Para dar una idea de las condicienes bajo las cuales se presenta el sobrecargado, en la Fig. 3.8 presentamos la localización de algunas soluciones electrolíticas típicas. Para los propósitos de la generación de la línea límite de sobrecargado hemos tomado la condición de sobrecargado ${ }^{3} \chi>1.000001$. La línea discontinua fue generada considerando $\chi>1.01$

\footnotetext{
${ }^{3}$ Esta condición es arbitraria y es usarla únicamente por razónes computacioneles.
} 
y podemos notar que hay una gran diferencia entre ambas curvas, sin embargo, la última puede ser tomada como una buena propuesta por razones de resolución numérica en métodos en donde no es posible tener tal resolución, por ejemplo, en cálculos de simulación molecular. Al ajustar la curva de sobrecargado con una función encontramos que se cumple, de manera universal;

$$
\eta \xi_{e}=0.066
$$

Dada la definición de la longitud de Debve

$$
\lambda_{D}=1 / \kappa \quad \text { con } \quad \kappa^{2}=\frac{4 \pi \beta}{\epsilon} \sum_{i=1}^{N} q_{i}^{2} \rho_{i}
$$

puede escribirse

$$
\eta \xi_{e}=\frac{1}{24} \kappa^{2} a^{2}=0.066
$$

Esta condición se satisface independientemente de la intensidad del campo así como de la geometría. De esta forma, el criterio para la ocurrencia de sobrecargado i. e., $\equiv \frac{1}{24} \kappa^{2} a^{2}>$ 0.066 es univers a l.

Se observa que el sobrecargado puede ocurrir bajo condiciones de bajo volumen excluido (valores pequeños de $\dot{\eta}$ ), pero iones fuertemente acoplados (valores altos de $\zeta$ ), (punto B). De otra manera puede ocurrir para iones débilmente acoplados en un sistema con un volumen excluido relativamente alto. El eje $\eta$ representa el límite del fluido de esferas duras mientras que el eje $\zeta$ representa el límite de cero volumen excluido, que es el límite de iones puntuales. En ambos casos límite el sobrecargado no se presenta. Todas las líneas límite de sobrecargado son asintóticas a los ejes $\eta$ y $\zeta$, independientemente del valor del parámetro $\gamma$. Para que el sobrecargado se presente ambos parámetros, $\eta$ y $\zeta$ deben ser diferentes de cero. Mientras que el sobrecargado no se presenta en ausencia de carga, la adsorción de ambas especies de iones se encuentra regulada puramente por el efecto de volumen excluido $y$ ambas especies son adsorbidas de manera simétrica. De esta forma, un minimo en la carga de la superficie es suficiente para romper esa simetría $\mathbf{y}$, por lo tanto, ocurre sobrecargado aun para densidades de carga muy bajas. Lo sorprendente aquí es que para que empiece la sobrecarga no depende de la magnitud del campo sino que es una propiedad del electrolito.

En las Figs. 3.9 mostramos los perfiles reducidos de carga total, $P(x)$, para distintos valores del parámetro de volumen excluido $\eta$. manteniendo constante $\zeta$ y $\gamma$. En la Fig. 3.9a se toman los valores de $\zeta=6.7$ y $y=2.4$. mientras que en la Fig. $3.9 \mathrm{~b}$ se toman los valores de $\zeta=6.7 y=2.4 \times 10^{-3}$. El PRCT es el mismo para cualquier combinación de los parámetros del fluido siempre que $\zeta, \gamma \mathrm{y} \eta$ permanezcan constantes. Como un ejemplo, las curvas sólidas de las Figs. 3.9, pueden ser generadas con alguna de las combinaciones de parámetros dados en la Tabla 3.1. Las primeras dos combinaciones corresponden a la curva sólida de la Fig. 3.9a mientras que las dos últimas a la curva sólida de la Fig. 3.9b. De acuerdo con la definición del parámetro de volumen excluido, para mantener $\eta$ constante, 

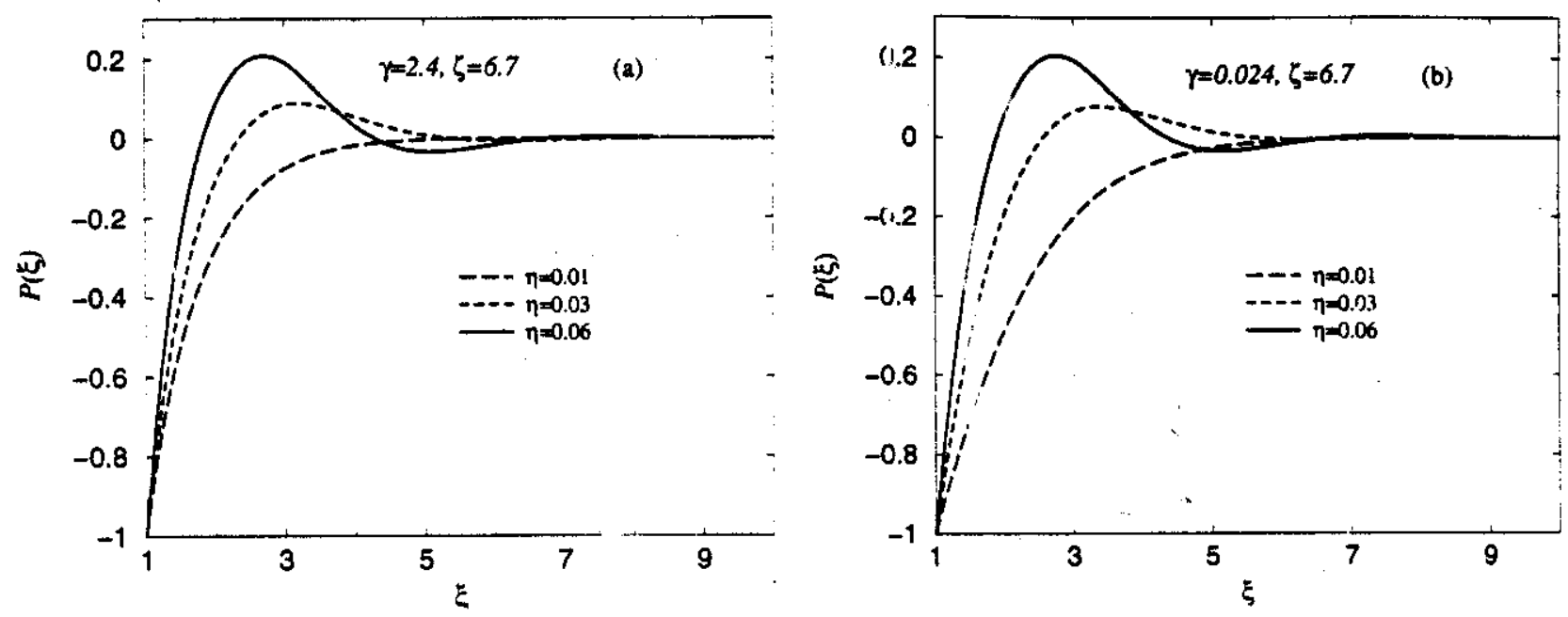

Figura 3.9: PRACT, $P(\xi)$, como una función de $\xi$, para (a) $\xi_{e}=6.7$ y $\gamma=2.4$ y (b) $\xi_{e}=6.7$ y $\gamma=2.4 \times 10^{-3}$. En cada caso se muestran los resultados para $\eta=0.01,0.03$ y 0.06 .

si el diámetro iónico, $a$, se incrementa por un factor, digamos $\nu$, la concentración del electrolito debe reducirse por un factor adecuado para mantener $\eta$ constante. Por otro lado, para mantener $\gamma$ constante, debe suceder que el campo eléctrico efectivo producido por la superficie, $E_{0}=\frac{2 \pi \sigma_{0}}{\varepsilon}$, disminuya por un factor $\nu$ o la temperatura aumente por el mismo factor. Esto significa por ejemplo, que si las curvas mostradas en las Figs. 3.9, hubieran sido generadas incrementando el diámétro de las partículas, la carga superficial correspondiente a cada curva debería disminuir conforme se incrementa $\eta$. A pesar de ello se observa que el sobrecargado siempre aumenta conforme aumenta $\eta$. Entre otras cosas, esto implica que no hay un mínimo necesario de $\sigma_{0}$ para tener sobrecargado. Lo cual está en oposición con las predicciones de teorias fenomenológicas[110]. De las Figs. 3.9 es claro que cuanto mavor es el volumen excluido, mavor es el sobrecargado. El efecto es el mismo si se incrementa $\rho$ o $a$, dado que $P(\xi)$ es una función que depende únicamente de $\eta, \zeta$ y $\gamma$

Tabla 3.1: Parámetros del sistema con el cual han sido generadas las curvas continuas de la Fig. 3.9.

\begin{tabular}{|c|c|c|c|c|c|}
\hline$z$ & $\sigma_{0}\left[\mathrm{C} / \mathrm{m}^{2}\right]$ & $a \mid-A]$ & $\rho[M]$ & $=$ & $\hat{i}$ \\
\hline 2 & 0.100 & 4.25 & 1.24 & 73.5 & 2.40 \\
\hline 2 & 0.046 & 6.25 & 0.33 & 53.4 & 2.40 \\
\hline 2 & $10^{-3}$ & 4.25 & 1.24 & 78.5 & $2.4 \times 10^{-2}$ \\
\hline 2 & $4.6 \times 10^{-4}$ & 6.25 & 0.39 & 53.8 & $2.4 \times 10^{-2}$ \\
\hline
\end{tabular}

El hecho de incrementar el sobrecargado iacreme itando el volumen excluido es un efecto puramente entröpico. Desde un punto de vis:a mecanístico, esto tiene que ver 
con una fuerza neta hacia la pared sobre una partícula cercana a la superficie, ejercida por todas las partículas restantes, la cual es la misma tanto para los coiones como para los contraiones. Sin embargo, debido al campo eléctrico producido por la superficie, los contraiones son predominantemente adsorbidos y de esta forma el sobrecargado puede aparecer. De esta forma, podemos proponer que el sobrecargado es un estado producido por la disminución de la entropía del sistema. Por lo tanto, los resultados que mostramos aquí implican que este estado puede alcanzarse ya sea, incrementando el volumen excluido o incrementando el acoplamiento eléctrico entre los iones.

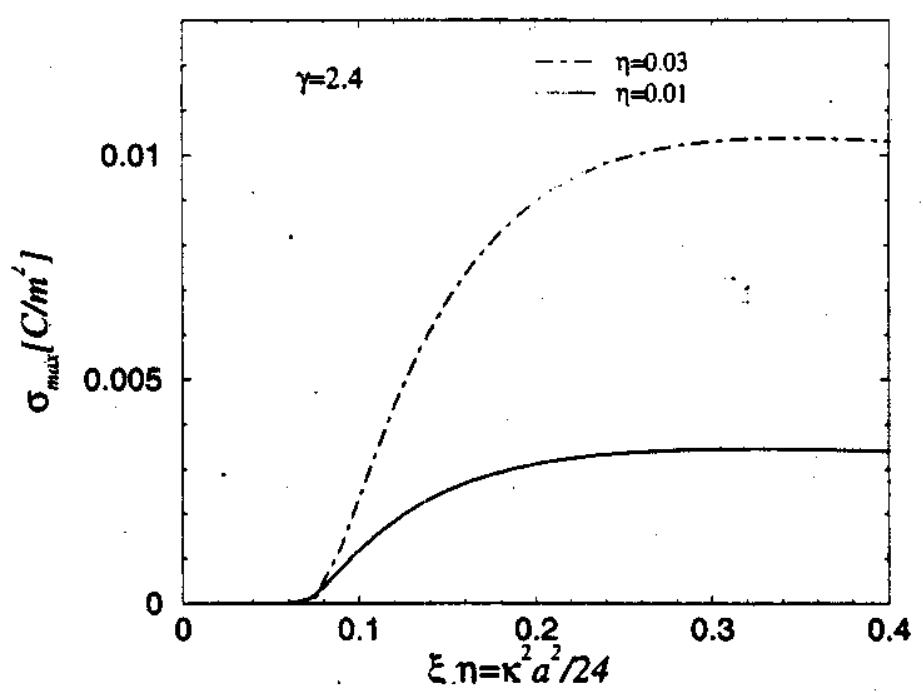

Figura 3.10: $\sigma_{\max }$, en función de $\xi_{e}$, para $\gamma=2.4$. La línea continua corresponde a $\eta=0.01$, mientras que la línea discontinua es para $\eta=0.03$.

El impacto de los parámetros adimensionales $\eta$ y $\zeta$ se analiza en la Fig. 3.4, donde $\sigma_{\max } \equiv \max \{\sigma(x)\}$ (ver la Ec.(3.11)) es graficada como una función de $\xi_{e} \zeta$, para dos valores diferentes de $\eta$. En ambos casos el valor del parámetro $\gamma$ se mantiene constante. Observamos que el sobrecargado es mavor en el caso de $\eta=0.03$, para todos los valores de $\zeta$. Esto era de esperarse ya que un valor mavor en $\eta$ implica menor volumen accesible, por lo tanto menor entropía y mayor adsorción. Es notable el hecho de que la sobrecarga empieza en el mismo valor de $\xi_{e} \zeta=0.066$, sin embargo conforme este punto se suepera el sobrecargado aumenta de manera diferente dependiendo del valor de $\eta$.

El potencial electrostático reducido (PER),

$$
\hat{\psi}(\xi) \equiv q \beta \psi(x),
$$

es también una cantidad que depende únicamente de los parárietros $\eta, \zeta, \gamma$ y $\xi$. Debido a que el valor del potencial electrostático medio en la capa de deslizamiento es importante en los experimentos de electroforesis, nos vemos obligados a analizar la dependencia de esta función en términos de los parámetros adimensionales. Definimos $\hat{\psi}_{0}$, como el potencial 

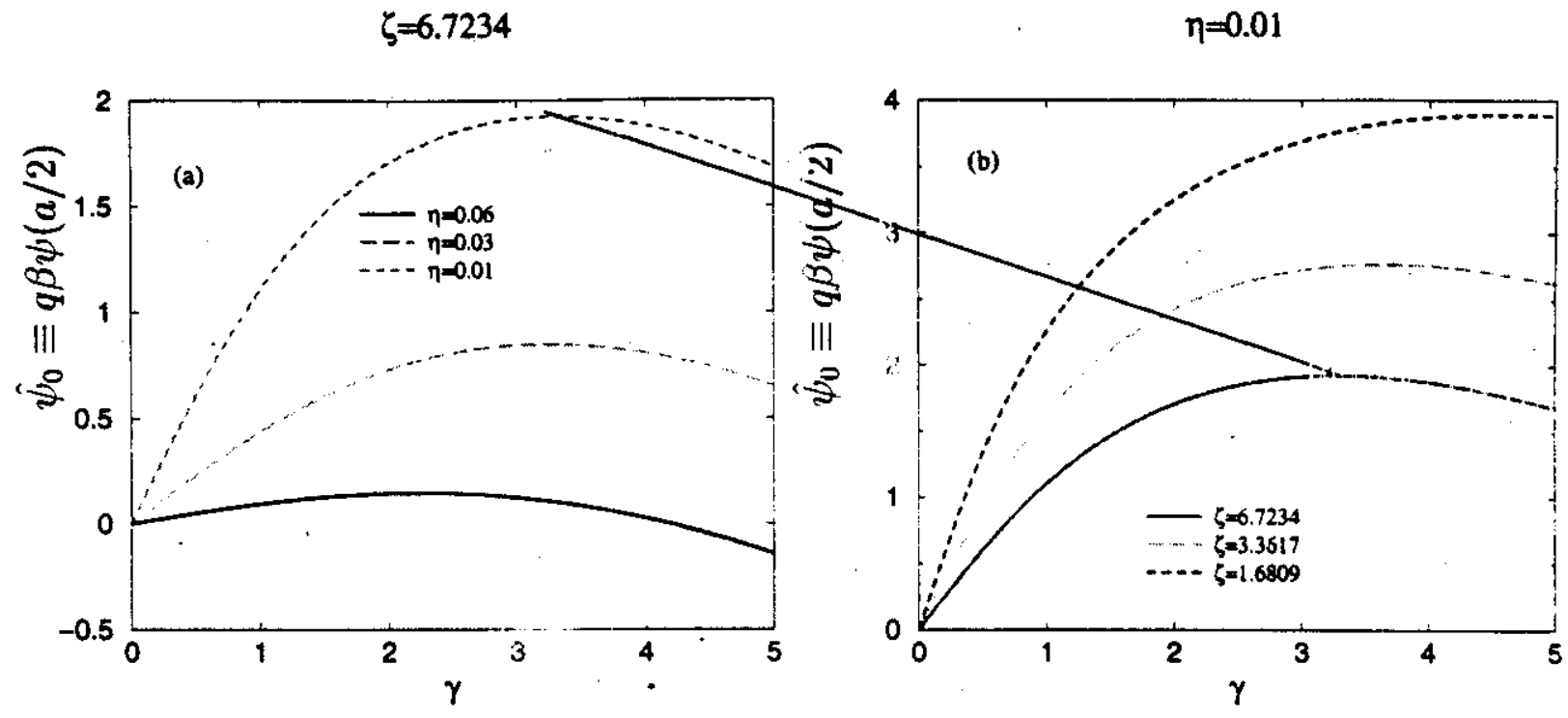

Figura 3.11: Potencial electrostático reducido (PER) en el contacto, $\hat{\psi}_{0}$, como una función del parámetro $\gamma$.(a) resultados para tres valores diferentes del parámetro $\eta$, manteniendo constante el parámetro $\zeta=6.72$, (b) resultados para tres valores diferentes de $\zeta$, manteniendo constante el parámetro $\eta=0.01$. La flecha marca la correspondencia entre ambás curvas.

electrostático reducido en el contacto, i. e.,

$$
\hat{\psi}_{0} \equiv \hat{\psi}(1)=q \beta \psi(a / 2)
$$

En la Fig. 3.11, se muestra el PER de contacto, $\hat{\psi}_{0}$, como una función del parámetro $\gamma$. En la Fig. 3.11a se analiza el efecto del volumen excluido sobre esta función. Observamos que cada curva alcanza un máximo y posteriormente decrece. Por otro lado, un aumento en el volumen excluido, $\eta$, tiene el efecto de disininuir el valor de $\hat{\psi}_{0}$, para un mismo valor de $\gamma$. En este rango de valores de $\gamma$, el valor de $\hat{\psi}_{0}$ puede volverse incluso negativo, como es el caso de $\eta=0.06$ (línea continua). Es inportante remarcar que para este valor del parámetro $\zeta$, en los casos mostrados li: f inción siempre presenta un máximo, el cual desaparece al disminuir el valor de dicho pirámetro. Este hecho se ilustra en la Fig. 3.11b, donde se presenta un análisis de la funciór l l'o para distintos valores de $\zeta$ y manteniendo $\eta=0.01$. Podemos observar que el máximı de la función va desapareciendo gradualmente conforme el valor del parámetro $\zeta$ va dis ninuyelido. Debemos puntualizar el hecho do que un incremento de ambos, volumen excluidr y acoplarniento electrostático, conduce a una disminución del valor del PER en el c.snar to. Sin embargo, la aparición del máximo está relacionado únicamente con un increr ae.ıto e $1 \zeta$. 


\subsection{Conclusiones de capítulo}

En nuestra comparación entre HNC/MSA con los resultados de Poisson-Boltzmann se muestra que la teoría de PB no predice la ocurrencia de sobrecargado. Esto se debe esencialmente a que PB ignora completamente las correlaciones de corto alcance. Por otro lado al incluirlas (aunque sea de manera parcial en HNC/MSA) puede producirse sobrecarga. De esta forma podemos concluir que el sobrecargado resulta de una combinación de efectos entrópicos y de correlaciones electrostáticas.

De este capítulo debemos destacar los siguientes resultados:

- El hecho de que el sobrecargado (así como las funciones $\psi, P(x) g(x)$, etc.) pueden ser descritos en términos de tres parámetros adimensionales. La importancia de este resultado reside, por ejemplo, en el hecho de que un electrolito polivalente puede mapearse en un electrolito monovalente $o$ un electrolito concentrado puede mapearse en un electrolito diluido.

- Los parámetros que describen al sobrecargado son una combinación de ambos efectos, entropía y energía. De esta forma es posible ver que los parámetros dominantes son, la interacción ion-ion y el volumen excluido; dado que se encuentra que el sobrecargado ocurre aun para campos eléctricos muy débiles.

- El poder describir al sobrecargado en términos de estos parámetros provee del criterio para la ocurrencia de dicho efecto, y así de manera $u n i v$ e $r$ s a l ocurre sobrecargado para $\xi_{e} \eta>0.066$.

A principio de este capítulo mencionamos que ambos, concentración y tamaño iónico, desempeñan un papel importante en la ocurrencia de sobrecargado. Es un hecho que a mayor $a$ o $\rho$ (tamaño y concentración), el volumen accesible del sistema es menor. De esta forma, de acuerdo a nuestra discusión en la sección 1.1, a menor volumen accesible del sistema la entropía del sistema es menor. Por lo tanto, a mayor tamaño o concentración de los iones menor entropía del sistema. Las correlaciones por carga entre partículas igualmente cargadas también tienen el efecto de disminuir la entropía del sistema. Por lo tanto. podemos concluir que el sobrecargado aparece como una consecuencia de la disminución de la entropía del sistema. Lo cual puede llevarse a cabo incrementando el tamaño. la concentración y/o la magnitud de la interacción entre los iones. Es importante remarcar que el efecto de volumen excluido está incluido en el potencial de corto alcance, $u_{i}^{\text {sr }}$, de la Ec. (3.6) y que es éste el responsable de la atracción de los contraiones hacia la pared en la región de sobrecargado. Esto es, en la región de sobrecargado la atracción debida a las correlaciones es mayor que la repulsión eléctrica en esa misma región. De esta forma, la inclusión de las correlaciones de corto alcance se vuelve necesaria para describir correctamente al sistema. Esto es, la teoría de Poisson-Boltzmann no describe corréctamente al sistema debido a que las correlaciones de corto alcance no son tomadas en cuenta. 
En nuestros resultados es posible ver que el sobrecargado puede alcanzarse ya sea, al incrementar el volumen excluido o al incrementar el acoplamiento coulómbico. Anteriormente habiamos mostrado que la sobrecarga puede alcanzarse al incrementar el volumen excluido del sistema [111]. Por otro lado, a temperatura cero se demuestra que el efecto de sobrecargado está presente como un efecto puramente electrostático [23, 110]. Nuestros resultados son cor.sistentes con ambos hechos. 


\section{Capítulo 4}

\section{Adsorción de macroiones}

\subsection{Introducción}

La formación de nanoestructuras a partir de la adsorción en solución, se ha convertido en un método muy exitoso dentro de la ingeniería molecular. En los últimos años, este método ha permitido la fabricación de estructuras bien organizadas de moléculas orgánicas relativamente complejas, por ejemplo, cadenas largas de ácidos grasos, tioles e incluso de polímeros de alto peso molecular. La aplicación de esta técnica para la formación de nanoestructuras de moléculas más simples, tales como iones inorgánicos, ha demostrado ser mucho más difícil. Un intento por crear una técnica que permita la formación de tales nanoestructuras, consiste en formar primero una estructura autoensamblada, Ia cual es usada como 'patrón' para controlar la adsorción de los iones inorgánicos. De esta forma, ha sido posible crear películas organizadas de varios iones, por la adsorción en solución sobre una monocapa de surfactante insoluble, esparcido sobre la interfase líquido-aire [112].

Para iones monovalentes pequeños y bajas densidades superficiales de carga, la ecuación de Poisson-Boltzmann predice una capa difusa, de espesor $\lambda_{\mathfrak{D}}$. Los experimentos para estos sistemas muestran un excclente acuerdo con la teoría $[113,114]$. Sin embargo, es ampliamente reconocido que par a el caso de macroiones polivalentes, la descripción de estos sistemas tiene desviaciories marcarlas de la descripción clásica ${ }^{1}$. Además de que no existen actualmente datos expetimentales claros ni tampoco modelos teóricos en la literatura que describan correctan€nte esta situación [115].

El sobrecargado aparece $\mathrm{c}(\mathrm{m})$ : $n$ aspecto importante a considerarse en la adsorción

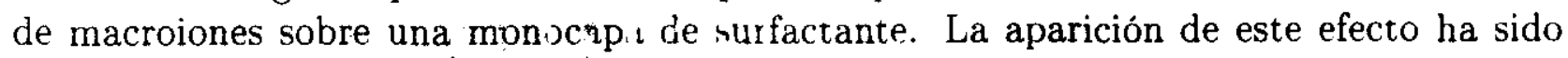
reportada en experimentos recientis it partir de dos observables directos: (1) el potencial electrostático sobre la no:a :apa y (2) el número de contraiones adsorbidos sobre la monocapa. En el primer caso, el fotencial electrostático sobre la monocapa en función de la densidad superficial de car za, presenta una inversión en la polaridad a densidades

\footnotetext{
${ }^{1}$ Por descripción clásica nós referin os a li descripción por medio la ecuación de Poisson-Boltzmann.
} 
superficiales altas. En el segundo caso, se encuentra que para densidades superficiales de carga relativamente altas, el número de contraiones adsorbidos es tal que la carga neta de los contraiones sobrecompensa la carga sobre la superficie [115].

La descripción de estos sistemas a través de la teoría de Poisson-Boltzmann puede conducir a uria descripción no realista del sistema, debido a la no inclusión de correlaciones por tamaño, las cuales son importantes en la aparición de ciertos efectos como ya ha sido discutido previamente en este trabajo (ver Secs. 1.6 y 3.2) [21]. Una apróximación al problema de macroiones en solución, consiste en promediar los grados de libertad de cos microiones de la solución y representarlos mediante el uso de potenciales efectivos [iij]. De esta forma, uno se puede enfocar únicamente a la descripción de los macroiones. La desventaja que presenta este tratamiento es que el uso de parámetros efectivos puede ocultar mucha de la física del problema.

En este capítulo vamos a extender la teoría de ecuaciones integrales HNC/MSA a una solución de macroiones en un electrolito, en contacto con una superficie plana cargada. A través de esta teoría, vamos a tratar de esclarecer los mecanismos de adsorción de macroiones en una superficie. Debido a la inclusión del tamaño de los macroiones en nuestro modelo, esperamos que este modelo sea más realista para describir algunas situaciones experimentales.

\subsection{Modelo}

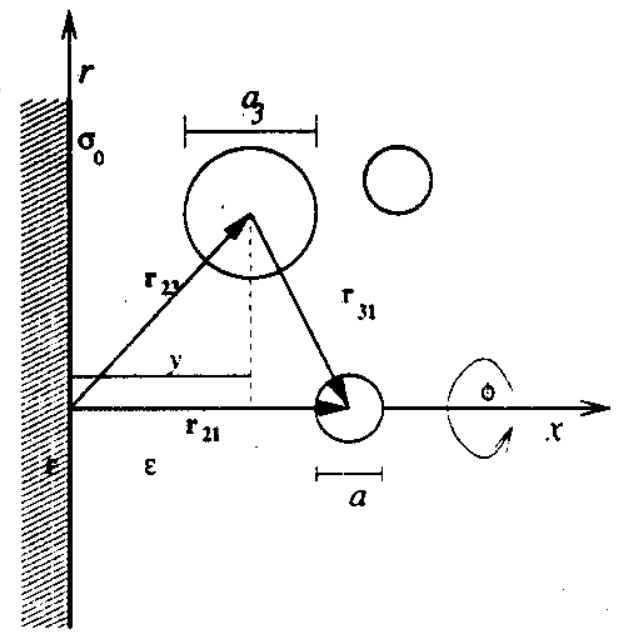

Figura 4.1: Modelo de un fluido de macroiones, tipo modelo primitivo, en contacto con una superficie uniformemente cargada con una densidad superficial de carga $\sigma_{0}$

En este capítulo vamos a considerar una superficie plana en contacto con una solución electrolítica que contiene macroiones más un electrolito tipo modelo primitivo restringido. 


\subsection{La Ecuación HNC/MSA}

La superficie es modelada como una pared infinitamente gruesa con una densidad superficial de carga,$\sigma_{0}$, uniformemente distribuida sobre su superficie. La constante dieléctrica de la superficie es igual a la constante dieléctrica del solvente, para evitar cargas imagen. El fluido con el cual está en contacto la superficie es considerado de la siguiente forma: Es un electrolito de tres especies con dos de ellas, digamos, las especies 1 y 2 , del mismo tamaño y una tercera especie de diferente tamaño, i. e.,

$$
a_{1}=a_{2} \equiv a \leq a_{3}
$$

donde $a_{i}$ es el diámetro de la especie $i$. De aquí en adelante nos vamos a referir a la especie 3 como el macroión. En la Fig. 4.1 se muestra un diagrama del modelo para la superficie y el fluido. Para satisfacer la condición necesaria de campo eléctrico igual a cero lejos de la superficie, la carga sobre la superficie es compensada por un exceso de carga en el fluido, $\sigma^{\prime}$ :

$$
\sigma^{\prime} \equiv \int_{\frac{d+a}{2}}^{\infty} \rho_{e l}(x) d x
$$

donde $\rho_{e l}(x) \equiv \sum_{m=1}^{3} q_{m} \rho_{m}(x)$, siendo $\rho_{m}(x)$ y $q_{m}$ la concentración local y la carga de la especie $m$, respectivamente.

En geometría plana la función de distribución inhomogénea depende solamente de la distancia perpendicular a la placa, así como para los iones esféricos, la función de correlación directa depende únicamente de su distancia relativa. Podemos definir $x=\left|\mathbf{r}_{21}\right|$, $y=\left|\mathbf{r}_{23}\right|$ y $s=\left|\mathbf{r}_{31}\right|$ (ver Fig. 4.1). La interacción entre la superficie y las especies que forman el fluido se lleva a cabo de dos formas diferentes: $(i)$ La interacción por volumen excluido y (ii) la interacción electrostática. De esta forma, el potencial de interacción electrostătica $u_{\alpha i}(x)$, entre la superficie y la $i$-éima especie puede ser escrito como

$$
u_{\alpha i}(x)=u_{\alpha i}^{e l}(x)+u_{\alpha i}^{*}(x)
$$

donde $u_{\alpha i}^{e l}(x)$ es el potencial eléctrico y $u_{\alpha i}^{*}(x)$ es el potencial de inreracción rígida. La interacción rigida entre la superficie y los iones está dada por

$$
u_{\alpha i}^{*}(x)=\left\{\begin{array}{ccc}
\infty & \text { para } & x<\frac{a_{i}}{2} \\
0 & \text { para } & x \geq \frac{a_{i}}{2}
\end{array}\right.
$$

El potencial de interacción electrostática puede encontrarse a partir de la ley de Gauss

$$
-\beta u_{\alpha j}^{e t}(x)=\left\{\frac{2 \pi}{\varepsilon} z_{j} e \beta\left(\sigma_{1}+\sigma_{2}\right) x \text { para } \quad x \geq 0\right.
$$

\subsection{La Ecuación HNC/MSA}

De acuerdo con la sección 1.5 , la ecuación HNC/MSA puede ser deducida usando la aproximación $\mathrm{HNC}$ para la función de correlación directa entre la superficie $y$ las especies 
del fluido y la aproximación MSA entre las componentes del electrolito. Así, la ecuación HNC/MSA está dada por

$$
g_{\alpha i}(x)=\exp \left\{-\beta u_{\alpha i}(x)+\sum_{j=1}^{3} \rho_{i} \int_{0}^{\infty} h_{\alpha j}(y) c_{j i}(s) d v_{3}\right\}
$$

La ventaja de usar MSA para el electrolito es que se tienen resultados analíticos para la función de correlación directa, tanto para el modelo primitivo como para el modelo primitivo restringido. En la aproximación MSA la función de correlación directa para un electrolito tipo modelo primitivo tiene una expresión analítica. Esta función puede ser escrita como

$$
c_{m j}(s)=-\beta u_{m j}^{e l}(s)+c_{m j}^{s r}(s)+c_{m j}^{h s}(s) .
$$

En el primer término aparece el potencial de interacción directa entre dos iones, el cual está dado por la Ec. (1.25). El segundo término es una función de corto alcance y el tercer término es la función de correlación directa para un fluido de esferas duras en la aproximación de Percus-Yevick. Tomando ventaja de la geometría cilíndrica y el hecho de que la función de correlación directa entre los iones depende solamente de sus distancias relativas $s \equiv\left|\mathbf{r}_{1}-\mathbf{r}_{3}\right|$, la Ec. (4.6) puede ser escrita como

$$
\begin{aligned}
g_{\alpha i}(r)= & \exp \left\{\frac{2 \pi}{\varepsilon} z_{i} e \beta \sigma_{0} x-2 \pi A_{i}(x)+2 \pi \sum_{j=1}^{3} \rho_{j} \int_{\frac{a_{j}}{2}}^{\infty} h_{\alpha j}(y)\left(K_{j i}(x, y)+L_{j i}(x, y)\right) d y\right. \\
& \left.+2 \pi z_{i} \frac{e^{2} \beta}{\varepsilon} \sum_{j=1}^{3} z_{j} \rho_{j} \int_{\frac{a_{j}}{2}}^{\infty} g_{\alpha j}(y)|x-y| d y\right\}
\end{aligned}
$$

donde se ha definido

$$
\begin{aligned}
& L_{j i}(r, y)=\int_{|x-y|}^{\infty} c_{j i}^{s r}(s) s d s \\
& K_{j i}(r, y)=\int_{|x-y|}^{\infty} c_{j i}^{h s}(s) s d s
\end{aligned}
$$

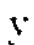

$$
A_{i}(x)=z_{i} z_{3} \rho_{3} \frac{\beta e^{2}}{\varepsilon} \int_{\frac{d+a}{2}}^{\frac{d+a_{3}}{3}}|x-y| d y+\sum_{j=1}^{3} \rho_{j} \int_{-\frac{a_{j}}{2}}^{\frac{a_{3}}{2}}\left(K_{j i}(x, y)+L_{j i}(x, y)\right) d y,
$$

Laas expresiones para los kérneles están dadas en el apéndice C. La ecuación HNC/MSA, i. e., Ec. (4.8), son resueltas numéricamente con técnicas avanzadas de elemento finito. De la solución de la Ec. (4.8), se obtiene la distribución iónica como una función de la distancia a la pared. Ésta nos da la estructura de equilibrio de la DCE, y ésta es expresada en términos de los perfiles reducidos de concentración, $\rho_{\alpha i}(x)=\rho_{i} g_{\alpha i}(x)$. $\rho_{i}$ es la concentración de bulto de la especie $i$, y $g_{\alpha i}(x)$ es el perfil de concentración reducido de la especie $i$. 
De acuerdo con la Ec. (1.12) la ecuación HNC/MSA para los PRC puede escribirse comọ

$$
g_{\alpha i}(x) \equiv \exp \left[-\beta W_{i}(x)\right]=\exp \left[-\beta\left(e z_{i} \psi(x)+J_{i}(x)\right)\right] .
$$

Donde, de acuerdo con la Ec. (4.8), se han identificado dos contribuciones al potencial de la fuerza promedio efectiva pared-ion, $W_{i}(x)$ : la contribución electrostática, dada por: el potencial electrostático promedio, $\psi(x)$, más el potencial repulsivo de corto alcance, debido al tamaño de los iones, el cual está dado por $J_{i}(x)$. Ambas funciones son suncionales de $\rho_{i}(x)$. En la Ec. (4.8) se puede hacer la siguiente identificación.

$$
\begin{aligned}
-\beta J_{i}(x) & =-2 \pi A_{i}(x)+2 \pi \sum_{j=1}^{3} \rho_{j} \int_{\frac{a_{j}}{2}}^{\infty} h_{\alpha j}(y)\left(K_{j i}(x, y)+L_{j i}(x, y)\right) d y \\
-\beta e z_{i} \psi(x) & =\frac{2 \pi}{\varepsilon} z_{i} e \beta \sigma_{0} x+2 \pi z_{i} \frac{e^{2} \beta}{\varepsilon} \sum_{j=1}^{3} z_{j} \rho_{j} \int_{\frac{a_{j}}{2}}^{\infty} g_{\alpha j}(y)|x-y| d y
\end{aligned}
$$

De acuerdo con la ecuación (1.25) la interacción directa ion-ion y macroión-ion están dadas por los potenciales de esfera dura más el potencial Coulómbico. En el caso límite de $a=0$, la ecuación HNC/MSA se reduce a una versión extendida de la ecuación inhomogénea de Poisson-Boltzmann(PB) $[34,117,118]$ para iones puntuales más macroiones, próximos a una pared cargada. Dado que los macroiones son considerados a concentración finita, esta propuestá es una mejora de la ecuación clásica de Poisson-Boltzmann, para macroiones confinạdos a dilución infinita $[34,118]$, donde únicamente dos macroiones son tomados en cuenta: i. e., en nuestra teoría las correlaciones de corto alcance entre los macroiones son incluịdas. Desde luego que para $a=0$ y $\rho_{M}=0$, recuperamos la versión integral de la teoría clásica de Guy-Chapman (GC), para iones puntuales en presencia de una pared cargada [118].

Vamos a denotar como $Z_{M}, a_{M}, \rho_{M}$ a la valencia, diámetro y concentración de los macroiones, respectivamente. De la misma forma, vamos a denotar a los parámetros correspondientes con los subíndices $+\mathrm{y}-$ para los iones positivos y negativos, respectivamente. Hemos resuelto la ecuación HNC/MSA, Ec. (4.8), para varios valores de los parámetros $Z_{M}, a_{M}, \rho_{M}, \sigma_{0}$ y los parámetros del electrolito: $z_{+}: z_{-}, \rho_{i}$ y $a$. Nuestra discusión la vamos a llevar en términos de $g_{\alpha i}(x), \psi(x)$ y la densidad de carga efectiva, la cual está dada por $[10,13]$

$$
\sigma(x)=-\int_{x}^{\infty} \rho_{e l}(y) d y,
$$

donde $\rho_{e l}(x) \equiv \sum_{m=1}^{3} e z_{m} \rho_{m} g_{m}(x)$ es el perfil de carga en la solución. Para satisfacer la condición de electroneutralidad debe cumplirse que

$$
\sigma_{0}=-\int_{a / 2}^{\infty} \rho_{e l}(y) d y .
$$


Por otro lado

$$
\sigma^{\prime}(x) \equiv \int_{a / 2}^{x} \rho_{e l}(y) d y
$$

es la carga inducida por la pared en el fluido, entre la pared y la distancia $x$ a la pared. De aquí que,

$$
\sigma(x) \equiv \sigma^{\prime}(x)+\sigma_{0},
$$

es la carga efectiva, pared más fluido, a una distancia $x$ de la superficie, la cual es una medida directa del sobrecargado. La fuerza electrostática sobre un ion es

$$
f_{i}^{e}(x) \equiv-e z_{i} \frac{\partial \psi(x)}{\partial x}=\frac{4 \pi e z_{i}}{\varepsilon} \sigma(x) .
$$

De esta forma, $\sigma(x)$, no solamente mide el sobrecargado sino que también mide la fuerza efectiva de interacción pared-partícula, la cual está dada por

$$
F_{i}(x)=-\frac{\partial W_{i}(x)}{\partial x}=\frac{1}{\beta} \frac{\partial \ln \left[g_{i}(x)\right]}{\partial x},
$$

la cual es la fuerza efectiva entre la pared y un ion de la especie $i$. De aquí que

$$
F_{i}(x)=f_{i}^{e}(x)+f_{i}^{s}(x),
$$

donde

$$
f_{i}^{s}(x) \equiv \frac{\partial J_{i}(x)}{\partial x}
$$

contiene la contribución no electrostática de la fuerza. Cuanto más grande es el tamaño iónico mavor es el volumen excluido de confinamiento, por lo tanto, menor es el volumen accesible. En nuestro caso, $f_{i}^{s}(x)$ contiene las contribuciones por volumen excluido. Dado que ambos, $\psi(x)$ y $J_{i}(x)$, son funcionales de $\rho_{e l}(y)$ y dado que se encuentran en una ecuación no lineal, las correlaciones por carga $y$ por tamaño son, en general. no independientes $[15,99]{ }^{2}$

\subsection{Resultados}

En todos nuestros cálculos hemos tomado $\mathrm{T}=298 \mathrm{~K}, \varepsilon=78.5 \mathrm{y} \rho_{M}=0.01 \mathrm{M}$. El diámetro de los iones pequeños lo hemos considerado $a=4.25 \mathrm{~A}$, el cual corresponde al tamaño de un ion hidratado, aproximadamente. En la Fig. 4.2, los macroiones tienen carga negativa,

\footnotetext{
${ }^{2}$ Como ya discutimos en la sección 1.1 es un resultado de la mecánica estadística que cuanto menor es el volumen accesible del sistema, menor es la entropía del sistema. Por lo tanto, cuanto más grandes sean los iones menor será la entropia del sistema. Las correlaciones por carga entre partículas igualmente cargadas, producen el efecto también de reducir el volumen accesible del sistema y, por lo tanto, de reducir su entropía.
} 
la cual es opuesta en signo a la de la pared y a la del ion divalente del electrolito. El PRC del macroión muestra una fuerte adsorción hacia la pared. Una segunda capa de macroiones es adsorbida, mediada por una capa de iones positivos divalentes, seguidos por una capa de iones monovalentes negativos. Los macroiones de la primera capa son rodeados por sus contraiones. Capas subsecuentes de macroiones menos concentrados son observadas, mediadas también por capas de iones positivos y negativos. Tomando en cuenta que la concentración de bulto de los macroiones es de $0.01 \mathrm{M}$, la concentración local de los macroiones en el segundo pico es de $\approx 0.035 M$, la cual no es de ninguna manera despreciable. La concentración en el primer pico es de $\approx 20 M$. Por lo que podemos decir que macroiones altamente cargados se ensamblan sobre la superficie. En el recuadro de la Fig. 4.2, a una distancia de $x=a_{3} / 2$, un mínimo muy pronunciado es observado, el cual corresponde a un fuerte revertimiento de la carga (RC El máximo localizado alrededor de $x=15 a / 2$ muestra inversión de la carga (IC). Para : $\leqslant a_{3} / 2$, se presenta el verdadero sobrecargado de la pared: Esto es, los coiones divalentes de la pared son adsorbidos y su carga excede la de la pared más la de los iones negativos pequeños. Este efecto no

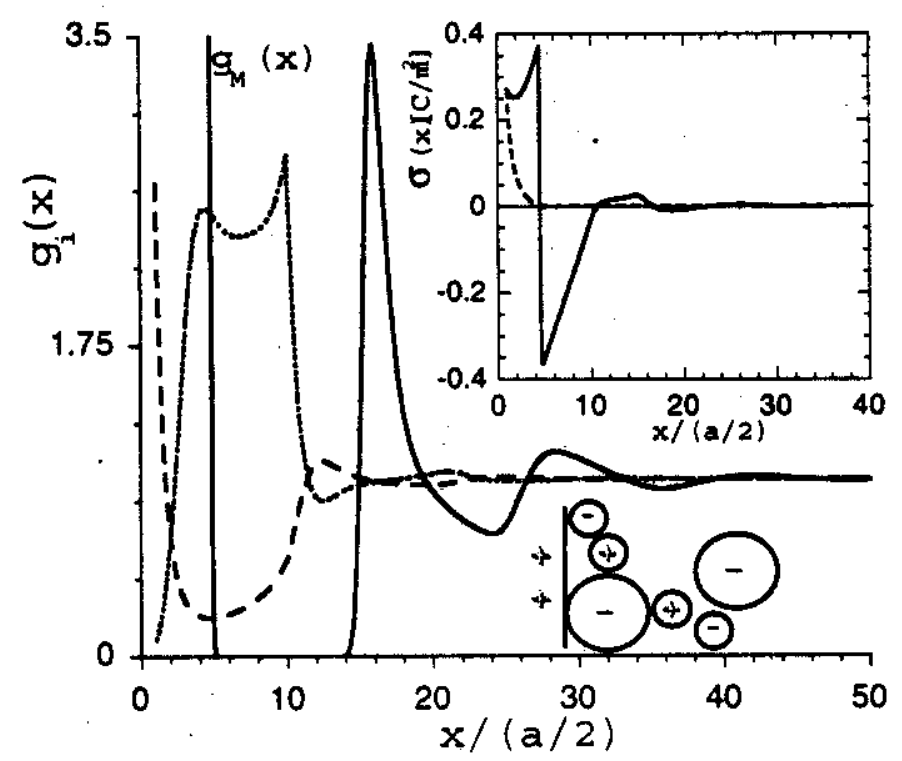

Figura 4.2: Perfil reducido de concentración para macroiones en una solución electrolítica $2: 1$, como una función de la distancia a la pared $\rho_{M}=0.01 M, \rho_{+}=0.7 M, \rho_{-}=1.0 M$, $\sigma_{0}=0.272 \mathrm{C} / \mathrm{m}^{2}, a_{M}=4.5 a, Z_{M}=-40, z_{+}=2, z_{-}=-1$. Las líneas sólida, intermitente y punteadas representan los PRC para los macroiones $(M)$, iones positivos $(+)$ y iones negativos $(-)$, respectivamente. En el recuadro la línea sólida es el perfil de densidad de carga efectiva, $\sigma(x)$, como una función de la distancia a la pared, para la solución de macroiones, mientras que, la línea intermitente es $\sigma(x)$ para un electrolito tipo MPR 2:1 con $\left(\rho_{+}=0.5 M, \rho_{-}=\right.$ $1.0 M, z_{+}=2, z_{-}=-1$ ), cuando no hay macroiones en la solución. El dibujo, representa someramente la distribución de iones indicados por sus PRC. 
ha sido reportado anteriormente y es probable que solo esté presente en soluciones de macroiones. El campo elèctrico efectivo, el cual es proporcional a $\sigma(x)$, es positivo antes de la primera capa de macroiones y posteriormente regativo, antes de la segunda capa. De esta forma, la fuerza eléctrica es primeramente atractiva y posteriormente repulsiva a los iones negativos. Sin embargo, el comportamientc de la fuerza total sobre un ion de la especie $i$, está implícito en el PRC, i.e., un valor de $g_{i}(x)$ por encima (debajo) de 1 implica que $F_{i}(x)$ es atractiva (repulsiva). En $x=a / 2, \sigma(x)$ es igual a la carga de la pared, mientras que para $x \rightarrow \infty, \sigma(x) \rightarrow 0$, como debe ser para satisfacer electroneutr llidad. Una densidad de carga menor sobre la pared así como una menor carga de los macroiones. tamaño o concentración produce una menor adsorción.

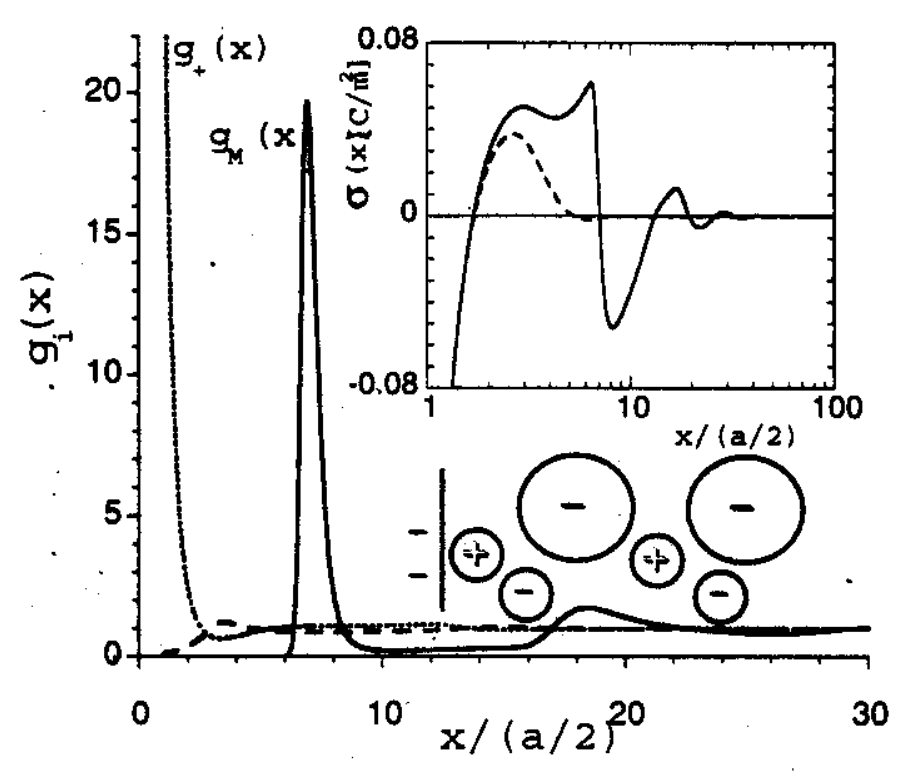

Figura 4.3: Como en la Fig. 4.2, pero, $\sigma_{0}=-0.272 \mathrm{C} / \mathrm{m}^{2}$. En el recuadro, la línea discontinua es $\sigma(x)$ para un electrolito 2:1 tipo MPR, cuando no hay macroiones en la solución, $\rho_{+}=$ $0.7 . \mathrm{H}, \rho_{-}=1.4 \mathrm{M}, z_{+}=2, z_{-}=-1$.

En la Fig. 4.3. la pared y los macroiones tienen carga negativa. Aquí los iones positivos divalentes son contraiones de la pared así como de los macroiones. Una capa de iones positivos. seguida por iones negativos es adsorbida sobre la pared. Posteriormente una fuerte adsorción de macroiones es observada. En el recuadro, un RC es seguido por una IC. En la Fig. 4.2, los macroiones son responsables de la primera RC, mientras que en la Fig. 4.3, son responsables de la primera CI. El electrolito 2:1 no muestra RC. De esta forma, en ausencia de macroiones, los contraiones rnonovalentes no producen RC (como se muestra en la Fig. 4.2), mientras que contraiones divalentes sí. El primer pico corresponde a una concentración de los macroiones de $0.2 \mathrm{M}$. Esto implica una atracción efectiva de largo aicance entre los macroiones adsorbidos. Esta atracción es menor conforme la 


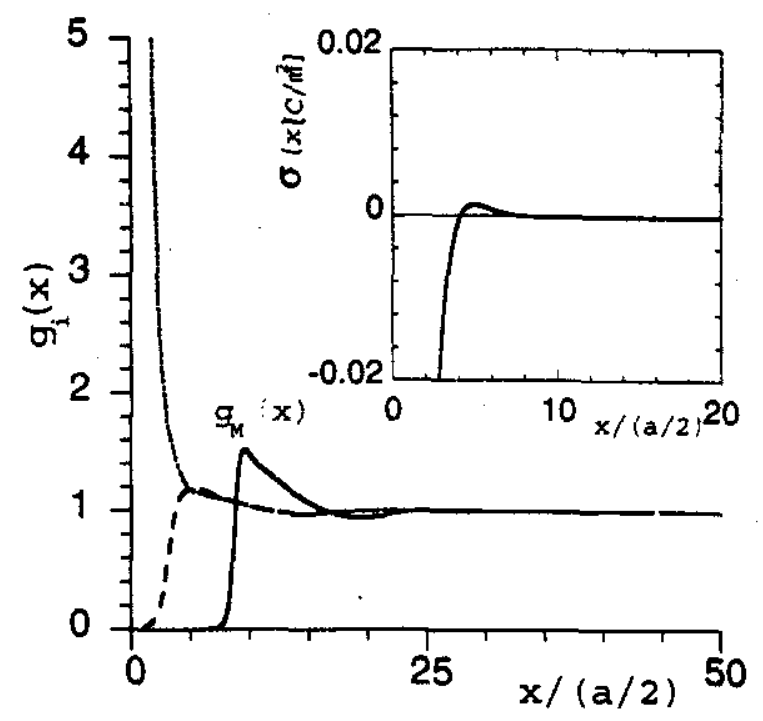

Figura 4.4: Como en la Fig. 4.2 pero la sal es un electrolito tipo MPR 1:1 y $\rho_{+}=1.0 M$, $\rho_{-}=1.0 M, \sigma_{0}=-0.272 \mathrm{C} / \mathrm{m}^{2}, a_{M}=6.5 a, Z_{M}=0, z_{+}=1, z_{-}=-1$.

distancia entre los macroiones y la pared se incremente o para macroiones más pequeños. Nuestros resultados muestran que la adsorción de macroiones depende de $\rho_{T}^{*} \equiv \rho_{+} a_{+}^{3}+$ $\rho_{-} a_{-}^{3}+\rho_{M} a_{M}^{3}$, i.e., del volumen excluido. Por lo tanto, la adsorción de macroiones más grandes implica una atracción de mayor largo alcance así como una atracción efectiva macroión-macroión mayor. Estos hallazgos son sugerentes en relación a la atracción entre macroiones igualmente cargados en presencia de una pared igualmente cargada, donde macroiones confinados cuyos tamaños son de alrededor de $1 \mu \mathrm{m}$ y muestran el mismo comportamiento [119].

En la Fig. 4.4, se muestran los resultados para macropartículas descargadas en una solución electrolítica 1:1, en presencia de una superficie cargada negativamente. En el recuadro se puede observar una IC muy tenue, aun cuando los contraiones son iones monovalentes. Los estudios de la DCE para un electrolito 1:1, demuestran que no aparecen oscilaciones de carga o en los PRC bajo estas condiciones, en el electrolito simple[69]. Por lo tanto, las oscilaciones en la Fig. 4.4 de los PRC de los iones pequeños se deben a las correlaciones por tamaño relacionadas con las macropartículas adsorbidas en la pared. Aquí podemos ver que la carga del macroión no es necesaria para tener adsorción de éstos y que su presencia induzca oscilaciones en los PRC del electrolito 1:1.

En la Fig. 4.5 la pared está descargada. Dado que el macroión tiene mavor tamaño que en los casos anteriores, $a_{M}=6.5 a$, hemos incrementado la carga de los macroiones para alcanzar la misma densidad superficia de carga equivalente. Sin embargo, el parárnetro de acoplamiento macroión-macroiór ${ }^{3}, \zeta$, se incrementa por un factor de 3 aproximadamente, respecto al correspondiente a $a_{3}:=4.5$ y $Z_{M}=-40$. Como resultado, tenemos una fuerte

\footnotetext{
${ }^{3} \mathrm{El}$ cual se discutió en el capítulo 3 , en donde $\eta$ y $\zeta$ aparecen como los parámetros relevantes en la descripción del sobrecargado. En este caso el parámetro de acoplamiento relevante es el de los macroiones,
} 


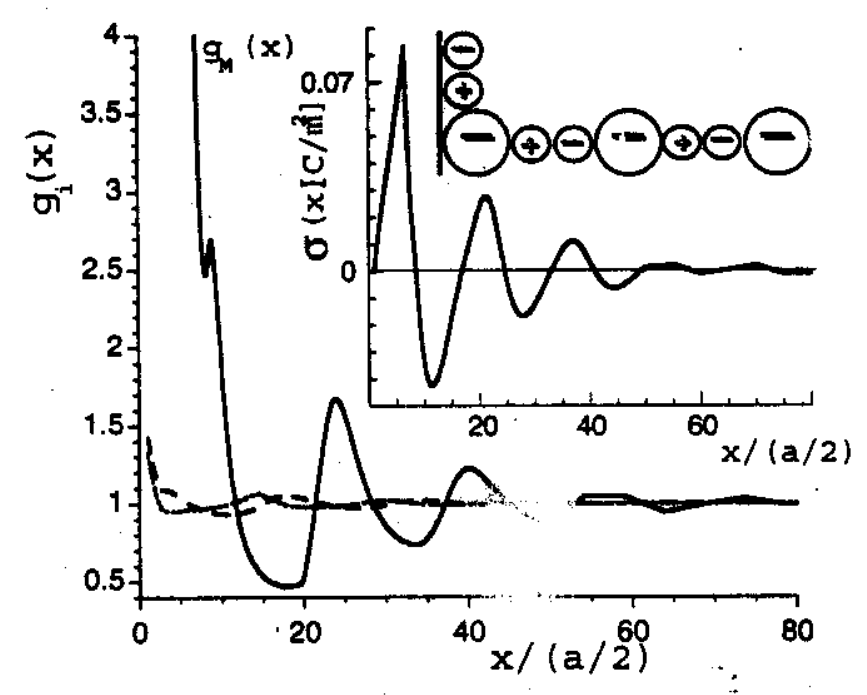

Figura 4.5: Como en la Fig. 4.2 pero $\rho_{+}=0.915 \mathrm{M}, \rho_{-}=1.0 \mathrm{M}, \sigma_{0}=0.0 \mathrm{C} / \mathrm{m}^{2}, a_{M}=6.5 a$, $Z_{M}=-83$.

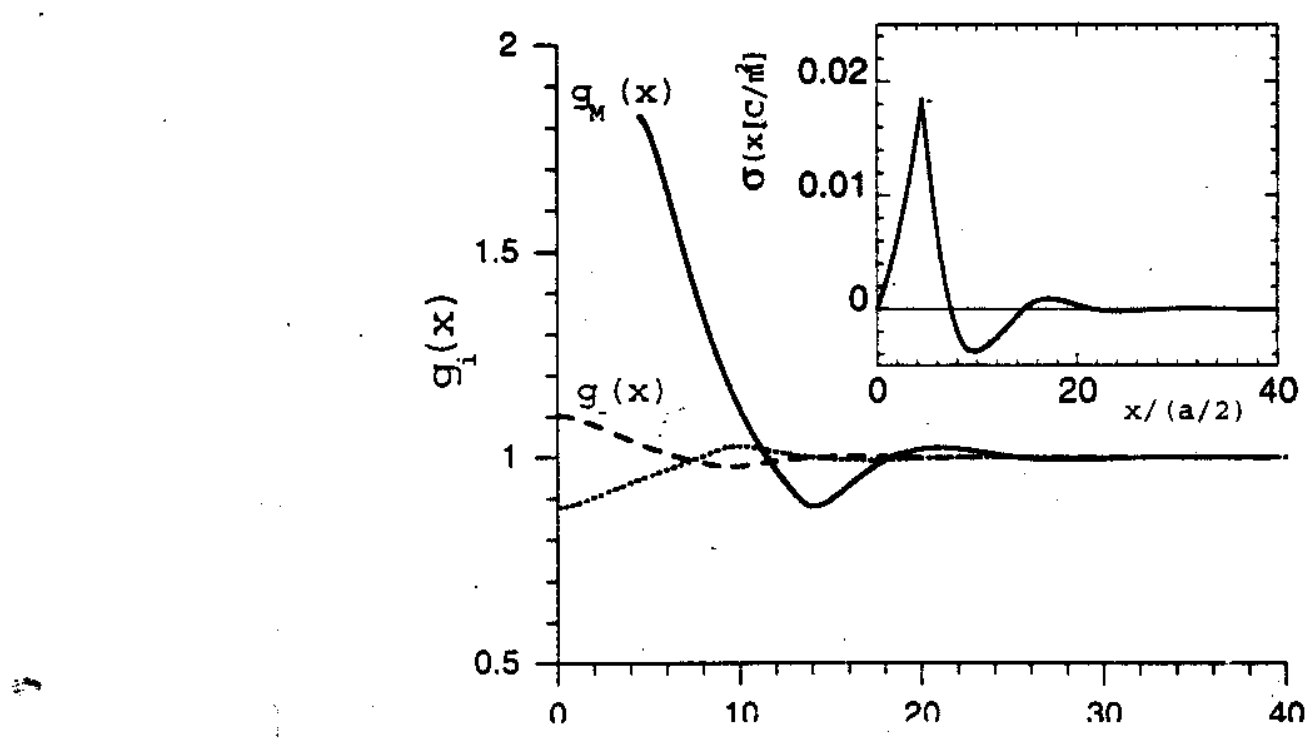

Figura 4.6: Como en la Fig. 4.2 pero la sal es un electrolito 2:I de iones puntuales $\sigma_{0}=$ $0.0 \mathrm{C} / \mathrm{m}^{2}, Z_{M}=-40$. 
adsorción de macroiones en la pared. La concentraciór: de los macroiones en el contacto con la pared es de $\approx 6.63 \mathrm{M}$. En el recuadro, st muesira un fuerte sobrecargado, tanto positivo como negativo de la pared. Si $a_{M}=4.5 x$ y $h_{M}=-40$ (no mostrado), el máximo de la segunda capa de macroiones decrece de 17, eil la Fig. 4.5, a 1.2, pero los PRC y la curva de $\sigma(x)$ son cualitativamente iguales. Si n . hay macroiones presentes, $\sigma(x)=$ $0, \forall x>0$. Dado que la superficie está descargada, 1 , fuerte adsorción de macroiones así como el sobrecargado se debe tanto al fuerte acople miento macroión-macróón como al muy alto volumen excluido, impuesto por el tamañ. de los macroiones. Da esta forrua se confirma que, a menor volumen accesible, el cual implica menor entropia, injose mas orden sobre la interfase, i. e., más adsorción sobre la superficie.

En la Fig: 4.6, presentamos los mismos cálculos que en la Fig. 4.5, pero ahora las especies del electrolito tienen dis: o igual a cero, mientras que los macroiones mantienen su mismo diámetro, $a_{M}=4.5 a$. Sice caso corresponde a una extensión de la teoría de Poisson-Boltzmann, donde únicamente dos macroiones son considerados [21]. En el recuadro, para $x<a_{3} / 2$, se presenta sobrecargado positivo. El máximo está en $x=a_{M} / 2$. El máximo de sobrecargado negativo está en $x \approx 9.6 a / 2$. Si los macroiones estuvieran ausentes de la solución, $\sigma(x)=0, \forall x>0$. Nótese las oscilaciones en los PRC de los iones puntuales. Este comportamiento oscilatorio está de acuerdo con la condición del segundo momento de Stillinger y Lovett [120], el cual es un resultado exacto. Sin embargo, es bien sabido que los PRC obtenidos de la teoría de Poisson-Boltzmann para iones puntuales tanto en el bulto como en presencia de una pared cargada o alrededor de dos macroiones (teoría DLVO $[34,118]$ ), no presentan oscilaciones y la interacción macroión-macroión es puramente repulsiva $[117,118]$. En efecto, es un resultado matemático exacto el hecho de que la ecuación de PB no puede predecir una fuerza atractiva entre dos macroiones igualmente cargados a dilusión infinita, entre ellos y con la pared [92]. La adsorción de macroiones hacia la pared implica una atracción efectiva entre ellos y con la pared. La diferencia de nuestro resultado de la Fig. 4.6 con el resultado de la teoría clásica de PB es la concentración finita de los macroiones, la cual implica una consideración propia de la entropía.

\subsection{Conclusiones de capítulo}

El parâmetro de acoplamiento entre los macroiones e cala como el cuadrado de la carga $y$ es inversamente proporcional al diámetro eatse os iones. De esta forma, aunque un ion divalente de diámetro $a=4.25 \AA$, tiene un । censidiad superficial de carga equivalente de $\sigma_{\mathrm{d}}=0.56 \mathrm{C} / \mathrm{m}^{2} \mathrm{y}$ un macroión de valencia $Z_{\lambda_{1}}=40 \mathrm{Y}$ diámetro $a_{M}=4.5 a$, tiene una densidad superficial de carga aproximadamene iglal $\left(c_{\mathrm{M}}=0.55 \mathrm{C} / \mathrm{m}^{2}\right)$; los parámetros de acoplamiento son $\zeta=6.72$ y $\zeta_{M} \approx 600$, para los iones divalentes y macroiones, respectivamente. De esta forma, dado que la arlsorciór así como los efectos de IC, RC y

$\zeta_{M}=\frac{3 q_{M}^{2}}{\varepsilon a_{M}}$. 
sobrecargados dependen de estos parámetros además del volumen excluido, observamos que estos efectos se magnifican en un fluido con macroiones.

En nuestros resultados podemos observar que la adsorción de macroiones puede incrementarse por dos mecanismos: (i) incrementando el volumen excluido del sistema, el cual puede llevarse a cabo incrementando el tamaño o la concentración ${ }^{4}$ o (ii) incrementando el parámetro de acoplamiento entre los macroiones $\zeta_{M}$. De esta forma, dado que para un fluido con macroiones estos parámetros pueden variar en un rango de valores muy amplio, en nuestros resultados encontramos los siguientes efectos 1) autoensamblado de macroiones en una superficie cargada, 2) atracción efectiva de largo alcance entre una superficie y macroiones igualmente cargados 3) al incrementar el volumen excluido encontramos IC en sistemas que normalmente no lo presentan 4) formación de estructuras autoensambladas (layering) en una superficie desces a y finalmente 5) oscilaciones en los perfiles de concentración de los iones puntuales d das a las correlaciones por tamaño y carga con los macroiones.

Nuestros resultados muestran que el efecto de volumen excluido es mucho más importante de lo que ha sido reconocido anteriormente. De esta forma, RC, IC, y el sobrecargado resultan de una competencia entre entropía y energía. Finalmente nuestros resultados proveen de algunos mecanismos que pueden ocurrir o conducir a las observaciones en los experimentos de Grier donde se encuentra atracción de largo alcance entre macroiones confinados igualmente cargados [119] así como sobre el. autoensamblado de polímeros en una superficie $[112,121$ y la formación de estructuras complejas de ADN [34].

\footnotetext{
${ }^{4} \mathrm{El}$ volumen excluido depende de $\rho_{T}^{*} \equiv \rho_{+} a_{+}^{3}+\rho_{-} a_{-}^{3}+\rho_{M} a_{M}^{3}$
} 


\section{Capítulo 5}

\section{La función de correlación de tres partículas para a electrolito}

\subsection{Introducción}

En la literatura hay dos propuestas principales usadas para el estudio de fluidos homogéneos: las ecuaciones de la jerarquia y el formalismo de Ornstein-Zernike[57, 58]. Ambas propuestas proveen de teorías para el cálculo de $g^{(2)}\left(\mathbf{r}_{1}, \mathbf{r}_{2}\right)$. En las teorías derivadas de la jerarquía tales como la ecuación de Born-Green-Yvon (BGY) $[122,123], g^{(n)}$ es expresada en términos de $g^{(n+1)}$. Por lo que una relación entre las dos funciones es necesaria. Una de las aproximaciones más usadas en esta clase de teorías, es la aproximación de superposición de Kirkwood(ASK). En ASK [124], se supone aditividad por pares a nivel del potencial de la fuerza promedio. La comparación de las propiedades termodinámicas calculadas a través de la teoría BGY y por medio de simulación molecular, para un fluido denso de esferas duras, muestran la falta de acuerdo $[53,125]$.

Como ya mencionamos en el capitulo 1, en el formalismo de Ornstein-Zernike debe emplearse una función de cerradura entre la función de correlación total y la función de correlación directa. Dependiendo de la función de cerradura usada se pueden derivar diferentes teorias de ecuaciones integrales. Entre las más conocidas están la teoria de Percus-Yevick (PY) [126], la de cadena hipertejida (HNC) y la aproximación esférica media (MSA) [127]. a un desarrollo en diagramas de Maver, se puede ver que todas estas teorias ignoran diferentes clases de diagramas $[52,5 j]$. I a comparación con simulación molecular siempre es requerida para evaluar el impacto de despreciar tales términos. En una comparación con simulación molecular de las propiedades termodinámicas de un fluido de esferas duras obtenidas por medio de HNC y $\mathrm{P}^{\prime} /$, m restran una mejoría respecto a los resultados obtenidos de las teorías de la jerarcuía pero aún fallan en su descripción a altas concentraciones $[53,125]$.

Estudios de las propiedades termodinámicas del modelo primitivo restringido, se han llevado a cabo anteriormente a través de las teorís HNC [128, i29, 130], MSA [131, 132] y BGY [133]. Todas estas teorías describen bien' el MPR bajo ciertas condiciones pero 
fallan, principalmente, cuando las correlaciones de largo alcance se vuelven importantes [47]. Esto evidencía la necesidad de tener una teoría que describa correctamente a los sistemas cuando las correlaciones de largo alcance son relevantes.

El propósito del presente capítulo es presentar al formalismo de extensión a tres puntos (TPE) como un método para sumar correlaciones en una teoria dada de ecuaciones integrales. El formalismo de extensión a tres puntos ha sido aplicado exitosamente para calcular la fuerza entre dos placas paralelas cargadas, inmersas en un electrolito $[16,15,134]$. También la extensión a tres puntos de la versión integral de la ecuación de Poisson-Boltzmann, se ha utilizado para calcular la fuerza como función de su separación, entre dos partículas coloidales esféricas igualmente cargadas e inmersas en un electrolito $[17,18,19,118]$. Se ha demostrado para el caso de las placas paralelas [16], que este método representa una mejora en la fuerza calculada con respecto a la calculada con ASK.

En este capítulo desarrollamos los detalles matemáticos necesarios para aplicar el formalismo de extensión a tres puntos a un electrolito a través de la teoría HNC/MSA. Como resultado obtenemos la función de correlación condicional de tres partículas, $g_{\beta \gamma i}^{[3]}\left(\mathbf{r}_{1}, \mathbf{r}_{2}, \mathbf{r}_{3}\right)$, de la cual presentamos resultados numéricos. Por otra parte, hemos calculado $g_{\beta \gamma i}^{[3]}\left(\mathbf{r}_{1}, \mathbf{r}_{2}, \mathbf{r}_{3}\right)$ a través de dinámica molecular, con los que comparamos los resultados de TPE. Por último, para presentar estudios a nivel de la función de correlación por pares, hemos calculado . la fuerza promedio entre las dos partículas como una función de su separación. Los resultados de TPE los comparamos con DME y con los obtenidos por la teoría estándar $\mathrm{HNC} / \mathrm{MSA}$.

\subsection{Extensión a tres puntos de las ecuaciones integrales}

La extensión a tres puntos de las ecuaciones integrales es un método para incorporar un número casi infinito de correlaciones en una teoría dada de ecuaciones integrales. La idea básica es que podemos utilizar la ecuación de Ornstein-Zernike para obtener funciones de distribución de orden superior, es decir, en la primera extensión se puede calcular una función de correlación de tres partículas. Partiendo del hecho de que las teorías que hemos mencionado en el capítulo 1 no están restringidas respecto al número de especies en el fluido, el potencial de interacción entre las partículas o la geometría de las especies; se puede pensar en un fluido formado por $(n+1)$ especies, en el cual, una de las especies se construye artificialmente como una mancuerna formada por dos partículas (de especie $\alpha$ y 3 ) fijas a una distancia dada entre sus centros, $\tau \equiv\left|\mathbf{r}_{1}-\mathbf{r}_{2}\right|$ (ver Fig. j.1). Si llamamos a la especie artificial $\alpha$ y tomamos el límite $\rho_{\alpha} \rightarrow 0$. la ecuación de Ornstein-Zernike para la función de correlación entre la especie $\alpha$ y $j$ está dada por

$$
h_{\alpha j}\left(\mathbf{r}_{3}\right)=c_{\alpha j}\left(\mathbf{r}_{3}\right)+\sum_{m=1}^{n} \rho_{m} \int h_{\alpha m}\left(\mathbf{r}_{4}\right) c_{m j}\left(\mathbf{r}_{34}\right) d \mathbf{r}_{4} .
$$

Las especies restantes satisfacen una ecuación de Ornstein-Zernike como la Ec. (1.21). En la integral de la Ec. (5.1) las funciones $c_{\alpha j}\left(\mathbf{r}_{34}\right)$ y $c_{\alpha \alpha}\left(\mathbf{r}_{34}\right)$ no aparecen, sino solamente 
las funciones $c_{m j}\left(\mathbf{r}_{34}\right)$. Las primeras son las funciones de correlación mancuerna-fluido y mancuerna-mancuerna, mientras que las últimas son las funciones de correlación directa entre las especies del fluido. Para obtener $c_{m j}\left(\mathbf{r}_{34}\right)$, una ecuación tipo Ornstein-Zernike para $n$ especies tiene que resolverse de manera independiente. Diferentes teorías de ecuaciones integrales pueden obtenerse [56] dependiendo de la relaciones de cerradura usadas para $c_{\alpha j}\left(\mathbf{r}_{3}\right)$ y $c_{m j}\left(\mathbf{r}_{34}\right)$ en la Ec. (5.1). Por cojemplo, la ecuación TPE-HNC/MSA se obtiene si MSA, i. e., Ec. (1.23), se usa para $c_{m j}\left(\mathbf{r}_{34}\right)$ y HNC, i. e., Ec. (1.22), para $c_{\alpha i}\left(\mathbf{r}_{3}\right)$.

La función de distribución $g_{\alpha i}\left(\mathbf{r}_{3}\right)=h_{\alpha i}\left(\mathbf{r}_{3}\right)+1$ de la especie $i$ alrededor de la partícula $\alpha$ obtenida de la solución de la Ec. (5.1) se interpreta como una función de distribución condicional de tres partículas y se denota como $g_{\beta \gamma i}^{[3]}\left(\mathbf{r}_{1}, \mathbf{r}_{2}, \mathbf{r}_{3} ; \tau=\left|\mathbf{r}_{1}-\mathbf{r}_{2}\right|\right)$, i. e., la densidad de probabilidad de una partícula de la especie $i$ en $\mathbf{r}_{3}$, cuando las partículas $\beta$ y $\gamma$ están en $\mathbf{r}_{1}$ y $\mathbf{r}_{2}$ (fijas con una posición relativa $\tau$ ), respectivamente. Este artificio es conocido como extensión a tres puntos (por la forma que adquiere el desarrollo de $g_{\alpha i}$ en diagramas de Mayer, lo cual discutiremos en la sección 5.3). La Ec. (5.1) es la extensión a tres puntos de la ecuación de Ornstein-Zernike. Matemáticamente la función de distribución condicional $g_{\beta \gamma i}^{[3]}$ está relacionada con la función de correlación homogénea de tres partículas, $g_{\beta \gamma i}^{(3)}$, por

$$
g_{\beta \gamma i}^{[3]}\left(\mathbf{r}_{1}, \mathbf{r}_{2}, \mathbf{r}_{3} ; \tau=\left|\mathbf{r}_{1}-\mathbf{r}_{2}\right|\right)=\frac{g_{\beta \gamma i}^{(3)}\left(\mathbf{r}_{1}, \mathbf{r}_{2}, \mathbf{r}_{3}\right)}{g_{\beta \gamma}^{(2)}\left(\mathbf{r}_{1}, \mathbf{r}_{2}\right)}
$$

La proyección de $g_{\beta \gamma i}^{[3]}$ da como resultado $g_{\beta \gamma}^{(2)}$. Esta proyección la provee la ecuación de Born-Green-Yvon (BGY), la cual está basada en un balance de la fuerza efectiva promedio entre dos partículas (de especies $\beta$ y $\gamma$ ) $\mathrm{F}_{\beta \gamma}\left[g_{\beta \gamma i}^{(3)}\right][122,123]$. De donde a su vez, se puede obtener la información de $g_{\beta \gamma}^{(2)}$, de acuerdo con la Ec. (1.12).

\subsection{La ecuación BGY como una ley de fuerzas}

La ecuación de Born-Green-Yvon (BGY) es una de las llamadas ecuaciones de la jerarquía, la cual es un teorema exacto que relaciona las funciones de correlación de $n \mathbf{y}(n+1)$ partículas. En esta sección deducimos la ecuación BGY como un resultado de la fuerza efectiva sobre una partícula de la mancuerna (digamos la partícula de la especie $\gamma$ en $\mathbf{r}_{2}$ ) $F_{\beta \gamma}$.

Esta interacción tiene dos contribuciones: la interacción directa $\mathbf{f}_{\beta \gamma}$ y la interacción a través de las partículas que rodean a la mancuerna $\mathbf{f}_{\gamma}^{\mathrm{d}}$. De tal forma que la fuerza total, $\mathbf{F}_{\beta \gamma}$, se escribe como

$$
\mathbf{F}_{\beta \gamma}=\mathbf{f}_{\beta \gamma}+\mathbf{f}_{\gamma}^{\mathrm{d}},
$$

Suponiendo que las partículas que forman la mancuerna son de geometria esférica y que 


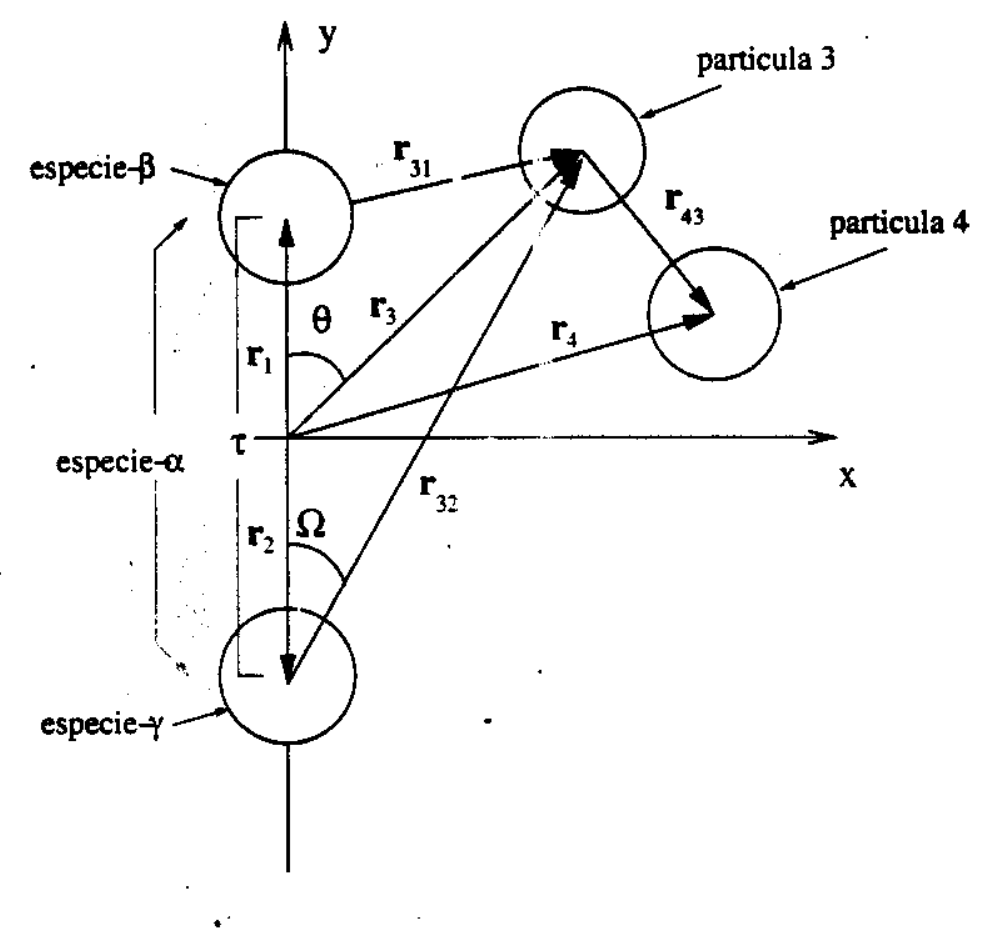

Figura 5.1: Representación esquemática de nuestro modelo.

interaccionan a través de potenciales de fuerzas centrales, la componente de $\mathbf{f}_{\beta \gamma}$ a lo largo del vector eje de la mancuerna $\tau$, está dada por

$$
\mathrm{f}_{\beta \gamma}=-\frac{\mathrm{d} u_{\beta \gamma}(\tau)}{\mathrm{d} \tau}
$$

donde $u_{\beta \gamma}(\tau)$ es el potencial de interacción directa entre las partículas que forman la mancuerna. Si hay $\rho_{i}\left(\mathbf{r}_{3}\right)$ partículas por unidad de volumen de la especie $i$ en $\mathbf{r}_{3}$, el número de partículas de dicha especie en un elemento de volumen $\mathrm{d} v_{3}$ es $\mathrm{d} n_{i}\left(\mathbf{r}_{3}\right)=\rho_{i}\left(\mathbf{r}_{3}\right) \mathrm{d} v_{3}$. Si $\mathbf{f}_{i \gamma}\left(\mathbf{r}_{23}\right)$ es la fuerza entre la especie $i$ y la partícula de prueba de la mancuerna en $\mathbf{r}_{2}$ (de la especie $\gamma$ ), la fuerza ejercida por un elemento de fluido en $\mathbf{r}_{3}$ sobre la partícula de

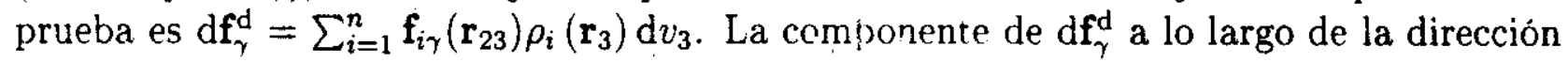
de $\tau$ está dada por

$$
d \mathrm{f}_{\gamma}^{\mathrm{d}}=\sum_{i=1}^{n} \hat{\tau} \cdot \mathbf{f}_{i \gamma}\left(\mathbf{r}_{23}\right) \rho_{i}\left(\mathbf{r}_{3}\right) d v_{3}=-\sum_{=1}^{n} \hat{\tau} \cdot \hat{\mathbf{r}}_{23} \frac{\mathrm{d} u_{\gamma i}\left(\mathbf{r}_{23}\right)}{d r_{23}} \rho_{i}\left(\mathbf{r}_{3}\right) \mathrm{d} v_{3},
$$

siendo $\hat{\tau}$ y $\widehat{\mathbf{r}}_{23}$ los vectores unitarios a lo :argo de los cirecciones $\boldsymbol{\tau}$ y $\mathbf{r}_{23}$, respectivamente, $u_{i \gamma}\left(\mathbf{r}_{23}\right)$ es el potencial de interacción directa eitre una partícula de la especie $i$ con la partícula de especie $\gamma ; \rho_{i}\left(\mathbf{r}_{3}\right)$ es la conceat ación luç.l. i. e.,

$$
\rho_{i}\left(\mathbf{r}_{3}\right):=\rho_{i} g_{3 \gamma i}^{3 !}\left(\mathbf{r}_{3,}, i\right)
$$

Sustituyendo las Ecs.(5.4) y (5.5) en la lc.(5.3). la fuerza media sobre la partícula $\gamma$ es 


$$
\mathbf{F}_{\beta \gamma}=-\frac{\mathrm{d} w_{\beta \gamma}(\tau)}{\mathrm{d} \tau}=-\frac{\mathrm{d} u_{\beta \gamma}(\tau)}{\mathrm{d} \tau}-\sum_{i=1}^{n} \rho_{i} \int \frac{\mathrm{d} u_{\gamma i}\left(\mathbf{r}_{23}\right)}{\mathrm{d} r_{3}} \cos \Omega g_{\beta \gamma i}^{[3]}\left(\mathbf{r}_{3}, \tau\right) \mathrm{d} v_{3},
$$

donde $w_{\beta \gamma}(\tau)$ es el potencial de la fuerza promedio entre las partículas de la mancuerna, y se ha usado el hecho de quc $\cos \Omega=\widehat{\tau} \cdot \widehat{\mathbf{r}}_{23}$. De acuerdo con la Ec. (1.20), tenemos

$$
w_{\beta \gamma}(\tau)=-k_{B} T \ln \left[g_{\beta \gamma}(\tau)\right] .
$$

De tal forma que la relación entre $g_{\beta \gamma i}^{[3]}$ y $g_{\beta \gamma}^{(2)}$ se escribe como

$$
\hat{n}_{B} T \frac{\mathrm{d} \ln g_{\beta \gamma}^{(2)}(\tau)}{\mathrm{d} \tau}=-\frac{\mathrm{d} u_{\beta \gamma}(\tau)}{\mathrm{d} \tau}-\sum_{i=1}^{n} \rho_{i} \int \frac{\mathrm{d} u_{\gamma i}\left(\mathbf{r}_{23}\right)}{\mathrm{d} r_{23}} \cos \Omega g_{\beta \gamma i}^{[3]}\left(\mathbf{r}_{3}, \tau\right) \mathrm{d} v_{3},
$$

la cual es la ecuación Born-Green-Yvon (BGY) derivada como una suma de fuerzas. Si $g_{\beta \gamma i}^{[3]}$ es calculada a través de el formalismo de extensión a tres puntos de las ecuaciones integrales, se puede demostrar que nuevos diagramas son incluidos en un desarrollo de Mayer de $g_{\beta \gamma}^{(2)}[56]$.

En la Fig. 5.2 se muestra transformación de un diagrama de Mayer bajo la aplicación del formalismo de la extensión a tres puntos. La línea punteada que se presenta en el diagrama resultante corresponde a la función $\frac{\mathrm{d} u_{\gamma i}\left(\mathbf{r}_{23}\right)}{\mathrm{d} r_{3}}$, que aparece en el término integral de la Ec. (5.9). Tras examinar la evolución de una diagrama de Mayer bajo el formalismo propuesto, es clara la denominación de extensión a tres puntos.

\subsection{Aplicación al modelo primitivo restringido}

En el electrolito MPR, la condición de electroneutralidad para un electrolito de $n$ componentes es

$$
\sum_{i=1}^{n} z_{i} \rho_{i}=0
$$

Suponiendo que la mancuerna (especie $\alpha$ ) está formada de dos partículas de la misma especie que las del fluido, comc en la Fig. 5.1, la ecuación TPE-HNC/MSA está dada por

$$
g_{\alpha i}\left(\mathbf{r}_{3}\right)=\exp \left\{-\beta u_{\alpha}\left(\mathbf{r}_{3}\right)+\sum_{m=1}^{k} \rho_{m} \int h_{\alpha m}\left(\mathbf{r}_{4}\right) c_{m i}\left(\mathbf{r}_{34}\right) d \mathbf{r}_{4}\right\}
$$

donde $u_{\alpha i}\left(\mathbf{r}_{3}\right)$ es el potencial de interacción directa entre la mancuerna y las partículas del fluido de la especie $i$, dado por

$$
u_{\alpha i}\left(\mathbf{r}_{3}\right)=u_{\alpha i}\left(r_{13}, r_{: 33}\right)
$$



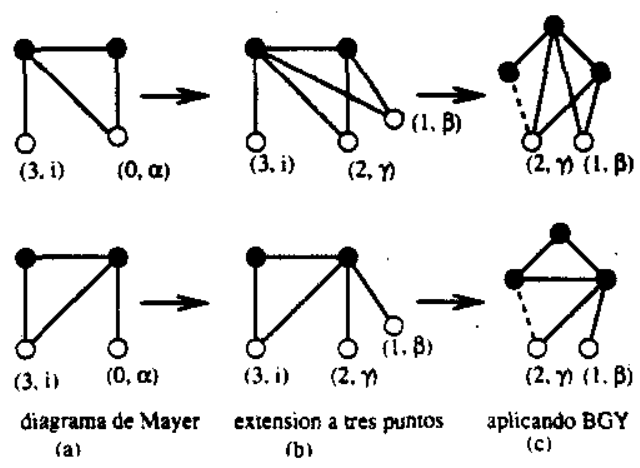

Figura 5.2: Dos ejemplos de la transformación de un diagrama de Mayei bajo la aplicación del formalismo de extensión a tres puntos. La notación para las partículas y las especies es la misma que en la Fig. 5.1. La liga punteada representa la función $f_{\gamma i}=\frac{\mathrm{d} u_{\gamma i}\left(\mathbf{r}_{23}\right)}{\mathrm{d} r_{23}}$. De esta forma, en $(N, \delta), N$ representa el número de partícula y $\delta$ la especie (ver Fig. 5.1). (a) Un ejemplo de un diagrama de Mayer que involucra a la mancuerna y la partícula 3. (b) El mismo diagrama que en (a) pero las partículas que forman la mancuerna son explícitamente mostradas, i. e., a nivel de la función de distribución de tres partículas. (c) Diagramas resultantes bajo la aplicación de BGY (a nivel de la fuerza promedio)

$$
=\left\{\begin{array}{l}
\frac{z_{i} z_{\beta} e^{2}}{\varepsilon r_{23}}+\frac{z_{i} z_{\gamma} e^{2}}{\varepsilon r_{13}} \text { si } r_{13} \text { and } r_{23}>a \\
\infty \quad \text { si } r_{13} \text { or } r_{23} \leq \dot{a}
\end{array}\right.
$$

siendo $z_{\beta}$ y $z_{\gamma}$ la valencia de las especies $\beta$ y $\gamma$, respectivamente. Para iones esféricos la función de correlación directa depende únicamente de su distancia relativa, $r_{34}=\left|\mathbf{r}_{34}\right|$. En la aproximación esférica media ésta tiene una expresión analítica, la cual se puede escribir como

$$
c_{m i}\left(r_{34}\right)=c^{h s}\left(r_{34}\right)+z_{m} z_{i} c^{s r}\left(r_{34}\right)-\beta \frac{z_{m} z_{i} e^{2}}{\varepsilon r_{13}},
$$

donde $c^{h s}\left(r_{34}\right)$ es la función de correlación directa para un fluido de esferas duras en la aproximación de PY, y $c^{8 r}\left(r_{34}\right)$ es una función de corto alcance, las cuales están dadas en el apéndice B. Se ha propuesto utilizar coordenadas prolatas [135] para esta geometría, las cuales están definidas de la siguiente forma

$$
\begin{aligned}
& x=\frac{\tau}{2} \sqrt{\left(\eta^{2}-1\right)\left(1-\xi^{2}\right)} \cos \phi .
\end{aligned}
$$

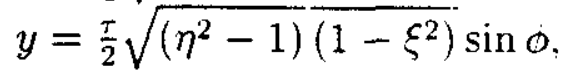

$$
\begin{aligned}
& z=\frac{\tau}{2} \eta \xi \text {, }
\end{aligned}
$$

donde el elemento de volumen está dado por 


$$
d v=\frac{\tau^{3}}{8}\left(\eta^{2}-\xi^{2}\right) d \phi d \xi d \eta
$$

y

$$
\begin{aligned}
r_{34}^{2}= & \frac{\tau^{2}}{4}\left\{\left(\eta_{3}^{2}-1\right)\left(1-\xi_{3}^{2}\right)+\left(\eta_{4}^{2}-1\right)\left(1-\xi_{4}^{2}\right)\right. \\
& +\left(\eta_{3} \xi_{3}-\eta_{4} \xi_{4}\right)^{2} \\
& \left.-2 \sqrt{\left(\eta_{3}^{2}-1\right)\left(1-\xi_{3}^{2}\right)\left(\eta_{4}^{2}-1\right)\left(1-\xi_{4}^{2}\right)} \cos \phi_{4}\right\}
\end{aligned}
$$

En este sistema de coordenadas, el potencial electrostático de interaction puede ser escrito de manera conveniente de la siguiente forma

$$
u_{\alpha i}(\eta, \xi)=\frac{2 e^{2}}{\tau \varepsilon}\left(\frac{z_{\beta}}{\eta-\xi}+\frac{z_{\gamma}}{\eta+\xi}\right)
$$

Tomando en cuenta la simetría alrededor del eje $z$, la Ec. (5.11) puede ser reescrita como.

$$
\begin{aligned}
g_{\alpha i}\left(\eta_{3}, \xi_{3}\right)= & g_{\beta \gamma i}^{[3]}\left(\eta_{3}, \xi_{3} ; \tau\right) \\
= & \exp \left\{-\frac{2 \beta e^{2}}{\tau \varepsilon}\left(\frac{z_{\beta}}{\eta_{3}-\xi_{3}}+\frac{z_{\gamma}}{\eta_{3}+\xi_{3}}\right)\right. \\
& +\int_{-1}^{1} \int_{\eta_{0}\left(\xi_{4}\right)}^{\infty} \rho_{\alpha s}\left(\eta_{4}, \xi_{4}\right) \mathrm{K}\left(\eta_{3}, \xi_{3}, \eta_{4}, \xi_{3}\right) \mathrm{d} \eta_{4} \mathrm{~d} \xi_{4} \\
& +z_{i} \int_{-1}^{1} \int_{\eta_{0}\left(\xi_{4}\right)}^{\infty} \rho_{\alpha \mathrm{d}}\left(\eta_{4}, \xi_{4}\right) \mathrm{L}\left(\eta_{3}, \xi_{3}, \eta_{4}, \xi_{4}\right) \mathrm{d} \eta_{4} \mathrm{~d} \xi_{4} \\
& \left.-z_{i} \int_{-1}^{1} \int_{\eta_{0}\left(\xi_{4}\right)}^{\infty} \rho_{\alpha \mathrm{d}}\left(\eta_{4}, \xi_{4}\right) \mathrm{A}\left(\eta_{3}, \xi_{3}, \eta_{4}, \xi_{i}\right) \mathrm{d} \eta_{4} \mathrm{~d} \xi_{4}-\mathrm{J}\left(\eta_{3}, \xi_{3}\right)\right\}
\end{aligned}
$$

donde

$$
\eta_{0}(\xi)=\left\{\begin{array}{lll}
\xi+b & \text { para } & \varepsilon_{0}<\xi \leq 1 \\
1 & \text { para } & 0 \leq \xi \leq \xi_{0}
\end{array}\right.
$$

con $\xi_{0}=1-b, b \equiv 2 a / \tau y \eta_{0}(-\xi)=\eta_{0}(\xi)$. Las expresiones para $\mathrm{K}, \mathrm{L}, \mathrm{A}, \mathrm{J}, \rho_{\alpha \mathrm{s}}$ y $\rho_{\alpha \mathrm{d}}$ son

$$
\begin{aligned}
\mathrm{K}\left(\eta_{3}, \xi_{3}, \eta_{4}, \xi_{4}\right) & =\frac{\tau^{3}}{8}\left(\dot{\eta}_{4}^{2}-\xi_{4}^{2}\right) \int_{0}^{\phi_{\max }} c^{\mathrm{hs}}\left(r_{34}\right) d \phi_{4}, \\
\mathrm{~L}\left(\eta_{3}, \xi_{3}, \eta_{4}, \xi_{4}\right) & =\frac{\tau^{3}}{8}\left(\eta_{4}^{2}-\xi_{3}^{2}\right) \int_{0}^{\phi_{\max }} c^{\mathrm{sr}}\left(r_{34}\right) d \phi_{4}, \\
\mathrm{~A}\left(\eta_{3}, \xi_{3}, \eta_{4}, \xi_{4}\right) & =-\frac{\tau^{4} 3 e^{2}}{8 \varepsilon}\left(\eta_{4}^{2}-\xi_{4}^{2}\right) \int_{0}^{2 \pi} \frac{d \phi_{4}}{r_{34}},
\end{aligned}
$$


$\mathrm{y}$

$$
\begin{gathered}
\rho_{\alpha \mathrm{s}}\left(\eta_{4}, \xi_{4}\right)=\rho_{\beta \gamma \mathrm{s}}\left(\eta_{4}, \xi_{4}\right) \equiv \sum_{m=1}^{n} \rho_{m} h_{\alpha m}\left(\eta_{4}, \xi_{4}\right) \\
\rho_{\alpha \mathrm{d}}\left(\eta_{4}, \xi_{4}\right)=\rho_{\beta \gamma \mathrm{d}}\left(\eta_{4}, \xi_{4}\right) \equiv \sum_{m=1}^{n} z_{m} \rho_{m} h_{\alpha m}\left(\eta_{4}, \xi_{4}\right)
\end{gathered}
$$

$\mathrm{y}$

$$
\begin{aligned}
J\left(\eta_{3}, \xi_{3}\right)= & \int_{-1}^{-\xi_{\min }(\tau)} \int_{1}^{\eta_{0}\left(\xi_{4}\right)} \mathrm{K}\left(\eta_{4}, \xi_{4}, \eta_{3}, \xi_{3}\right) d \eta_{4} d \xi_{4} \\
& +\int_{\xi_{\min }(\tau)}^{1} \int_{1}^{\eta_{0}\left(\xi_{4}\right)} \mathrm{K}\left(\eta_{4}, \xi_{4}, \eta_{3}, \xi_{3}\right) d \eta_{4} d \xi_{4}
\end{aligned}
$$

donde

$$
\begin{gathered}
\xi_{\min }(\tau)=\left\{\begin{array}{lll}
0 & \text { si } & \tau \leq a \\
\xi_{0} & \text { si } & \tau>a
\end{array}\right. \\
\mathrm{A}\left(\eta_{3}, \xi_{3}, \eta_{4}, \xi_{4}\right)=\frac{\tau\left(\eta_{3}^{2}-\xi_{3}^{2}\right) \mathrm{F}(\pi / 2, k)}{2 r_{34}^{\max }}
\end{gathered}
$$

donde $\mathrm{F}(\pi / 2 ; k)$ es la función elíptica de segundo tipo.

$$
\begin{gathered}
k^{2}=\frac{\tau^{2} \sqrt{\left(\eta_{3}^{2}-1\right)\left(1-\xi_{3}^{2}\right)\left(\eta_{4}^{2}-1\right)\left(1-\xi_{4}^{2}\right)}}{2\left(r_{34}^{\max }\right)^{2}} \\
\left(r_{34}^{\text {max }}\right)^{2}=\frac{\tau^{2}}{4}\left[\sqrt{\left(\eta_{3}^{2}-1\right)\left(1-\xi_{3}^{2}\right)}+\sqrt{\left(\eta_{4}^{2}-1\right)\left(1-\xi_{4}^{2}\right)}\right]^{2}+\left(\eta_{3} \xi_{3}-\eta_{4} \xi_{4}\right)^{2} .
\end{gathered}
$$

Usando la Ec. (5.7), la fuerza media entre las dos partículas $\beta$ y $\gamma$ se escribe como

$$
\mathrm{F}_{\beta \gamma}(\tau)=\mathrm{f}_{\beta \gamma}^{*}(\tau)+\mathrm{f}_{\beta \gamma}^{\mathrm{el}}(\tau)
$$

con

$+$

$$
\mathrm{f}_{3 \gamma}^{*}(\tau)=\frac{\pi \tau^{2}}{2 \beta} \sum_{j=1}^{2} \rho_{j} \int_{\xi_{\min }(\tau)}^{1} g_{\beta \gamma j}^{[3]}\left(\eta_{0}(\xi), \xi\right)\left[-2 \xi^{3}-3 b \xi^{2}+\left(2-b^{2}\right) \xi+b\right] d \xi
$$

$$
\mathrm{f}_{3 \gamma}^{\mathrm{el}}(\tau)=\frac{z_{\beta} z_{\gamma} e^{2}}{\varepsilon \tau^{2}}+\frac{\tau \pi z_{\beta} e^{2}}{\varepsilon} \int_{-1}^{1} \int_{\eta_{0}(\xi)}^{\infty} \rho_{\beta \gamma \mathrm{d}}(\eta, \xi) \frac{(1-\eta \xi)(\eta+\xi)}{(\eta-\xi)^{2}} d \xi d \eta
$$

De la Ec. (5.8) la función de correlación por pares se puede calcular a partir de

$$
g_{\beta \gamma}(r)=\exp \left\{-\beta \int_{\infty}^{\tau} \mathrm{F}_{\beta \gamma}(\tau) d \tau\right\}
$$




\subsection{Algunos detalles de la dinámica molecular}

El electrolito está confinado en una caja cúbica de longitud $\mathrm{L}_{\mathrm{b}}$. La concentración de bulto del electrolito, $\rho$ es entonces dada por $\frac{N}{L_{\mathrm{b}}^{3}}$, donde $N$ es el número de iones positivos (o negativos). La mancuerna está hecha de dos iones fijos, dispuestos simétricamente a lo largo del eje que pasa por los dos centros de dos caras opuestas. La separación centrocentro de estos dos iones que forman la mancuerna es denotada por $\tau$. Un dispositivo similar fue también usado para estudiar dos macroiones fijos $[23,24,25]$. Se ha usado simulación de DME para calcular el movimiento de los iones móviles del fluido, acoplados con un baño térmico que actúa a través de una fuerza estocástica, $\mathbf{W}_{i}(t)$, la cual tiene un valor promedio igual a cero. La ecuación que descrite el movimiento de la i-ésima partícula es

$$
m \frac{d^{2} \mathbf{r}_{i}}{d t^{2}}=-\nabla_{i} U-m \Gamma \frac{d \mathbf{r}_{i}}{d t}+\mathbf{W}_{i}(t)
$$

donde $m$ denota la masa de la partícula, $\Gamma$ es el coeficiente de fricción y $-\nabla_{i} U$ es la fuerza, que tiene dos contribuciones: (i) la interacción de tipo Coulomb y (ii) la interacción por volumen excluido. La fricción y la fuerza estocástica están relacionadas a través del teorema de fluctuación disipación $\left\langle\mathbf{W}_{i}(t) \cdot \mathbf{W}_{j}\left(t^{\prime}\right)\right\rangle=6 m \Gamma k_{B} T \delta_{i j} \delta\left(t-t^{\prime}\right)$. La interacción por volumen excluido es modelado como un potencial de Lennard-Jones $(L J)$ puramente repulsivo

$$
U_{L J}(r)=\left\{\begin{array}{lll}
4 \epsilon_{L J}\left[\left(\frac{a}{r}\right)^{12}-\left(\frac{a}{r}\right)^{6}\right]+\epsilon_{L J} & \text { para } & \mathrm{r}<2^{1 / 6} \mathrm{a} \\
0 . & \text { para } & \mathrm{r} \geq 2^{1 / 6} \mathrm{a}
\end{array}\right.
$$

donde $a$ es el diámetro iónico. La interacción eleetrostática entre cualquier par de iones $i$ y $j$, donde $i$ y $j$ denotan a cualquier par de iones, ya sea un ion de la mancuerna o un ion del fluido, se escribe como

$$
U_{e l}(r)=k_{B} T \ell_{B} \frac{z^{2}}{r}
$$

donde $\ell_{B}=\frac{e^{2}}{\varepsilon k_{B} T}$ es la longitud de Bjerrum la cual describe la fuerza electrostática y $z$ es la valencia de la sal $\left(z_{i}=z_{j}=z\right)$. Para relacionar los cálculos de simulación con los de dinámica molecular hemos elegido el parámetro de la energía en! e! potencia! $L J$ como $\epsilon_{L J}=k_{B} T$ (dende $T=298 \mathrm{~K}$ ) y $a=4.25 \AA$. Esto nos da una lon ;itad de Bjerrum de $\ell_{B}=1.68 a=7.14 \AA$. Un sistema macroscópico es imitado al impor er conćiciones de contorno periódicas. Las interacciones coulómbicas de largo alcance fueron taatadas usando un método eficiente de sumas de Ewald optimizado, es decir, el métc do 'par' ic'eparticle-particle-mesh' $\left(\mathrm{P}^{3} \mathrm{M}\right)[74,75]$. Para evitar los efectos de borde de la caja st usó una caja de dimensión $L-b$ suficientemente grande, típicamente 10 veces (o en algunos 
casos más) la longitud de Debye-Hückel. El número de iones en la caja es de 500 en todos los casos (soluciones concentradas y diluidas). Es importante mencionar que el cálculo $g^{[3]}(r, \theta)$ es estadísticamente demandante de recursos de cómputo y especialmente para ángulos pequeños, dado que la cantidad de información varía como $\sin (\theta)$. El hecho de que el observable $g^{[3]}(r, \theta)$ involucra solamente a un elemento de ángulo sólido, reduce fuertemente la cantidad de información disponible, comparada con la función de correlación por pares, donde en este caso un ángulo sólido completo de $4 \pi$ está disponible. Para vencer esta dificultad hemos considerado un rango de ángulo, $\Delta \theta$ lo suficientemente grande, (típicamente entre $5-15^{\circ}$ dependiendo de la valencia y concentración), de tal forma que la información recopilada contenga la menor cantidad de ruido como sea posible. Por otro lado, $\Delta \theta$ no debe ser tan grande o de otra forma la resolución es muy baja. Por cada sistema en consideración debe encontrarse un compromiso entre estos dos efectos. Finalmente para el cálculo de la fuerza efectiva promedio entre dos iones consideramos el mismo sistema pero sin iones fijos presentes. Por lo tanto, calculamos el potencial de la fuerza promedio conociendo $g(r)$, y entonces por derivación la fuerza efectiva.

\subsection{Resultados}

Vamos a restringir nuestro estudio ya que presentar todos los casos, para todas las configuraciones de la mancuerna (separación $\tau$ y carga) así como los parámetros del electrolito (i. e. $\rho \mathrm{y} z$ ) no es el objetivo de este trabajo.

Hemos resuelto la Ec. (5.18) con valores de $T=298 \mathrm{~K}, \varepsilon=78.5$ y el diámetro iónico $a=4.25 \dot{\AA}$. En todos nuestros cálculos, las partículas de la mancuerna son de la misma especie que las del fluido. La distancia $r$ es medida desde el centro de la mancuerna y el ángulo $\theta$ está representado en la Fig. 5.1. En nuestra discusión adoptamos la siguiente convención: $g_{3 \gamma i}^{[3]}(r, \theta ; \tau)$ es denotada como $g_{++-}^{[3]}(r, \theta ; \tau)$ para la función de distribución de los iones negativos si la mancuerna está formada de dos iones positivos; $g_{+--}^{[3]}(r, \theta ; \tau)$ para la función de distribución de los iones negativos si la mancuerna está hecha de una partícula positiva (partícula de la especie $\beta$ ) y una partícula negativa (partícula de la especie $\gamma$ ). etc. En esta notación la partícula de la especie $\beta$ se localiza en $\theta=0$ y la partícula de la especie $\gamma$ en $\theta=\pi$. Por simetría las funciones de distribución satisfacen $y_{---}^{3}(r, \theta)=g_{-+}^{[3]}(r, \theta)$ y también $g_{+--}^{[3]}(r, \theta)=g_{+-+}^{[3]}(r, \pi-\theta)$.

\subsubsection{Electrolito monovalente}

En primer lugar presentamos el estudio de un electrolito monovalente concentrado $(\rho=$ 1.M). Aqui se muestra que TPE describe correctamente al MPR tan bien como las teorías estándar (HNC/MSA en este caso). Sin embargo, nuestro estudio muestra información de la función de distribución de tres partículas que es información no proporcionada por las teorias estándar. En nuestro estudio presentamos la gráfica tridimensional de algunas soluciones de la Ec. $(5.18)\left(g_{\gamma \beta i}^{[3]}(r, \theta)\right)$, así como su comparación con los resultados de 
dinámica molecular para ciertos casos. Adicionalmente, presentamos una comparación de la función de correlación entre pares obtenida por medio de TPE-HNC/MSA, la teoría estándar HNC/MSA y de simulación por DME.
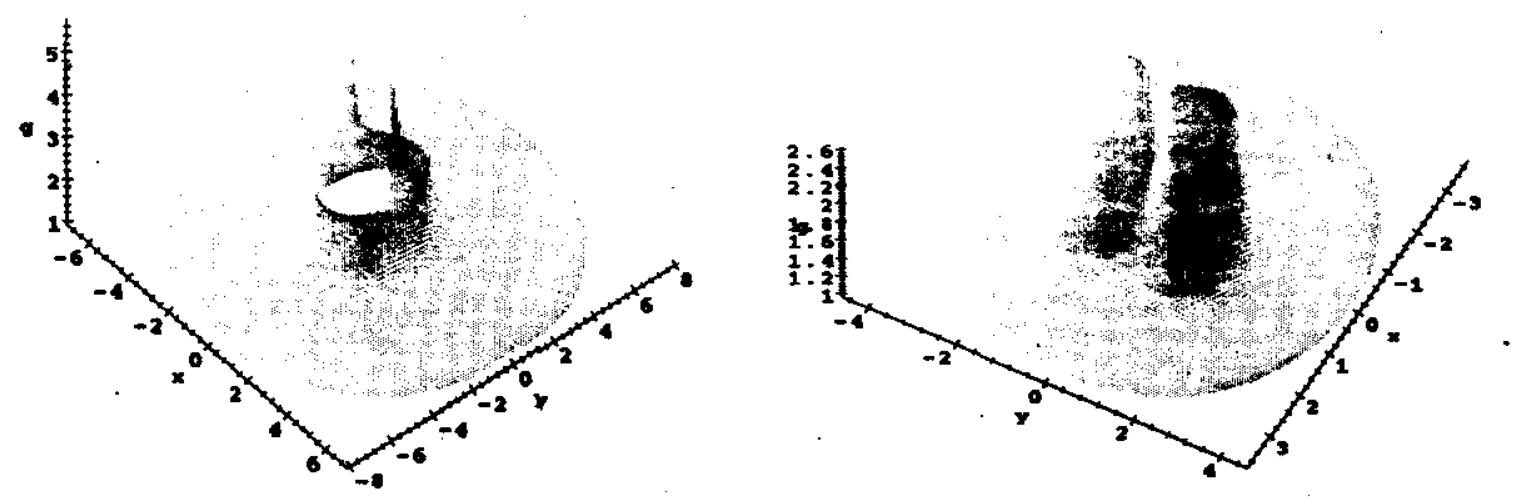

Figura 5.3: Gráfica tridimensional (en coordenadas cartesianas) de la función de distribución de tres partículas: (a) $g_{++-}^{[3]}(r, \theta ; \tau=a)$ y (b) $g_{++-}^{[3]}(r, \theta ; \tau=3 a)$. Los datos fueron obtenidos por medio de TPE-HNC/MSA para un electrolito monovalente a una concentración de $\rho=1 \mathrm{M}$.

La gráfica tridimensional de $g_{++-}^{[3]}(r, \theta ; \tau)$ obtenida de la solución de la Ec. (5.18) es mostrada en la Fig. 5.3. 'En la Fig. 5.3a mostramos el caso de $\tau=1.5 a$ en donde se encuentra una fuerte variación de la función en la región cercana a la mancuerna (en la superficie de máximo acercamiento). El máximo del valor de contacto de los perfiles de concentración está en $\theta=\pi / 2,3 \pi / 2$, mientras que el valor mínimo está en $\theta=0, \pi$. La gráfica de $g_{++-}^{[3]}(r, \theta ; \tau)$ correspondiente a una separación de $\tau=3 a$ se muestra en la Fig. 5.3b. Para este valor de $\tau$ tenemos una distribución más simétrica alrededor de cada partícula de la mancuerna.

En la Fig. 5.4 se muestra la comparación entre los resultados obtenidos por medio de TPE-HNC/MSA y DME, para dos casos de $g_{+--}^{[3]}\left(r, \gamma_{;} ;\right.$): (a) $\tau=a$ (b) $\tau=2 a$, con los siguientes parámetros del sistema $\rho=1 \mathrm{M}, z==$. La comparación se lleva a cabo en $\theta=\pi / 2^{1}$. Se observa que los perfiles de concen racion calculados con TPE muestran un muy buen acuerdo con los resultados de DME.

En la Fig. 5.5 se muestra la gráfica tridimensional d $g_{+--}^{[3]}(r, \theta ; \tau)$ obtenida por medio de TPE-HNC/MSA (Ec. (5.18)). En la Fig. 5.5. se rauestra el caso de $\tau=1.5 a$ mientras

\footnotetext{
${ }^{1} \mathrm{El}$ ángulo $\theta$ está referido respecto al centro de la mancuerna como se muestra en la Fig. 5.1
} 


$$
\rho=1.0 \mathrm{M}, \theta=90^{\circ}, \tau=a,++
$$

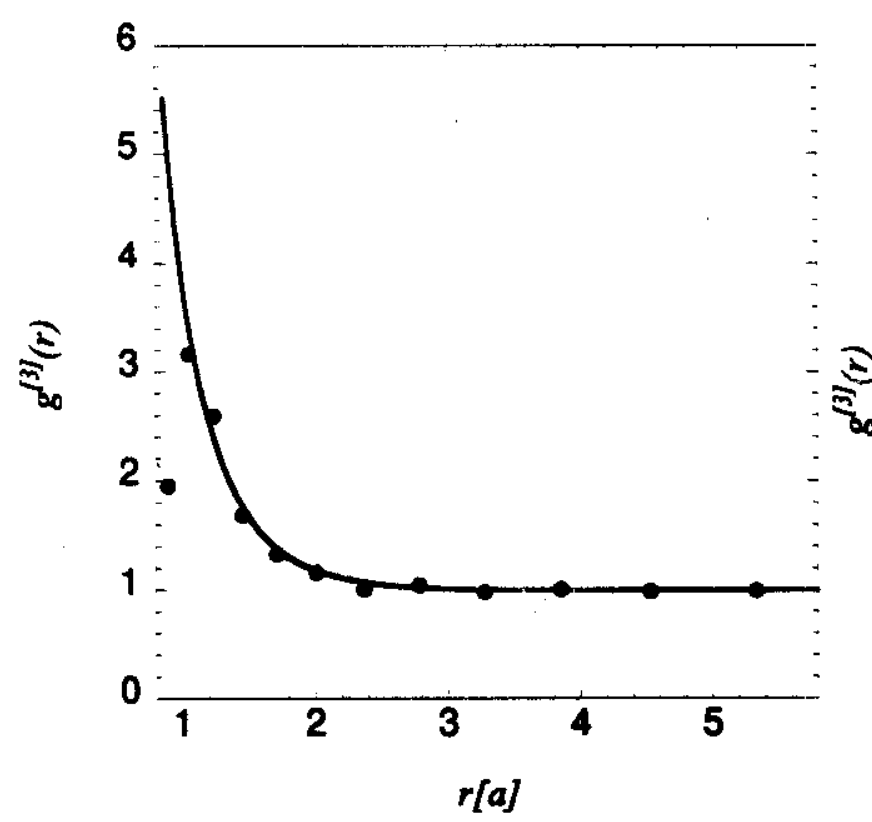

$p=1.0 \mathrm{M}, \theta=90^{\circ}, \tau=2 a,++$

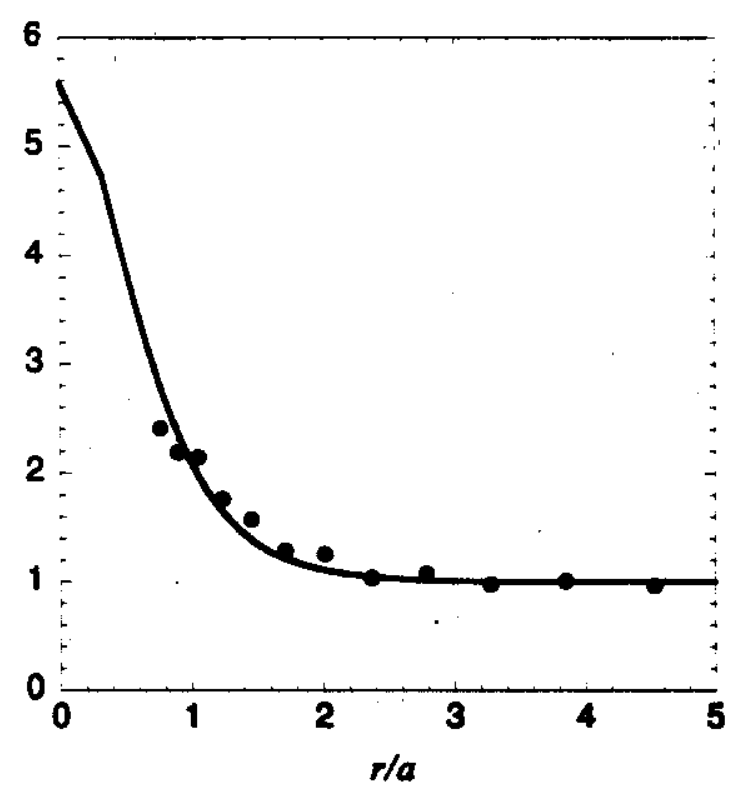

Figura 5.4: Función de correlación de tres partículas para una mancuerna hecha de dos iones . positivos monovalentes: (a) $g_{++-}^{[3]}(r, \theta=\pi / 2 ; \tau=a)$ y (b) $g_{++-}^{[3]}(r, \theta=\pi / 2 ; \tau=2 a)$. El fluido es un electrolito monovalente a una concentración de $\rho=1 \mathrm{M}$. Las líneas solidas representan los resultados de TPE-HNC/MSA mientras que los resultados de dinámica molecular se muestran en puntos negros.

que la gráfica tridimensional de $g_{+--}^{[3]}(r, \theta ; \tau)$ correspondiente a una separación de $\tau=3 a$ es mostrada en la Fig. 5.5b. En la Fig. 5.6 se muestra la comparación entre los resultados de $g_{+--}^{[3]}(r, \theta ; \tau)$ obtenidos por medio de TPE-HNC/MSA y DME, con los mismos parámetros del fluido que en la Fig. 5.5. La comparación con los datos de DME se lleva a cabo en $\theta=\pi / 4$. En esta figura se muestran los perfiles de concentración para (a) la especie cargada negativamente y (b) la especie cargada positivamente, los cuales muestran un acuerdo cualitativo excelente con los resultados de DME.

Finalmente, para mostrar que en estas condiríciones $(\rho=1 \mathrm{M}$ y $z=1)$ la descripción del MPR por medio de TPE es tan buena como la de las teorías estándar (por ejemplo HNC o BGY $[133,136])$, presentamos los resultados de la función de distribución por pares obtenida por medio de TPE. La conı ración de TPE con las teorias estándar debe hacerse a nivel de $g^{(2)}(r)$ ya que con estaś teoriás no es posible obtener $g^{[3]}(r)$. Por otra parte, es bien sabido que en estas condici,nts tanto HNC/MSA así como HNC describen correctamente al MPR. En la Fig. 5.7 se inuestran l:ss funciones de distribucion por pares (a) $g_{+-}(r)$ y (b) $g_{++}(r)$ como una funcion de $r$, park, un electrolito monovalente a una concentración de $\rho=1 \mathrm{M}$. Se observa que TPE y HNC 'MSA muestran muy buen acuerdo con los datos de simulación para ambos casis. 

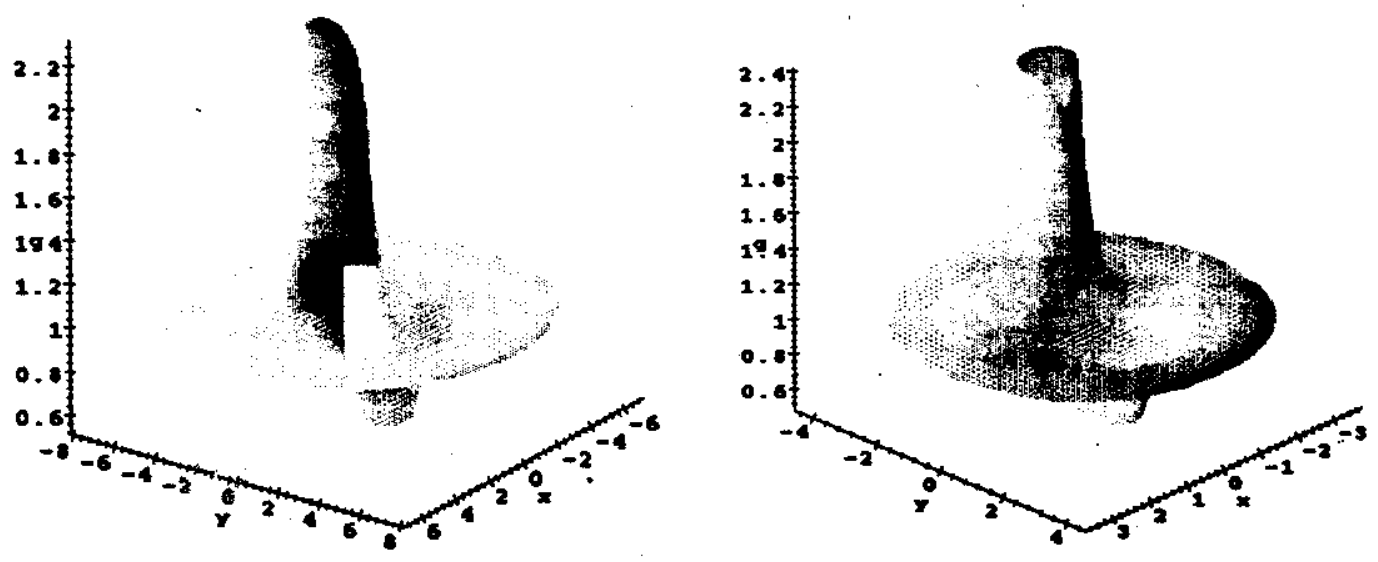

Figura 5.5: Gráfica tridimensional (en coordenadas cartesianas) de la función de distribución de tres partículas para una mancuerna hecha de dos iones monovalentes de signo opuesto: (a) $g_{+-}^{[3]}(r, \theta ; \tau=a)$ y (b) $g_{+--}^{[3]}(r, \theta ; \tau=3 a)$. Los datos fueron obtenidos por medio de TPE-HNC/MSA para un electrolito monovalente a una concentración de $\rho=1 \mathrm{M}$.

Con los resultados hasta aquí presentados podemos decir que TPE describe correctamente al electrolito monovalente bajo estas condiciones. Por otro lado, podemos tener confianza de nuestros resultados de $g^{[3]}(r)$. Sin embargo, para mostrar que el método es robusto se hacen pruebas más severas. El electrolito divalente representa una prueba más severa para cualquier teoría, por lo que a continuación mostramos los resultados de TPE obtenidos para el electrolito divalente. Como un caso particular, presentamos los resultados del electrolito diluido divalente en donde otras teorías fallan en su descripcion $[46,47]$. En nuestros resultados es interesante ver la predicción de TPE. 

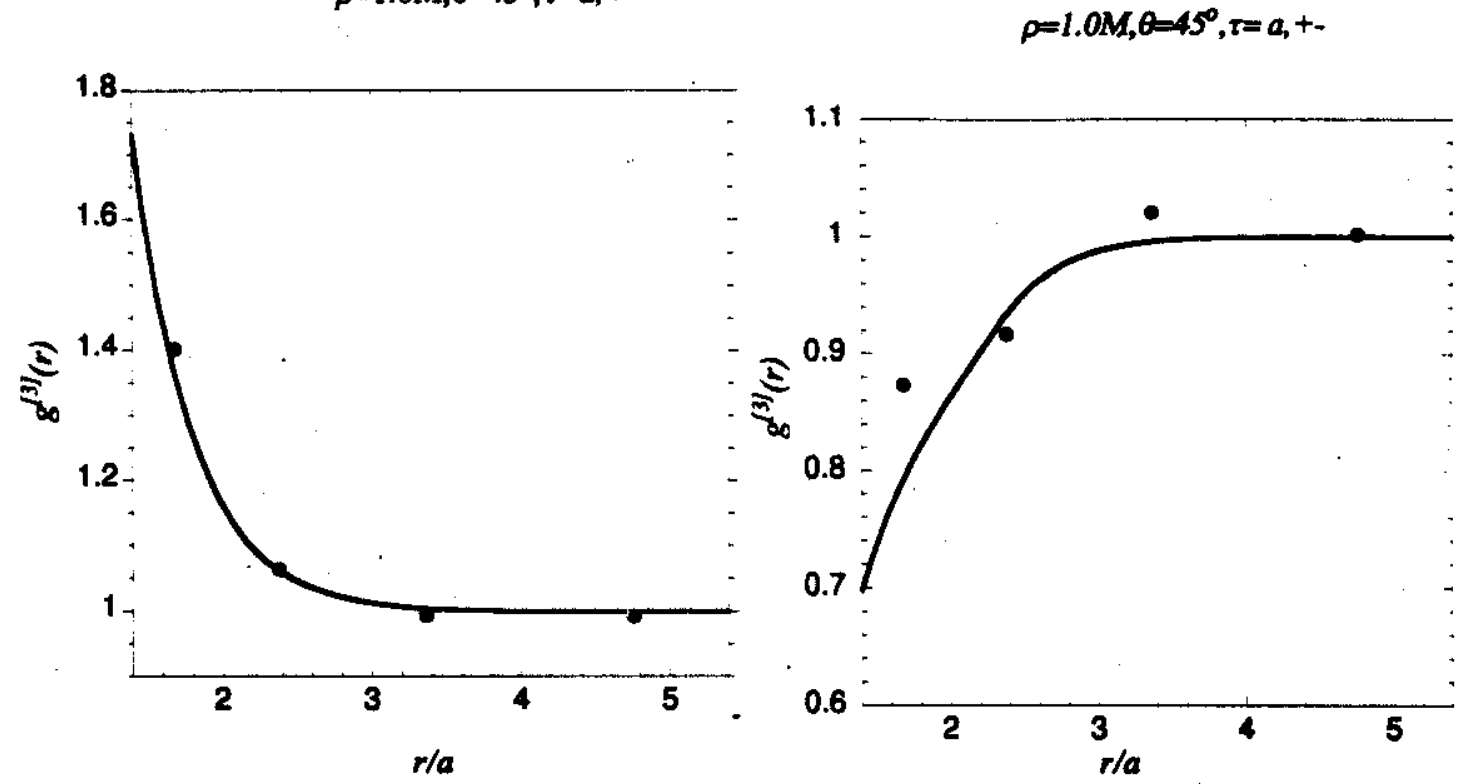

Figura 5.6: Función de correlación de tres partículas para una mancuerna hecha de dos iones monovalentes de signo opuesto (a) $g_{+--}^{[3]}(r, \theta=\pi / 4 ; \tau=a)$ y (b) $g_{+-+}^{[3]}(\tau, \theta=\pi / 4 ; \tau=$ a). El fluido es un electrolito monovalente a una concentración de $\rho=1 \mathrm{M}$. Las líneas sólidas representan los resultados de TPE-HNC/MSA, mientras que los resultados de dinámica molecular se muestran en puntos negros.

\subsubsection{Electrolito divalente}

A continuación presentamos un estudio del electrolito divalente el cual es relevante dado que las correlaciones, tanto de largo alcance como por volumen excluido, son importantes. En el caso divalente hemos considerado dos concentraciones típicas: (i) el caso concentrado con $\rho=1 \mathrm{M}$ y (ii) el caso diluido con $\rho=0.005 \mathrm{M}$. Hemos comparado sistemáticamente la teoría con los datos de simulación, para $g_{\beta \gamma i}^{[3]}(r, \theta ; \tau=a)$ (i. e., cuando los dos iones que forman la mancuerna están en contacto), para dos ángulos dados $\theta=\pi / 4$ y $\pi / 2$. También presentamos los perfiles $g_{\beta \gamma i}^{[3]}(r, \theta ; \tau)$ para diferentes configuraciones de la mancuerna, $\tau$. Finalmente presentamos la fuerza efectiva obtenida por medio de TPE-HNC/MSA, la teoría estándar HNC/MSA y de simulación por DME, la cual guarda una relación directa con la función de distribución por pares, $g_{i j}(r)$.

\section{Caso concentrado}

En esta sección tratamos con la solución electrolítica concentrada $(\rho=1 \mathrm{M})$. En estos sistemas, el apantallamiento electrostático muy alto. El estudio del sistema bajo estas condiciones es importante para probar la teoría TPE-HNC/MSA, dado que bajo estas condiciones las teorias para fluidos homogéneos e inhomogéneos normalmente producen 

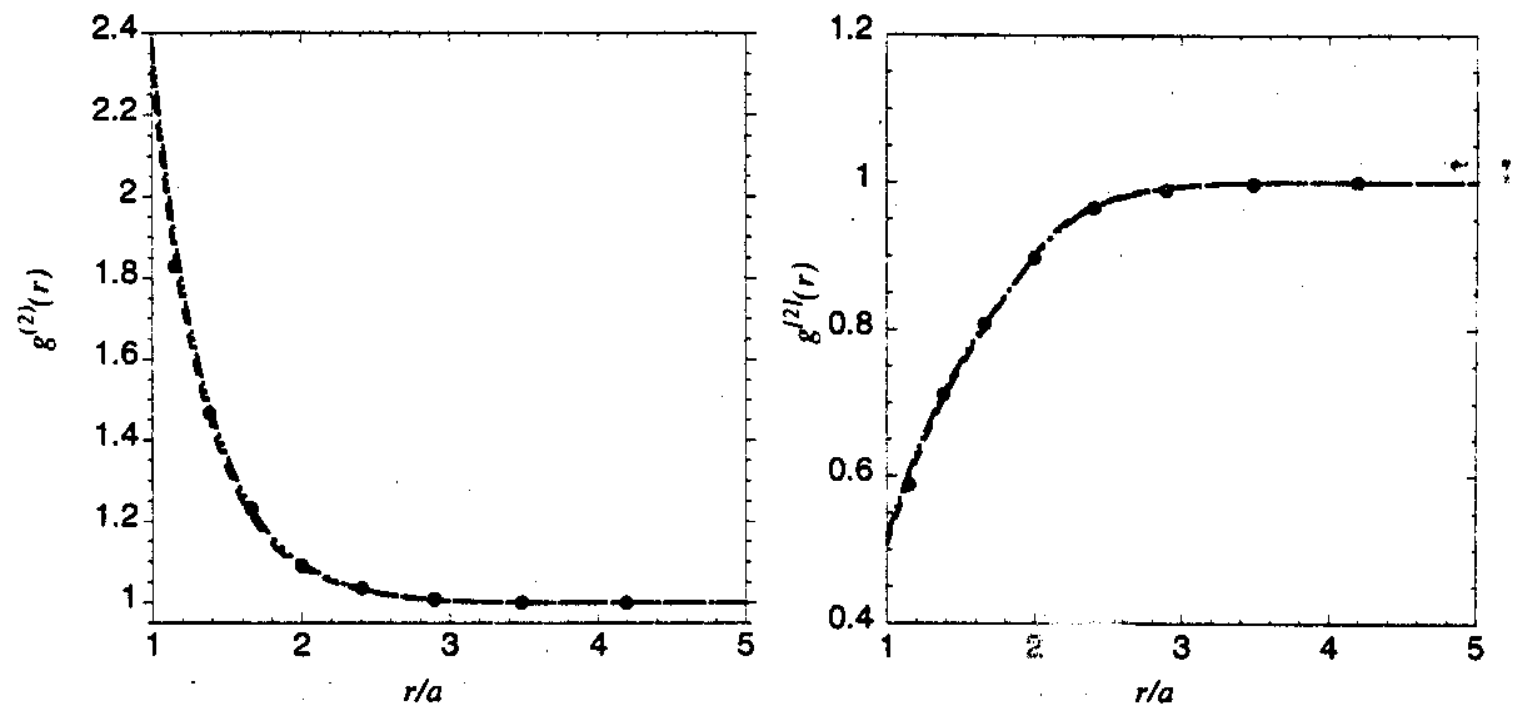

Figura 5.7: Funciones de distribución por pares (a) $g_{+-}(r)$ y (b) $g_{++}(r)$ como función de $r$, para un electrolito con los parámetros $\rho=1 \mathrm{M}$ y $z=1$. La línea sólida representa los resultados de TPE-HNC/MSA, la línea intermitente los resultados de la teoría estándar HNC/MSA. Los resultados de DME se muestran en puntos negros.

buen acuerdo con los datos de simulación molecular [46, 84].

\section{Mancuerna cargada simétricamente}

Primeramente consideramos una mancuerna simétrica hecha de iones positivos. En la Fig. 5.8 se muestran los resultados para la función de distribución de tres partículas $g_{++-}^{[3]}(r, \theta ; \tau)$ y $g_{+++}^{[3]}(r, \theta ; \tau)$. Los parámetros del sistema son $\rho=1 \mathrm{M}, z=2$ y $\tau=a$. La comparación con los datos de DME se lleva a cabo en $\theta=\pi / 2$. Los perfiles de concentración (calculados de TPE) para las especies del fluido cargadas negativamente ("contraiones"), muestran un acuerdo cuantitativo excelente con los resultados de simulación, aun para la distancias muy próximas a la distancia de máximo acercamiento de los iones con la mancuerna. Sin embargo, observamos pequeñas diferencias de los perfiles de concentración a la distancia de máximo acercamiento. Esto es debido al término de corto alcance usado para modelar el volumen excluido en ambas teorías, ya que en la simulación se usa un potencial repulsivo suave de tipo Lennard-Jones, mientras que en la teoría se usa un potencial repulsivo de esfera dura. En la comparación de los resultados para la especie cargada positivamente ("coiones"), también se tiene un acuerdo cualitativo excelente con los datos de DME. En todos los cálculos TPE y DME predicen oscilaciones en las funciones de distribución. El máximo de la función de distribución de los coiones es sobreestimado por TPE al compararlo con D.ME , aunque la localización del máximo es la mișma. Esto significa que TPE sobreestima ligeramente la concentración local de los contraiones.

Para $\theta=\pi / 4$ (ver Fig. 5.9) tenemos aún el mismo acuerdo cuantitativo que en la Fig. 5.8. Para los contraiones, se observa que el valor de $g_{++-}^{[3]}(r, \pi / 4 ; a)$ a la distar.- 


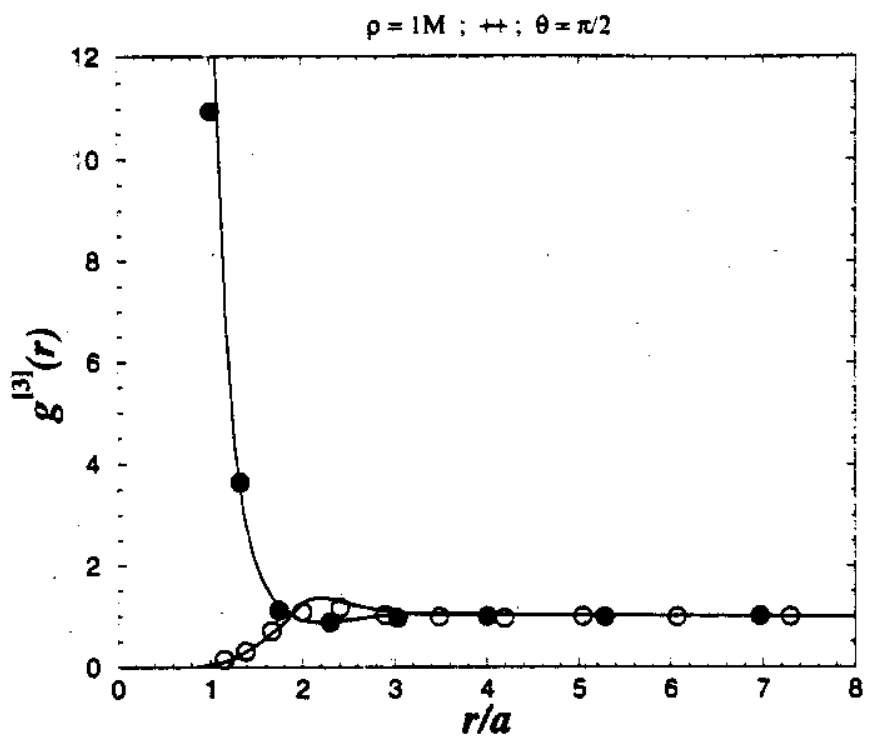

Figura 5.8: La función de correlación de tres partículas $g_{++i}^{[3]}(r, \theta=\pi / 2 ; \tau=a)$ para una mancuerna hecha de dos iones positivos divalentes. El fluido es un electrolito 1M. Las líneas sólidas representan los resultados de TPE-HNC/MSA. Los resultados de dinámica molecular se muestran en puntos negros.y blancos para $g_{++-}^{[3]}(r, \pi / 2 ; a)$ y $g_{+++}^{[3]}(r, \pi / 2 ; a)$, respectivamente.

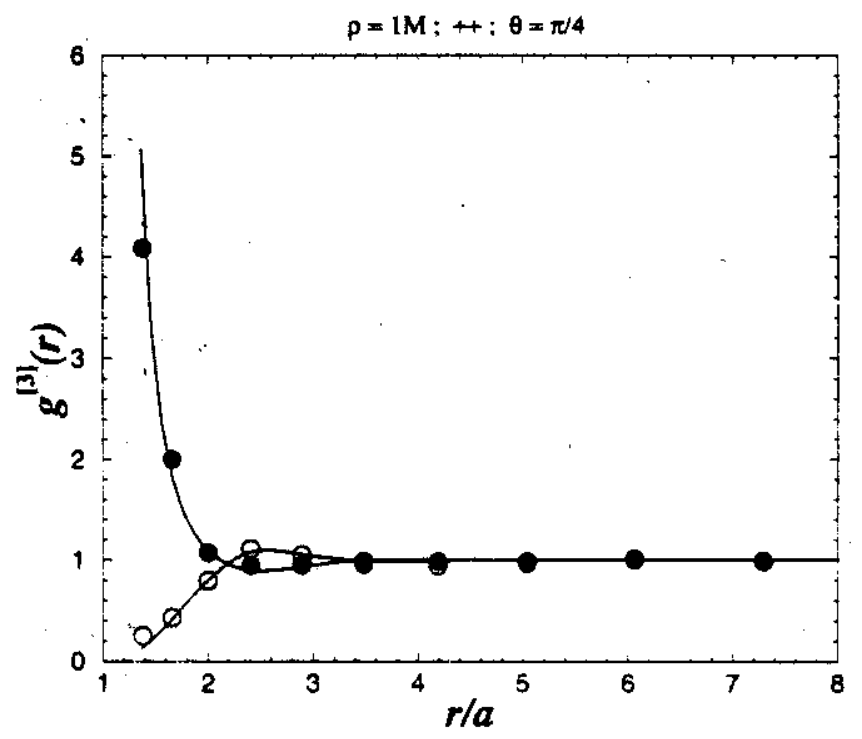

Figura 5.9: Los mismos cálculos que en la Figura 5.8 pero $\operatorname{coǹ} \theta=\pi / 4$.

cia de máximo acercamiento no es tan alto como en el caso de $\theta=\pi / 2$ (ver Fig. 5.8) para la gráfica correspondiente. Las razones físicas de estas características son directas y pueden explicarse en términos de argumentos electrostáticos. La distancia de máximo acercamiento de los iones con la mancuerna es mayor para $\theta=\pi / 4$ que para $\theta=\pi / 2$, 

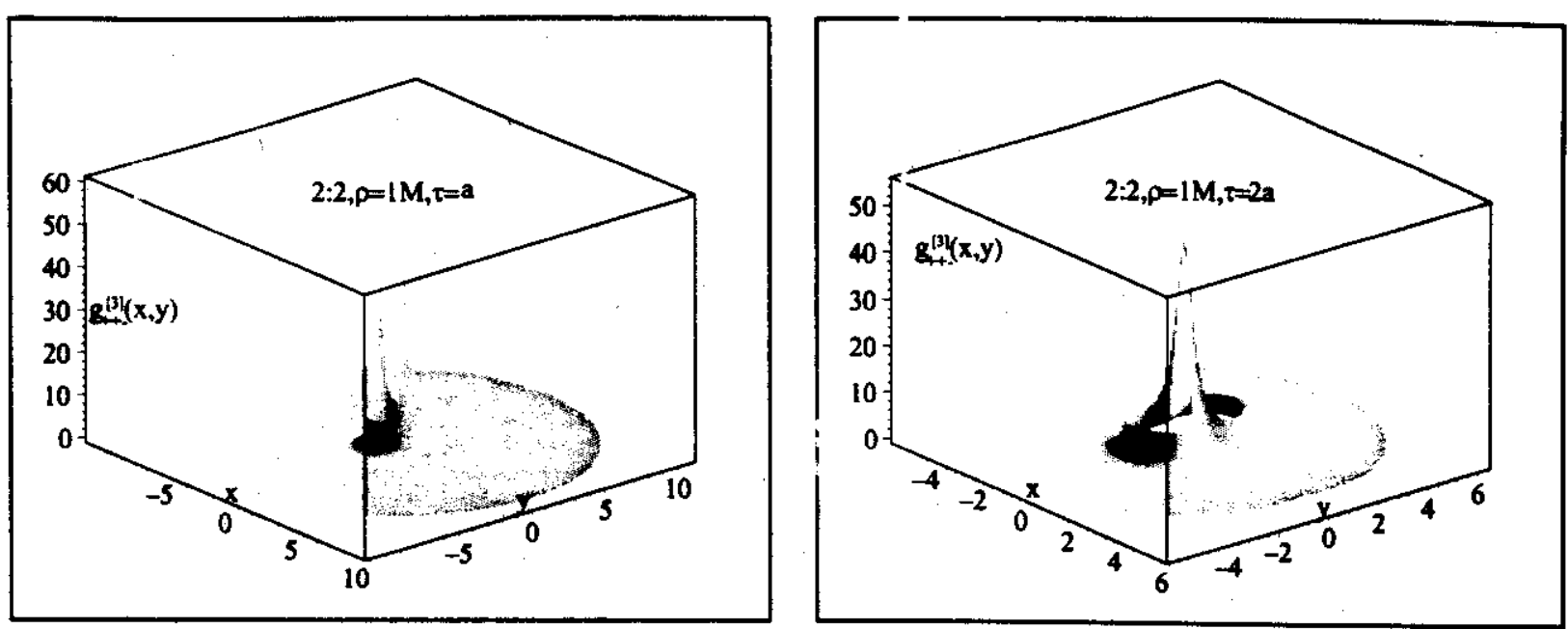

Figura 5.10: Gráfica tridimensional (en coordenadas cartesianas) de la función de distribución de tres partículas: (a) $g_{++-}^{[3]}(r, \theta ; \tau=a)$ y (b) $g_{++-}^{[3]}(r, \theta ; \tau=2 a)$, obtenida por TPE$\mathrm{HNC/MSA}$ para los mismos parametros del fluido que en las Figs. 5.8 y 5.9. El eje de la mancuerna es paralelo al eje $y$.

por lo tanto, la fuerza resultante entre la mancuerna y los coiones es mayor en $\theta=\pi / 2$. En el caso de los coiones tenemos ahora un excelente acuerdo cuantitativo entre los resultados de TPE y DME. La gráfica tridimensional (en coordenadas cartesianas) de la función de distribución de tres partículas (mancuerna-contraiones) $g_{++-}^{[3]}(r, \theta ; \tau)$, obtenida de la solución de TPE-HNC/MSA se muestra en la Fig. 5.10. Para $\tau=a$ (iones de la mancuerna en contacto), Fig.5.10(a) se muestra una variación fuerte en la función de distribución cercana a la mancuerna (en la superficie de máximo acercamiento). Como era de esperarse, el máximo del valor de contacto de los perfiles de concentración, es observado en $\theta=\pi / 2,3 \pi / 2\left(g_{++-}^{[3]} \approx 50\right)$, mientras que el valor mínimo está en $\theta=0, \pi\left(g_{++-}^{[3]} \approx 8\right)$. Por otra parte, se presentan oscilaciones en la función de distribución para cada valor del ángulo $\theta$. Hemos verificado esta característica y se mantiene para cada separación de la mancuerna, $\tau$. La gráfica tridimensional correspondiente a los coiones no es mostrada. Para la separación mayor de la mancuerna, $\tau=2 a$, [ver Fig.5.10(b)] la función de distribución presenta aún un pico muy alto en $\theta=\pi / 2$ y tiene un punto máximo en el punto medio de la mancuerna. Para una separación de la mancuerna lo suficientemente grande - enemos una distribución más simétrica alrededor de cada partícula de la mancuerna (no nostrada aquí).

\section{Mancuerna cargada asimétricamente}

Ahora consideramos aquí el caso antisimétrico, donde la mancuerna está formada de iones divalentes de signo opuesto. Los resultados para la función de distribuciones de tres partículas $g_{+--}^{[3]}(r, \pi / 2 ; a)$ y $g_{+-+}^{[3]}(r, \pi / 2 ; a)$ son mostrados en la Fig.5.11. En este caso, debido a la simetría del problema sucede que $g_{+--}^{[3]}(r, \pi / 2 ; a)=g_{+-+}^{[3]}(r, \pi / 2 ; a)$. De esta forma la función de distribución de tres partículas es idéntica tanto para las partículas positivas como para las partículas negativas. Dado que el campo eléctrico producido por 


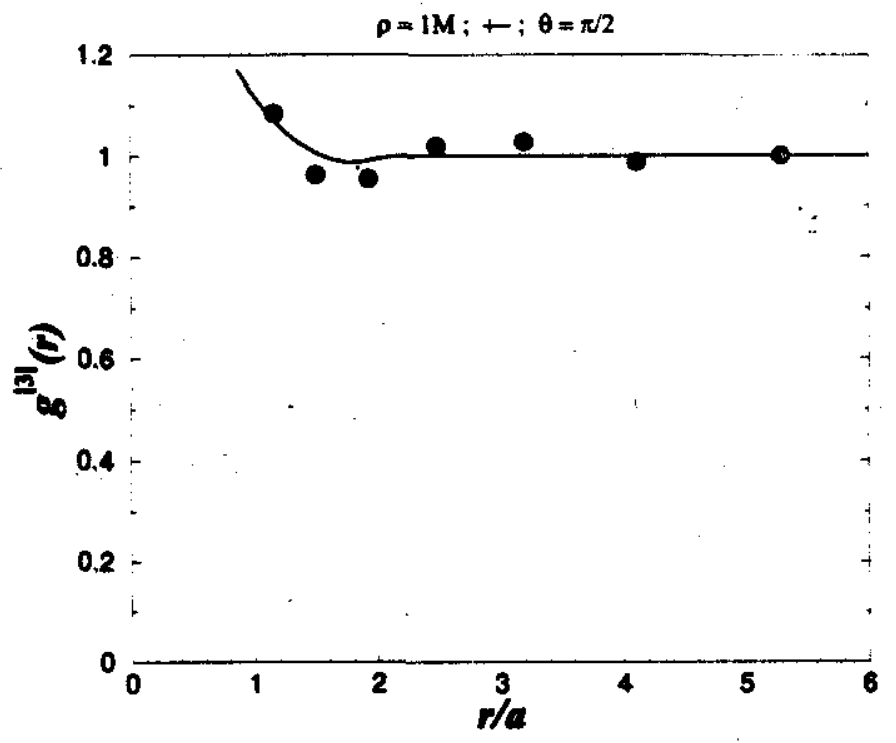

Figura 5.11: Función de distribución de tres partículas $g_{+\rightarrow i}^{[3]}(r, \theta=\pi / 2 ; \tau=a)$ para una mancuerna hecha de un ion positivo y un ion negativo, ambos divalentes. El fluido es un electrolito $1 \mathrm{M}$ divalente. La línea sólida representa los resultados de TPE-HNC/MSA mientras que la línea intermitente son los resultados de ASK. Los resultados de DME los mostramos en puntos negros. 


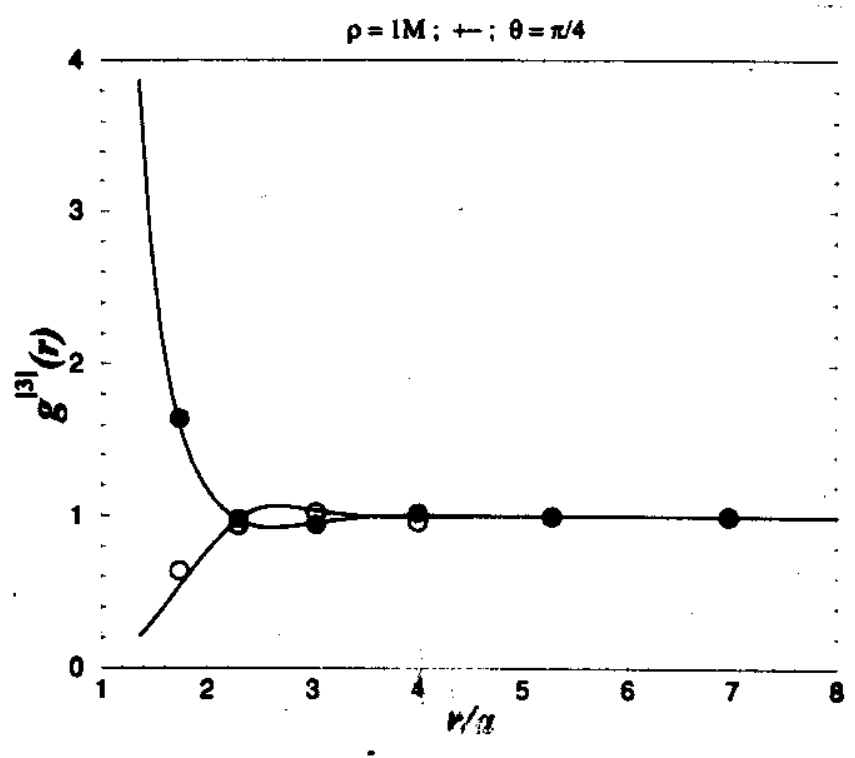

Figura 5.12: Los mismos cálculos que en la Fig. 5.11 pero $\operatorname{con} \theta=\pi / 4$. La líneas sollidas representan los resultados de TPE-HNC/MSA. Los resultados de DME se muestran en puntos negros y blancos para $g_{+--}^{[3]}(r, \pi / 4 ; a)$ y $g_{+-+}^{[3]}(r, \pi / 4 ; a)$, respectivamente.

la mancuerna en el eje donde $\theta=\pi / 2$ es cero, las correlaciones eléctricas son debidas únicamente a los iones del fluido. Consecuentemente, dado que el presente sistema se encuentra altamente apantallado, esperamos un comportamiento cuasineutral del fluido, que es lo que se muestra en la Fig. 5.11 para la teoría y simulación. Sin embargo, DME y TPE sugieren una ligera atracción cerca del punto de contacto. Este efecto puede ser explicado bajo argumentos electrostáticos simples. El mecanismo básico es la atracción electrostática debida a las cargas de signo opuesto localizadas arriba y abajo sobre el eje de la mancuerna. El hecho de que se presenten oscilaciones se debe principalmente a los efectos de volumen excluido.

Los resultados para $\theta=\pi / 4$ se muestran en la Fig. 5.12. Tenemos un acuerdo cualitativo entre teoría y los resultados de simulación, y esto se cumple para las dos especies iónicas.

La gráfica tridimensional en coordenadas cartesianas de la función de distribución de tres partículas $g_{+--}^{[3]}(r, \theta ; \tau=a)=g_{+-+}^{[3]}(r, \pi-\theta ; \tau=a)$, obtenida por TPE-HNC/MSA, es mostrada en la Fig. 5.13. El máximo y mínimo sobre la superficie de contacto de la mancuerna están localizados en $\theta=0$ y $\theta=\pi$, respectivamente. Se observan otra vez oscilaciones para cada ángulo así como para cada separación de la mancuerna, $\tau$.

\section{Fuerza efectiva}

La fuerza efectiva entre dos iones igualmente cargados y dos iones de carga opuesta como una función de su separación, $r$, está dada en la Fig. 5.14. Se observa que TPE y HNC/MSA muestran muy buen acuerdo con los datos de sinulación para ambos casos 


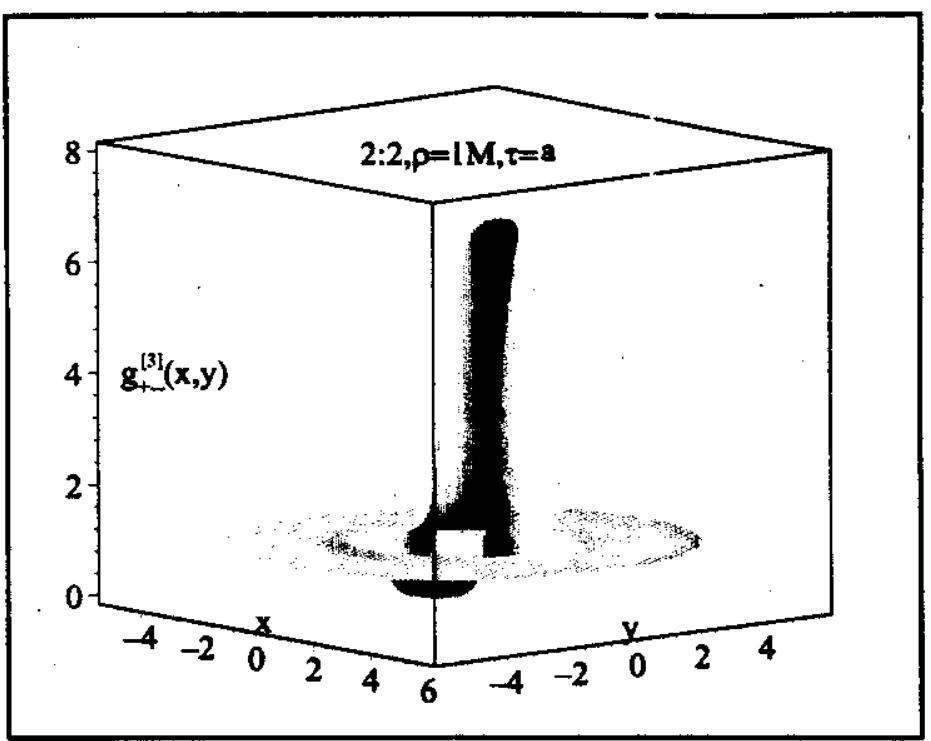

Figura 5.13: Grafica tridimensional (en coordenadas cartesianas) de la función de distribución de tres partículas, $g_{+--}^{[3]}(r, \theta ; \tau=a)$, obtenida por TPE-HNC/MSA, para los mismos parámetros del fluido que en las Figs. 5.11 y 5.12.

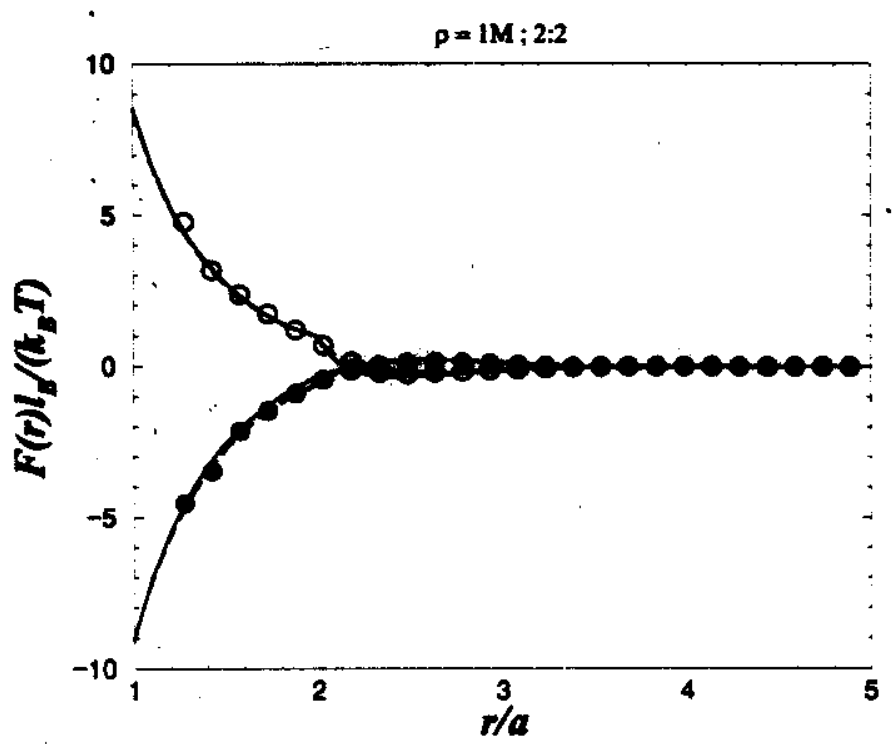

Figura 5.14: Fuerza media efectiva entre dos iones con la misma carga $\left(F_{++}\right)$y dos iones con carga opuesta $\left(F_{+-}\right)$como una función de su separación $r$, para el caso concentrado $(\rho=1 \mathrm{M}$ y $z=2$ ). La línea sólida representa los resultados de TPE-HNC/MSA, la línea intermitente. los resultados de la teoría estándar HNC/MSA. Los resultados de DME se muestran en puntos blancos y negros para $F_{+-}(r)$ y $F_{++}(r)$, respectivarnente. 
$\left(\mathrm{F}_{++} \mathrm{y} \mathrm{F}_{+-}\right)$, como era de esperarse.

Un efecto interesante es el camtio abrupto en la derivada de $\mathrm{F}_{++}(r)$ el cual ocurre en $r=2 a$, y que aparece menos marcado en los resultados de simulación (debido a la suavidad del potencial de interacción y también a la menor resolución de estos datos). Este cambio abrupto en la derivada no es un artificio de la teoría (o de la simulación) sino que es un efecto real. Este efecto sf debe a las correlaciones por volumen excluido y en menor grado a las correlaciones electrostáticas. Es claro que en $r=2 a$, la configuración con contraiones presentes exactamente entre dos coiones es energéticamente favorable (i. e., +-+ ). Cuando $r>2 a$ (más precisamente $r \rightarrow 2 a^{+}$) la presencia de un ion entre las partículas conduce a una mayor resistencia, al nivel de la fuerza de deplesion, cuando los dos iones se aproximan. Por otro lado, cuando $r>2 a$ (más precisamente $r \rightarrow 2 a^{-}$), la ausencia de un ion entre las partículas produce una menor resistencia a la aproximación entre los iones. Este mecanismo explica (i) la discontinuidad de $\mathrm{F}_{++}$en $r=2 a$ y (ii) el hecho de que $\left|\mathrm{F}_{++}^{\prime}\left(r \rightarrow 2 a^{-}\right)\right|<\left|\mathrm{F}_{++}^{\prime}\left(r \rightarrow 2 a^{+}\right)\right|$. Este efecto también podría ser observado en esferas duras neutras.

Hasta donde podemos ver, para $\mathrm{F}_{+-}$la discontinuidad en la derivada está ausente o prácticamente indetectable. Esto se debe al hecho de que, en $r=2 a$, la probabilidad de encontrar un ion entre dos iones de signo contrario (i. e., +-+ ) es considerable mẹnor a la obtenida con la configuración +-+ , además de que varía más suavemente.

\section{Caso diluido}
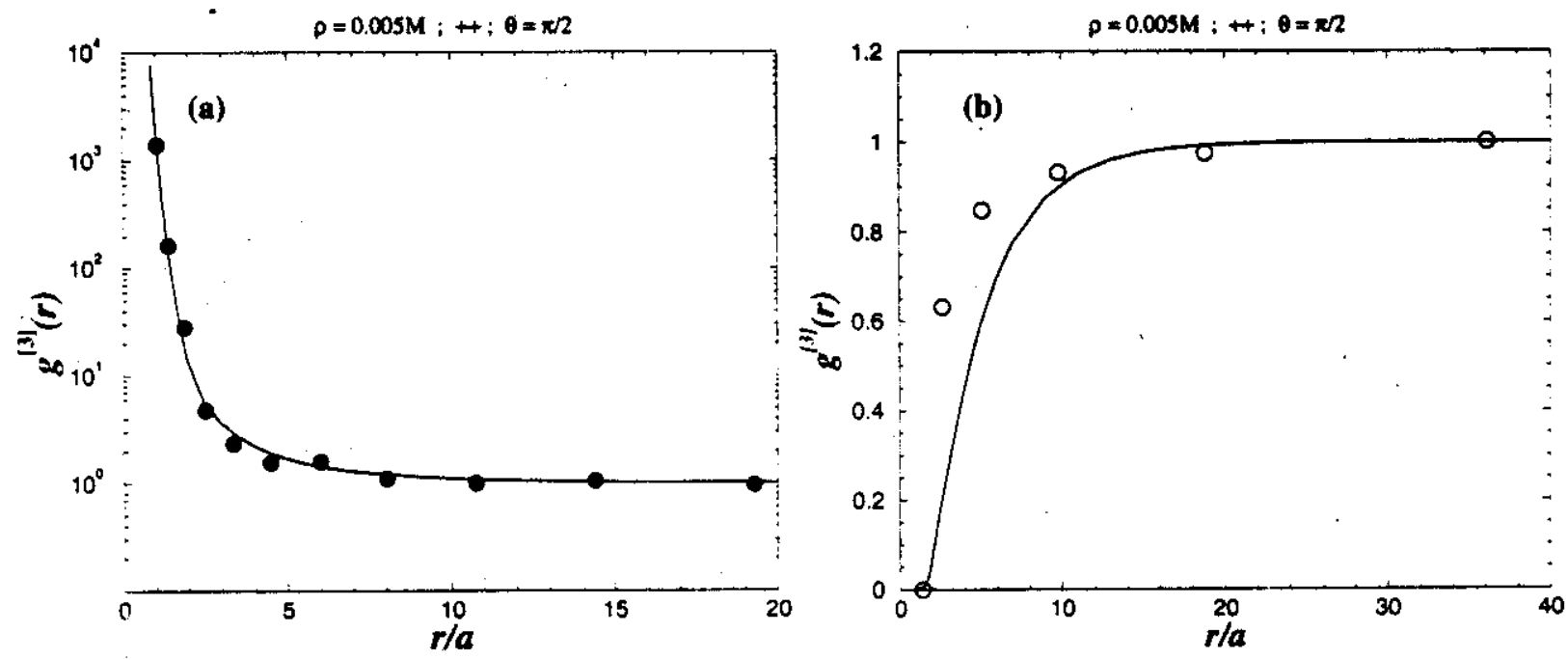

Figura 5.15: Función de correlación de tres partículas: (a) $g_{++-}^{[3]}(r, \pi / 2 ; a)$ y (b) $g_{+++}^{[3]}(r, \pi / 2 ; a)$. El fluido es un electrolito divalente a una concentración de $0.005 \mathrm{M}$. La linea sólida representa los resultados de TPE-HNC/MSA. Los resultados de DME se muestran en puntos negros y blancos para $(a) g_{+--}^{[3]}(r, \pi / 2 ; a)$ y $(b) g_{+-+}^{[3]}(r, \pi / 2 ; a)$ respectivamente. 
En esta sección tratamos con el caso diluido $(\rho=0.005 \mathrm{M})$ de iones divalentes $(z=$ 2). En soluciones a baja concentración, las correlaciones de largo alcance son las más relevantes. Aquí, la mayoría de las teorías de líquiàos fallan en la descripción de su comportamiento bajo estas condiciones [47, 84]. Por lo tanto, el estudio de soluciones electrolíticas a baja concentración de iones polivalentes representa una prueba fuerte para cualquier teoría de líquidos.

Mancuerna cargada simétricamente

Las Figs. 5.15(a) y 5.15(b) muestran la comparación entre los resultados de TPE $\mathrm{y}$ los datos de DME, para la función de correlación de tres partículas $g_{++-}^{[3]}(r, \pi / 2 ; a) \mathrm{y}$ $g_{+++}^{[3]}(r, \pi / 2 ; a)$, i. e., los contraiones y coiones de la mancuerna, respectivamente. Primero que todo, podemos ver que la DCE es más ancha que en el caso concentrado i. e., las correlaciones en este caso son de largo alcance. a los contraiones [ver la Fig. 5.15(a)], los resultados de TPE y DME muestran un muy buen acuerdo cualitativo, aun para puntos cercanos a la distancia de máximo acercamiento. Para el caso de los coiones (ver Figura 5.15(b)), i. e., $g_{+++}^{[3]}(r, \theta=\pi / 2)$, se encuentra un buen acuerdo cualitativo de TPE con los resultados de DME.

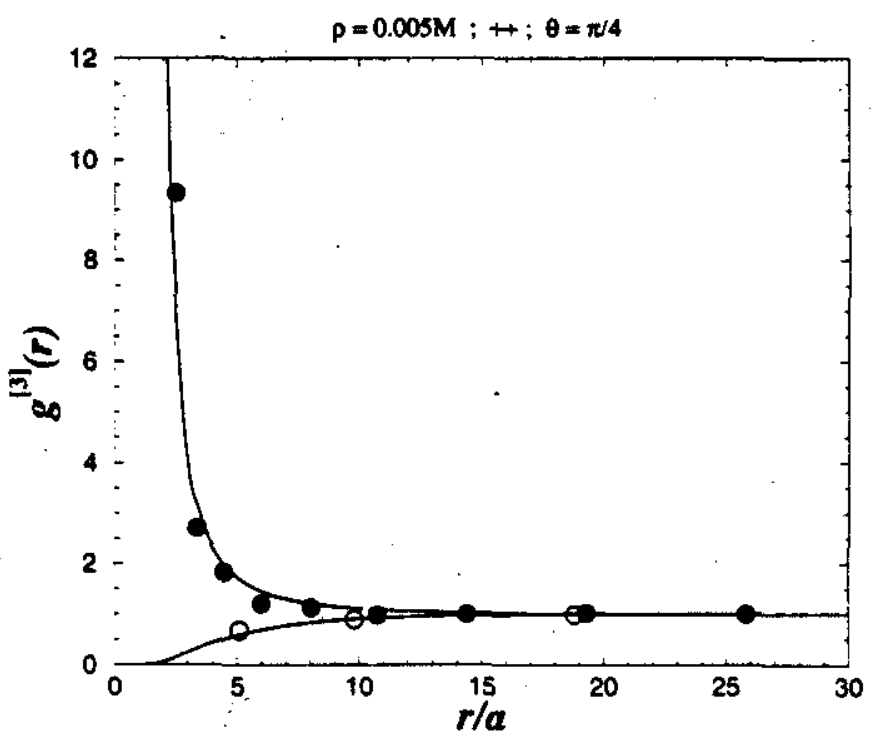

Figura 5.16: Los mismos cálculos que en la Figura 5.15 pero para $\theta=\pi / 4$.

an

En la Fig. 5.16, se muestra la comparación entre los resultados de TPE y DME, de la función de distribución de tres partículas para $\theta=\pi / 4$, para los mismos parámetros que en la Fig. 5.15. Se observa que el valor de contacto de $g_{++-}^{[3]}$ es mucho menor (por dos órdenes de magnitud) que para $\pi / 2$. Esto se debe a la disminución del campo eléctrico eîectivo en $\theta=\pi / 4$ respecto a $\theta=\pi / 2$. Como podemos ver, en $\theta=\pi / 4$, el acuerdo entre TPE y MD es cuantitativo y cualitativo. 

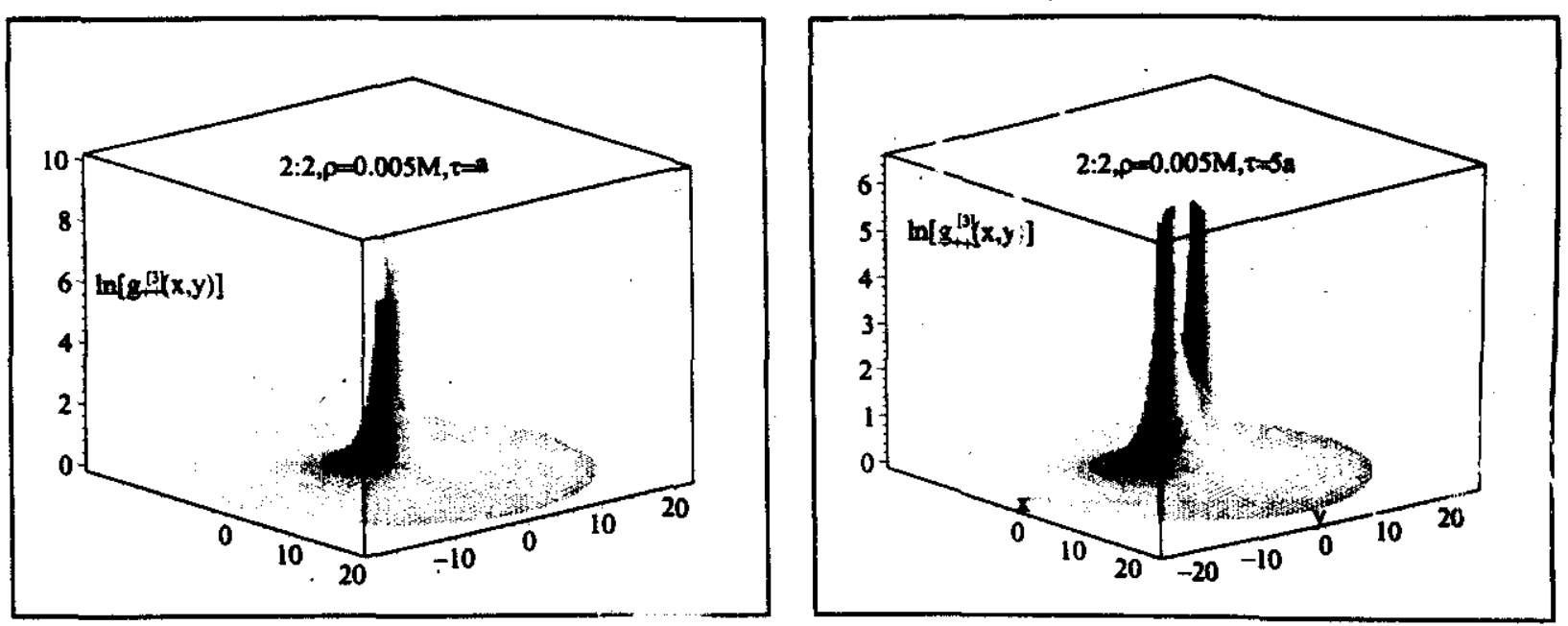

Figura 5.17: Gráfica tridimensional (en coordenadas cartesianas) del logaritmo de la función de distribución de tres partículas $g_{++-}^{[3]}(r, \theta ; \tau)$, obtenido por TPE-HNC/MSA para los mismos parámetros del fluido que en las Figs. 5.15 y 5.16. (a) $\tau=a$ (b) $\tau=5 a$.

En la Figura 5.17, se muestra la gráfica tridimensional, en coordenadas cartesianas, del logaritmo de la función de distribución de tres partículas (para los contráiones de la mancuerna), obtenida por TPE-HNC/MSA. Para $\tau=a$ [ver Fig. 5.17(a)], se observa una fuerte variación de la función de distribución en los puntos cercanos a la mancuerna, i. e., en la superficie de máximo acercamiento. Como es de esperarse el máximo de la función se observa en $\theta=\pi / 2$. En la Fig. 5.17(b) se muestra la grafica tridimensional del logaritmo de la función de distribución de tres partículas, para una separación de $\tau=5 a$. La función de distribución no muestra picos tan pronunciados como en el caso de la Fig. 5.17(a); en este sentido, ésta es más simétrica alrededor de cada ion que forma la mancuerna. Sin embargo, se observa que bajo estas condiciones $(\rho, z$ y $\tau)$ los iones que forman la mancuerna se encuentran todavía fuertemente correlacionados, i. e., sus dobles capas eléctricas se encuentran fuertemente traslapadas, aún para separaciones mayores. Bajo estas condiciones tuvimos que ir hasta una separación de $\tau=60 a$, la cual no es mostrada, para alcanzar a descorrelacionarlos.

Mancuerna cargada asimétrica

Por último, analizamos la función de distribución de tres partículas para el caso diluido, donde la mancuerna se encuentra constituida de do $s$ ones divalentes de carga opuesta. La Figura 5.18a muestra los resultados de TPE y DME. La comparación se lleva a cabo para los mismos parámetros del fluido que un las Figs. 5.15 y 5.16. En este caso, únicamente mostramos la comparación en $\theta=\pi / 4$, larto que para $\theta=\pi / 2$, tenemos que $g_{+--}^{[3]}(r, \theta=\pi / 2)=g_{+-+}^{[3]}(r, \theta=\pi / 2)=1$. Si: cbserva que el acuerdo entre TPE y DME es tan bueno como en el caso de la F.g. 5.16. En la Fig. 5.18b se muestra la gráfica tridimensional del logaritmo de la fun ción de distribución de tres partículas, 

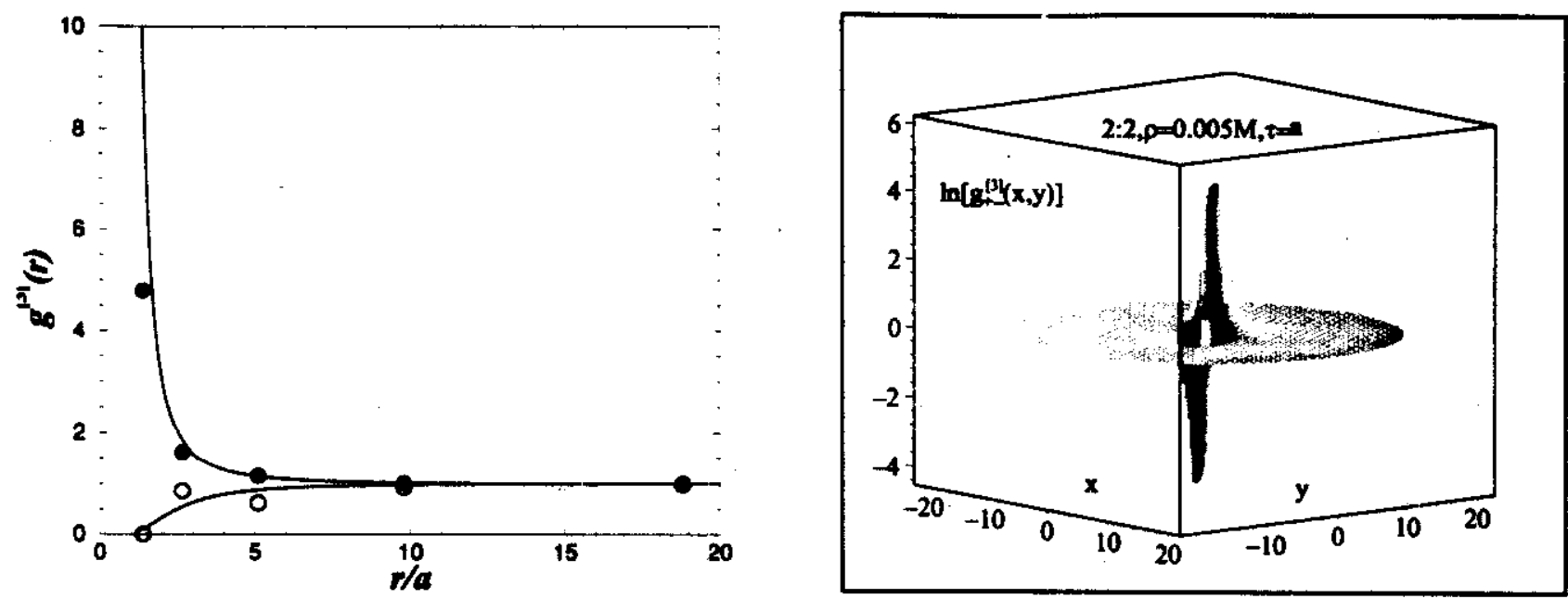

Figura 5:18: (a)Función de distribución de tres partículas; $g_{+-i}^{[3]}(r, \pi / 4 ; a)$. El fluido es un electrolito divalente a una concentración de $0.005 \mathrm{M}$. La línea sólida representa los resultados de TPE-HNC/MSA, mientras que la línea intermitente los de ASK. Los resultados de DME se muestran en puntos negros y blancos para $g_{+--}^{[3]}(r, \pi / 4 ; a)$ y $g_{+-+}^{[3]}(r, \pi / 4 ; a)$, respectivamente. (b)Gráfica tridimensional (en coordenadas cartesianas) de la función de distribución de tres partículas $g_{+--}^{[3]}(r, \theta)$, obtenido por TPE-HNC/MSA para los mismos parámetros del fluido que en (a).

$g_{+--}^{[3]}(r, \theta ; \tau=a)=g_{+-+}^{[3]}(r, \pi-\theta ; \tau=a)$, obtenida de TPE-HNC/MSA. Se observa que la gráfica de la función de distribución es centro-simétrica con respecto al eje de la mancuerna. Este hecho es consistente con el hecho de que $\ln \left[g^{[3]}(r)\right]$ es el potencial de la fuerza promedio. Como era de esperarse la función es fuertemente pronunciada en $\boldsymbol{\theta}=0$.

Fuerza efectiva

En la Fig. 5.19 está dada la fuerza efectiva entre dos iones divalentes, igualmente cargados $\left[F_{++}(r)\right]$ y con carga opuesta $\left[F_{+-}(r)\right]$, como una función de su separación $r$, cuando se encuentran inmersos en un electrolito divalente, a una concentración de $0.005 \mathrm{M}$. $\mathrm{Al}$ igual que en el caso concentrado se observa un cambio abrupto en la pendiente de $F_{++}(r)$ en $r=2 a$. El mismo mecanismo propuesto para el caso concentrado aplica en este caso. Este efecto importante no es predicho por la teoría HNC/MSA, probando así la mejora cualitativa al usar TPE. Esta mejor descripción se debe a la inclusión propia de correlaciones de largo alcance. Finalmente, observamos un acuerdo cualitativo entre los resultados de TPE y DME el cual es el resultado principal de este trabajo.

\subsection{Conclusiones de capítulo}

En este capítulo hemos investigado la estructura de un electrolito tipo MPR monovalente y divalente por medio de teorias de ecuaciones integrales y DME. Usando la extension a tres puntos de la ecuación integral HNC/MSA hemos obtenido la función de distribución 


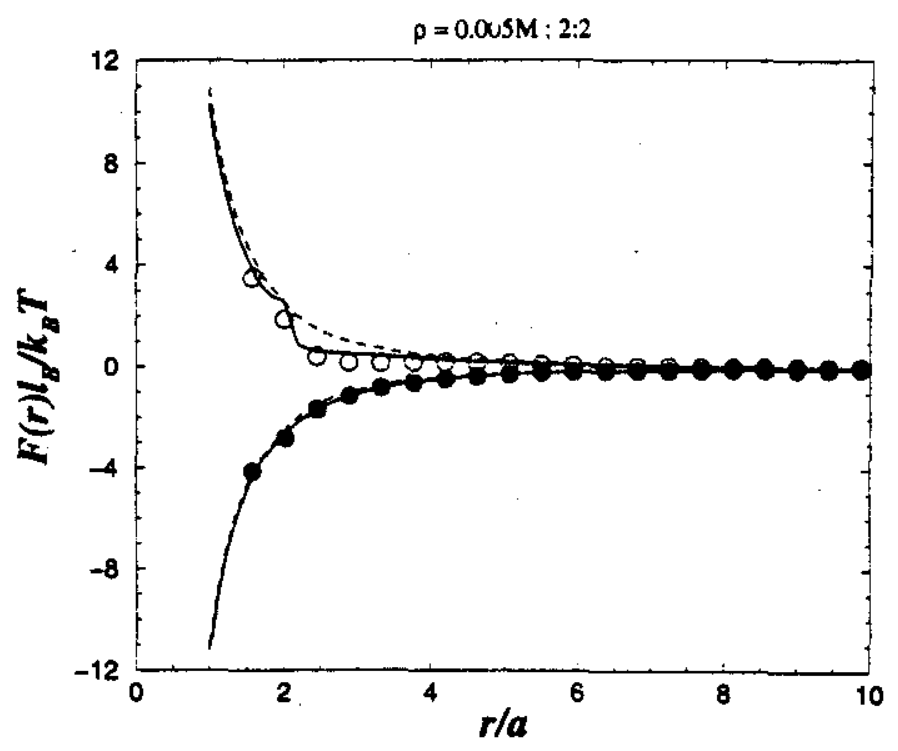

Figura 5.19: Fuerza efectiva entre dos iones igualmente cargados $\left(F_{++}\right)$y entre dos con carga opuesta $\left(F_{+-}\right)$como una función de su separación $r$ para el caso diluido $\rho=0.005 \mathrm{M}$ y $z=2$. La línea sólida representa los resultados de TPE-HNC/MSA, la línea intermitente representa los resultados de HNC/MSA. Los resultados de DME son mostrados en puntos negros y blancos para $F_{+-}(r)$ y $F_{++}(r)$, respectivamente.

de tres partículas, $g_{\gamma \beta i}^{[3]}(r, \theta)$, bajo ciertas condiciones, la cual ha sido comparada con datos de DME. Para el electrolito 1:1 el acuerdo cuantitativo entre DME y TPE es excelente tanto a nivel de $g^{[3]}(r, \theta)$ así como de $g^{(2)}(r)$. Por otro lado, para un electrolito divalente explícitamente reportamos resultados para dos concentraciones típicas del electrolito, $\rho$ : (i) el caso concentrado (1M) y (ii) el caso diluido $(0.005 \mathrm{M})$.

En lo que al caso concentrado se refiere, se encontró que $g_{\gamma \beta i}^{[3]}(r, \theta)$ siempre presenta oscilaciones. Por otro lado, la comparación detallada entre TPE y MD, la cual se llevó a cabo para $\tau=a$, muestra un excelente acuerdo tanto cualitativo como cuantitativo. Aunque no es mostrado, esto es cierto para cada valor de $\tau$.

Además de la función de distribución de tres partículas, $g_{\gamma \beta i}^{[3]}(r, \theta)$, investigamos otro observable, es decir, la fuerza media entre las partículas que forman la mancuerna como función de su separación. Hemos comparado la fuerza obtenida por DME con los resultados obtenidos por la teoría estándar HNC/MSA y TPE-HNC/MSA, para las mismas condiciones del electrolito.

En el estudio de la fuerza efectiva en el caso concentrado encontramos que, ambos, TPE y HNC/MSA tienen un excelente acuerdo con DME, lo cual es consistente con comparaciones previas entre HNC/MSA y Monte Carlo. Por lo que podemos concluir que TPE también es adecuado para describir soliciones electrolíticas concentradas. Es importante mencionar el comportamiento particular en la fuerza entre iones igualmente cargados observado en $r=2 a$, donde se observa un cambio abrupto de pendiente que 
se encuentra en ambas predicciones, TPE : HNC/MSA, el cual aparece suavizado por las simulaciones de DME. Este comportamiento no puede ser observado en la función de distribución por pares.

En el régimen diluido, el análisis de la función de distribución de tres partículas y de la fuerza efectiva promedio, muestra la naturaleza de largo alcance de las correlaciones. Para la función de distribución de tres partículas tuv mos que ir a una separación de $\tau \approx 60 a$ para descorrelacionar los dos iones que forman a la mancuerna. Cuando los resultados de TPE son comparados con los resultados de IME, otra vez se encuentra un excelente acuerdo. El estudio de la fuerza media efectiva revela un acuerdo cuantitativo excelente entre TPE y DME para la fuerza entre dos iones de carga opuesta, $F_{+-}$, aunque la teoria HNC/MSA es también muy buena. Para la fuerza $\mathrm{F}_{++}$debemos remarcar la presencia de un cambio abrupto en la pendiente de la fuerza entre iones igualmente cargados cuando están separados a una distancia de $\tau=2 a$. En este caso, DME también predice este comportamiento mientras que HNC/MSA no. Por otro lado, TPE y DME muestran un acuerdo cualitativo lo que demuestra la habilidad de TPE para tomar en cuenta las correlaciones de largo alcance.

Tenemos dos conclusiones generales de este capítulo:

- En el régimen concentrado, TPE describe al MPR tan bien como las teorías previas, en este caso, como HNC/MSA.

- En el régimen diluido TPE verdaderamente mejora la descripción del MPR simétrico.

La mejor descripción (que teorías anteriores) del electrolito tipo MPR fuertemente acoplado y diluido, por TPE-HNC/MSA puede ser relevante, por ejemplo, para el estudio de las propiedades críticas $[137,138]$. Finalmente, esta teoría podría ser exitosamente aplicada al estudio de fluidos de esferas duras neutras. 


\section{Capítulo 6}

\section{Conclusiones generales y perspectivas}

En este trabajo estudiamos algunos aspectos particulares del comportamiento de fluidos cargados homogéneos e inhomogéneos. El efecto de sobrecargado es el tema principal. Como se discutió en la introducción, dicho efecto ocurre en diversos sistemas de interés tecnológico y aparece como un efecto intrínsecamente de muchos cuerpos, por lo que nos hemos basado en las teorías de líquidos para su estudio. El estudio es amplio en el sentido de que se han aplicado las técnicas y teorías mecánico estadísticas al análisis de modelos simples de diferentes sistemas de interés tecnológico. Estos modelos, si bien simples, nos proporcionan información útil para la comprensión de sistemas reales. En este trabajo hemos respondido las siguientes preguntas:

- ¿ Es real el efecto de sobrecargado o es un artificio de la teoría? R: La existencia de dicho efecto queda confirmada por el acuerdo entre la teoría, los datos de simulación molecular y el experimento.

- ¿ Cuáles son los factores que producen dicho efecto? R: En el análisis de la doble capa eléctrica de geometría cilindrica se muestra la importancia que tiene las correlaciones por tamaño en la descripción de dicho efecto. De aquí podemos resaltar el hecho de que encontramos sobrecargado en sistemas formados por iones monovalentes y diámetro mayor. En el análisis de la doble capa eléctrica de geometría plana se encuentra que ésta puede ser lescrita en términos de tres parámetros adimensionales, estos parámetros son una radida de: (i) la interacción ion-ion, (ii) la interacción ion-superficie y (iii) el volumen exciuido por los iones. Estos parámetros proveen de criterios universales parś la aparición del efecto y a través de ellos se puede establecer lo siguiente: El ejeclo te sobrecargado resulta de una combinación entre los efectos de volumen exciuito y de correlación electrostática, es decir de un balance favorable entre entropia y ererzia.

- ¿Cuáles son las consecuencias de dicho efecto? R:

1. El hecho de considerar los efictos de correlaciones de corto alcance se refleja en la forma de los perfiles de concentración, los cuales muestran oscilaciones. Las 
oscilaciones en los perfiles de concentración son una manifestación del efecto de sobrecargado. Otra manifestación del efecto de sobrecargado se presenta en la curvatura del potencial $\zeta$ como función de la densidad superficial de carga. En este trabajo se establece la concavidad de dicha función, hecho que ha sido ampliamente discutido en a literatura y que se resuelve en este estudio. La concavidad del potencial $\zeta$ es otra manifestación de las correlaciones de corto alcance y por lo tanto del efecto de sobrecargado y tiene consecuencias en los experimentos de electroforesis. En dichos experimentos, debido a este efecto, se puede producir un revertimiento en la movilidad electroforética.

2. Los efectos entrópicos tienen implicaciones en los fenómenos de adsorción. En este trabajo hemos analizado mecanismos de adsorción en los que dichos efectos juegan un papel determinante. Estos efectos son: (i) Adsorción de macroiones sobre una superficie igualmente cargada. (ii) Formación de estructuras en una superficie con carga y sin carga. (iii) Atracción efectiva entre macroiones igualmente cargados mediada por la presencia de una campo externo. Todos estos resultados son novedosos y aparentemente contraintuitivos, además de que son de importancia tecnológica en terapia génica, en la formación de capas estructuradas (layering), en el autoensamblado de estructuras en superficies, en corrosión y adsorción de asfaltenos, etc.

3. El hecho de poder describir a los fluidos cargados en términos de parámetros escalables adimensionales da coherencia a las mediciones experimentales donde se encuentran fuerzas efectivas atractivas de largo alcance. En estos experimentos, el término largo alcance significa del orden del diámetro de las partículas coloidales, las cuales están en el rango de micrómetros. De acuerdo con nuestra descripción del capítulo 3, al escalar los parámetros del sistema de manera proporcional, el comportamiento del sistema (el rango de las interacciones efectivas) escala de la misma forma. Por lo tanto, el largo alcance de las interacciones en suspensiones coloidales es el resultado de la combinación de los efectos de muchos cuerpos y el alcance de dichas interacciones es el resultado del escalamiento de los perámetros del sistema.

El tratamiento de fluidos homogén os cargados a través del formalismo de extensión a tres puntos nos arroja las siguientes :orclusiones:

- El formalismo en verdad funciona cono un método para incorporar más correlaciones en una teoría dada de ecuacionses irteyrales.

- El formalismo describe al MPR tan bien como las teorías estándar en el régimen de alta concentración y mejor a no:ahlemente cualquier descripción en el régimen diluido.

- En este trabajo se hace por p imera yez un estudio del MPR a nivel de la función de distribución de tres partículas. Dicio estudio provee más información de la física de dichos sistemas. 
- La mejor descripción del MPR a través de este formalismo abre la posibilidad de estudiar al sistema en donde las correlaciones de largo alcance desempeñan un papel determinante, p. ej. en la región cercana al punto crítico.

Nuestros resultados han respondido a las preguntas que hemos apuntado anteriormente, sin embargo, surgen nuevas interrogantes que creemos que podemos responder en el futuro.

- El desarrollo de las técnicas numéricas de solución empleadas en el formalismo cie extensión a tres puntos puede emplearse para el estudio de las interacciones entre partículas coloidales esféricas. El estudio de estas interacciones tiene también un amplio interés tecnológico y es importante tanto para la interacción electrostática como para la interacción por volumen excluido. El efecto de la forma de las partículas coloidales también puede resultar interesante ya que puede tener un interés más amplio estudiar las interacciones entre partículas en forma de varilla cilíndrica. Las técnicas de solución numérica aquí presentadas pueden extenderse de manera directa a esta geometría.

- Las técnicas aquí desarrolladas también pueden ayudar a estudiar, por ejemplo, el papel que desempeñan la correlaciones laterales en el efecto de sobrecargado así como en los efectos de adsorción. Además de que la aplicación del formalismo de extensión a tres puntos al estudio de fluidos homogéneos puede resultar exitoso para diferentes modelos de interacciones.

- Los efectos entrópicos tienen consecuencias en los estudios de capacidad diferencial en los sistemas electroquímicos, en donde este hecho no ha sido considerado. 


\section{Apéndice A}

\section{Diagramas de Mayer}

En este apéndice describiremos brevemente la construcción de los diagramas de Mayer así como algunas de sus propiedades. En la Ec. (1.17) podemos ver que un desarrollo en potencias de $\rho$ de la función $g(r)$ involucra integrales multidimensionales sobre las coordenadas de las partículas. Tales integrales son representadas en forma gráfica.

Consideremos, por ejemplo, alguna de las integrales que aparecen en la Ec. (1.17). En general, una de las integrales correspondientes a la $k$-ésima potencia del desarrollo puede expresarse como

$$
\int \ldots \int \prod_{\{i j\}} f_{i j} \mathrm{~d} \mathbf{r}_{3} \ldots \mathrm{d} \mathbf{r}_{k}
$$

donde $f_{i j} \equiv f\left(\mathbf{r}_{i j}\right)=e^{-\beta u\left(\mathbf{r}_{i j}\right)}-1$ es la función de Mayer e $\{i j\}$ representa un subconjunto de funciones tales que $i, j \leq k^{1}$. A cada integral de la forma (A.1) corresponde un diagrama. La forma de asociarle un diagrama la damos en los siguientes puntos:

1. Dicho diagrama consiste de cierto número de puntos, de los cuales, ciertos pares se encuentran conectados por ligas.

2. Los puntos representan las coordenadas de las partículas y llevan la etiqueta correspondiente.

3. Los puntos son de dos tipos: puntos blancos (o puntos de raiz) que corresponden a las coordenadas no integradas; y puntos negros (o puntos de campo), que representan las coordenadas de integración.

4. Un punto etiquetado como $i$ se asocia a las coordenadas de una partícula.

5. Una liga entre los puntos $i$ y $j$ la asociamos con una función $\eta\left(\mathbf{r}_{i j}\right)$.

6. Un diagrama simple es aquel en el que un par de puntos están conectados únicamente por una liga.

\footnotetext{
${ }^{1}$ Por el momento discutiremos las características de estos subconjuntos.
} 
7. El valor de un diagrama es el valor de la integral que representa el diagrama; el cual es una función de las coordenadas asociadas cen los puntos blancos y una funcional de las funciones asociadas con los puntos negros.

A consinuación damos un ejemplo de tres integrales con su respectiva representación:

$$
I_{1}=\iint f\left(\mathbf{r}_{13}\right) f\left(\mathbf{r}_{34}\right) f\left(\mathbf{r}_{42}\right) \mathrm{d} \mathbf{r}_{3} \mathrm{~d} \mathbf{r}_{4}=
$$

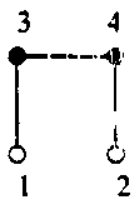

$$
I_{2}=\iint f\left(\mathbf{r}_{13}\right) f\left(\mathbf{r}_{34}\right) f\left(\mathbf{r}_{42}\right) f\left(\mathbf{r}_{14}\right) f\left(\dot{\mathbf{r}}_{32}\right) \mathrm{d} \mathbf{r}_{3} \mathrm{~d} \mathbf{r}_{4}=
$$

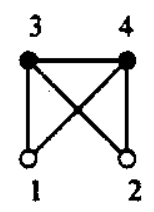

$$
I_{3}=\iiint f\left(\mathbf{r}_{13}\right) f\left(\mathbf{r}_{35}\right) f\left(\mathbf{r}_{45}\right) f\left(\mathbf{r}_{42} f\left(\mathbf{r}_{15}\right) f\left(\mathbf{r}_{25}\right) f\left(\mathbf{r}_{14}\right) \mathrm{d} \mathbf{r}_{3} \mathrm{~d} \mathbf{r}_{4} \mathrm{~d} \mathbf{r}_{5}=\right.
$$

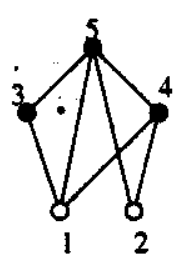

(A.2)

Los puntos negros corresponden a variables mudas. De esta forma, la manera como se encuentran etiquetados es irrelevante. El valor de un diagrama sin etiqueta involucra un factor combinatorio relacionado con la estructura topológica del diagrama. El valor de un diagrama es el mismo bajo cualquier permutación entre los índices de los puntos negros. Hay un subgrupo de diagramas que son topológicamente equivalentes. Se dice que dos diagramas son topológicamente equivalentes si están caracterizados por el mismo conjunto de conexiones. En términos de la integral a la cual representa un diagrama, equivalencia topológica significa que el integrando no se altera bajo permutación de índices. De esta forma. por definición el valor de un diagrama simple $\Gamma$ que contiene $n$ puntos blancos, etiquetados del 1 a $n$ y $m$ puntos negros, sin etiquetar es

$$
\Gamma=(1 / m !)\left\{\begin{array}{l}
\text { la suma de todos los diagramas topológicamente } \\
\text { no equivalentes obtenidos al asignar las } \\
\text { etiquetas } n+1, \ldots, n-m \text { a los puntos negros }
\end{array}\right\}
$$

Por otra parte, dos diagramas sin etiquetar son topológicamente distintos si es imposible encontrar una permutación que convierra una versión etiquetada de uno de ellos en una versión etiquetada del otro. Los diagramas topológicamente distintos representan 


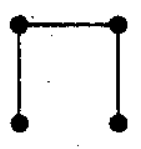

(a)

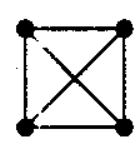

(b)

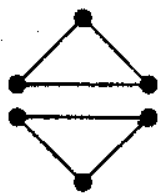

(c)

Figura A.1:

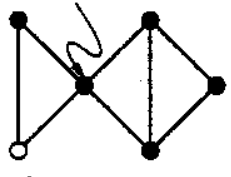

(a)

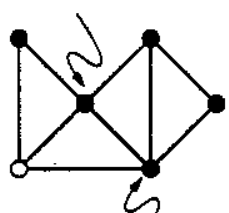

(b)

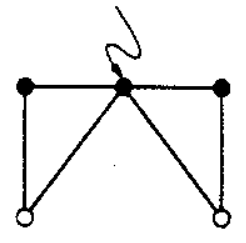

(c)

Figura A.2:

integrales distintas, aunque ocasionalmente pueden tener el mismo valor. Este tipo de diagramas son importantes debido a que las funciones mecánico estadísticas usualmente se obtienen como la suma de diagramas topológicamente distintos con ciertas propiedades.

Dos puntos son adyacentes si están conectados directamente por una liga. Una secuencia de puntos adyacentes se denomina trayectoria. Dos trayectorias entre un par dado de puntos son independientes si no tienen un punto intermedio en común. Un diagrama es disconexo si consiste de dos o más componentes; si dos puntos caen en componentes diferentes no hay trayectoria alguna entre ellos. Un diagrama conexo puede ser conexo múltiple o conexo simple. Si hay al menos $n$ trayectorias independientes entre cualquier par de puntos, se dice que el diagrama es conexo n-tuple. En la Fig. A.1 mostramos (a) un diagrama conexo simplemente (b) conexo triple y (c) un diagrama de dos componentes, las cuales son conexas doble.

Cuando se remueve un punto de un diagrama y ésto causa que el diagrama se vuelva disconexo, el punto se llama punto de conexión. Al remover una articulación de un diagrama conexo, el diagrama se descompone en dos o más componentes de las cuales al menos una no contiene puntos blancos; un par articulado es un par de puntos los cuales al ser removidos tienen el mismo efecto. Un diagrama que está libre de articulaciones se denomina irreducible. Si un diagrama contiene al menos dos puntos blancos, un punto nodal es aquel por el cual pasan todas las trayectorias entre un par particular de puntos. En la Fig. A.2 se muestran ejemplos donde señalamos con flechas (a) una articulación, (b) un par articulado (c) un punto nodal.

Finalmente, hay dos clases especiales de diagramas que son importantes en la teoría de líquidos. Éstos son las cadenas y los anillos, de los cuales mostramos un ejemplo en la Fig. A.3. 


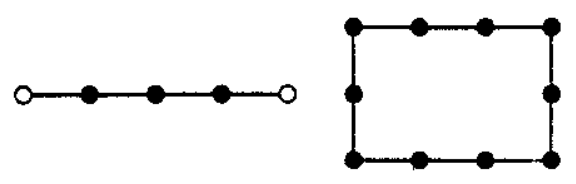

Figura A.3:

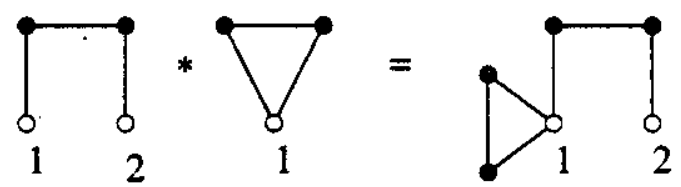

Figura A.4:

La definición de los productos estrella entre un par de diagramas es de utilidad. Sean $\Gamma_{1}$ un diagrama conexo con $n_{1}$ puntos blancos y $\Gamma_{2}$ un diagrama conexo con $n_{2}$. El producto estrella $\Gamma_{3}$ es formado uniendo $\Gamma_{1}$ y $\Gamma_{2}$ de tal forma que todos los puntos que llevan la misma etiqueta coinciden. La formacíon de un diagrama estrella es ilustrada en la Fig. A.4. Si los puntos blancos de $\Gamma_{1}$ y $\Gamma_{2}$ no tienen etiqueta alguna en común, o si algún diagrama contiene únicamente puntos negros, el producto estrella es un diagrama disconexo cuyos componentes son $\Gamma_{1}$ y $\Gamma_{2}$. Los diagramas estrella-irreducibles son diagramas conexos que no pueden ser expresados como el producto estrella de otros dos diagramas excepto cuando uno de los diagramas consiste de un solo punto blanco.

Dado que la Ec. (1.17) puede escribirse como

$$
g\left(r_{12}\right)=e^{-\beta u\left(r_{12}\right)}\left\{1+\sum_{n=1}^{\infty} \rho^{n} g_{n}\left(r_{12}\right)\right\}
$$

el $k$-ésimo coeficiente del desarrollo en serie puede escribirse como

$$
g_{k}\left(r_{12}\right)=\left\{\begin{array}{l}
\text { la suma de todos los diagramas simples que consisten de dos } \\
\text { puntos blancos etiquetados con } 1 \text { y } 2, k \text { puntos negros } \\
\text { unidos por ligas } f ; \text { sin incluir los diagramas que tienen } \\
\text { puntos de conexión }
\end{array}\right\}
$$

Una clase de diagramas denominado diagramas serie están definidos por

$$
b_{k}\left(r_{12}\right)=\left\{\begin{array}{l}
\text { todos los diagramas simples que consisten de dos } \\
\text { puntos blancos etiquetados con } 1 \text { y } 2 . k \text { puntos negros } \\
\text { unicos por ligas } f ; \text { sin incluir los diagramas que tienen } \\
\text { puntos de articulación pero que contienen al menos } \\
\text { un punto nodal }
\end{array}\right\}
$$




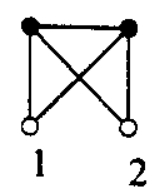

Figura A.5:

La importancia de esta clase de diagramas radica en $i$ hecho de que puede escribirse

$$
b\left(r_{12}\right) \equiv \sum_{i=1}^{\infty} \rho_{n} b_{n}\left(r_{12}\right)=h\left(r_{12}\right)-c\left(r_{12}\right)
$$

Otra clase importante de diag sonas don denominados diagramas puente tales, los cuales son

$$
d_{k}\left(r_{12}\right)=\left\{\begin{array}{l}
\text { todos los diagramas simples que consisten de dos } \\
\text { puntos blancos etiquetados con } 1 \text { y } 2, k \text { puntos negros } \\
\text { unidos por ligas } f ; \text { en donde los puntos blancos no } \\
\text { son un par articulado, no contienen puntos nodales y } \\
\text { no hay liga } f_{12}
\end{array}\right\}
$$

El único diagrama puente de segundo orden es mostrado en la Fig. A.5

Eșta clasificación de los diagramas da como resultado que el potencial de la fuerza promedio $w\left(r_{12}\right)$ se escriba como

$$
\beta w\left(r_{12}\right)=\beta u\left(r_{12}\right)-b\left(r_{12}\right)-d\left(r_{12}\right)
$$

Por lo que combinando las Ecs. (A.7) y (A.9) haciendo uso de $-\beta w\left(r_{12}\right)=\log g\left(r_{12}\right)=$ $\log \left[h\left(r_{12}\right)+1\right]$ tenemos

$$
h\left(r_{12}\right)-c\left(r_{12}\right)-\log \left[h\left(r_{12}\right)+1\right]=\beta u\left(r_{12}\right)-d\left(r_{12}\right)
$$

Dado que $h\left(r_{12}\right)$ y $c\left(r_{12}\right)$ están relacionados por medio de la ecuación de Ornstein-Zernike, la Ec. (A.10) puede escribirse como una ecuación para $h$ (o para $c$ ), en caso de que se pudiera escribir una relación para la función $d\left(r_{12}\right)$ en términos de $h(\mathrm{y} / \mathrm{o} c)$ únicamente. El desarrollo en serie de $d\left(r_{12}\right)$ puede reemplazè rse por un desarrollo en términos de $h\left(r_{12}\right)$, el cual se sustituye en la Ec. (A.10). E! resiltado, junto con la ecuación de Ornstein-Zernike, es una ecuación integral exac:a para $h\left(r_{12}\right)$, sin embargo. el desarrollo se vuelve intratable. Si hacemos la aproximacicn de $d\left(r_{12}\right)=0$ la Ec. (A.10) obtenemos una ecuación integral aproximada para $h\left(r_{12}\right)$ conocida como la aproximación de cadena hipertejida (HNC hypernetted-chain). 


\section{Apéndice B}

\section{Expresiones analíticas para los kérneles}

En la aproximación MSA,

$$
s c_{s}(s)=-\gamma_{1} s-\frac{6 \eta}{a} \gamma_{2} s^{2}-\frac{\eta !}{2 a^{3}} \gamma_{1} s^{4}
$$

y

$$
s c_{s}(s)=\frac{e^{2} \beta}{\varepsilon}\left\{1-\frac{2 \Gamma s}{(1+\Gamma a)}+\frac{\Gamma^{2} s^{2}}{(1+\Gamma a)^{2}}\right\}
$$

para $s \leq a$ y cero en otro caso. Las constantes están dadas por

$$
\begin{gathered}
\Gamma a=-\frac{1}{2}+\frac{1}{2} \sqrt{1+2 \kappa a}, \\
\kappa^{2}=\frac{4 \pi \beta e^{2}}{\varepsilon} \sum_{i=1}^{n} \rho_{i} z_{i}^{2} \\
\eta=\frac{1}{6} \pi a^{3} \sum_{i=1}^{n} \rho_{i} \\
\gamma_{1}=\frac{(1+2 \eta)^{2}}{(1-\eta)^{4}} \\
\gamma_{2}=-\frac{\left(1+\frac{1}{2} \eta\right)^{2}}{(1-\eta)^{4}} \\
\gamma_{3}=\frac{\Gamma}{(1+\Gamma a)}
\end{gathered}
$$

De aquí que las expresiones para los kérneles estás dadas por

$$
K(x, y)=\frac{\gamma_{1}}{2}\left[(x-y)^{2}-a^{2}\right]+\frac{2 \eta}{a} \gamma_{2}\left[|x-y|^{3}-a^{3}\right]+\frac{\eta \gamma_{1}}{10 a^{3}}\left[|x-y|^{5}-a^{5}\right]
$$


para $x-a \leq y \leq x+a, y$

$L(x, y)=\frac{e^{2} \beta}{\varepsilon}\left\{[a-|x-y|]-\frac{\Gamma}{(1+\Gamma a)}\left[a^{2}-(x-y)^{2}\right]+\frac{1}{3}\left(\frac{\Gamma}{(1+\Gamma a)}\right)^{2}\left[a^{3}-(x-y)^{3}\right]\right\}$

para $x-a \leq y \leq x+a$. La expresión para $J(x)$ se puede obtener analíticamente

$$
J(x)=2 \pi \rho_{T}\left\{\frac{1}{6} \gamma_{1}\left[p^{3}-3 a^{2} p+2 a^{3}\right]+\frac{\eta \gamma_{2}}{2 a}\left[p^{4}-4 a^{3} p+3 a^{4}\right]+\frac{\eta \gamma_{1}}{60 a^{3}}\left[p^{6}-6 a^{5} p+5 a^{6}\right]\right\},
$$

para $a / 2 \leq x \leq \frac{3}{2} a$ y cero de cualquier otra forma. Hemos cefinido a $p \equiv x-a / 2$. 


\section{Apéndice $\mathrm{C}$}

\section{La aproximación esférica media}

Las expresiones para la función de correlación directa, de un electrolito tipo modelo primitivo han sido obtenidas por Blum y Hiroike, a través de la solución de la ecuación de Ornstein-Zernike, en el bulto $[139,140,141]$. Las expresiones de la función de correlación directa pueden escribirse como

$$
c_{i j}(s)=c_{i j}^{S R}(s)+c_{i j}^{H S}(s)-\beta \frac{z_{i} z_{j} e^{2}}{\varepsilon s},
$$

donde

$$
c_{i j}^{S R}(s)=\left\{\begin{array}{lll}
\frac{e^{2} \beta}{\varepsilon}\left(\zeta_{i j}+\frac{z_{i} z_{j}}{s}\right), & \text { para } 0 \leq s \leq \lambda_{i j} \\
\frac{e^{\frac{2}{\beta}}}{\varepsilon}\left(\frac{\alpha_{i j}+z_{i} z_{j}}{s}-\gamma_{i j}+\eta_{i j} s+\varsigma_{i j} s^{3}\right) & \text { para } \lambda_{i j}<s \leq a_{i j} \\
0, & \text { para } s>a_{i j}
\end{array}\right.
$$

con $\lambda_{i j} \equiv \frac{\left|a_{i}-a_{j}\right|}{2}$ y $a_{i j} \equiv \frac{a_{i}+a_{j}}{2}$. La función $c_{i j}^{H S}(s)$ es la función de correlación directa para una mezcla binaria de esferas duras en la aproximación de PY. Para las especies de partículas del mismo tamaño está dada por

$$
c_{i i}^{H S}(s)=\left\{\begin{array}{lll}
-\nu_{i}-\chi_{i} s-\delta s^{3}, & \text { si } s<a_{i} \\
0, & \text { si } s>a_{i}
\end{array}\right.
$$

Para partículas de diferente tamaño está dada por

$$
c_{13}^{H S}(s)=\left\{\begin{array}{lll}
\alpha_{1}, & \text { si } s \leq \lambda, \\
\alpha_{1}+\frac{\left[v x^{2}+4 \lambda \delta x^{3}+\delta x^{4}\right]}{s} & \text { si } \lambda<s \leq a_{13} \\
0 & \text { si } & s>a_{13}
\end{array}\right.
$$

con $x=s-\lambda_{13}$ y las constantes dadas por 


$$
\begin{gathered}
s_{i}=\left(n_{i}+\Gamma x_{i}\right) \\
\zeta_{i j}=2\left[z_{i} n_{j}-x_{i} s_{i}+\frac{a_{i}}{3} s_{i}^{2}\right] \\
\alpha_{i j}=\left(a_{i}-a_{j}\right)\left\{\frac{\left(x_{i}+x_{j}\right)}{4}\left[s_{i}-s_{j}\right]-\frac{\left(a_{i}-a_{j}\right)}{16}\left[\left(n_{i}+\Gamma x_{i}+n_{j}+\Gamma x_{j}\right)^{2}-4 n_{i} n_{j}\right]\right\} \\
\gamma_{i j}=\left(x_{i}-x_{j}\right)\left(n_{i}-n_{j}\right)+\left(x_{i}^{2}+x_{j}^{2}\right) \Gamma+\left(a_{i}+a_{j}\right) n_{i} n_{j}-\frac{1}{3}\left[a_{i} s_{i}^{2}+a_{j} s_{j}^{2}\right] \\
\eta_{i j}=\frac{x_{i}}{a_{i}} s_{i}+\frac{x_{j}}{a_{j}} s_{j}+n_{i} n_{j}-\frac{1}{2}\left[s_{i}^{2}+s_{j}^{2}\right] \\
s_{i j}=\frac{s_{j}}{6 a_{j}^{2}}+\frac{s_{i}}{6 a_{i}^{2}}
\end{gathered}
$$

donde $\Gamma$ se obtiene de la solución de la siguiente ecuación algebráica

$$
\Gamma^{2}=\frac{\pi e^{2} \beta}{\varepsilon} \sum_{i=1}^{n} \rho_{i}\left(z_{i}+n_{i} a_{i}\right)^{2} .
$$

Las constantes $n_{i}$ se obtienen de la solución del siguiente sistema algebráico

$$
-\left(z_{i}+n_{i} a_{i}\right) \Gamma=n_{i}+c a_{i} \sum_{j=1}^{n}\left(z_{i}+n_{j} a_{i}\right)
$$

donde $c=\frac{\pi}{2}\left[1-\frac{\pi}{6} \sum_{j=1}^{n} \rho_{i} a_{i}^{3}\right]^{-1}, \mathrm{y}$ las $x_{i}$ están definidas como $x_{i} \equiv z_{i}+n_{i} a_{i}$.

$$
\begin{gathered}
\nu_{1}=\nu_{2}=\frac{\partial \beta p\left(\rho_{1}+\rho_{2}, \rho_{3}\right)}{\partial\left(\rho_{1}+\rho_{2}\right)} \\
\nu_{3}=\frac{\partial \beta p\left(\rho_{1}+\rho_{2}, \rho_{3}\right)}{\partial \rho_{3}}, \\
\nu_{3}=\frac{\partial \beta p\left(\rho_{1}+\rho_{2}, \rho_{3}\right)}{\partial \rho_{3}}, \\
\chi_{1}=-6\left[\eta_{1} a^{2} g_{11}^{2}(a)+\eta_{3} a_{3} g_{33}\left(a_{3}\right)\right] \\
v=-6\left[\eta_{1} a g_{11}(a)+\eta_{3} a_{3} g_{33}\left(a_{3}\right)\right] a_{13} g_{13}\left(a_{13}\right),
\end{gathered}
$$




$$
\begin{gathered}
\delta=\frac{1}{2}\left[\eta_{1} \nu_{1}+\eta_{3} \nu_{3}\right], \\
\beta p=\frac{\left(\rho_{1}+\rho_{2}+\rho_{3}\right)\left[1+\xi+\xi^{2}\right]}{\left(1-\xi^{3}\right)} \\
-\frac{18 \eta_{1} \eta_{3}\left(a_{3}-a\right)\left[\left(a+a_{3}\right)+a a_{3}\left(\eta_{1} a_{1}^{2}+\eta_{3} a_{3}^{2}\right)\right]}{\pi\left(1-\xi^{3}\right)} \\
g_{11}(a)=\left\{\left[1+\frac{1}{2} \xi\right]+\frac{3}{2} \eta_{3} a_{3}^{3}\left(a-a_{3}\right)\right\}(1-\xi)^{-2}, \\
g_{13}\left(a_{13}\right)=\left[a_{3} g_{11}(a)+a g_{33}\left(a_{3}\right)\right], \\
\xi=\eta_{1} a^{3}+\eta_{3} a_{3}^{3}, \\
\eta_{1}=\frac{\pi}{6}\left(\rho_{1}+\rho_{2}\right), \\
\eta_{3}=\frac{\pi}{6} \rho_{3},
\end{gathered}
$$

$\chi_{3}, g_{33}\left(a_{3}\right)$ se obtienen al intercambiar $\eta_{1}, a_{1}$ con $\eta_{3}, a_{3}$, en las expresiones para $\chi_{1}, g_{11}(a)$.

C.1 Las expresiones para los kérneles

$$
\begin{gathered}
K_{j i}(x, y)=\int_{|x-y|}^{\infty} c_{j i}^{H S}(s) s d s, \\
K_{i i}(x, y)=-\frac{\nu_{i}}{2}\left(a_{i i}^{2}-(x-y)^{2}\right)-\frac{\chi_{i}}{3}\left(a_{i i}^{3}-|x-y|^{3}\right)-\frac{\delta}{5}\left(a_{i i}^{5}-|x-y|^{5}\right),
\end{gathered}
$$

para $a_{i i} \geq|x-y|$.

$$
\begin{gathered}
K_{i i}(x, y)=0 \text { para } a_{i i} \leq|x-y| \\
K_{13}(x, y)=K_{31}(x, y)=\frac{\alpha_{1}}{2}\left(a_{13}^{2}-(x-y)^{2}\right)+\frac{v}{3} a^{3}+\delta \lambda a^{4}+\frac{\delta}{5} a^{5}, \\
\text { para }|x-y| \leq \lambda
\end{gathered}
$$

$$
\begin{aligned}
& K_{13}(x, y)=K_{31}(x, y)=\frac{\alpha_{1}}{2}\left(a_{13}^{2}-(x-y)^{2}\right)+\frac{v}{3}\left(a^{3}-(|x-y|-\lambda)^{3}\right)+ \\
& \delta \lambda\left(a^{4}-(|x-y|-\lambda)^{4}\right)+\frac{\delta}{5}\left(a^{5}-(|x-y|-\lambda)^{5}\right),
\end{aligned}
$$


para $\lambda<|x-y| \leq a_{13}$.

$$
\begin{gathered}
\left.K_{13}(x, y)=0, \text { si } a_{13}<\mid x-\right\} \mid \\
L_{j i}(x, y)=\int_{|x-y|}^{\infty} c_{j i}^{S R}(s) s d s \\
L_{i j}(s)=\frac{e^{2} \beta}{\varepsilon}\left\{\frac{\zeta_{i j}}{2}\left(\lambda_{i j}^{2}-(x-y)^{2}\right)+z_{i} z_{j}\left(a_{i j}-|x-y|\right)+\alpha_{i j}\left(a_{i j}-\lambda_{i j}\right)-\right. \\
\left.\left.\frac{\gamma_{i j}}{2}\left(a_{i j}^{2}-\lambda_{i j}^{2}\right)+\frac{\eta_{i j}}{3}\left(a_{i j}^{3}-\lambda_{i j}^{3}\right)+\bigcap_{i j}^{5}-\lambda_{i j}^{5}\right)\right\}
\end{gathered}
$$

para $0 \leq|x-y| \leq \lambda_{i j}$

$$
\begin{aligned}
& L_{i j}(x, y)=\frac{e^{2} \beta}{\varepsilon}\left\{\left(\alpha_{i j}+z_{i} z_{j}\right)\left(a_{i j}-|x-y|\right)-\frac{\gamma_{i j}}{2}\left(a_{i j}^{2}-|x-y|^{2}\right)\right. \\
& \left.+\frac{\eta_{i j}}{3}\left(a_{i j}^{3}-|x-y|^{3}\right)+\frac{\varsigma_{i j}}{5}\left(a_{i j}^{5}-|x-y|^{5}\right)\right\}
\end{aligned}
$$

para $\lambda_{i j}<|x-y| \leq a_{i j}$

$$
L_{i j}(x, y)=0, \quad \text { si } \quad a_{i j}<|x-y|
$$




\section{Apéndice D}

\section{El método numérico}

\section{D.1 El método de elemento finito}

Para resolver la ecuación TPE-HNC/MSA, Ec. (5.18), se tienen que usar técnicas de métodos numéricos debido a la imposibilidad para obtener soluciones analíticas. El método de elemento finito (MEF) ya ha sido usado anteriormente para resolver la ecuación HNC/MSA en varias geometríac $[16,102]$ y ha demostrado ser un método eficiente. En este apéndice describimos el MEF usado para resolver la ecuación (5.18). Partiendo de la forma general de la ecuación integral TPE-HNC/MSA, la cual viene dada por la forma

$$
g_{\alpha i}(\eta, \xi)=\exp \left\{\mathrm{M}_{\mathrm{i}}(\eta, \xi)+\int_{-1}^{1} \int_{\eta_{0}(\xi)}^{\infty} \sum_{\mathrm{m}=1}^{2} \rho_{\mathrm{m}} \mathrm{h}_{\alpha \mathrm{m}}\left(\eta^{\prime}, \xi^{\prime}\right) \mathrm{F}\left(\eta, \xi, \eta^{\prime}, \xi^{\prime}\right) \mathrm{d} \eta^{\prime} \mathrm{d} \xi^{\prime}\right\}
$$

donde $h_{\alpha m}(\eta, \xi)$ es una función definida en el dominio bidimensional $(\eta, \xi) \in[-1,1] \times$ $[1, \infty)$. Dado que, $h_{\alpha m}(\eta, \xi) \neq 0$ únicamente en una región cercana a la mancuerna, podemos resolver la ecuación integral en un dominio finito. En el MEF [142] el dominio queda dividido en $N$ elementos. A su vez cada elemento, en un dominio $A_{K}$, es dividido en $L_{0}$ sub-elementos. Por conveniencia, ciebe buscarse la geometría más simple de los elementos finitos. En nuestro sistema cuordenalo. la geometría de las dos esferas es nostrado en la Fig. D.1a así como la división del espacio de solución. Con el fin de obtener geometrías simples de los elemeitus finitos, el espacio de solución en coordenadas $(x, y)$ es mapeado en un espacio d $\epsilon$ crordenadas $(\xi, \eta)$, en el cual se obtiene la geometría de la Fig. D.1b.

Para llevar a cabo la solución de la. Ec. (D.J), se hace un desarrollo de la función como una combinación lineal de $L_{\text {: }}$ eienıentos ce una base $\left\{\phi_{i}^{K}(\eta, \xi), i=1 \ldots, L_{0}\right\}$, en el $K$-ésimo elemento. Dado que hay $N$ elementos. el desarrollo se escribe como

$$
h_{\alpha m}(\eta, \xi):=\sum_{K=1}^{N} \sum_{l=1}^{L} u_{m l}^{K} \phi_{l}^{K}(\eta, \xi), \mathrm{m}=1,2
$$



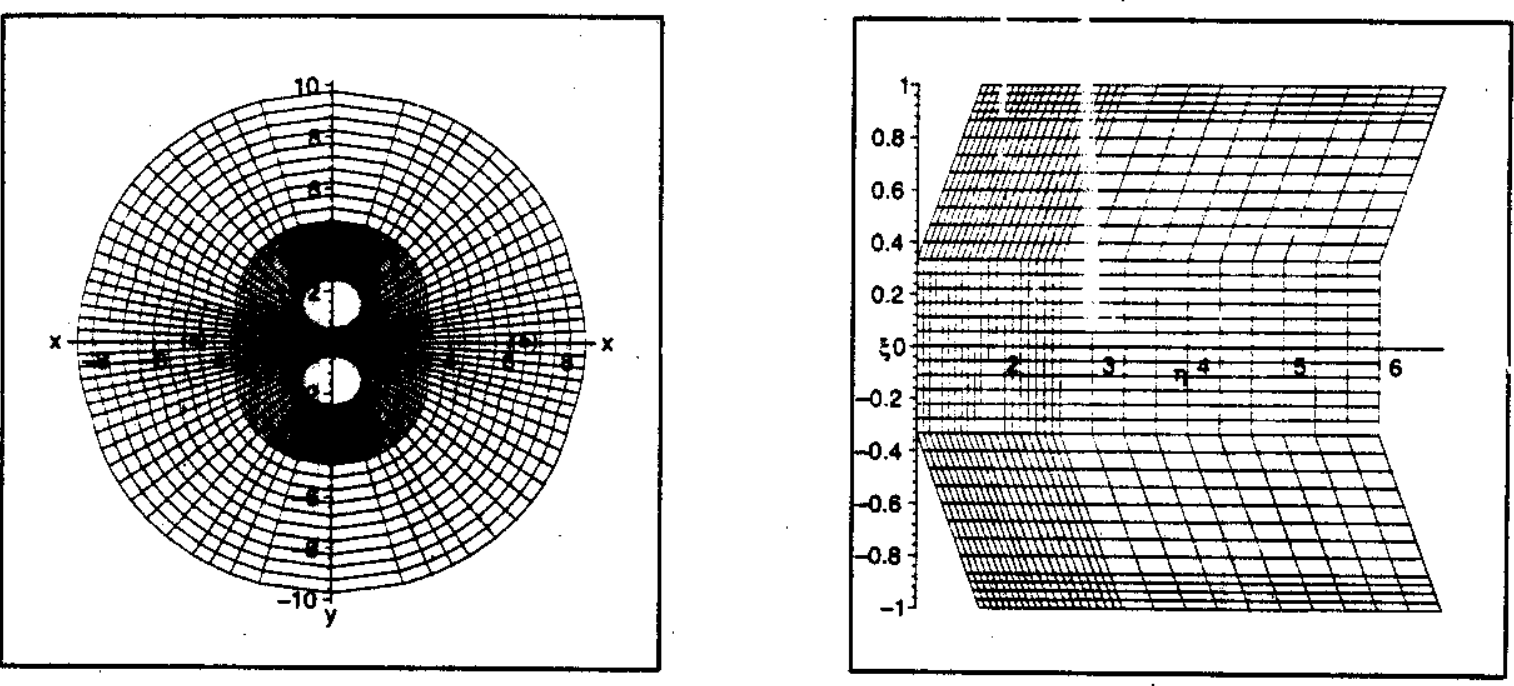

Figura D.1: (a) Un ejemplo de la malla usada en la solución de la Ec.(D.1), en coordenadas cartesianas. (b) La misma malla mostrada en (a) pero en el plano $\eta-\xi$.
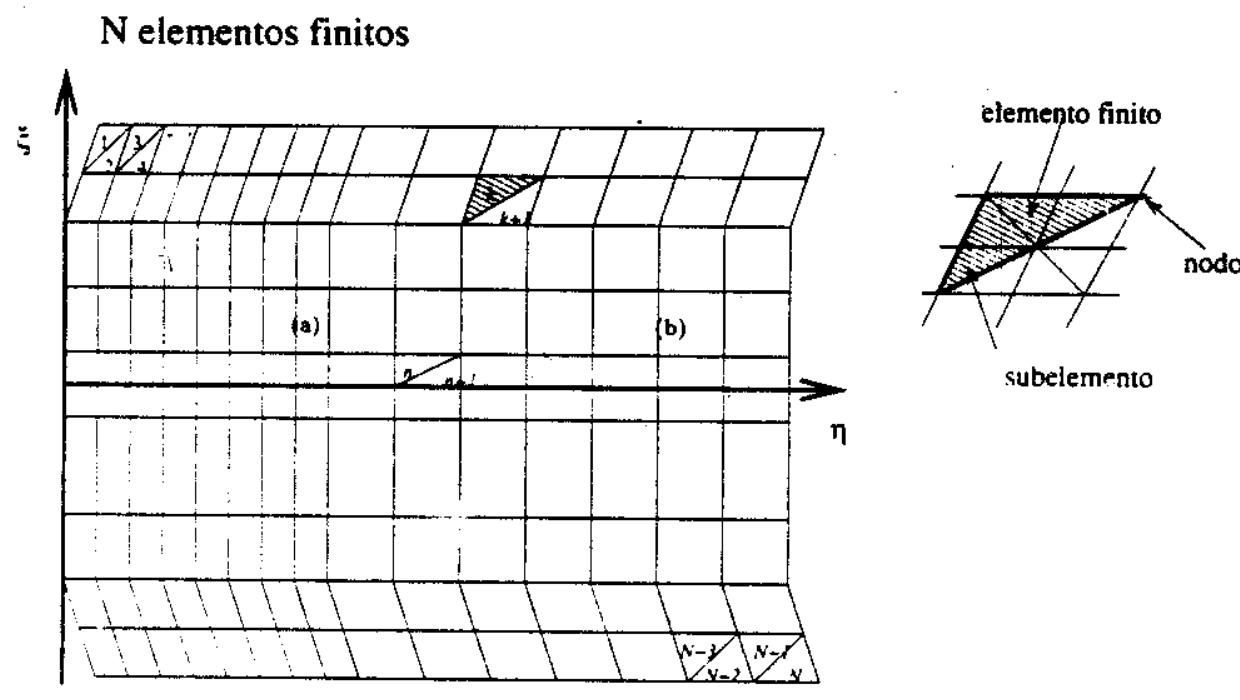

Elemento finito

Figura D.2: (a) Un ejemplo de la enumeración de los elemeritos finitos. (b) División de un elemento finito en subelementos. También mostramos la lucalización de los nodos. 
donde $\left\{\omega_{m l}^{K}, l=1, \ldots, L_{0}\right\}$ son los $L_{0}$ coeficientes de la función $h_{\alpha m}$ en el $K$-ésimo elemento finito. Los elementos base son definidos de tal forma que $\phi_{l}^{K}(\eta, \xi)=0$ si $(\eta, \xi) \notin A_{K}$. También los elementos base se eligen de ta forina que, para un conjunto de $L_{0}$ puntos $\left(\eta_{j}, \xi_{j}\right)$ (ver Fig. D.2), llamados nodos, las funciones base satisfacen

$$
\phi_{i}^{K}\left(\eta_{j}, \xi_{j}\right)=\delta_{i j}, \mathrm{i}, \mathrm{j}=1, \ldots, \mathrm{L}_{0} .
$$

siendo $\delta_{i j}$ la función delta de Kronecker. En la Fig. D.2 los nodos son todos los puntos de intersección en el elemento $K$-ésimo. Por lo tanto, el coeficiente $\omega_{m i}^{K}$, es el valor de la función $h_{\alpha m}$ en el $i$-ésimo nodo, i. e.,

$$
\omega_{m i}^{K}=h_{\alpha m}\left(\eta_{i}, \xi_{i}\right)
$$

Podemos reenumerar los elemientos base y a los coeficientes también, de esta forma la Ec. (D.2) es reescrita como

$$
h_{\alpha m}(\eta, \xi)=\sum_{l=1}^{M_{0}} \omega_{m l} \phi_{l}(\eta, \xi), \mathrm{m}=1,2
$$

y

$$
\omega_{m i}=h_{\alpha m}\left(\eta_{i}, \xi_{i}\right)=g_{\alpha m}\left(\eta_{i}, \xi_{i}\right)-1
$$

Con $M_{0}=N \times L_{0}, y$ el índice superior es omitido. Sustituyendo la ecuación (D.5) en la ecuación (D.1), obtenemos

$$
g_{\alpha i}(\eta, \xi)=\exp \left\{\mathrm{M}_{\mathrm{i}}(\eta, \xi)+\sum_{\mathrm{l}=1}^{\mathrm{M}_{0}} \sum_{\mathrm{m}=1}^{2} \rho_{\mathrm{m}} \omega_{\mathrm{ml}} \mathrm{C}_{\mathrm{l}}(\eta, \xi)\right\}
$$

donde hemos definido

$$
C_{l}(\eta, \xi)=\iint_{A_{K}} \phi_{l}\left(\eta^{\prime}, \xi^{\prime}\right) F\left(\eta^{\prime}, \xi^{\prime}, \eta, \xi\right) d \eta^{\prime} d \xi^{\prime}
$$

Evaluando la ecuación (D.7) en el $k$-ésimo nodo y usando la ecuación (D.6), obtenemos

$$
\omega_{i k}=\exp \left\{\mathrm{M}_{\mathrm{ik}}+\sum_{\mathrm{l}=1}^{\mathrm{M}_{0}} \sum_{\mathrm{m}=1}^{2} \rho_{\mathrm{m}} \omega^{\prime} \mathrm{ml} \mathrm{C}_{\mathrm{k}}\right\}-1,
$$

donde $C_{l k} \equiv C_{l}\left(\eta_{k}, \xi_{k}\right)$ y $\mathrm{M}_{i k} \equiv \mathrm{M}_{i}\left(\eta_{k}, \xi_{k}\right)$. De esta forma, tenemos un sistema de $2 M_{0}$ ecuaciones algebraicas no lineales, el cual es rescelio no: cualquiera de los métodos estándar de solución, por ejemplo el método de $N$ en ton. Le integral $C_{l k}$, en el K-ésimo elemento la evaluamos como una suma de las integ ales sobre cada uno de los subelementos. De esta forma,

$$
C_{l k}=\sum_{m=1}^{4} C_{l k}^{(r i)}
$$

donde $C_{l k}^{(m)}$ es la integral sobre el $m$-ésir to subeleme atc. Este procedimiento ha sido empleado con el fin de obtener mejor precisión en la evaluación de la integral. 


\section{D.2 Las coordenadas de área}

Las funciones base, $\left\{\phi_{i}^{K}(\eta, \xi), i=1, \ldots, L_{0}\right\}$, se construyen usando un sistema de coordenadas definido sobre el K-ésimo elemento. Éstas son definidas de la siguiente forma,

$$
L_{i}=\frac{a_{i}+b_{i} \eta+c_{i} \xi}{2 \Delta}, \quad \text { para } i=1,2,3
$$

donde $A=2 \Delta$ es el área del elemento triangular y

$$
\begin{aligned}
a_{i} & =\eta_{j} \xi_{k}-\eta_{k} \xi_{j}, \\
b_{i} & =\xi_{j}-\xi_{k}, \quad \text { con } i, j, k=1,2,3 \text { pero } i \neq j \neq k \\
c_{i} & =\eta_{k}-\eta_{j},
\end{aligned}
$$

con rotación cíclica de índices. El conjunto de puntos $\left\{\left(\xi_{i}, \eta_{i}\right), i=1,2,3\right\}$ son las coordenadas de los vértices del triángulo. En la Fig. D.3 mostramos la localización de estos puntos. Por otra parte la interpretación geométrica de estas coordenadas está dada en términos de

$$
L_{i}=\frac{A_{i}}{A_{i}} \quad \operatorname{con} i=1,2,3
$$

donde $A_{i}$ son las áreas de los triángulos mostrados en la Fig. D.3.

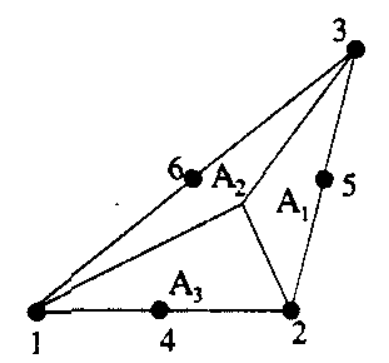

Figura D.3: Representación geométrica de las coordenadas de área y los nodos del elemento finito

La relación entre el sistema coordenado $(\xi, \eta)$ y las coordenadas triangulares está dado por

$$
\begin{aligned}
& \eta=L_{1} \eta_{1}+L_{2} \eta_{2}+L_{3} \eta_{3}, \\
& \xi=L_{1} \xi_{1}+L_{2} \xi_{2}+L_{3} \xi_{3} . \\
& 1=L_{1}+L_{2}+L_{3} .
\end{aligned}
$$

El número de nodos es igual al número de elementos base. Para la solución de la ecuación (D.1) hemos usado una base cuadrática, por lo que el desarrollo sobre el elemento finito 
es llevado a cabo con $L_{0}=6$, el cual corresponde número de funciones base, las cuales son definidas como a continuación se muestra

$$
\phi_{1}=\left(2 L_{1}-1\right) L_{1}, \text { etc. }
$$

y para los nodos que están a los lados

$$
\phi_{4}=4 L_{1} L_{2}, \text { etc. }
$$

Donde la numeración de los nodos se sigue de acuerdo con la Fig. D.3. La integral sobre el $m$-êsimo subelemento se puede escribir como

$$
C_{l}^{(m)}(\eta, \xi)=4 \Delta_{m} \int_{0}^{1} \int_{0}^{1-K_{2}} \phi_{l}\left(L_{1}, L_{2}\right) F\left(L_{1}, L_{2}, \eta, \xi\right) d K_{1} d K_{2}
$$

Donde $2 \Delta_{m}$ es el área del $m$-ésimo subelemento y $\left\{K_{j} ; i \stackrel{\doteq}{=} 1 \ldots 3\right\}$ son coordenadas de área definidas sobre el mismo elemento de forma análoga a la definición del conjunto $\left\{L_{i} ; i=\right.$ $1 \ldots 3\}$, pero éstas están definidas sobre un subelemento, desde luego que $L_{i}=L_{i}\left(K_{1}, K_{2}\right)$. La integral en la ecuación (D.15) puede ser evaluada como

$$
\int_{0}^{1} \int_{0}^{1-K_{2}} \phi_{l}\left(L_{1}, L_{2}\right) F\left(L_{1}, L_{2}, \eta, \xi\right) d K_{1} d K_{2}=\frac{1}{2} \sum_{l=1}^{n} \varrho_{l} \phi_{l}\left(K_{1}^{(l)}, K_{2}^{(l)}\right) F\left(K_{1}^{(l)}, K_{2}^{(l)}, \eta, \xi\right)
$$

Tabla D.1: Valor de los parámetros usados en la evaluación de la integral (D.16)

\begin{tabular}{ccccc}
\hline \hline$l$ & $K_{1}^{(l)}$ & $K_{2}^{(l)}$ & $K_{3}^{(l)}$ & $\varrho_{l}$ \\
\hline \hline 1 & $\frac{1}{3}$ & $\frac{1}{3}$ & $\frac{1}{3}$ & -0.1495700444 \\
2 & $\alpha_{1}$ & $\alpha_{2}$ & $\alpha_{2}$ & 0.1756152574 \\
3 & $\alpha_{2}$ & $\alpha_{2}$ & $\alpha_{1}$ & 0.1756152574 \\
4 & $\alpha_{2}$ & $\alpha_{1}$ & $\alpha_{2}$ & 0.1756152574 \\
5 & $\beta_{1}$ & $\beta_{2}$ & $\beta_{2}$ & 0.0533472356 \\
6 & $\beta_{2}$ & $\beta_{2}$ & $\beta_{1}$ & 0.0533472356 \\
7 & $\beta_{2}$ & $\beta_{1}$ & $\beta_{2}$ & 0.0533472356 \\
8 & $\gamma_{1}$ & $\gamma_{2}$ & $\gamma_{3}$ & 0.0771137608 \\
9 & $\gamma_{1}$ & $\gamma_{3}$ & $\gamma_{2}$ & 0.0771137608 \\
10 & $\gamma_{2}$ & $\gamma_{1}$ & $\gamma_{3}$ & 0.0771137608 \\
11 & $\gamma_{2}$ & $\gamma_{3}$ & $\gamma_{1}$ & 0.0771137608 \\
12 & $\gamma_{3}$ & $\gamma_{1}$ & $\gamma_{2}$ & 0.0771137608 \\
13 & $\gamma_{3}$ & $\gamma_{2}$ & $\gamma_{1}$ & 0.0771137608 \\
\hline
\end{tabular}


Con el siguiente valor para las constantes

$$
\begin{array}{ll}
\alpha_{1}=0.4793080678 & \alpha_{2}=0.2603459660 \\
\beta_{1}=0.8697397941 & \beta_{2}=0.0651301029 \\
\gamma_{1}=0.6384441885 & \gamma_{2}=0.3128654960 \\
\gamma_{3}=1-\gamma_{1}-\gamma_{2} &
\end{array}
$$

El cálculo de los coeficientes $C_{l k}$ eit la Ec. (D.9), a través de la ecuación (D.15) inás la solución de la ecuación (D.9) es un proceso que puede tomar varias horas de cálculo, para un conjunto dạdo de parámetros del sistema. 


\section{Bibliografía}

[1]. P. C. Himenz, Principles of colloid and surface chemistry, 1st ed. (Marcel Dekker, Inc., New York and Basel, 1977).

[2] W. B. Russel, D. A. Saville, y W. R. Schowalter, Colloidal Dispersions (Cambridge University Press, Cambridge, 1989).

[3] T. Yoshiyama, I. Sogami, y N. Ise, Phys. Rev. Lett. 53, 2153 (1984).

[4] H. Yoshida, N. Ise, y T. Hashimoto, J. Chem. Phys. 103, 10146 (1995).

[5] E. J. W. Verwey y J. T. G. Overbeek, Theory of the Stability of Lyophobic Colloids (Elsevier, Amsterdam, 1948).

[6] B. V. Derjaguin y L. Landau, Acta Physicochim. URSS 14, 633 (1942).

[7] B. V. Derjaguin y L. Landau, J. Exp. Theor. Phys 11, 802 (1941).

[8] B. V. Derjaguin y L. Landau, J. Exp. Theor. Phys 15, 662 (1945).

[9] G. M. Kepler y S. Fraden, Phys. Rev. Lett. 73, 356 (1994).

[10] M. D. Carbajal-Tinoco y J. L. Arauz-Lara, Phys. Rev. E 56, 6962 (1997).

[11] J. C. Crocker y D. Grier, Phys. Rev. Lett. 77, 1897 (1996).

[12] T. M. Squires y M. P. Brenner, Phys. Rev. Lett. 85, 476 (2000).

[13] O. Lambert, L. Letellier, W. M. Gelbart, y J.-L. Rigaud, en Proceedings of the National Academy of Science (National Academy of Science (USA), USA, 2000), Vol. 97 , pp. $7248-7253$;.

[14] R. Golestanian, M. Kardar, y T. B. Liverpool, Phys. Rev. Lett. 82, 4456 (1999).

[15] M. Lozada-Cassou y E. Díaz-Herrera, J. Chem. Fhys. 92, 1194 (1990).

[16] M. Lozada-Cassou y E. Díaz-Herrera, J. Chem. Phys. 93, 1386 (1990).

[17] J. E. Sánchez y M. Lozada-Cassou, Ċhem. Phys.Lett. 190, 202 (1992). 
[18] S. L. Carnie y D. Chan, J. Colloid Interface Sci. 155, 297 (1992).

[19] S. L. Carnie, D. Chan, y J. Stankovich, J. Colloid Interface Sci. 165, 116 (1993).

[20] B. Y. Ha y A. J. Liu, Phys. Rev. Lett. 79, 1289 (1997).

[21] W. R. Bowen y A. O. Sharif, Nature 393, 663 (2000).

[22] E. Allahyarov y H. Löwen, Phys. Rev. E 63, 041403 (2001).

[23] R. Messina, C. Holm, y K. Kremer, Phys. Rev. Lett. 85, 872 (2000).

[24] R. Messina, C. Holm, y K. Kremer, Europhys. Lett. 51, 461 (2000).

[25] R. Messina, C. Holm, y K. Kremer, Phys. Rev. E 64, 021405 (2001).

[26] G. Gouy, J. Phys. 9, 457 (1910).

[27] D. L. Chapman, Philos. Mag. 25, 475 (1913).

[28] L. B. Bhuiyan, C. W. Outhwaite, y S. Levine, Mol. Phys. 42, 1271 (1981).

[29] E. Gonzales-Tovar, M. Lozada-Cassou, y D. Henderson, J. Chem. Phys. 83, 361 (1985).

[30] W. van Megen y I. Snook, J. Chem. Phys. 73, 4656 (1980).

[31] G. M. Torrie y J. P. Valleau, J. Phys. Chem. 86, 3251 (1982).

[32] I. Koltover, T. Salditt, J. O. Rädler, y C. R. Safinya, Science 281, 78 (1998).

[33] J. O. Rädler, I. Koltover, T. Salditt, y C. R. Safinva, Sience 275, 810 (1997).

[34] W. M. Gelbart, R. F. Bruinsma, P. A. Pincus, y V. A. Parsegian, Pysics Today 59, $38(2000)$.

[35] M. Lozada-Cassou, E. González Tovar, y W. Olivares, Phys. Rev. E 60, R17 (1999).

[36] B. I. Shklovskii. Phys. Rer. E 60, 5802 (1999).

[37] T. T. Nguyen, A. Y. Grosberg, y B. I. Shklovskii. J. Phys. Rev. Lett. 85, 1568 $(2000)$.

[38] P. Attard, J. Phys. Chem. 99, 14174 (1995).

[39] J. Ennis, S. Marcelja, y R. Kjellander, Electrochemica Acta 41, 2115 (1996).

[40] M. Deserno, F. Jiménez-Ángeles, C. Holm, y M. Lozada-Cassou, J. Phys. Chem. B 105, $10983(2001)$. 
[41] M. Deserno, C. Holm, y S. May, Macromolecules 33, 199 (2000).

[42] M. Tanaka y A. Y. Grosberg, J. Chem. Phys. 115, 567 (2001).

[43] T. Terao y T. Nakayama, Phys. Rev. E 63, 041401 (2001).

[44] F. Artzner, R. Zantl, G. Rapp, y J. O. Rädler, Phys. Rev. Lett. 81, 5051 (1998).

[45] I. K. J. O. Rädler et al., Phys. Rev. Lett. 82, 3184 (1999).

[46] D. Henderson, M. Lozada-Cassou, y L. Blum, J. Chem. Phys. 79, 3055 (1983).

[47] S. A. Rogde y B. Hafskjold, Mol. Phys. 48, 1241 (1983).

[48] E. González-Tovar, M. Lozada-Cassou, y M. Medina-Noyola, J. Chem. Phys. 95, 6784 (1991).

[49] F. Reif, Fundamentals of statistical and thermal physics (McGraw-Hill Book Company, New York, 1965).

[50] D. L. Goodstein, States of matter (Dover Publications Inc., New York, 1985).

[51] L. García-Colín Sherer, Introducción a la termodinámica clásica (Trillas, México, 1986).

[52] H. L. Friedman, A Course in Statistical Mechanics (Prentice Hall, Englewood Cliffs, NJ, 1985).

[53] D. A. McQuarrie, Statistical Mechanics (Harper y Row, New York, 1976).

[54] J. P. Hansen y I. R. McDonald, Theory of simple liquids, 2nd ed. (Academic Press, London, 1986).

[55] T. L. Hill, Statistical Mechanics (Dover Publications Inc., New York, 1987).

[56] M. Lozada-Cassou, en Fundamentals of inhomogeneous fluids (Marcel Dekker, Inc., New York, 1992), Chap. 8.

[57] L. S. Ornstein y F. Zernike, Proc. Akad. Sci. (Amsterdam) 17, 793 (1914).

is $\quad[58]$ F. Zernike, Proc. Akad. Sci. (Amsterdam) 18, 1520 (1916).

[59] E. Waisman y J. L. Lebowitz, J. Chem. Fhys. 52, 4307 (1970).

[60] D. Henderson, F. F. Abraham, y J. A. Barker, Molecular Physics 31, 1291 (1976).

[61] D. Henderson, en Fundamentals of inhomogeneous fluids, editado por D. Henderson (Marcel Dekker, New York, 1993), Chap. 4. 
[62] R. Kjellander y S. Marcelia, Mol. Phys. 83, 789 (1994).

[6.3] M. Lozada-Cassou, J. Chem. Phys. 75, 1412 (1981).

[64] L. Degrève, M. Lozada-Cassou, E. Sánchez, y E. González-Tovar, J. Chem. Phys. 98, 8905 (1993).

[65] M. Lozada-Cassou, W. Olivares, y B. Sulbarán, Phys. Rev. E 53, 522 (i996).

[66] J. D. Jackson, Classical Electrodynamics (Willey, New York, 1962).

[67] P. Debye y E. Hückel, Phisik. Z. 24, 185 (1923).

[68] J. G. Kirkwood, J. Chem. Phys. 2, 767 (1934).

[69] M. Lozada-Cassou, R. Saavedra-Barrera, y D. Henderson, J. Chem. Phys. 77, 5150 (1982).

[70] M. Metropolis, A. W. Rosenbluth, M.Ñ. Rosenbluth, y E. Teller, J. Chem. Phys. 21, 1087 (1953).

[71] B. J. Alder y T. E. Wainwrigh, J. Chem. Phys. 31, 459 (1959).

[72] D. L. Ermak y J. A. McCammon, J. Chem. Phys. 62, 4189 (1975).

[73] F. de J. Guevara, Comunicación personal.

[74] M. Deserno y C. Holm, J. Chem. Phys. 109, 7678 (1998).

[75] M. Deserno y C. Holm, J. Chem. Phys. 109, 7694 (1998).

[76] M. Deserno, C. Holm, y S. May, Macromolecules 33, 199 (2000).

[77] M. P. Allen y D. J. Tildesley, Computer Simulation of Liquids (Oxford University Press, Oxford, 1987).

[78] M. Born y T. V. Karman, Physik. Z. 13, 297 (1912).

[79] P. Ewald, Ann. Phys(Leipzig) 64, 253 (1921).

[80] M. Lozada-Cassou, J. Phys. Chem. 87, 3279 (1983).

[81] W. Hoppe, Biophysics (Springer Berlag, Berlin, 1983).

[82] G. M. Torrie y J. P. Valleau, J. Chem. Phys. 73, 5807 (1980).

[83] V. Vlachy y A: O. J. Haymet, J. Chem. Phys. 84, 5874 (1986).

[84] L. Degrève, M. Lozada-Cassou, E. Sánchez, y E. González-Tovar, J. Chem. Phys. 98, 8905 (1993). 
[85] L. Degrève y M. Lozada-Cassou, Mol. Phys. 86, 759 (1995).

[86] U. P. Strauss, N. L. Gershfeld, y H. Spiera, J. Am. Chem. Soc. 76, 5909 (1954).

[87] M. Elimelech y C. R. O’Melia, Colloids Surf. 44, 165 (1990).

[88] Y. Wang, K. Kimura, Q. Huang, y P. L. Dubin, Macromolecules 32, 7128 (1999).

[89] Q. R. Huang, P. L. Dubin, C.Ñ. Moorefield, y G. R. Newkome, J. Phys. Chem. B 104, $898(2000)$.

[90] R. Hidalgo-Álvarez y et. al., Adv. Colloid Interface Sci. 67, 1 (1996), artículo de revisión.

[91] M. Lozada-Cassou y D. Henderson, J. Phys. Chem. 87, 2821 (1983).

[92] J. C. Neu, Phys. Rev. Lett. 82, 1072 (1999).

[93] V. Bloomfield, Biopolymers 31, 1471 (1991).

[94] J. P. Valleau, R. Ivkov, y G. M. T. G. M., J. Chem. Phys. 95, 520 (1991).

[95] L. G. Nilsson, L. Guldbrand, y L. Nordenskiöld, Mol. Phys. 72, 177 (1991).

[96] A. P. Lyubartsev, J. X. Tang, P. A. J. L., y Nordenskiöld, Phys. Rev. Lett. 81, 5465 (1998).

[97] M. Lozada-Cassou y D. Henderson, Chem. Phys. Lett. 127, 392 (1986).

[98] R. Kjellander y S. Marcelja, J. Phys. Chem. 90, 1230 (1986).

[99] M. Lozada-Cassou y J. Yu, Phys. Rev. Lett. 77, 4019 (1996).

[100] J. Yu, L. Degrève, y M. Lozada-Cassou, Phys. Rev. Lett. 79, 3656 (1997).

[101] L. Degrève y M. Lozada-Cassou, Phys.Rev. E 57, 2978 (1998).

[102] L. M. y Terán, E. Díaz-Herrera, M. Lozada-Cassou, y R. Saavedra-Barrera, J. Comput. Phys. 84, 326 (1989).

[103] G. Manning, J. Chem. Phys. 51, 924 (1969).

[104] F. D y B. Smit, Understanding Molecular Simulation (Academic Press, San Diego, 1996).

[105] R. W. Hockney y J. W. Eastwood, Computer Simulation Using Particles (IOP, London, 1988).

[106] G. S. Grest y K. Kremer, Phys. Rev. A 33, 3628 (1986). 
[107] R. J. Hunter, Foundations of Colloid Science (Oxford Science Publications, Clarendon Press, Oxford, 1987), Vol. 1.

[108] P. H. Wiersema, A. L. Leob, y J. T. G. Overbeek, J. Colloid Interface Sci. 22, 78 (1966).

[109] R. W. O'Brien y L. R. White, J. Chem. Soc., Farada: Trans. 2 74, 1607 (1978).

[110] B. I. Shlovskii, Phys. Rev. E 64, 041407 (1999).

[111] M. Lozada-Cassou y F. Jiménez-Ángeles, arXiv:physics v2, 0105043 (2001).

[112] G. Decher, Sience 277, 1232 (19) :

[113] J.Ñ. Israelachvili, Accounts Chem. Res. 20, 415 (1987).

[114] F. Mondain-Monval, F. Leal-Calderon, y J: Bibette, J. Phys. II (France) 6, 1313 (1996).

[115] N. Cuviller y F. Rondelez, Thin Solid Films 327-329, 19 (1998).

[116] P. Gonzalez-Mozuelos y J. M. Méndez-Alcaıáz, Phys. Rev. E 63, 21201 (2001).

[117] P. Attard, Advances in Chemical Physics (Wiley, New York, 1966), Vol. XCII.

.118] K. S. Schmitz, Macroions in Solution y Colloidal Suspensions (VCH Publishers, New York, 1993).

[119] D. Grier, Nature 393, 621 (1998).

[120] F. H. Stillinger y R. Lovett, J. Chem. Phys. 48, 3858 (1968).

[121] E. Gurovitch y P. Sens, Phys. Rev. Lett. 82, 339 (1999).

[122] A General Kinetic Theory of Liquids (Cambridge University Press, Cambridge, 1949).

[123] J. Yvon, La Théorie Statistique des Fluides et l'Equation d'Etat, Actualités Scientifiques et Industrielles (Hermann, Paris, 1935), Vol. 203.

- [124] J. G. Kirkwood, J. Chem. Phys. 3, 300 (1935.

[125] D. Henderson, Ann. Rev. Phys. Chem. 15, 31 (i964).

[126] J. K. Percus y G. J. Yevick, Phys. Rev. 110, $1(1958)$.

[127] J. L. Lebowitz y J. Percus, Phys. Rev. 144, $\because 51$ (:966).

[128] J. C. Rasaiah y H. L. Fiedman, J. Chem. Phrs. 59, 3965 (1969). 


\section{BIBLIOGRAFIA}

[129] J. Raisaiah, Chem. Phys. Lett. 7, 260 (1970).

[130] J. C. Rasaiah, J. Chem. Phys. 56, 3071 (1972).

[131] E. Waisman y J. L. Lebowitz, J. Chem. Phys. 56, 3086 (1972).

[132] E. Waisman y J. L. Lebowitz, J. Chem. Phỵs. 56, 3093 (1972).

[133] T. L. Croxton y D. A. McQuarrie, J. Phys. Chem. 83. 1840 (1979).

[134] M. Lozada-Cassou, J. Chem. Phys. 80, 3344 (1984).

[135] G. Arfken, Mathematical Methods for Physicists (Academic Press Inc.. San Diego, CA, 1985).

[136] J. C. Rasaiah, D.N. Card, y J. P. Valleau, J. Chem. Phys. 56, 246 (1972).

[137] M. E. Fisher, J. of Stat. Phys. 75, 1 (1994).

[138] Y. Levine y M. E. Fisher, Physica A 225, 164 (1996).

[139] K. Hiroike, J. Phys. Soc. Japan 27, 1415 (1969).

[140] L. Blum, Mol. Phys. 30, 1529 (1975).

[141] K. Hiroike, Mol. Phys. 33, 1195 (1977).

[142] O. C. Zienkiewicz y R. L. Taylor, The Finite Element Method, 4th ed. (McGraw Hill Book Company, London, 1989), Vol. 1. 